\title{
Modenstruktur und adaptive Regelung der Strahl-Kanten-Strömung
}

\author{
Dissertation \\ zur Erlangung des Doktorgrades \\ der Mathematisch-Naturwissenschaftlichen Fakultäten \\ der Georg-August-Universität zu Göttingen
}

vorgelegt von

\author{
Arno Ickler \\ aus
}

Bad Hersfeld

Göttingen 2004 


\section{D7}

Referent:

Korreferent:
Prof. Dr. D. Ronneberger

Prof. Dr. U. Parlitz

Tag der mündlichen Prüfung: 30.11.2004 


\section{Inhaltsverzeichnis}

1 Einleitung 1

2 Experimenteller Aufbau des Strahl-Kanten-Systems 5

2.1 Der Strömungskanal . . . . . . . . . . . . . . . . . . . . . 5

2.2 Regelkreis . . . . . . . . . . . . . . . . . . . . . 8

2.3 Optimale Interpolation der Streichlinienposition . . . . . . . . . . . . . . . 9

2.4 Hysterese der Aktuatoranregung . . . . . . . . . . . . . . . . . . . . . 15

3 Modellierung der konvektiven Instabilität 21

3.1 Rayleigh-Gleichung . . . . . . . . . . . . . . . . . . . . . . . . . 21

3.2 WKB-Methode für nahezu parallele Strömungen . . . . . . . . . . . . . . . . . . 23

3.3 Parametrisierung des Gleichströmungsprofils . . . . . . . . . . . . . . . . . . 24

3.4 Anfachung und Phase des instationären Strömungsfelds und der Streichlinien . . 27

3.5 Nichtlinearität der Freistrahlinstabilität bezogen auf die Streichlinienauslenkung 31

3.5.1 Sättigungsverhalten der Freistrahlinstabilität . . . . . . . . . . . . 32

3.5.2 Nichtlinearität der Streichlinienintegration . . . . . . . . . . . . 33

3.6 Amplitudenabhängigkeit des Phasengradienten . . . . . . . . . . . . . . . 34

3.7 Modellierung der Freistrahlinstabilität durch eine Übertragungskette . . . . . . . 36

3.7.1 Bestimmung der Impulsantworten der Übertragungskettenglieder . . . . . 37

3.7.2 Modellierung der Nichtlinearität . . . . . . . . . . . . . . 41

3.8 Modellierung der globalen Instabilität durch digitale Filter . . . . . . . . . . . . 42

4 Struktur und Dynamik der Moden $\quad 45$

4.1 Zerlegung der Strahl-Kanten-Strömung in konzentrierte Elemente . . . . . . . . 46

4.2 Messverfahren . . . . . . . . . . . . . . . . . . . . . . . . 48

4.3 Räumliche Struktur: Phasenbeziehungen und Wellenzahl . . . . . . . . . . . . . 49

4.3.1 Phasenbeziehung zwischen den Streichlinienauslenkungen an Düse und Keil 50

4.3.2 Phasendrehung bei der Instabilitätswellenausbreitung . . . . . . . . . . 53 
4.4 Zusammenhang zwischen räumlicher und zeitlicher Struktur: Modenspektrum . . 54

4.4.1 Absinken der Strouhalzahl mit dem Düse-Keil-Abstand . . . . . . . . . . 55

4.4.2 Anstieg der Strouhalzahl mit der Reynoldszahl . . . . . . . . . . . . . 56

4.5 Räumliche Amplitudenentwicklung der Moden . . . . . . . . . . . . . . . . . 57

4.6 Hysterese und Systemzustände aus mehreren Moden . . . . . . . . . . . . . . . 61

4.7 Dynamik der Moden . . . . . . . . . . . . . . . . . . . . 66

4.7.1 Synchronisation der Moden . . . . . . . . . . . . . . . 67

4.7.2 Tieffrequente Dynamik der Moden . . . . . . . . . . . . . . . 69

4.8 Erzwungene Modenübergänge . . . . . . . . . . . . . . . . . . . . 82

5 Regelung des Strahl-Kanten-Systems $\quad 85$

5.1 Phasenverschiebungsregler . . . . . . . . . . . . . . . . . . 87

5.2 Modell einer frequenzunabhängigen Keilübertragungsfunktion ...... . 90

5.3 Adaptive Regelung . . . . . . . . . . . . . . . . . . . . . . . 94

5.3.1 Entwurf des adaptiven Regelalgorithmus . . . . . . . . . . . . . 95

5.3.2 Messung der Übertragungsfunktionen und Gegenkopplungsberechnung 96

5.3.3 Stabilität des adaptiven Algorithmus und Wahl der Zielfunktion . . . . . 98

5.4 Parameter und Grenze der adaptiven Regelung . . . . . . . . . . . . . . . . . . 103

5.4.1 Wahl der optimalen Regelposition . . . . . . . . . . . . . . . 104

5.4.2 Parallel laufende adaptive Algorithmen . . . . . . . . . . . . . . . 105

5.4.3 Grenze der Regelung in der Re-L/d-Ebene . . . . . . . . . . . . . . 106

5.5 Übertragungsfunktion der Strahl-Kanten-Wechselwirkung . . . . . . . . . . . . . 110

6 Zusammenfassung und Ausblick 115

A Anhang A: Stabilität der Lösung der Rayleighgleichung gegenüber kleinen $\begin{array}{lr}\text { Profilstörungen } & 119\end{array}$

A.1 Stabilität der Lösung der Rayleighgleichung gegenüber kleinen Profilstörungen 119

B Anhang B: Nichtlinearität $\quad 121$

B.1 Streichlinien und Sättigungsverhalten der Strahlinstabilität . . . . . . . . . . . . 121

B.1.1 Verbreiterung durch die Rückwirkung der Wechselschubspannungen auf das Gleichströmungsprofil . . . . . . . . . . . . . . . . . . . . . 121

B.1.2 Verbreiterung des Gleichströmungsprofils durch die Mittelung des instationär verschobenen Gleichströmungsprofils . . . . . . . . . . . . . . . . . 124

B.1.3 Streichlinienintegration bei Überlagerung zweier verschieden frequenter Geschwindigkeitsfelder . . . . . . . . . . . . . . 125

B.1.4 Höher-harmonische Spektralkomponenten als Maß für die Nichtlinearität 126 
B.1.5 Streichlinienauslenkung in Abhängigkeit von der mittleren Position der Streichlinie . . . . . . . . . . . . . . . . 127

C Anhang C: Rezeptivität

C.1 Normierung der Streichlinienauslenkung auf die Auslenkung des Potenzialfeldes . 132

C.2 Strahlrezeptivität . . . . . . . . . . . . . . . . . . 132

C.2.1 Zum Einfluss der Strömung auf die laterale Geschwindigkeit der Teilchen 134

C.3 Anregung der Instabilitätswelle . . . . . . . . . . . . . . . . . . . 138

C.4 Bereinigung der Streichlinienauslenkung . . . . . . . . . . . . . . . 139

D Anhang D: Modell der konvektiven Instabilität 143

D.1 Modellierung der konvektiven Instabilität . . . . . . . . . . . . . . . . . 143

D.1.1 Begrenzung des räumlichen Differenzsignals . . . . . . . . . . . . . 143

D.1.2 Begrenzung der zeitlichen Ableitung des Signals . . . . . . . . . . . . . . 144

D.1.3 Begrenzung des Signals . . . . . . . . . . . . . . . . . . 145

$\begin{array}{ll}\text { E Anhang E: Modell des Strahl-Kanten-Systems } & 147\end{array}$

E.1 Modell des Strahl-Kanten-Systems auf die Streichlinienauslenkug bezogen . . . . 147

F Anhang F: Karhunen-Loève-Transformation 151

F.1 Analytisches Signal durch Karhunen-Loève-Transformation . . . . . . . . . . . . 151

Literaturverzeichnis

155 



\section{Bezeichnungen}

\section{Konventionen und Operatoren}

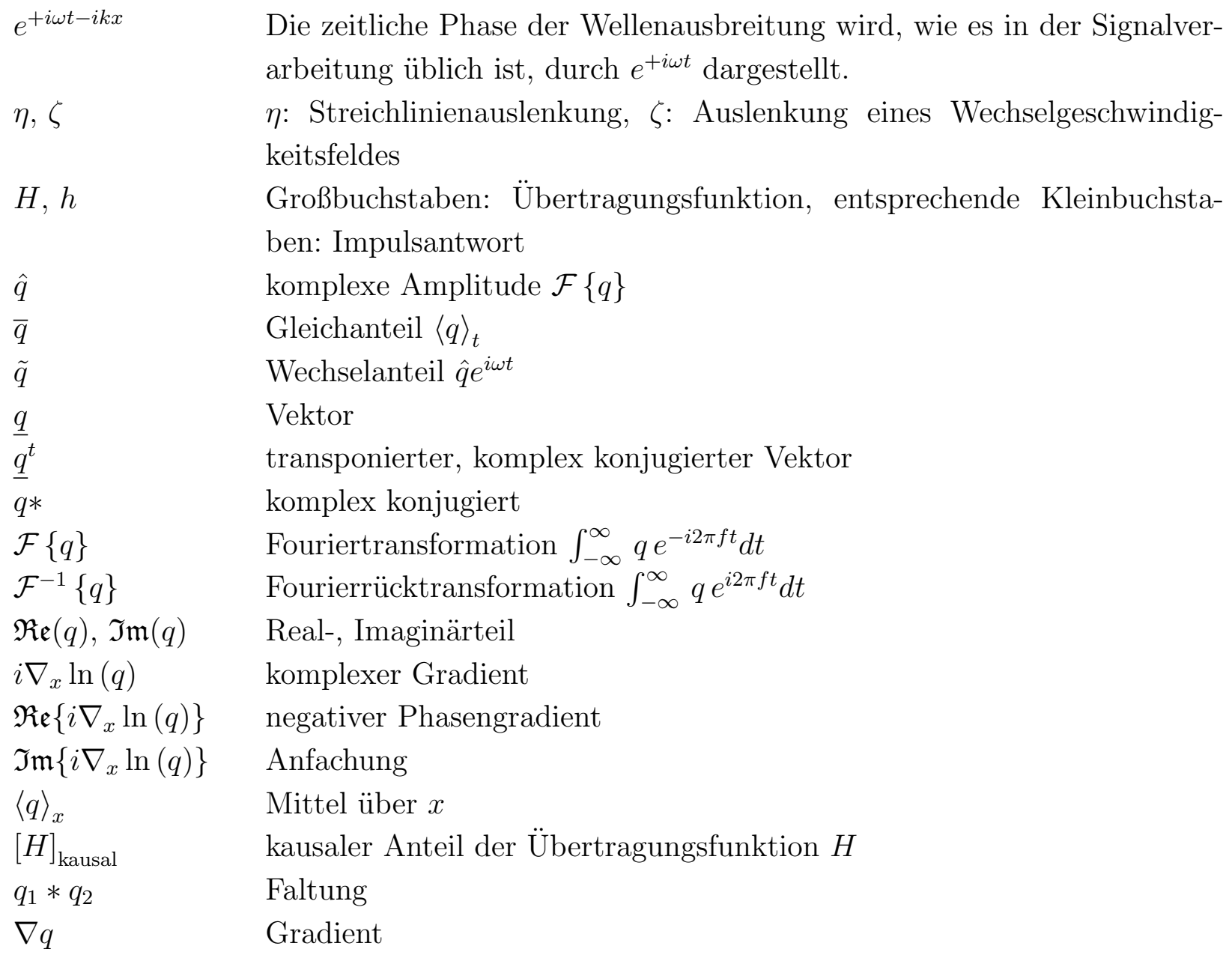

\section{Abkürzungen}

psd power spectral density, spektrale Leistungsdichte

krit. kritisch 


$\begin{array}{ll}\text { Mess. } & \text { Messung } \\ \text { Reg. } & \text { Regelung } \\ \text { Akt. } & \text { Aktuator } \\ \text { Inst. } & \text { Instabilitätswelle } \\ \text { WKB } & \text { Wentzel-Kramers-Brillouin-Jeffreys-Methode } \\ \text { Ray } & \text { Rayleigh-Gleichung } \\ \text { SKWW } & \text { Strahl-Kanten-Wechselwirkung } \\ P D F & \text { probability density function, Häufigkeitsdichte } \\ \text { opt } & \text { optimal } \\ \text { rms } & \text { root mean square, } \sqrt{\left\langle q^{2}\right\rangle_{t}}\end{array}$

\section{Symbolverzeichnis}

$\hat{a}_{\text {Akt. }} \quad$ Aktuatorauslenkungsamplitude in Steps (Definiton siehe Anhang C.1)

$\hat{a}_{\mathrm{Akt}}^{\mathrm{ae}}$ äquivalente Aktuatorauslenkung in Steps: wird gebraucht, um die gleiche Wirkung, wie die der Strahl-Kanten-Wechselwirkung hinsichtlich der Anregung der Instabilitätswelle, zu erzielen

A

$A_{\text {Inst }}$

$A^{\text {asymp. }}$

$\hat{a}_{\mathrm{Eff}}$.

$\hat{a}_{\text {Hyst. }}$

$A_{0}$

$A_{\mathrm{WKB}}$

a

$b$

$B$

$d$

$d_{\text {Strahl }}$

$d_{\text {Strahl,end }}$

$\underline{E}$

$\frac{E_{\mathcal{C}}}{\underline{E}^{\mathrm{TP}}}$
Aktuatorübertragungsfunktion $\hat{\eta}\left(x_{\mathrm{D}}\right) / \hat{a}_{\mathrm{Akt}}$.

Aktuatorübertragungsfunktion $\hat{\eta}_{\text {Inst. }}\left(x_{\mathrm{D}}\right) / \hat{a}_{\text {Akt. }}$. bezogen auf den Instabilitätswellenanteil der Streichlinienauslenkung am Ort $x_{\mathrm{D}}$ asymptotische Aktuatorübertragungsfunktion für große Amplituden effektiver Anteil bzw. Wirkanteil der Aktuatoramplitude $\hat{a}_{\text {Akt. }}$ Hysterese Anteil der Aktuatoramplitude $\hat{a}_{\text {Akt. }}$ Übersetzungsfaktor der Instabilitätswelle der WKB-Lösung auf das tatsächliche Instabilitätswellenwechselfeld normierte Korrekturfunktion der WKB-Methode

Parameter des Hauptsrömungsprofil (Gl. 3.7)

Parameter des Hauptsrömungsprofil (Gl. 3.7)

Kanaltiefe ( $z$-Richtung): $40 \mathrm{~mm}$

Düsenbreite

Maß für die Strahlbreite

instationär erreichte Breite des Strahlprofils am Keil räumlicher Eigenvektor der auf den rms-Wert normierten Streichlinienbewegung

räumlicher komplexer Eigenvektor, der aus den $\underline{E}$ zusammengesetzt wird räumlicher Eigenvektor des tieffrequenten Anteils der Zeitreihe 
$\underline{E}^{\mathrm{HP}}$

$f_{\mathrm{s}}$

$f_{\mathrm{m}}$

$f_{\text {sampling }}$

$\tilde{f}_{\text {SKWW }}\left(x_{\mathrm{D}}\right)$

F

$f^{\mathrm{H}}$

$f^{\mathrm{T}}$

$f_{\mathrm{I}}$

$H_{-1}, H_{0}, H_{1}$

$H_{\text {dq }}$

$H_{\text {soll }}$

$H^{(g)}$

i

$J$

$k$

K

$K_{\text {ww }}$

$K_{\mathrm{L}}$

L

$L_{\text {krit. }}$

$\mathrm{n}$

$n_{0}$

p

Pixel

$\nabla \tilde{p}_{\text {SKWW }}$

$\nabla \tilde{p}_{\text {Akt. }}$

$p_{\mathcal{C}}$

$p_{\mathcal{C}}^{\mathrm{TP}}$

$p_{\text {Transfer }}$

$P_{\text {in }}$

$\mathrm{Re}_{\mathrm{d}}$ räumlicher Eigenvektor des hochfrequenten Anteils der Zeitreihe Störfrequenz

Frequenz der Mode $m$

Samplingfrequenz $50 \mathrm{~Hz}$

durch die Strahl-Kanten-Wechselwirkung induzierte Kraftdichte am Ort $x_{\mathrm{D}}$

Feedback, physikalische Rückkopplung

Frequenz der Mode II in Systemzustand II

Frequenz der Mode I in Systemzustand II

Frequenz der Mode I in Systemzustand I

Helligkeit am Ort $n_{0}-1, n_{0}, n_{0}+1$

Quotient der Differenzen der Helligkeitswerte

Sollübertragungsfunktion von $\hat{\eta}(x) / \hat{a}_{\text {Akt. }}$.

Übertragungsfunktion des geschlossenen Regelkreises

imaginäre Einheit $\sqrt{-1}$

Impulsfluss

komplexe Wellenzahl aus der Dispersionsrelation

Verstärkungsfaktor

Übertragungsfunktion der SKWW

Übersetztungsfaktor für die Aktuatorübertragungsfunktion bei verschiedenen Keilabständen

Düse-Keil-Abstand, $L=x_{\text {Keil }}-x_{\mathrm{D}}$

kritischer Düse-Keil-Abstand, für $L>L_{\text {krit. versagt die Regelung }}$

Pixelnummer

Pixelnummer mit minimaler Helligkeit

Druck

Bildelement des CCD-Chips

Wechseldruckfeld der Strahl-Kanten-Wechselwirkung als Funktion von $x$, y

Wechseldruckfeld des Aktuators als Funktion von $x, y$

komplexer Projetionskoeffizient der Karhunen-Loève-Transformation

Projetionskoeffizient der Karhunen-Loève-Transformation

Leistungstransferfaktor

Leistung der Streichlinienauslenkung an einer bestimmten $x$-Position

Reynoldszahl $\frac{U_{\mathrm{D}} d}{\nu}$ bezogen auf die Düsenbreite $d$ 
VIII

\begin{tabular}{|c|c|}
\hline $\mathrm{Sr}_{\mathrm{d}}$ & Strouhalzahl $\frac{\omega d}{U_{\mathrm{D}}}$ bezogen auf die Düsenbreite $d$ \\
\hline $\mathrm{Sr}_{\mathrm{end}}$ & am Keil erreichte Strouhalzahl $\frac{\omega d_{\text {Strahl,end }}}{U_{\mathrm{D}}}$ \\
\hline $\mathrm{Sr}_{\mathrm{L}}$ & Strouhalzahl $\frac{\omega L}{U_{\mathrm{D}}}$ bezogen auf den Düse-Keil-Abstand $L$ \\
\hline Step & $\begin{array}{l}\text { 1/2000 Grad Drehung der Gewindestange der Kolbenpumpe durch den } \\
\text { Schrittmotor }\end{array}$ \\
\hline$\Delta S$ & Strahlübertragungsfunktion $\hat{\eta}(x+\Delta x, \omega) / \hat{\eta}(x, \omega)$ \\
\hline$S_{1}$ & Strahlübertragungsfunktionen $\hat{\eta}\left(x_{\text {Regel }}\right) / \hat{\eta}\left(x_{\mathrm{D}}\right)$ \\
\hline$S_{2}$ & Strahlübertragungsfunktionen $\hat{\eta}\left(x_{\text {Keil }}\right) / \hat{\eta}\left(x_{\text {Regel }}\right)$ \\
\hline$\Delta s$ & Impulsantwort $\mathcal{F}^{-1}\{\Delta S\}$ \\
\hline$s(t)$ & Eingangssignal für Aktuator (ohne Gegenkopplung) \\
\hline$\left|s^{\max }\right|$ & maximale Amplitude des Eingangssignals \\
\hline$\left|s^{\max }\right|_{\text {Start }}$ & maximale Amplitude des Eingangssignals zu Beginn der Messung \\
\hline$\left|s^{\max }\right|_{\text {Ende }}$ & maximale Amplitude des Eingangssignals am Ende der Messung \\
\hline$T_{0}$ & Temperatur des Wassers im Kanal \\
\hline$\Delta T$ & Temperaturdifferenz zwischen Strahl und Wasser im Kanal \\
\hline$u, U$ & Geschwindigkeitskomponente in Hauptsrömungsrichtung \\
\hline$U_{\mathrm{D}}$ & Durchflussgeschwindigkeit Volumen/Zeit \\
\hline$U_{0}$ & Maximum von $\bar{U}(y)$ über alle $y$ und Profilparameter (Gl. (3.7)) \\
\hline$U_{\text {ausgl. }}$ & Geschwindigkeit der Ausgleichsströmung in Strahlnähe (Gl. (3.7)) \\
\hline $\bar{U}^{(\mathrm{n})}$ & $x$-Komponente des Hauptsrömungsprofils im $n$-ten Iterationsschritt \\
\hline $\bar{U}_{\text {Inst. }}$ & $x$-Komponente des verbreiterten Hauptströmungsprofils \\
\hline$v, V$ & Geschwindigkeitskomponente senkrecht zur Hauptströmungsrichtung \\
\hline$\dot{V}$ & Volumenfluss \\
\hline$v_{\text {Akt. }}^{\mathrm{n}}$ & Normierungskonstante der Wechselgeschwindigkeit (Anhang C.1) \\
\hline$\tilde{v}_{\text {Inst }}^{\text {eff. }}$ & $\begin{array}{l}\text { Wechselgeschwindigkeit am Ort des effektiven Wechselwirkungszentrums } \\
\text { der SKWW }\end{array}$ \\
\hline$v^{\mathrm{H} / \mathrm{T}}$ & Frequenzverhältnis des höherfrequenten zu dem niederfrequenten Peak \\
\hline$x$ & Ortskoordinate in Hauptströmungsrichtung \\
\hline$x_{\mathrm{D}}$ & $x$-Position der Düse \\
\hline$x_{\text {Keil }}$ & $x$-Position des Keils \\
\hline$x_{\text {start }}$ & $x$-Koordinate des Farbstoff-Injektionspunktes \\
\hline$x_{\text {mess }}$ & $x$-Position, an der die Streichlinienauslenkung gemessen wird \\
\hline$x_{\text {Regel }}$ & $\begin{array}{l}x \text {-Position, an der die Streichlinienauslenkung gemessen wird, die zur } \\
\text { Regelung verwendet wird }\end{array}$ \\
\hline
\end{tabular}




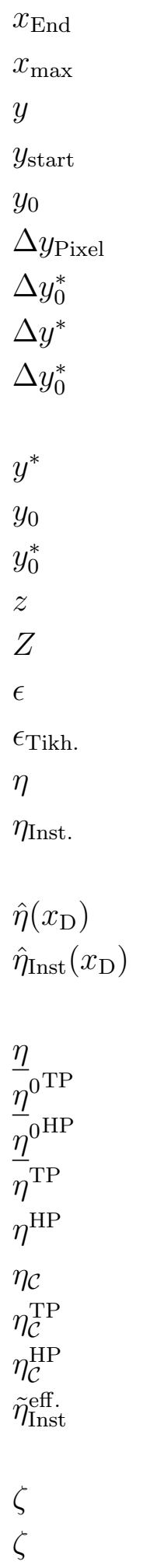

$x$-Position des letzten Filters der Filterkaskade

$x$-Position des Maximums des Aktuatorpotenzialfeldes für $y \equiv 0$

Ortskoordinate senkrecht zur Hauptströmungsrichtung

$y$-Koordinate des Farbstoff-Injektionspunkt

laterale Integrationsgrenze für Volumen- und Impulsfluss.

Breite eines Bildelements

normierte Position des interpolierten Minimums relativ zu $n_{0}$

auf $\Delta y_{\text {Pixel }}$ normierte Position innerhalb eines Pixels

auf $\Delta y_{\text {Pixel }}$ normierte relative Position des interpolierten Helligkeitsmini-

mums innerhalb eines Pixels

auf $\Delta y_{\text {Pixel }}$ normierte Position der Helligkeitswerte

Position des interpolierten Helligkeitsminimums innerhalb eines Pixels

normierte Position des interpolierten Minimums

Ortskoordinate in spannweitiger Richtung (Kanaltiefe)

Zielfunktion des adaptiven Algorithmus

relative Änderung der Gleichströmung mit dem Laufweg (Gl. (3.5))

Tikhonov-Regularisierungskonstante

Streichlinienauslenkung

Streichlinienauslenkung auf Grund des Wechselgeschwindigkeitsfelds der Instabilitätswelle

Streichlinienamplitude unterhalb der Düse

Streichlinienamplitude unterhalb der Düse aufgrund des Wechselgeschwindigkeitsfelds der Instabilitätswelle

Spaltenvektor aller Messpositionen der Streichlinienauslenkung

tieffrequenter Anteil von $\eta^{0}$

hochfrequenter Anteil von $\underline{\eta}^{0}$

tieffrequenter Anteil der Zeitreihe $\eta$

hochfrequenter Anteil der Zeitreihe $\eta$

analytisches Signal von $\eta$

analytisches Signal von $\eta^{\mathrm{TP}}$

analytisches Signal von $\eta^{\mathrm{HP}}$

Streichlinienauslenkung am Ort des effektiven Wechselwirkungszentrums der SKWW

Auslenkung des Potenzialfeldes

Auslenkung eines Wechselgeschwindigkeitsfeldes 
$\mathrm{X}$

$\zeta_{\text {Akt. }} \quad$ Auslenkung des Potenzialfeldes des Aktuators für $y \equiv 0$

$\zeta_{\text {Akt. }}^{\mathrm{n}}$

Normierungskonstante der Streichlinienauslenkung (Anhang C.1)

$\nu$

dynamische Viskosität

$\tau_{\nu}$

Schubspannung auf Grund der Viskosität

$\bar{\tau}_{\text {Inst. }}$

$\bar{\tau}_{\text {Inst. }}^{\text {(n) }}$

Schubspannung auf Grund der Instabilitätswelle

Schubspannung auf Grund der Instabilitätswelle im $n$-ten Iterationsschritt

$\Phi$

Stromfunktion

$\tilde{\Phi}_{\mathrm{WKB}}$

$\varphi_{\eta}$

$\Delta \Phi^{\mathrm{TP}}$

$\Delta \Phi^{\mathrm{HP}}$

$\Delta \Phi^{\mathrm{TPTP}}$

$\Delta \Phi^{\mathrm{HPTP}}$

Stromfunktion der WKB-Lösung

Phase der Streichlinienauslenkung einer bestimmten Frequenz

Schwankungen der tieffrequenten Phase um die lineare Phase $f^{\mathrm{T}} \cdot t$

Schwankungen der hochfrequenten Phase um die lineare Phase $f^{\mathrm{H}} \cdot t$

$\omega$

$\omega_{\mathrm{s}}$

$\omega_{\text {grenz }}$

$\omega_{\mathrm{s}}^{\prime}$

$\omega_{\mathrm{i}}$

$\omega_{\mathrm{m}}$

$\Delta \Phi^{\mathrm{TP}}$ mit Tiefpass gefiltert

$\Delta \Phi^{\mathrm{HP}}$ mit Tiefpass gefiltert

Kreisfrequenz

Störkreisfrequenz $2 \pi f_{\mathrm{s}}$

Grenzfrequenz

Dopplerverschobene Frequenz

Kreisfrequenz des Spitzenwertes des Leistungsspektrums der Moden die Mode $m$ kennzeichnende Kreisfrequenz 


\section{Kapitel 1}

\section{Einleitung}

Die Strahl-Kanten-Strömung ist eine der einfachsten selbsterregt schwingenden Strömungssysteme: Ein aus einer Düse austretender ebener laminarer Freistrahl trifft auf eine Kante. Kleine Störungen des Freistrahls werden durch die Freistrahlinstabilität verstärkt und wachsen stromab mit dem Laufweg an. Trifft der Strahl auf die Kante, so wird durch die Strahl-KantenWechselwirkung ein Druckfeld erzeugt, das wiederum eine Störung auf den Freistrahl zurückkoppelt. Diese Rückkopplung verursacht die selbsterregte Schwingung der Strahl-Kanten-Strömung (siehe z. B. Powell 1961 [38]).

Die Strahl-Kanten-Strömung ist eine sehr effektive Schallquelle. In Verbindung mit einem Resonator, wird die Strömung zur Tonerzeugung in Flöten und Orgelpfeifen genutzt. Beim sogenannten „Cabin Boom“ oder Wummern (geöffnetes Fahrzeugfenster bei größerer Geschwindigkeit) soll dieser Schall unterdrückt werden, was meistens durch Veränderung der Anströmung (passive Strömungskontrolle) erreicht wird.

In dieser Arbeit soll aktiv durch eine Regelung mittels einer Druckanregung die Strahl-KantenStrömung beeinflusst werden, so dass die dem System innewohnende Instabilität verschwindet. Durch diese Stabilisierung wird der durch die Grenzzyklus-Oszillationen verdeckte Blick auf die Dynamik des Systems frei. Damit kann das System in einzelne Elemente zerlegt werden, die nun

im Rahmen des Gesamtsystems untersucht werden können. Dies führt zu einem tiefergehenden Verständis der Prozesse, die am Grenzzyklus beteiligt sind.

Zur gezielten Beeinflussung der Systemdynamik wird ein Regelsystem benutzt, das auf einer Echtzeit-Videobildanalyse basiert. Die Strömung wird durch Einfärben einer Streichlinie sichtbar gemacht. Mit CCD-Kamera und Computer wird die raum-zeitliche Bewegung der Streichlinie aufgenommen und ausgewertet. Aus diesen Informationen lässt sich mit geeigneten Algorithmen ein zweckmäßiges Regelsignal errechnen.

Es besteht auch die Möglichkeit, steuernd in die Systemdynamik einzugreifen, indem man geeignete Signale einstreut. Durch die Steuerung kann das System beispielsweise zu einem definierten Zustandswechsel bewegt werden. Eine Unterdrückung der Instabilität ist damit jedoch nicht möglich. 
In dieser Arbeit soll die Grenze der Regelbarkeit dieses an sich instabilen Strömungssystems aufgezeigt und geeignete Regelalgorithmen entwickelt werden. Dabei ist von besonderem Interesse, welche Größen man beobachten muss, um das System optimal regeln zu können und auf welchen Zeitskalen sich die für die Regelung relevante Dynamik abspielt. Dazu ist auch eine Untersuchung der Dynamik des im Grenzzyklus schwingenden Systems nützlich, die in dieser Arbeit vorgenommen wird.

\section{Modenstruktur und Dynamik der Moden}

Das Modenspektrum der Strahl-Kanten-Strömung entsteht im wesentlichen durch die Ausbreitung der Strahlinstabilität kombiniert mit der Strahl-Kanten-Wechselwirkung. Von Interesse ist dabei, wie die Frequenz der selbsterregten Schwingung mit den Strömungsparametern skaliert. Experimentell hat als erster Sondhaus 1854 [43] das Modenspektrum untersucht, weitere experimentelle Untersuchungen stammen von Brown 1937 [5], NyBOrg 1952 [30], Brackenridge 1960 [4] und Bamberger 2004 [1]. Theoretische Modelle der Strahl-KantenWechselwirkung und der Anregung der Instabilitätswelle (Powell 1961[38], Holger et al. 1977 [19], CRighton 1992 [8]) können anhand des vorausgesagten Modenspektrums und der Modenstruktur im Vergleich zu experimentellen Daten auf ihre Güte hin überprüft werden.

In dieser Arbeit soll durch das hochpräzise Messsystem eine genaue Erfassung des Modenspektrums und der Modenstruktur vorgenommen weden. Um eine Interpretation der Ergebnisse zu ermöglichen wird die Amplitudenabhängigkeit der konvektiven Strahl-Instabilität modelliert und das Messverfahren, das auf der Auswertung einer Streichlinie basiert, untersucht.

Die Systemzustände der Strahl-Kanten-Strömung bestehen nicht unbedingt aus einer monofrequenten Schwingung, es werden z. B. auch Subharmonische der Strahlschwingung beobachtet (LuCAS \& Rockwell 1984 [26]). Die Dynamik der Systemzustände mit mehreren Moden wird hier untersucht. Die zunächst stationär stochastisch erscheinende Dynamik der Moden entpuppt sich bei genauerer Betrachtung als sehr autonom und wenig stochastisch. Die Systemzustände, in denen mehrere Moden koexistieren, zeigen Modensynchronisation auf langen Zeitskalen bezüglich der Schwingungsdauer. Die Dynamik dieser Moden ist insbesondere für die Regelung von Interesse, da die Wechselwirkung der Moden, die für die Synchronisation verantwortlich ist, ähnlich einer Regelung fungiert und daher mit der Grenze der Regelbarkeit in Verbindung stehen kann.

\section{Adaptive Regelung}

Die Grenze der Regelung hängt für das Strahl-Kanten-System und dem hier verwendeten Messund Regelsystem von den verwendeten Regelalgorithmen ab. Trotz dieser speziellen Wahl lassen sich Eigenschaften dieses Systems bezüglich einer Regelung aufzeigen und auch verallgemeinern, die beim Entwurf von Reglern zu beachten sind und die die Grenze der Regelbarkeit festlegen. 
Die Strahl-Kanten-Strömung ist besonders geeignet, um mit wenigen Parametern (Durchflussgeschwindigkeit $U_{\mathrm{D}}$, Kantenabstand $L$, Düsenbreite $d$ ) sehr unterschiedlich schwere Regelungsaufgaben zu erzeugen. Eine Stabilisierung des Systems verlangt mit steigender Anzahl leicht zu aktivierender Freiheitsgrade eine zunehmend genauere Systemidentifikation und immer ausgefeiltere Regelalgorithmen.

Der Entwurf derartiger Regelalgorithmen benötigt eine genaue Modellierung der Dynamik der Strahl-Kanten-Strömung, die auf Grund der folgenden Systemeigenschaften erschwert wird.

Verteilung: Das System hat im Gegensatz zu den meisten elektrischen und mechanischen Systemen eine sehr hohe Anzahl von Freiheitsgraden, die auch sehr leicht aktiviert werden können.

Nichtlinearität: Die Strahlinstabilität sättigt in einer schwer beschreibaren Weise. Dabei ist unter anderem auch die Ausbreitungsgeschwindigkeit der Instabilitätswelle amplitudenabhängig. Die amplitudenabhängige Modellierung der Strahl-Kanten-Wechselwirkung ist ein offenes Problem. Es steht noch kein konzentriertes Element im Sinne einer Übertragungsfunktion der Strahlauslenkung auf die Dipolstärke und eines nichtlinearen Gliedes zur Verfügung. Eine Identifizierung der Übertragungscharakteristik ist im Rahmen dieser Arbeit mittels adaptiver Regelung möglich.

Unbekannte offene Systemdynamik: Für die Modellierung des Systems und den damit möglichen Entwurf von Reglern ist die Kenntnis der offenen (open-loop) Systemdynamik notwendig, die auf Grund der physikalisch geschlossenen Rückkopplungsschleife nicht ermittelt werden kann. Die einzelnen Übertragungscharakteristika des offenen Systems lassen sich daher nur näherungsweise aus der Systemdynamik ohne Keil ableiten, da sich durch das Entfernen des Keils die Dynamik der Strahlinstabilität und der Anregung ändert.

Die thermoakustische Schwingung der Luftsäule in einem erhitzten Rohr (sogenanntes RijkeRohr) ist ebenfalls ein intrinsisch rückgekoppeltes, instabiles, verteiltes System, dessen offene Systemdynamik unbekannt ist (VAUdREY 2001 [44]). Die dort verwendeten Regel-Methoden sind auf die Strahl-Kanten-Strömung übertragbar.

Aufgrund der unbekannten offenen Systemdynamik müssen im wesentlichen modellfreie Ansätze zur Regelung herangezogen werden.

Als einfachste Methode zur Stabilisierung selbsterregt schwingender Systeme kann eine Messgröße verstärkt und phasenverschoben gegengekoppelt werden. Dieser Reglertyp kann verschiedene Systeme bis zu einem gewissen Grad stabilisieren: „compressor surge“ [13], überströmte Hohlräume [29] und Kàrmànsche Wirbelstraße [42]. Dieser Regler wurde für das Strahl-KantenSystem von Preckel 1999 [39] und Fujisawa et al. 2004 [14] [15] untersucht, wobei die Stabilisierung auf diese Art nur für relativ kleine Düse-Keil-Abstände möglich ist. Ebenso sind 
genetische Algorithmen - durch geschicktes Kombinieren (lineares Superponieren) von Gegenkopplungen wird die am besten stabilisierende ausgewählt - nur für kleine Düse-Keil-Abstände erfolgreich (PRECKEL).

Für die thermoakustische Instabilität werden auch LMS-Algorithmus Varianten (Least Mean Square), wie der sogenannte filtered-u oder der filtered-e Algorithmus, erfolgreich angewendet, wobei der Fehlersensor und das Referenzsignal durch die Rückkopplung des Systems an der gleichen Stelle entnommen werden (Kuo \& Morgan 1996 [31], Billoud et al. 1992 [3]). Der filtered-u LMS wurde am Strahl-Kanten-System ohne Erfolg angewendet. Zunächst wird zwar für kleine Düse-Keil-Abstände eine Stabilisierung erreicht, allerdings konvergiert das Gegenkopplungsfilter nicht, und letztendlich wird das Gesamtsystem instabil (PRECKEL). Aus der Nichtlinearen Dynamik stammende Regelungsmethoden, wie von Oтt, Grebogi \& YORK 1990 [35] zur Stabilisierungen instabiler Orbits, können auf den hier betrachteten Parameterbereich des Strahl-Kanten-Systems nur bedingt angewendet werden, da kein chaotisches Verhalten beobachtet wird.

In dieser Arbeit wird unter anderem ein adaptiver Algorithmus entwickelt, der auf linearer Systemtheorie basiert und in der Lage ist, die veränderte Systemdynamik des im Grenzzyklus schwingenden Systems stückweise linear zu durchfahren. Diese adaptive Regelung passt eine Gegenkopplung derart an, dass die Systemdynamik des Strahl-Kanten-Systems sich der Systemdynamik der konvektiven Instabilität annähert. Dabei analysiert der adaptive Algorithmus das im Grenzzyklus schwingende System, verändert mit dieser Information die Gegenkopplung und stabilisiert so die Dynamik des Systems. 


\section{Kapitel 2}

\section{Experimenteller Aufbau des Strahl-Kanten-Systems}

Im Folgenden wird der Aufbau des Strömungskanals und des Regelsystems erläutert. Der Strömungszustand der Strahl-Kanten-Strömung wird durch eine im Strahl angefärbte Streichlinie charakterisiert. Die dadurch gewonnene räumliche und zeitliche Information über die Strömung kann genutzt werden, um eine Druckfeldaktuator anzusteuern. Die durch die Streichlinie gewonnene Information lässt aber nur indirekt auf das Strömungsfeld schließen (siehe 3.5), im Gegensatz zu Verfahren wie Particle Image Velocimetry (PIV), Laser Doppler Anemometrie (LDA) oder Doppler Global Velocimetry (DGV). Jedoch ist das hier verwendete Verfahren einfacher aufzubauen. Der experimentelle Aufbau des Strahl-Kanten-Systems, des Bildaufnahmeund Bildauswertesystems, sowie der Aktuatoren sind im Detail in der Dissertation von PRECKEL 1999 [39] zu finden. Im Folgenden wird nur dort detailliert auf das System eingegangen, wo gegenüber PRECKEL Verbesserungen erreicht wurden.

\subsection{Der Strömungskanal}

Der vertikal stehende Wasserkanal wird durch die Schwerkraft getrieben, wobei der Wasserpegel durch ein Überlaufbecken konstant gehalten wird (siehe Abb. 2.1). Damit erreicht man eine sehr konstante Strömungsgeschwindigkeit. Diese kann durch ein Nadelventil eingestellt werden, und sie wird mittels eines Durchflussmessers auf circa $0.2 \%$ genau gemessen. Der Volumenfluss kann maximal 0.7 l/min betragen, das entspricht je nach Düse einer maximalen Strömungsgeschwindigkeit $U_{\mathrm{D}}$, die im folgenden Durchflussgeschwindigkeit genannt wird, von $80-160 \mathrm{~mm} / \mathrm{s}$. Die Wassertemperatur erhöht sich bei längerer Betriebsdauer durch die Wärmeabgabe der Pum-

pen und stellt sich unter Normalbedingungen (Labortemperatur $22^{\circ} \mathrm{C}$ ) bei $27^{\circ} \mathrm{C}$ ein. Diese Temperaturerhöhung führt bei einer fest eingestellten Nadelventilstellung zu einer Durchflussgeschwindigkeitserhöhung von circa $0.2 \% / \mathrm{K}$. Die Wassertemperatur wird durch einen Tempe- 
Kapitel 2. Experimenteller Aufbau des Strahl-Kanten-Systems

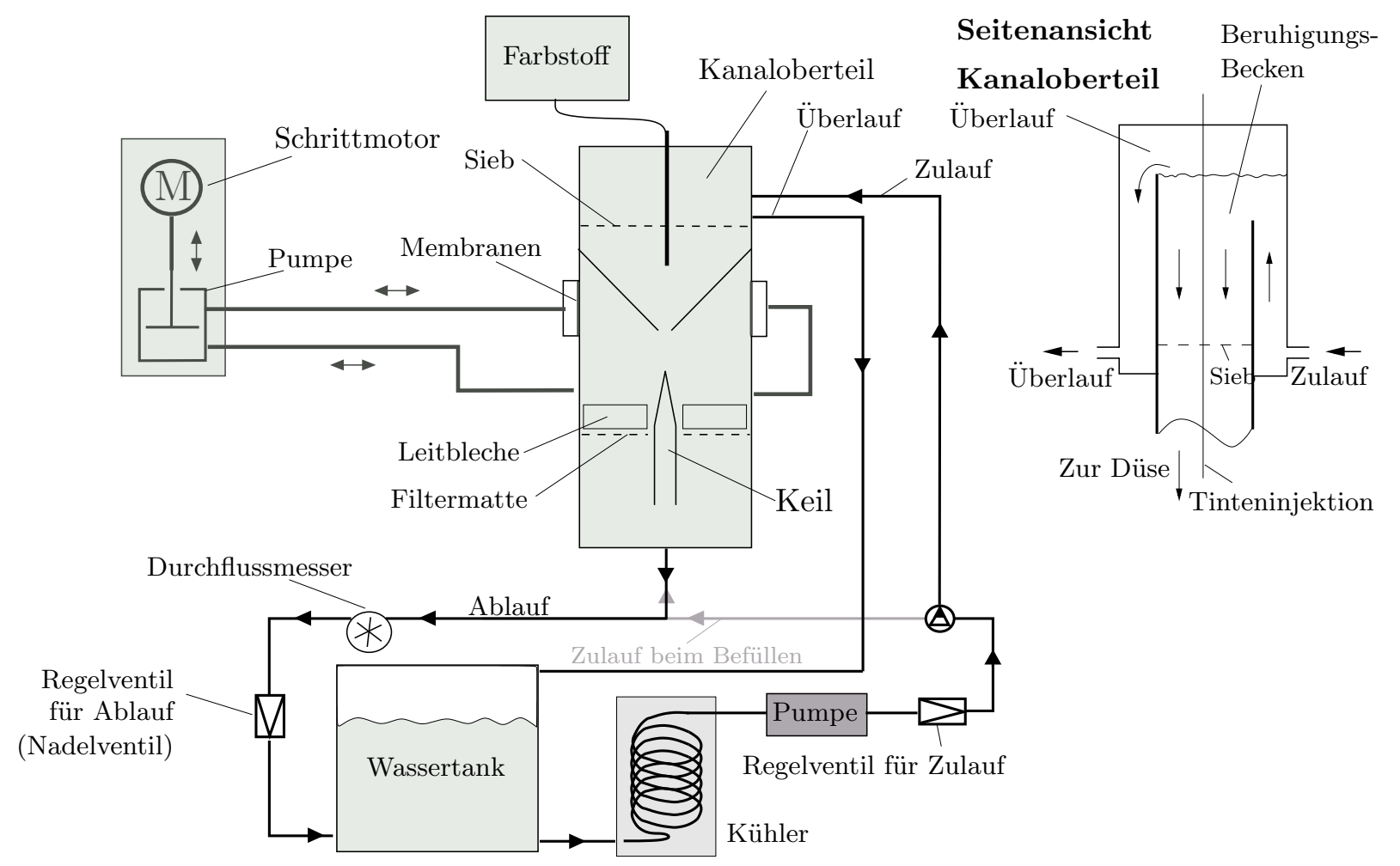

Abb. 2.1: Schematischer Aufbau des Kanals.

raturfühler im Einlauf gemessen und protokolliert. Bei einigen Messungen stand ein Kühler zur Verfügung, der vor der Pumpe eingesetzt werden kann. Das Kanaloberteil mit dem Überlaufbecken ist abnehmbar, so dass zwischen Kanal und Überlaufbecken verschiedene Düsen eingesetzt werden können. Die Düsenbreite $d$ beträgt $2 \mathrm{~mm}$ oder $4 \mathrm{~mm}$. Die Tiefe der Düse (Spannweitenrichtung, z-Achse) beträgt aufgrund der Düsenseitenwände nur 36 mm, während der Kanal $40 \mathrm{~mm}$ tief ist.

Für die Homogenität der Strömung wurden im Einlauf ein Sieb und am Messstreckenende Leitbleche mit dazwischen gelegten Filtermatten angebracht. Die Strömung wurde von PRECKEL mittels LDA vermessen und erwies sich als sehr homogen in Spannweitenrichtung, so dass man die Strömung als zweidimensional bezeichnen kann.

Die Reynoldzahl $\operatorname{Re}_{\mathrm{d}}=U_{\mathrm{D}} d / \nu$, gebildet mit der Durchflussgeschwindigkeit und der Düsenbreite $d$ ( dynamische Viskosität von Wasser $\nu \approx 1 \mathrm{~mm}^{2} / \mathrm{s}^{2}$ ) liegt bei den in dieser Arbeit benutzten Einstellungen meistens zwischen 100 und 250.

\section{Aktuator}

In die Seitenwände des Kanals sind zwei Gummimembranen $\left(3 \times 6 \mathrm{~cm}^{2}\right)$ mit dahinter liegenden wassergefüllten Hohlräumen eingelassen, die durch steife Schläuche mit einer beidseitig wirkenden Kolbenpumpe verbunden sind. Die Kolbenpumpe, die DiERKEN [9] entwickelt hat, 


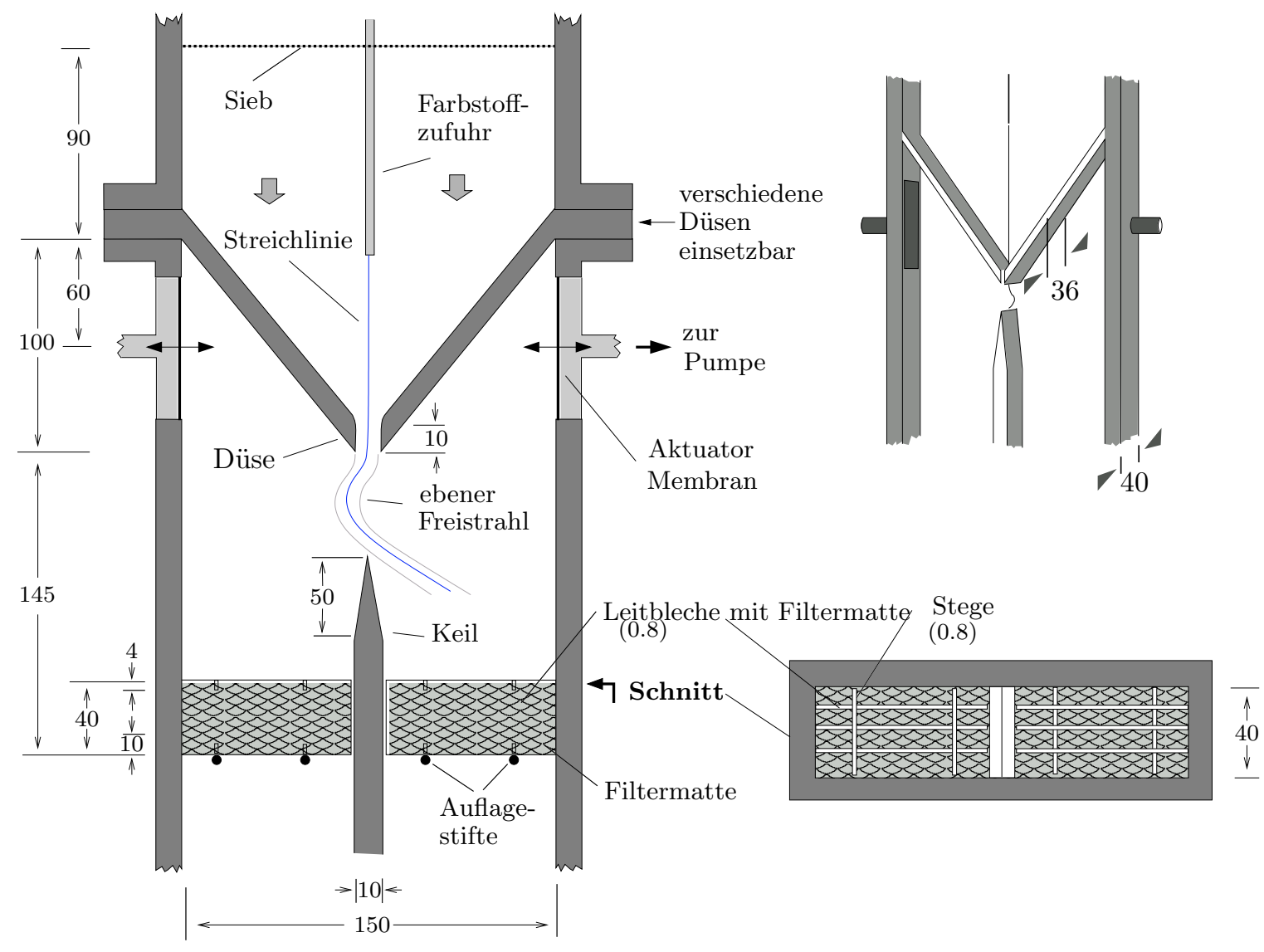

Abb. 2.2: Aufbau des Wasserkanals. Links: Vorderansicht mit Messstrecke (Videoausschnitt beginnt direkt unterhalb der Düse und reicht $4 \mathrm{~cm}$ stromab). Rechts oben: 3-D Ansicht. Rechts unten': Gitter mit Filtermatte als Kanalabschluss.

wird mittels einer Gewindestange durch einen Schrittmotor angetrieben und fördert entgegengesetzt gleiche Volumina. Das garantiert, dass sich die Membranen gleichphasig bewegen. Die Schrittmotor-Steuerung erfolgt durch eine PC-Karte, die speziell für das Experiment entwickelt wurde.

\section{Strömungsbeobachtung}

Zur Sichtbarmachung der Strömung wird eine möglichst nahe der Düsenmitte liegende Streichlinie eingefärbt, die mit einer CCD-Kamera beobachtet werden kann. Der Farbstoff (Methylenblau in einer wässrigen Lösung $(0.9 \mathrm{~g} / \mathrm{l}))$ wird oberhalb der Düse mittels einer Kanüle injiziert. Die Farbstoffzufuhr wird über ein manuelles Infusionssystem geregelt. Durch das Einfärben der mittleren Streichlinie entsteht im Video-Bild ein kleiner Helligkeitseinbruch auf dem helleren Freistrahl, der mittels Computer in Echtzeit ausgewertet werden kann.

Der CCD-Chip (1/2" CCD-Kamera Hitachi KP-M2, Interline-transfer-CCD-Chip) hat eine 
Kapitel 2. Experimenteller Aufbau des Strahl-Kanten-Systems

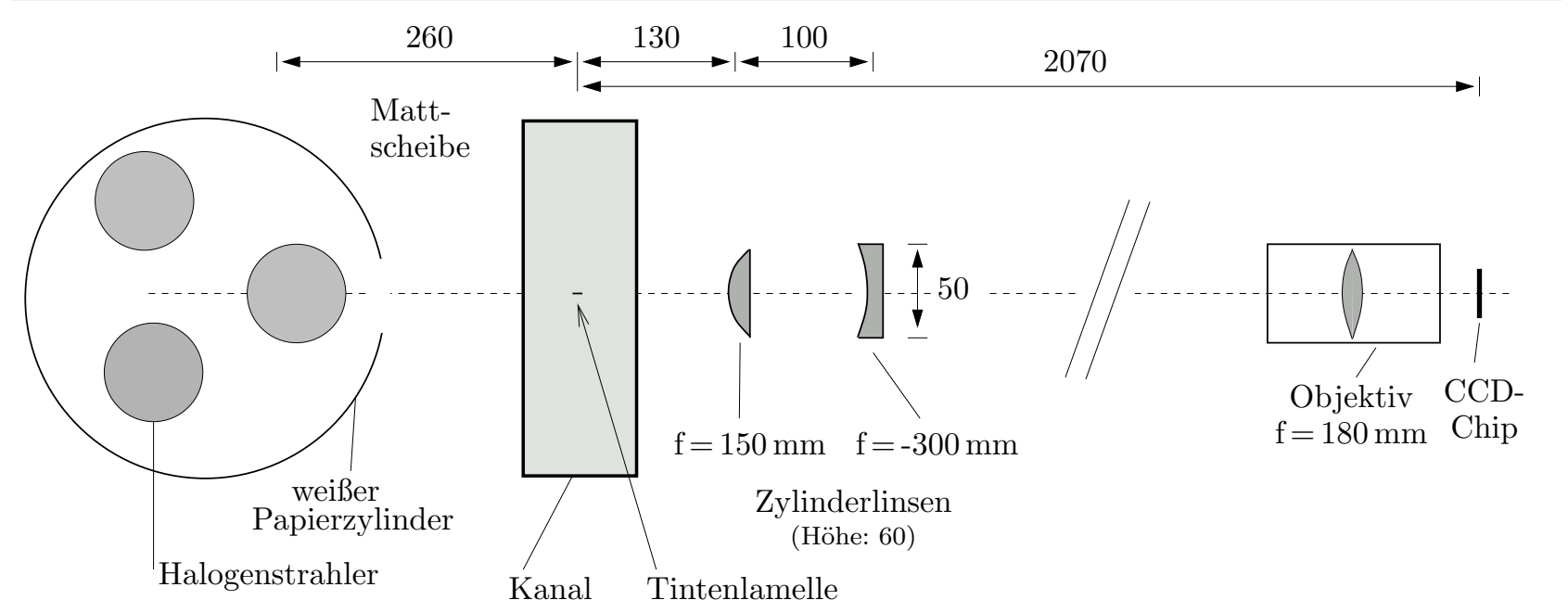

Abb. 2.3: Bildaufnahmesystem mit Zylinderlinsen und „Ulbrichtscher Kugel“ als Beleuchtung. Ein weißer Papierzylinder wird mit drei 20 Watt Halogen-Strahlern von oben beschienen. Im Zylindermantel befindet sich ein rechteckiges Loch, das der Größe des Messbereichs entspricht. Hiermit erreicht man eine Hintergrundbeleuchtung mit einer sehr gleichmäßigen Helligkeitsverteilung.

Bildelementgröße (Pixelgröße) von $8.6 \mu \mathrm{m}$ (horizontal) x $8.3 \mu \mathrm{m}$ (vertikal). Da der Messbereich $(18 \mathrm{~mm}$ x $40 \mathrm{~mm})$ ein anderes Seitenverhältnis als der CCD-Chip hat, kann die Messgenauigkeit in horizontaler Richtung durch Spreizung des Bildes mittels einer anamorphotischen Optik (Abb. 2.3) erhöht werden. Ein zusätzliches Drehen des CCD-Chips hätte die videozeilenorientierte Bildverarbeitung aufwändiger gemacht.

\section{$2.2 \quad$ Regelkreis}

Das Lesen der Videodaten, die Auswertung der Daten und das Ansteuern des Schrittmotors werden durch die $50 \mathrm{~Hz}$ Halbbild-Wiederholfrequenz der Videokamera gesteuert. Die Information der aktuellen Streichlinie, die durch eine sehr kurze Belichtung auf dem CCD-Chip festgehalten wird, führt erst nach einer Totzeit von $60 \mathrm{~ms}$, also 3 Takten, zu einer gewünschten Aktuatorstellung. Die 3 Takte Totzeit mit jeweils 20 ms entstehen durch folgende Schritte:

(1) Übertragung vom CCD-Chip zum Computer Die Halbbilder der CCD-Kamera stehen innerhalb 20 ms nach der Belichtung auf der Framegrabberkarte des Computers zur Verfügung.

(2) Auswertung Der Helligkeitseinbruch wird - beginnend beim Düsenaustritt - stromab verfolgt. Danach kann die Streichlinienposition durch Interpolation auf ca. 1/20 des Pixelabstandes $(\approx 2 \mu \mathrm{m})$ genau bestimmt und ein gewünschtes Regelsignal errechnet werden. 
Alle Rechenschritte müssen innerhalb von 20 ms bis zum nächsten Halbbild erfolgen.

(3) Antialiasingfilter Damit keine Frequenzen über $25 \mathrm{~Hz}$ auf das Videobild bzw. den Strahl übertragen werden, muss das Regelsignal, das den Schrittmotor steuert, mit einem geeigneten Tiefpass-Filter gefaltet werden. Hier dient eine lineare Interpolation zwischen der vorhergehenden und der neuen Aktuatorposition als Tiefpassfilter. Der Schrittmotor, der intern mit einer Taktfrequenz von $4166 \mathrm{~Hz}$ betrieben wird, fährt linear innerhalb von 20 ms zur nächsten Position. Dies entspricht einer Faltung der 83 fach überabgetasteten Sollwerte mit einer Dreiecksfunktion der Breite $20 \mathrm{~ms}$. Das Aktuatorsignal fällt dann zu hohen Frequenzen hin proportional zu $f^{-2}$ ab.

\subsection{Optimale Interpolation der Streichlinienposition aus den Helligkeitswerten der CCD-Kamera}

In jeder Videozeile ist durch die angefärbte Streichlinie ein kleiner Helligkeitseinbruch zu finden. Durch Interpolation der Helligkeitswerte in diesem Helligkeitseinbruch kann die Streichlinienposition auf dem CCD-Chip genauer als der Abstand der Bildelemente berechnet werden. Die CCD-Kamera liefert über die Pixel (Bildelemente) integrierte Helligkeitswerte. Die einzelnen Bildelemente einer Videozeile haben den Pixelabstand $\Delta y_{\text {Pixel }}$ und seien mit $n$ durchnummeriert, so dass die kontinuierlichen Positionen auf dem CCD-Chip durch

$$
y^{*}=n+\Delta y^{*} \text { mit }-0.5<\Delta y^{*} \leq 0.5, \quad y^{*}=y / \Delta y_{\text {Pixel }}
$$

gegeben sind, dabei bedeutet ${ }^{*}$, dass $y^{*}$ die Einheit $\Delta y_{\text {Pixel }}$ hat. Eine Videozeile besteht somit aus den Helligkeitswerten $H(n)$. Das $n$-te Pixel deckt den Bereich $[n-0.5, n+0.5]$ ab. Das wahre bezüglich $y^{*}$ kontinuierliche Helligkeitsprofil $H\left(y^{*}\right)$, der mittels CCD-vermessenen Farbstofflamelle, ist zunächst nicht genau bekannt. In diesem Abschnitt wird das Helligkeitsprofil $H\left(y^{*}\right)$ geschätzt und parametrisiert, um so eine optimale Interpolationsfunktion zur Berechnung der Position $y_{0}^{*}$ der Farbstofflamelle bzw. der Streichlinie zur Verfügung zu stellen.

Die laterale Position $y_{0}^{*}$ der Farbstofflamelle wird aus den Helligkeitswerten $H(n)$ bestimmt, indem zunächst der Ort $n_{0}$ des Intensitätsminimums der $H(n)$ gesucht wird, das über einem bestimmten Pixel durch den Helligkeitseinbruch der Farbstofflamelle entsteht. Die Position $y_{0}^{*}=n_{0}+\Delta y_{0}^{*}$ der Streichlinie kann durch die Helligkeitswerte über den benachbarten Pixeln von $H\left(n_{0}\right)$ interpoliert werden. Es werden nur die beiden Nachbarwerte von $H\left(n_{0}\right)$ bei der Interpolation berücksichtigt, da das Helligkeitsprofil der Farbstofflamelle häufig so schmal ist, dass nur drei benachbarte Pixel $\left[H\left(n_{0}-1\right), H\left(n_{0}\right), H\left(n_{0}+1\right)\right]$ sich mit Sicherheit in ihren Helligkeiten vom Hintergrund abheben. Gesucht ist nun eine optimale Abbildung von $\left[H\left(n_{0}-1\right), H\left(n_{0}\right), H\left(n_{0}+1\right)\right]=:\left[H_{-1}, H_{0}, H_{1}\right]$ auf die gesuchte wahre Streichlinienposition $y_{0}^{*}=n_{0}+\Delta y_{0}^{*}$. 
Kapitel 2. Experimenteller Aufbau des Strahl-Kanten-Systems

Durch eine standardmäßig gewählte Interpolationsfunktion (z.B. polynomiale Funktion, Splines, Schwerpunkt, Gaußkurve) wird das wahre Helligkeitsprofil $H\left(y^{*}\right)$ nur relativ schlecht approximiert und somit $\Delta y_{0}^{*}$ nicht unbedingt getroffen. Es entsteht dann bei gleichverteilten Streichlinienpositionen eine Häufung von $\Delta y_{0}^{*}$ über ganz- oder halbzahligen Werten. Ist die Ansatzfunktion für die Interpolation breiter als das wahre Helligkeitsprofil, so ist die Wahrscheinlichkeitsdichte bei $\Delta y_{0}^{*}=0$ überhöht (siehe Abb. 2.4 (unten)) - eine schmalere Ansatzfunktion führt dagegen zu einer Überhöhung bei $\Delta y_{0}^{*}= \pm 0.5$.

Dieser sogenannte Peaklocking-Effekt kann durch die Kenntnis des wahren Helligkeitsprofils beseitigt werden. Dieses ist abhängig vom Farbstoffzufluss, der Strömungsgeschwindigkeit (siehe Abb. 2.5), der Trübung des Wassers, der Beleuchtungsstärke, dem Abstand von der Düse sowie dem horizontalen Blickwinkel der Kamera, da die Farbstofflamelle im Querschnitt elliptisch (1:10) ist, so dass trotz einer nur kleinen Parallaxe $\left(0.3^{\circ}\right)$ die Farbstofflamelle, seitlich gesehen, ein leicht anderes Helligkeitsprofil aufweist.

Um die richtige Interpolationsfunktion zu finden, muss das kontinuierliche Helligkeitsprofil der Farbstofflamelle gefunden werden. Das annähernd als symmetrisch angenommene Helligkeitsprofil kann durch folgende Annahmen approximimiert werden:

- Hat das Helligkeitsminimum $H_{0}=H\left(n_{0}\right)$ mit einem seiner Nachbarn $H_{1}=H\left(n_{0}+1\right)$ oder $H_{-1}=H\left(n_{0}-1\right)$ die gleiche Intensität, so befindet sich das Minimum bei $y_{0}^{*}=$ $n_{0} \pm$ 0.5. Die Helligkeiten $\left[H_{-1}, H_{0}, H_{1}\right]$ gehören somit für $H_{-1} \approx H_{0}$ zu den Positionen $\left[n_{0}-0.5, n_{0}+0.5, n_{0}+1.5\right]$ und für $H_{1} \approx H_{0}$ zu $\left[n_{0}-1.5, n_{0}-0.5, n_{0}+0.5\right]$. Mittelt man über derartige Tripel, erhält man ungefähr die Helligkeitswerte an den Orten $\left[n_{0}-\right.$ $\left.1.5, n_{0}-0.5, n_{0}+0.5, n_{0}+1.5\right]$.

- Haben die beiden Nachbarn des Helligkeitsminimums die gleiche Intensität $\left(H_{-1} \approx H_{1}\right)$, so befindet sich die Streichlinie am Ort des Helligkeitsminimums, d.h. zu dem Helligkeitstripel $\left[H_{-1}, H_{0}, H_{1}\right]$ gehören die Positionen $\left[n_{0}-1, n_{0}, n_{0}+1\right]$. Mittelt man über alle Tripel, die dieser Bedingung genügen, erhält man die Helligkeit an den Orten $\left[n_{0}-1, n_{0}, n_{0}+1\right]$.

- Aus diesen sieben Helligkeitswerten wird eine stückweise lineare Helligkeitsverteilung $H_{\text {interp }}\left(y^{*}-y_{0}^{*}\right)$ interpoliert. Einem gemessenen Helligkeitstripel $\left[H_{-1}, H_{0}, H_{1}\right]$ wird dann der Ort $y_{0}^{*}$ so zugeordnet, dass der euklidische Abstand zwischen gemessenen und dem entsprechendem interpolierten Helligkeitstripel minimal wird:

$$
y_{0}^{*} \in[-0.5,0.5]: \sum_{i=-1}^{1}\left(H_{\text {interp }}\left(n_{0}+i-y_{0}^{*}\right)-H_{\mathrm{i}}\right)^{2}=\min .
$$

Die so umgeordneten Helligkeitswerte sind in Abb. 2.5 für verschiedene Strömungsgeschwindigkeiten und Tintenflüsse interpoliert worden. 

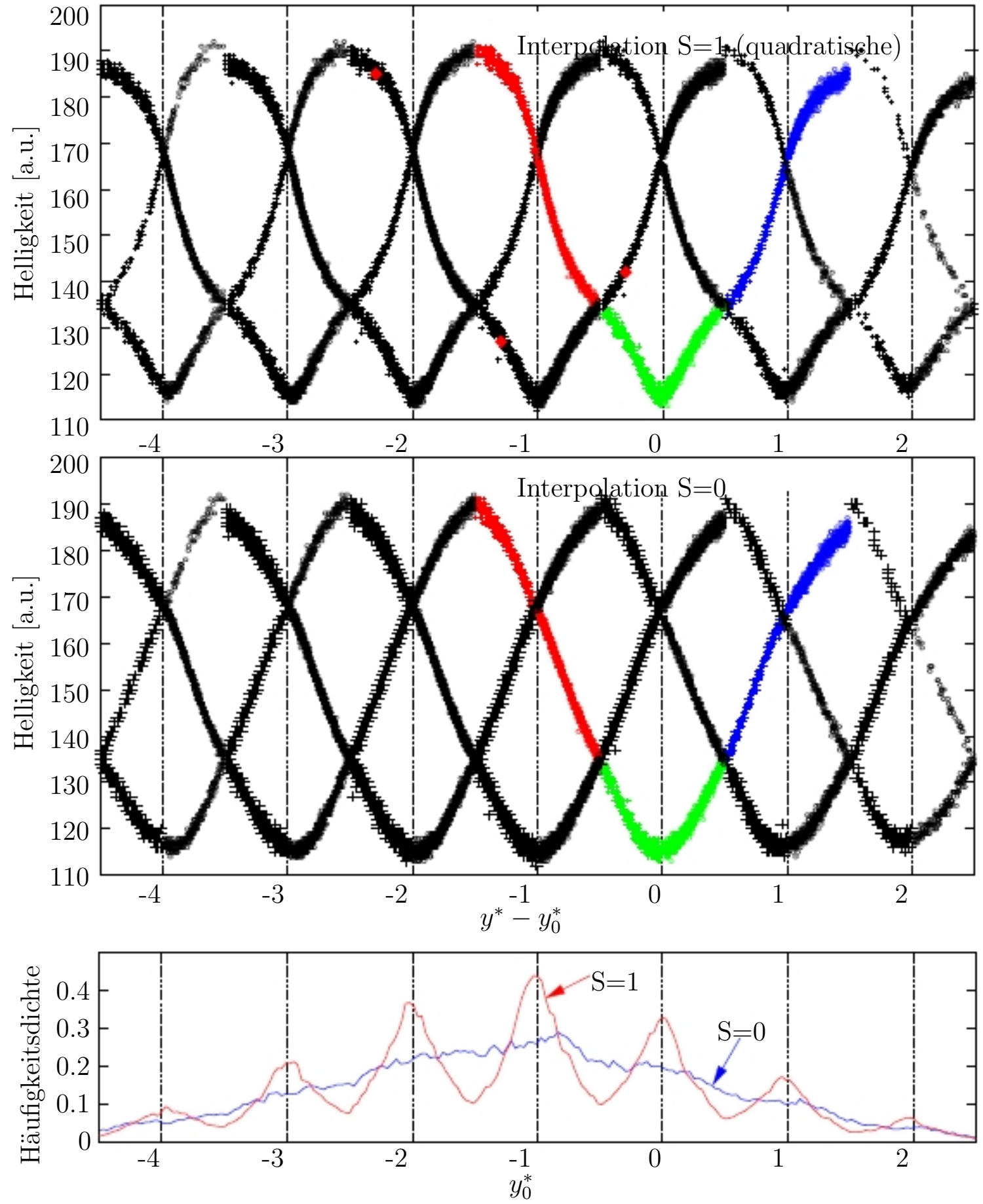

Abb. 2.4: Position der Helligkeitstripel bei verschiedenen Ansatzfunktionen innerhalb des Helligkeitsprofils. Oben: quadratische Ansatzfunktion, entspricht $S=1$ in Gl. (2.7). In den Farben rot, grün, blau sind $\left[H_{-1}, H_{0}, H_{1}\right]$ dargestellt. Mitte: korrigierte Minima durch Ansatzfunktion $S=0$. Unten: die daraus entstehende Häufigkeitsdichte von $y_{0}^{*}$. 


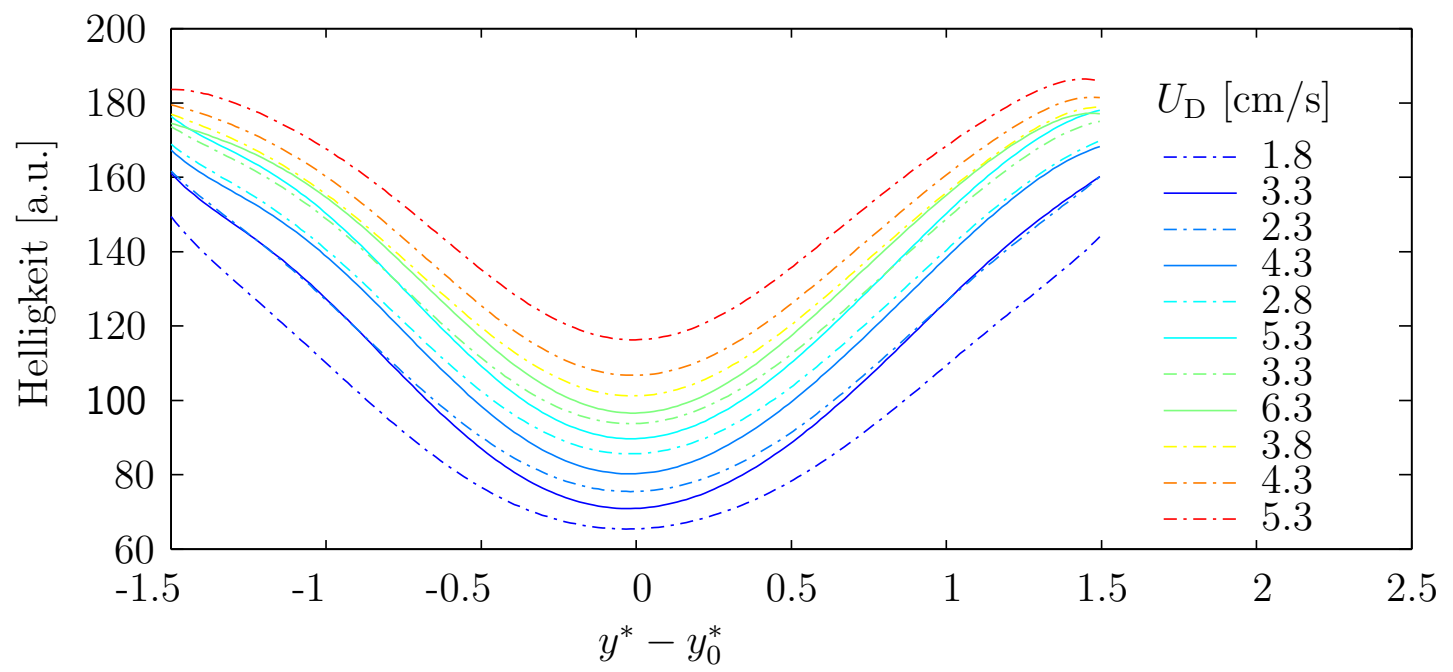

Abb. 2.5: Helligkeitsprofile der Farbstofflamelle. Interpolierte Helligkeitsprofile aus den umgeordneten Helligkeitswerten der Farbstofflamelle. Für verschiedene Strömungsgeschwindigkeiten und zwei unterschiedliche Tintenflüsse $(6.3 \mathrm{ml} /$ Stunde (durchgezogene Linien) und $12 \mathrm{ml} /$ Stunde (gestrichelte Linien)). Bei langsamer Strömungsgeschwindigkeit lässt die Diffusion des Farbstoffs die Helligkeitsprofile breiter werden.

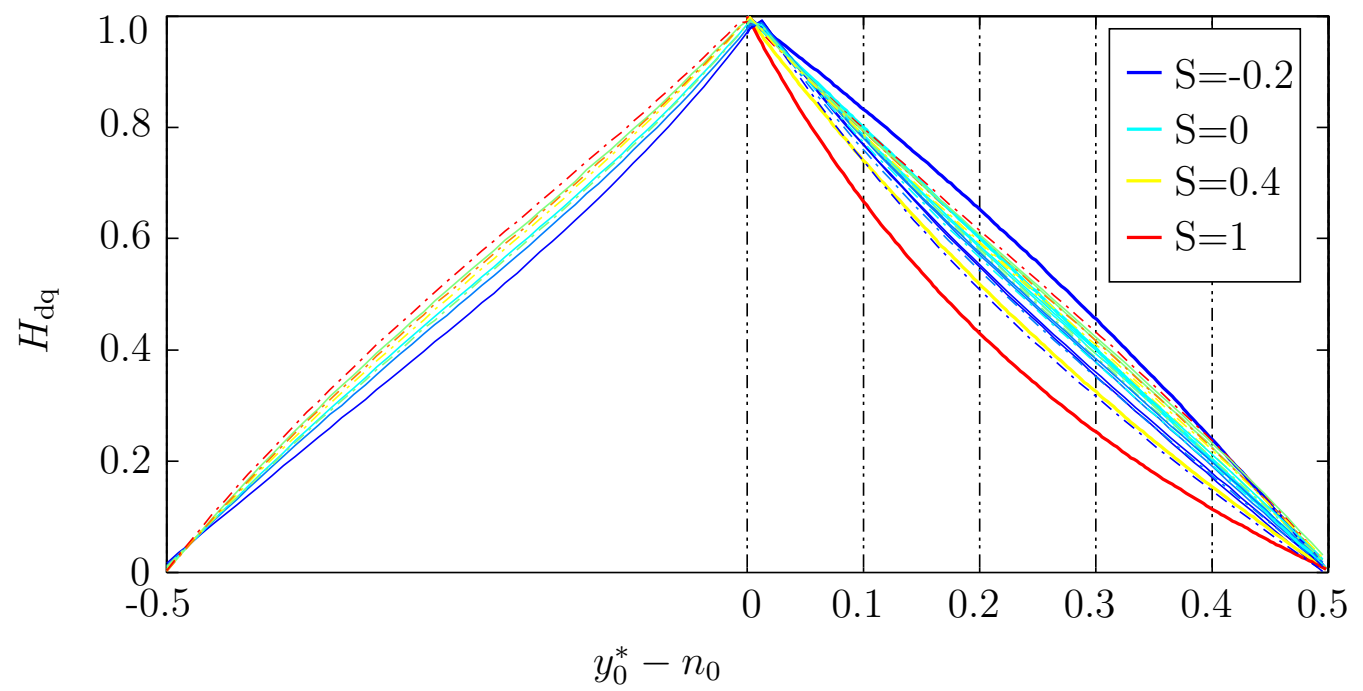

Abb. 2.6: Quotient der Helligkeitswerte $\boldsymbol{H}_{\text {dq }}$. Rechte und linke Seite: $H_{\mathrm{dq}}$ (siehe Gl. (2.3)) mit Legende aus Abb. 2.5. Rechte Seite: zusätzlich Interpolationsschar mit Parameter $S$ (siehe dazu Gl. (2.7)). 
Ein einheitlicheres Bild ergibt sich, wenn man den Quotienten der Helligkeitsdifferenzen zwischen den Nachbarn und des Minimums betrachtet (siehe Abb. 2.6):

$$
H_{\mathrm{dq}}=\left\{\begin{array}{lll}
\frac{H_{-1}-H_{0}}{H_{1}-H_{0}} & \text { für } H_{-1}<H_{1} & y_{0}^{*}-n_{0}<0 \\
\frac{H_{1}-H_{0}}{H_{-1}-H_{0}} & \text { für } H_{-1}>H_{1} & y_{0}^{*}-n_{0}>0 .
\end{array}\right.
$$

Dieser Quotient ist unabhängig von den relativ willkürlichen Parametern der Helligkeit am Ort der Farbstofflamelle $H\left(n_{0}\right)$ und der Helligkeit des umgebenden Strahls $H(\infty)$ und ist daher für die Bestimmung von $\Delta y_{0}^{*}=y_{0}^{*}-n_{0}$ besonders geeignet.

Betrachtet man Abb. 2.6, so stellt man fest, dass $H_{\mathrm{dq}}$ annähernd der Beziehung

$$
H_{\mathrm{dq}}=1-2\left|\Delta y_{0}^{*}\right|
$$

genügt, die leicht invertiert werden kann:

$$
\Delta y_{0}^{*}= \pm \frac{1-H_{\mathrm{dq}}}{2} .
$$

Betrachtet man eine quadratische Funktion als Ansatzfunktion für das Helligkeitsprofil, so gilt für das Minimum

$$
y_{0}^{*}=n_{0} \pm \frac{1-H_{\mathrm{dq}}}{2\left(1+H_{\mathrm{dq}}\right)},
$$

und es erweist sich als sinnvoll, verallgemeinernd die Schar von Umkehrfunktionen mit Parameter $S$

$$
y_{0}^{*}=n_{0} \pm \frac{1-H_{\mathrm{dq}}}{2\left(1+S \cdot H_{\mathrm{dq}}\right)}
$$

zu betrachten. Diese Schar ist auf der rechten Seite von Abb. 2.6 zusammen mit den Quotienten der Differenzen der Helligkeitswerte aufgetragen und deckt den Bereich der vorkommenden Kurven gut ab. Da das geschätzte Helligkeitsprofil leicht asymetrisch ist, sind die Kurven in Abb. 2.6 links und rechts unterschiedlich.

In Abb. 2.4 (oben) wird für eine Beispielmessung der Ort des Minimums $y_{0}^{*}$ durch eine quadratische Ansatzfunktion $(S=1)$ für das Helligkeitsprofil bestimmt. Die Häufigkeitsdichte von $y_{0}^{*}$ ist in der Abbildung unten dargestellt. Verschiebt man das jeweilige Helligkeitstripel um $-\Delta y_{0}^{*}$, so ergibt sich daraus das zugrunde gelegte Helligkeitsprofil. Der Abstand nach Gl. (2.2) zu $H_{\text {Interp }}$ ist dabei nicht optimal. Mit einer Ansatzfunktion der neuen Interpolationsformel $S=0$ ist das sich daraus ergebende Profil in Abb. 2.4 (Mitte) gezeigt. Die Wahrscheinlichkeitsdichte zu $S=0$ zeigt keine ausgeprägten Peaks mehr und die Anordnung der Helligkeitstripel ist im Sinne von Gl. (2.2) optimal. 


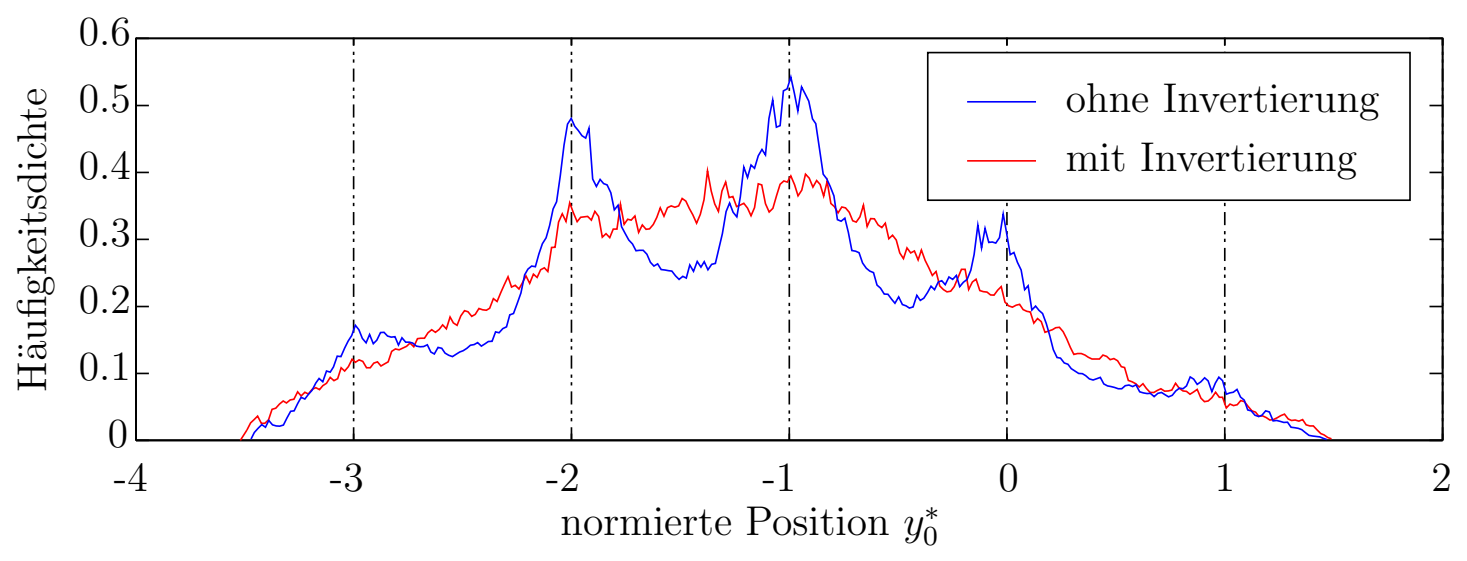

Abb. 2.7: Statistische Invertierung des Peak-Lockings für $S=1$.

\section{Statistische Korrektur des Interpolationsfehlers bei der Positionsbestimmung der Streichlinie}

Zu der im letzten Abschnitt gefundenen Parametrisierung des Helligkeitsprofils der Farbstofflamelle kann zusätzlich der Interpolationsfehler bei der Positionsbestimmung (Peak-Locking) noch direkt statistisch invertiert werden. Dies ist notwendig, da zum einen die Trübung des Wassers im Laufe der Messzeit zunimmt und zum anderen verschiedene Strömungsgeschwindigkeiten und Tintenflüsse zu verschiedenen Helligkeitsprofilen führen. Daher steht nicht immer die beste Interpolationsfunktion bei gewählter Parametrisierung $S$ des Helligkeitsprofils zur Verfügung. Eine Auswertung in Echtzeit, die ständig den Parameter $S$ nach dem Schema des Abschnitts 2.3 bestimmt, würde sehr viel Rechenzeit kosten. Außerdem ist das wahre Helligkeitsprofil trotz der näherungsweise parallaxenfreien Aufnahme leicht von der horizontalen Pixelposition $n_{0}$ abhängig. Es kann daher versucht werden, eine in Echtzeit mitlaufende statistische Korrektur der Positionen vorzunehmen. In Abb. 2.7 ist die Häufigkeitsdichte der interpolierten Streichlinienposition durch eine quadratische Ansatzfunktion $(S=1)$ des Helligkeitsprofils der Farbstofflamelle gezeigt, die ausgeprägte Spitzen bei $y_{0}^{*}-n_{0}=0$ aufweist. Gesucht ist nun eine Abbildung, die den Positionen $y_{0}^{*}$ neue, korrigierte Positionen $y_{0 \mathrm{korr}}^{*} \mathrm{zu}$ ordnet, so dass die Ungleichverteilung in Abb. 2.7 verschwindet.

Das Problem, diese Abbildung zu bestimmen, liegt in der Trennung der Gesamtstatistik aller Pixel einer Videozeile von der Statistik eines einzelnen Pixels. Läge zum Beispiel der Umkehrpunkt einer sinusförmigen Auslenkung der Streichlinienposition innerhalb eines Pixels, so würde ein Sprung in der Häufigkeitsdichte entstehen, der nicht invertiert (und somit geglättet) werden darf. Um die Pixelstatistik von der Gesamtstatistik zu trennen, kann wie folgt vorgegangen werden:

Jedes normierte Pixelintervall $[n-0.5, n+0.5]$ (Gl. (2.1)) wird in 100 Teilintervalle eingeteilt, in die die ermittelten $y_{0}^{*}$ eingeordnet werden. Ist eine gewisse statistische Sicherheit erreicht, 


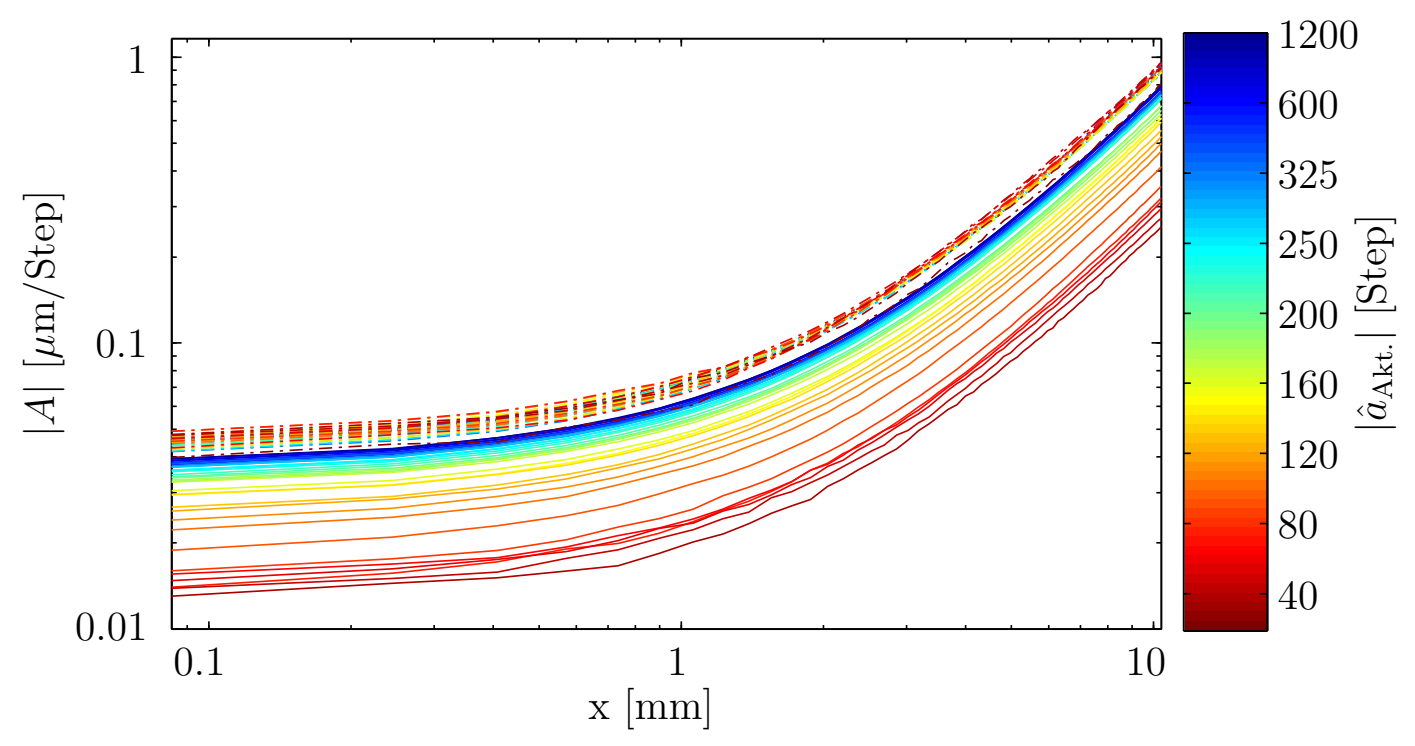

Abb. 2.8: Betrag des Aktuatorübertragungsfaktors $|\boldsymbol{A}|$ als Funktion der Stromabposition $x$ mit Hystereseinvertierung (gestrichelt) und ohne Hystereseinvertierung. Der Parameter Aktuatoramplitude $\left|\hat{a}_{\text {Akt. }}\right|$ wird durch die seitliche Farbskala den Farben der Kurven zugeordnet. Strouhalzahl $\mathrm{Sr}_{\mathrm{d}}=0.73$.

werden die Grenzen der Teilintervalle so verschoben, dass die Häufigkeitsdichte über dem PixelIntervall konstant wird; dies geschieht allerdings nur, wenn die Gesamthäufigkeiten in dem betrachteten und den beiden benachbarten Pixel-Intervallen nur um maximal $10 \%$ differieren. So kann man relativ sicher sein, dass die Aufenthaltswahrscheinlichkeit des Tintenfadens in dem betrachteten Pixel-Intervall tatsächlich gleichverteilt ist. Als Maß für die Sicherheit der Schätzung der Verteilung wird hier aus Rechenzeitgründen einfach eine bestimmte Anzahl von Treffern (30-60) im mittleren Teilintervall des mittleren Pixel-Intervalls benutzt. Andere Maße wie die „Glätte der Verteilung“ oder die „Gesamtanzahl der Treffer" können nicht ohne zusätzliche zeitintensive Rechenoperationen abgefragt werden. Bei der Invertierung werden den alten 100 Teilintervallen gleichverteilte 20 neue Teilintervalle zugeordnet. Anschließend werden die 20 wieder auf 100 linear interpoliert, so dass eine möglichst glatte Inverse entsteht. Als Beispiel für eine Invertierung ist in Abb. 2.7 ohne und mit Online-Invertierung gemessen worden.

\subsection{Hysterese der Aktuatoranregung}

Bei Messungen mit sehr kleinen Aktuatoramplituden (siehe Abschnitt 5.3.3) wurde festgestellt, dass das Verhältnis zwischen der Streichlinienauslenkung (Messsignal) und dem verursachenden Aktuatorsignal sehr klein wird, was zur Instabilität einer adaptiven Regelung führen kann. Daher wird die Beziehung zwischen Aktuatorsignal und Streichlinienauslenkung hier detailliert untersucht. 
Der in Abb. 2.8 aufgetragene Betrag des Aktuatorübertragungsfunktion $|A|$ :

$$
A\left(x, \hat{a}_{\mathrm{Akt} .}\right):=\frac{\hat{\eta}\left(x, \hat{a}_{\mathrm{Akt} .}\right)}{\hat{a}_{\mathrm{Akt} .}}
$$

nimmt für kleinere Aktuatoramplituden $\left|\hat{a}_{\text {Akt. }}\right|$ deutlich ab. Die Aktuatorauslenkung ist dabei die Eingangsgröße des Schrittmotors und wird in Steps gemessen (siehe Anhang C.1). Der Schrittmotor bewegt über eine Pumpe die Membranen, deren Auslenkung bzw. verdrängtes Volumen soll möglichst genau proportional zur Aktuatorauslenkung sein. Dies ist jedoch nur für größere Aktuatoramplituden der Fall. Da eine direkte Messung der Membranauslenkung, bzw. des von ihr verdrängten Volumens nicht möglich ist, soll die erzeugte Streichlinienauslenkung als direktes Maß für die Membranauslenkung genutzt werden. Die Streichlinienauslenkungsamplitude $\hat{\eta}\left(x, \hat{a}_{\mathrm{Akt}}.\right)$ setzt sich zum einen aus dem direkt von den Membranen induzierten Feld und zum anderen aus der Entwicklung der Instabilitätswelle zusammen. Als charakteristische Messgröße der Membranbewegung, die durch die effektive Aktuatorbewegung erzeugt wird, kann $\hat{\eta}$ nur im Düsennahbereich dienen, da dort der nichtlinear von $\hat{a}_{\text {Akt. }}$ abhängige Anteil der Instabilitätswelle vernachlässigbar ist (Abschnitt B.1.2). Daher zeigen die zu verschiedenen $\hat{a}_{\text {Akt. }}$. gemessenen $A\left(x, \hat{a}_{\mathrm{Akt}}\right)$ eine im wesentlichen identische $x$-Abhängigkeit. Für verschiedene Anregungsfrequenzen und Strömungsgeschwindigkeiten erhält man ähnliche Abhängigkeiten des mittleren Übertragungsfaktors $\langle A\rangle_{\mathrm{x}<1.2 \mathrm{~mm}}$ von der Aktuatorauslenkung (siehe Abb. 2.9).

Hier soll für die quantitative Beschreibung von $A\left(\hat{a}_{\mathrm{Akt}}\right)$ ein einfaches Modell einer statischen Hysterese angenommen werden: Die Aktuatoramplitude $\hat{a}_{\text {Akt. }}$ wird dazu in einen effektiven

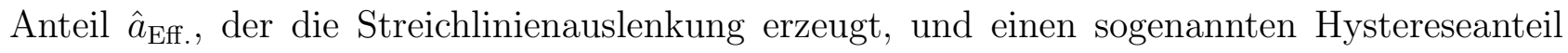
$\hat{a}_{\text {Hyst. }}$, der den Freilauf bzw. die verminderte Effekitivität des Aktuators bei kleinen Amplituden beschreibt, zerlegt:

$$
\hat{a}_{\mathrm{Akt} .}=\hat{a}_{\mathrm{Eff} .}+\hat{a}_{\mathrm{Hyst} .},
$$

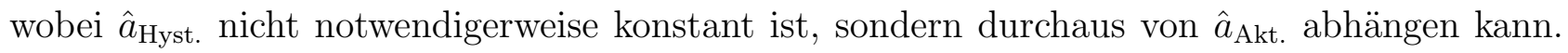
Erst mit größer werdendem $\hat{a}_{\text {Akt. }}$ wird angenommen, dass der Freilauf vollständig durchfahren ist, und somit $\hat{a}_{\text {Hyst. }}$ konstant wird. Der effektive Anteil $\hat{a}_{\text {Eff. erzeugt eine Streichlinienauslen- }}$ kung:

$$
\hat{\eta}=A^{\text {asymp. }} \cdot \hat{a}_{\mathrm{Eff} .}\left(\hat{a}_{\mathrm{Akt} .}\right)
$$

die durch einen unbekannten Übertragungsfaktor $A^{\text {asymp. }}$ gegeben ist. Da mit steigender Ak-

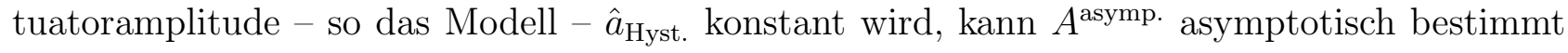
werden:

$$
A^{\text {asymp. }}:=\lim _{\left|\hat{a}_{\mathrm{Akt} .}\right| \rightarrow \infty} \frac{\hat{\eta}\left(\hat{a}_{\mathrm{Eff} .}\left(\hat{a}_{\mathrm{Akt} .}\right)\right)}{\hat{a}_{\mathrm{Akt} .}} .
$$




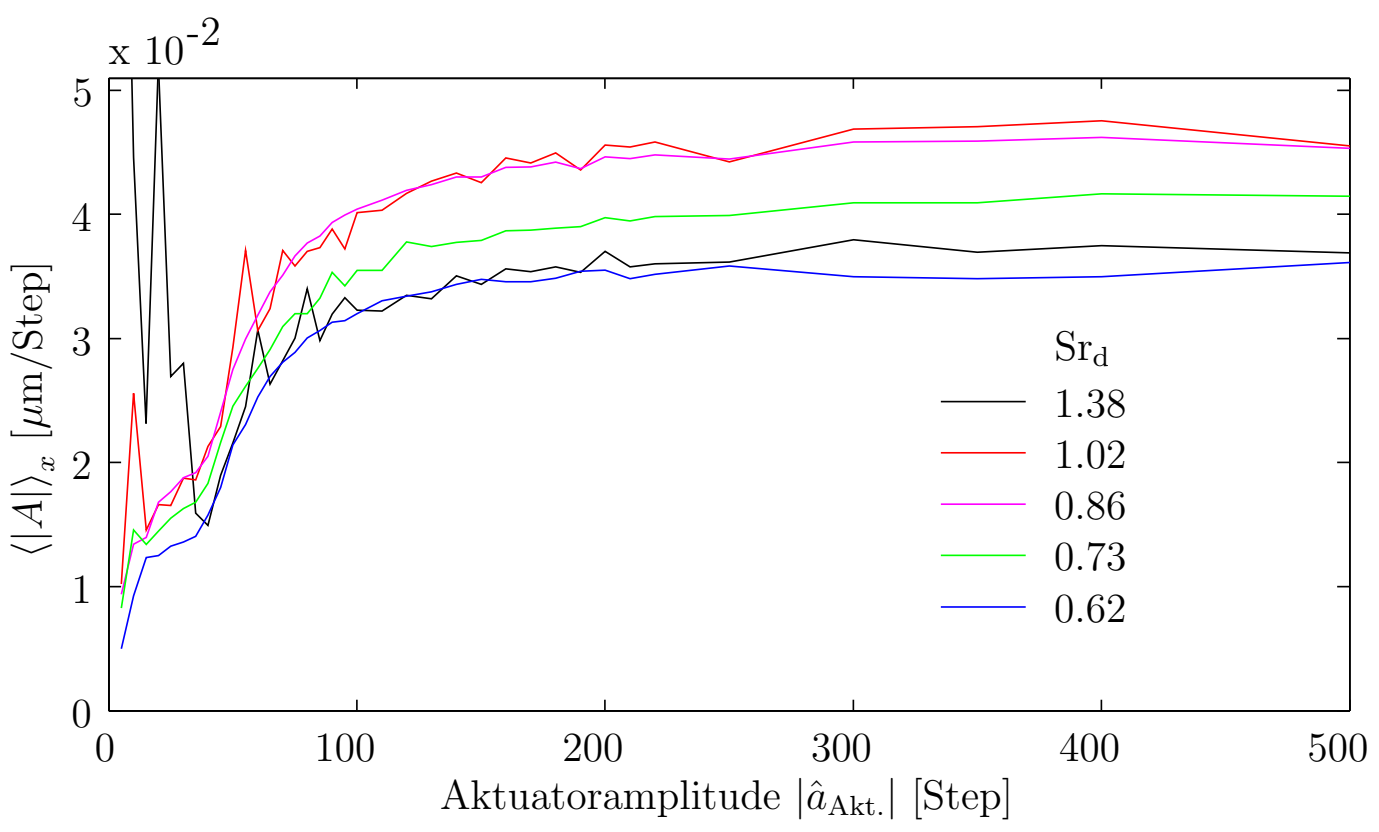

Abb. 2.9: Aktuatorübertragungsfaktor gegen Aktuatorauslenkungsamplitude im Düsennahbereich $x<1.2 \mathrm{~mm}$ gemittelt, für verschiedene Strouhalzahlen $\mathrm{Sr}_{\mathrm{d}}$. Die Messung mit $\mathrm{Sr}_{\mathrm{d}}=1.38$ wurde durch eine sehr langsame Strömungsgeschwindigkeit realisiert, so dass sich dort die Farbstofflamelle stark verbreitert hat. Die kleinen Auslenkungen $(<1 / 100 \mathrm{~mm})$ lassen sich nur mit Unsicherheit bestimmen.

Aus Gleichung 2.9 folgt dann, unter Vernachlässigung von Phasenunterschieden bei den Übertragungsfaktoren, für die Hysterese des Aktuators:

$$
\begin{aligned}
\left|\hat{a}_{\text {Hyst. }}\left(\hat{a}_{\text {Akt. }}\right)\right| & =\left|\hat{a}_{\text {Akt. }}\right|-\left|\hat{\eta} / A^{\text {asymp. }}\right|=\left(1-\left|\frac{\hat{\eta}}{\hat{a}_{\text {Akt. }}} / A^{\text {asymp. }}\right|\right) \cdot\left|\hat{a}_{\text {Akt. }}\right| \\
& =\left(1-\left|\frac{A\left(\hat{a}_{\text {Akt. }}\right)}{A_{\text {asymp. }}}\right|\right) \cdot\left|\hat{a}_{\text {Akt. }}\right| .
\end{aligned}
$$

Der in Abbildung 2.10 dargestellte Hystereseanteil $\hat{a}_{\text {Hyst. }}$ als Funktion der gesamten Aktuatoramplitude $\hat{a}_{\text {Akt. }}$ steigt zunächst annähernd linear an und wird dann konstant. Somit können zwei lineare Funktionen benutzt werden, um die Hysterese zu beschreiben. Bezüglich der Aktuatorauslenkung ergibt sich dann das Hysteresediagramm in Abb. 2.10.

Aus der gefundenen Hysterese kann ein invertiertes Signal $a_{\text {invertiert }}(t)$ für den Schrittmotor berechnet werden, so dass der Ort der Membranen genau das Soll-Signal $a_{\text {Soll }}(t)$ erreicht. Zur Berechnung wird die Soll-Position der letzten Umkehr $a_{\text {Soll }}\left(t_{\text {Umkehr }}\right)$ und die dort zum Zeitpunkt $t_{\text {Umkehr }}$ addierte Korrektur $a_{\text {Korrektur }}\left(t_{\text {Umkehr }}\right)$ gespeichert. Entsprechend $\left|a_{\text {Soll }}\left(t_{\text {Umkehr }}\right)-a_{\text {Soll }}(t)\right|$ wird die Korrektur für den Schrittmotor durch die ermittelte Hysterese berechnet.

Abbildung 2.11 zeigt das invertierte Signal $a_{\text {invertiert }}(t)$ für ein synthetisches Dreieckssignal 


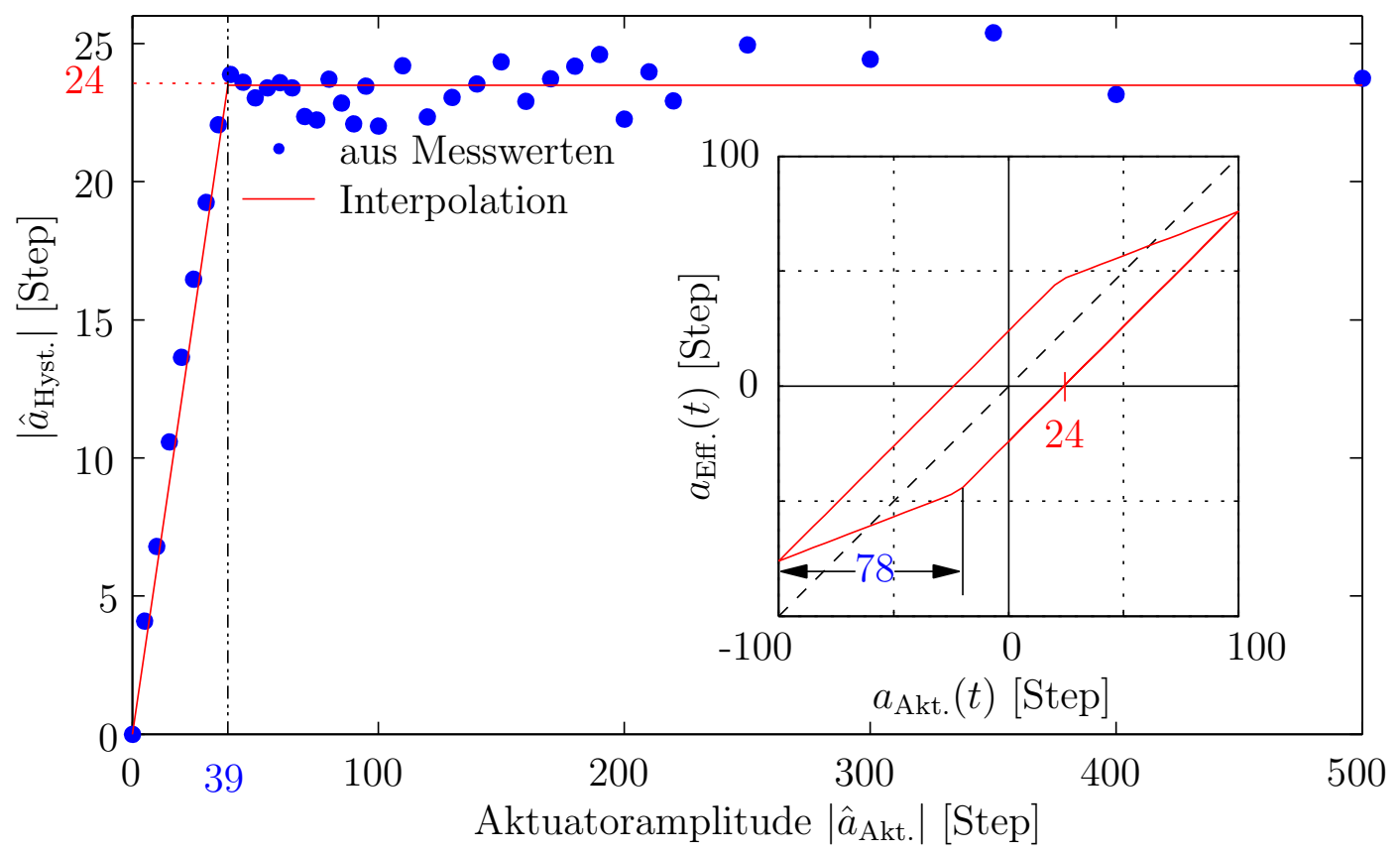

Abb. 2.10: Hysterese. Aus den Wechselamplituden bestimmte Hystereseamplitude (siehe

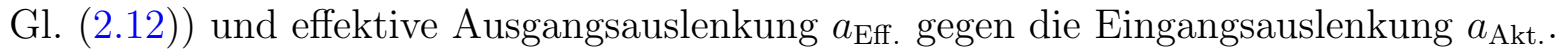

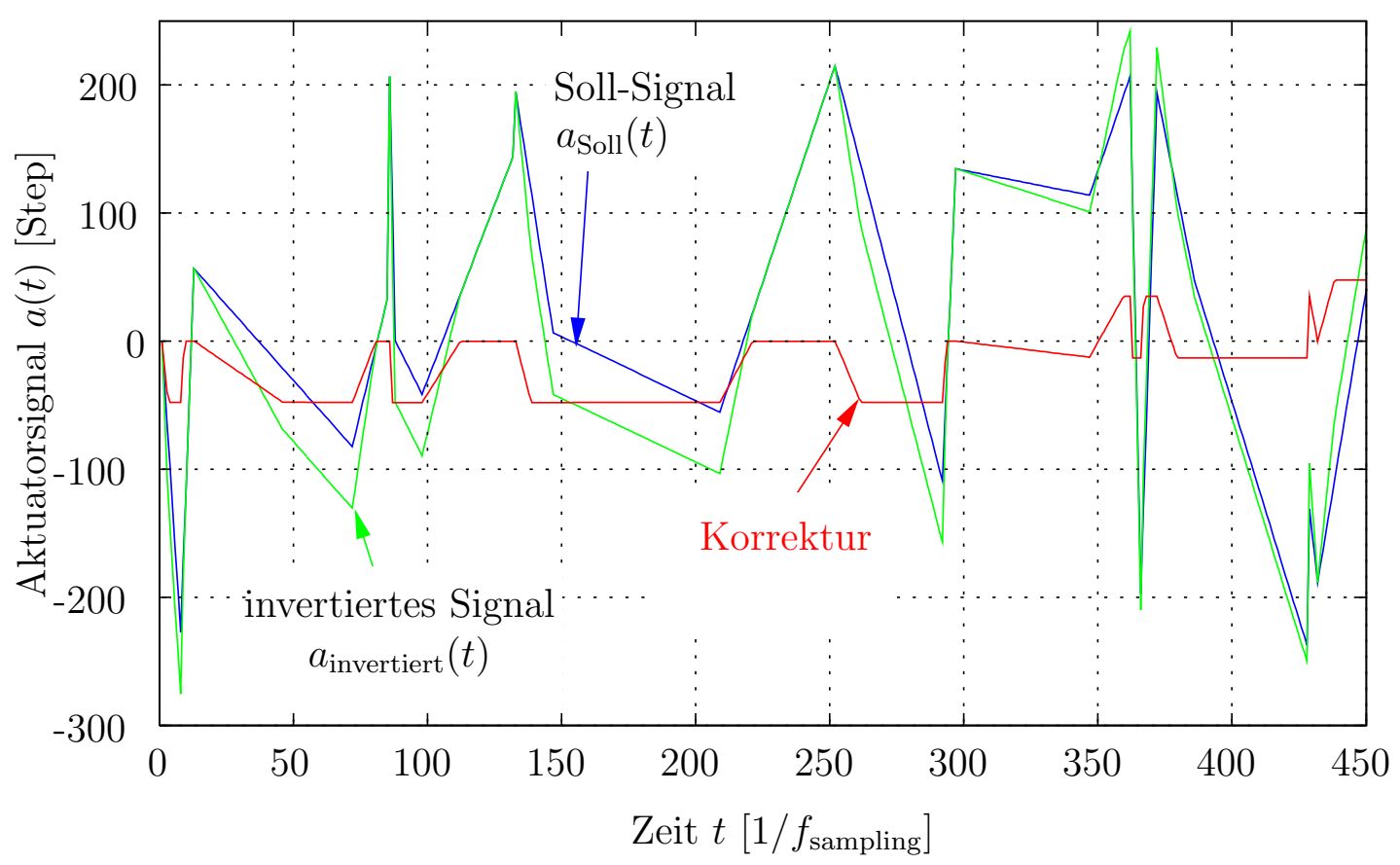

Abb. 2.11: Hystereseinvertierung. Soll-Signal der Membranauslenkung und invertiertes Signal zur Ansteuerung des Schrittmotors. 
$a_{\text {Soll }}(t)$, das durch die Korrektur für den Schrittmotor aufgearbeitet wurde. Die in den Umkehrpunkten benutzte Korrektur $a_{\text {Korrektur }}\left(t_{\text {Umkehr }}\right)$ muss dabei berücksichtigt werden, da die Aktuatoren sonst an diesen Punkten einen Sprung machen würden. Bei der Implementation wurde die aktuelle Aktuatorposition innerhalb des Freilaufs nicht berücksichtigt, so dass es bei kleinen asymetrischen Aktuatorauslenkungen zu einer langsamen Verschiebung der Nullposition des Schrittmotors kommen kann; in Abb. 2.11 wird dies bei $t \cdot f_{\text {sampling }}=350$ deutlich. Daher wird die Korrektur durch einen nahe bei eins gelegenen Vergessensfaktor auf kontinuierlich Null hingeführt. 



\section{Kapitel 3}

\section{Modellierung der konvektiven Instabilität}

Die konvektive Instabilität des Freistrahls führt zusammen mit der Strahl-Kanten-Wechselwirkung zu der in Kap. 4 detailliert beschriebenen globalen Instabilität. Die dort beschriebenen Wechselwirkungen basieren auf dem Geschwindigkeitsfeld der Freistrahlinstabilität. Die gemessene Streichlinie integriert über dieses und führt zu einer schwierigen Vermischung von Gleichströmungsfeld und Instabilitätswellenfeld. In diesem Kapitel wird die konvektive Instabilität und deren Sättigung mit wachsender Anregungsamplitude modelliert. Durch Integration der Streichlinie kann unter anderem auch der experimentell gefundene Widerspruch aufgelösst werden, dass die Phasendrehung pro Lauflänge der Streichlinie mit wachsender Anregungsamplitude abnimmt, während die der konvektiven Instabilität zunimmt.

\subsection{Rayleigh-Gleichung}

Zunächst sei eine reine Parallelströmung mit dem Hauptströmungsprofil $\bar{U}(y)$ betrachtet. Wird die Parallelströmung an einer Stelle zeitlich sinusförmig mit der Kreisfrequenz $\omega_{\mathrm{s}}$ und mit infinitesimaler Amplitude gestört, so lässt sich im Sinne der klassischen Stabilitätstheorie die Stromfunktion $\Phi$ in genügendem Abstand stromab der Störstelle und in einem gewissen Frequenzbereich als Summe eines zeitlich gemittelten Teils

$$
\bar{\Phi}=\int_{0}^{y} \bar{U}\left(y^{\prime}\right) d y^{\prime}
$$

und einer Welle mit der Wellenlänge $2 \pi / \mathfrak{R e}\{k\}$ sowie der räumlichen Anfachung $\mathfrak{I m}\{k\}$ (in komplexer Schreibweise) darstellen:

$$
\Phi(x, y, t)=\bar{\Phi}(y)+\tilde{\Phi}(x, y, t)=\bar{\Phi}(y)+\hat{\Phi}(y) \cdot e^{i\left(\omega_{s} t-k x\right)} .
$$

Die zeitliche Entwicklung der Phase der Instabilitätswelle wird hier - wie in der Signaltheorie üblich - durch $e^{+i \omega_{\mathrm{s}} t}$ beschrieben, so dass ein positiver Imaginärteil der Wellenzahl eine 
Kapitel 3. Modellierung der konvektiven Instabilität
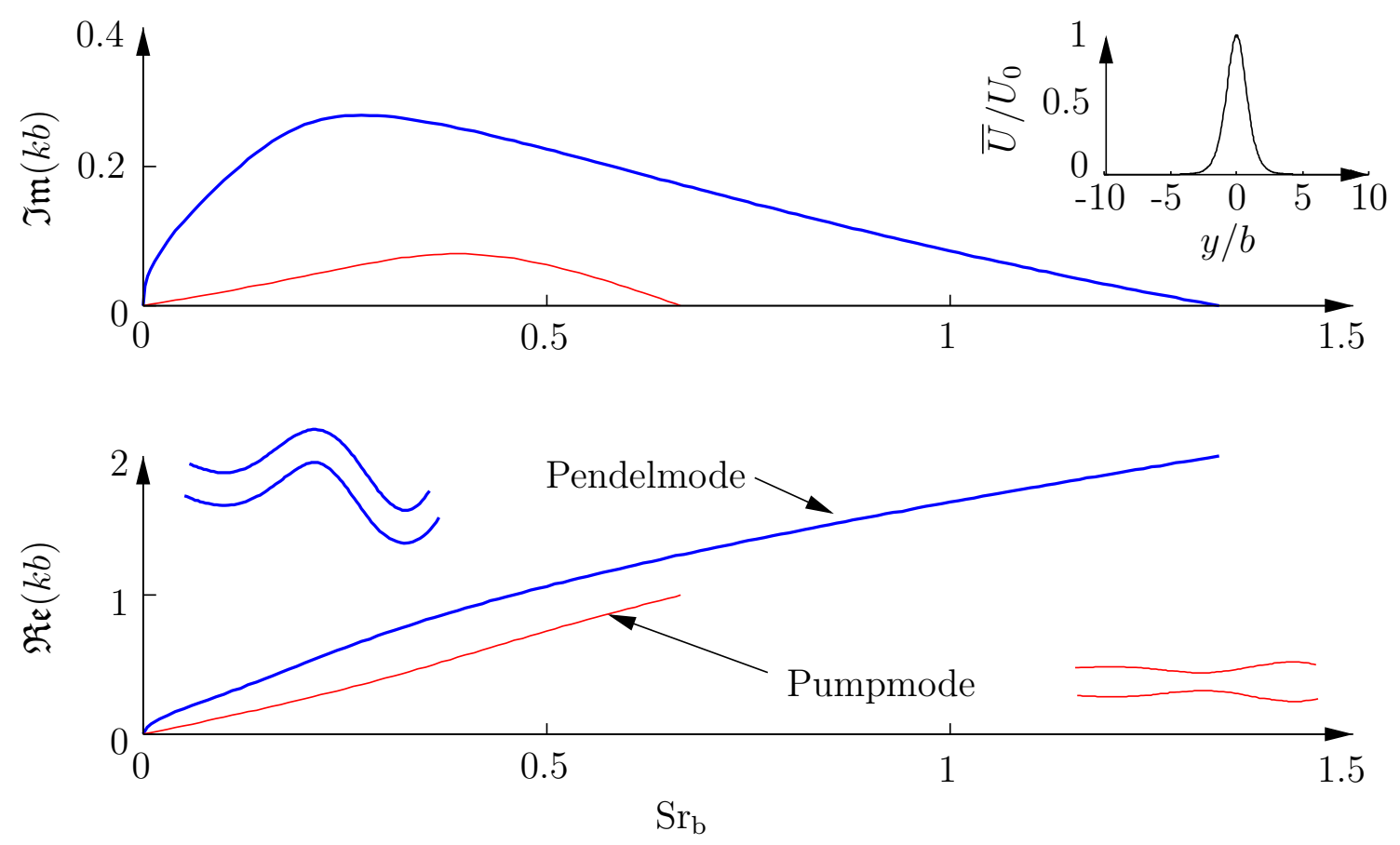

Abb. 3.1: Pump- und Pendelmode für einen Freistrahl mit $\bar{U}(y)=U_{0} / \cosh (y / b)^{2}$-Profil. $\left(S r_{\mathrm{b}}=2 \pi f b / U_{0}\right.$ ist die auf den Skalierungsfaktor $b$ bezogene Strouhalzahl).

Anfachung darstellt. Durch die linearisierte Eulergleichung lässt sich für die Amplitude der Wechselstromfunktion $\hat{\Phi}(y)$ die Rayleigh-Gleichung [41] ableiten:

$$
\left(\bar{U}(y)-\frac{\omega_{\mathrm{s}}}{k}\right)\left(\frac{\partial^{2} \hat{\Phi}}{\partial y^{2}}-k^{2} \hat{\Phi}\right)-\frac{\partial^{2} \bar{U}(y)}{\partial y^{2}} \hat{\Phi}(y)=0,
$$

wobei als Randbedingung zu fordern ist, dass $\hat{\Phi}(y)$ für $y \rightarrow \pm \infty$ verschwindet; das gleiche gilt an den Kanalwänden, da dort die Normalschnelle $\hat{v}=-\frac{\partial \hat{\Phi}}{\partial x}=-i k \hat{\Phi}=0$. Die Lösungen dieser Eigenwertgleichung liefern komplexe Dispersionsrelationen zwischen der eingebrachten reellen Störfrequenz $\omega_{\mathrm{s}}$ und der komplexen Wellenzahl $k\left(\bar{U}, \omega_{\mathrm{s}}\right)$. Oberhalb einer gewissen Grenzfrequenz $\omega_{\text {grenz }}$ findet jedoch keine Wellenausbreitung im Sinne des in Gl. (3.2) gemachten Exponentialansatzes mehr statt, so dass die Dispersionsrelation für $\omega>\omega_{\text {grenz }}$ nicht definiert ist. Die daraus entstehenden Probleme sind in Kap. 3.7 beschrieben.

Analytische Lösungen der Rayleigh-Gleichung 3.3 sind von S.CHANDRASEKHAR [6] zusammengetragen worden. Numerische Methoden sind bei A.Michalke [33], R.Betchow \& W.O. Criminale [2] zu finden. Hier wurde ein Ricatti Schießverfahren mit adaptiver Schrittweitensteuerung [17] zur Lösung der Rayleigh-Gleichung verwendet.

Das gemessene Gleichströmungsprofil ist die Eingangsgröße der Rayleighgleichung, aus der die zu $\omega_{\mathrm{s}}$ gehörige Wellenzahl $k$ berechnet wird. Die Messfehler des Gleichströmungsprofils wirken sich nur dann besonders stark auf $k$ aus, wenn die räumliche Verteilung der Messfehler nach 


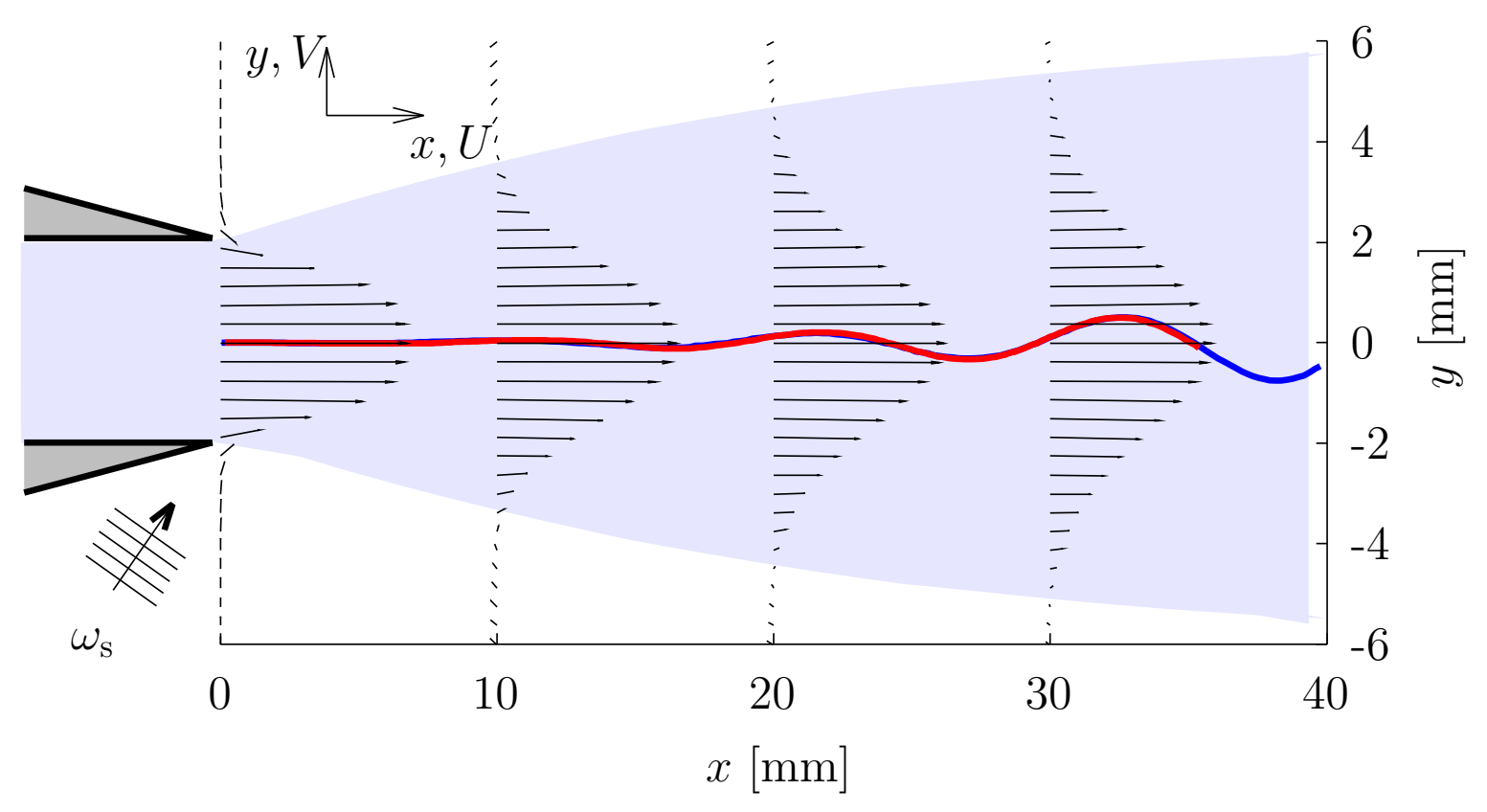

Abb. 3.2: WKB-Methode für leicht divergentes Strömungsprofil. In das Strömungsprofil ist eine mittels WKB-Methode errechnete Streichlinie (blau) und eine gemessene Streichlinie (rot) eingezeichnet (Parameter: $U_{\mathrm{D}}=5.35 \mathrm{~cm} / \mathrm{s},\left|\hat{a}_{\text {Akt. }}\right|=60$ Steps, Störfrequenz $f_{\mathrm{s}}=3 \mathrm{~Hz}$ ).

Raumwellenzahlen zerlegt besonders große Anteile in der Nähe der Wellenzahl $k$ aufweist (siehe Anhang A.1). Die typischerweise kleinräumigen und damit auch verglichen mit $k$ hochwellenzahligen Messfehler haben daher eine relativ geringe Auswirkung auf die Wellenzahl $k$.

Für das Strömungsprofil eines Freistrahls ergeben sich zwei verschiedene Lösungen der RayleighGleichung im Sinne der Stromlinienauslenkung: die antisymmetrische Mode (Pumpmode) und die symmetrische Mode (Pendelmode), die in Abb. 3.1 anhand der zugehörigen Dispersionsrelationen gezeigt sind. Durch die hier verwendeten Aktuatoren, die ein rein symmetrisches Feld erzeugen, kann nur die Pendelmode angeregt werden. Die Keilrückkopplung erzeugt in erster Näherung ein Dipolfeld, so dass auch durch den Keil nur die Pendelmode angeregt werden kann. Die Pumpmode erhielte man dadurch, dass man den Volumenfluss periodisch ändert oder anstelle des Keils eine Prallplatte installiert.

\subsection{WKB-Methode für nahezu parallele Strömungen}

In Abbildung 3.2 ist das stromab breiter werdende Gleichströmungsprofil und das verwendete Koordinatensystem eingezeichnet. Die Lösung der Rayleigh-Gleichung (3.3) für das Profil $\left.\bar{U}(x, y)\right|_{x=\text { const. }}$ liefert zu einer gegebenen reellen Störfrequenz $\omega_{s}$ eine lokale Wellenzahl $k(x)$ und eine lokale Stromfunktion $\hat{\Phi}(y, x)$, wobei für die Lösung der Rayleigh-Gleichung die $x$ Positon nur als Parameter eingeht. Ändert sich das mittlere Strömungsprofil $\bar{U}(x, y)$ nur lang- 
Kapitel 3. Modellierung der konvektiven Instabilität

sam stromab - die Strömung bleibt also nahezu eine Parallelströmung - so kann man die einzelnen Wechselprofile der Stromfunktion $\left.\hat{\Phi}(x, y)\right|_{x=\text { const. }}$ durch eine $x$-abhängige Korrekturfunktion $A_{0} A_{\mathrm{WKB}}(x)$ mit geeignet normierter Anfangsbedingung $A_{\mathrm{WKB}}(x=0)$ aneinander setzen, so dass die Impulsgleichung auch in $x$-Richtung - zumindest in erster Ordnung bezüglich $\frac{\partial U}{\partial x} / U$ - erfüllt wird. Die Darstellbarkeit des Wechselströmungsfeldes

$$
\tilde{\Phi}_{\mathrm{WKB}}(x, y, t)=A_{0} \cdot A_{\mathrm{WKB}}(x) \hat{\Phi}\left(x, y, \omega_{\mathrm{s}}\right) e^{i\left(\omega_{\mathrm{s}} t-\int_{x_{\mathrm{D}}}^{x} k\left(x^{\prime}\right) d x^{\prime}\right)}
$$

als Produkt eines langsam stromab veränderlichen Teils $\hat{\Phi}\left(x, y, \omega_{\mathrm{s}}\right)$ und eines schnell variierenden Teils $\exp \left(-i \int k(x) d x\right)$ ist Voraussetzung für die Anwendtbarkeit der WKB-Methode $([18,16])$. D.h., die WKB-Methode ist eine gute Näherung, wenn die Welle lokal ein paralleles Geschwindigkeitsfeld sieht, oder anders ausgedrückt, wenn sich die Skalen auf dem sich die beiden Faktoren von $\tilde{\Phi}_{\mathrm{WKB}}$ ändern, möglichst stark voneinander unterscheiden, also

$$
\left|\frac{\partial\left(\ln \hat{\Phi}_{\mathrm{WKB}}\right) / \partial x}{k}\right| \propto \frac{1}{U_{0}(x)} \frac{\partial U_{0}(x)}{\partial x} /|k(x)|=: \epsilon
$$

möglichst klein ist, wobei $U_{0}(x)$ die Strahlmittengeschwindigkeit ist.

Mit zunehmender Strouhalzahl $\mathrm{Sr}_{\mathrm{d}}=\omega_{\mathrm{S}} \mathrm{d} / \mathrm{U}_{\mathrm{D}}$ ist dies immer besser erfüllt. Setzt man nun den Ansatz von Gleichung 3.4 in die Euler-Gleichung ein und behält nur die Terme der Größenordnung $\epsilon$ bei, so ergibt sich nach Mittelung über $y$ gemäß des Skalarprodukts $\int\{\cdot\} \hat{\Phi}^{*} d y$ :

$$
M(x) \frac{d A_{\mathrm{WKB}}(x)}{d x}+N(x) A_{\mathrm{WKB}}(x)=0,
$$

wobei

$$
\begin{aligned}
M(x)= & \int_{-\infty}^{\infty}\left\{2 k \omega_{\mathrm{s}} \hat{\Phi}+\bar{U}\left(\hat{\Phi}^{\prime \prime}-3 k^{2} \hat{\Phi}\right)-\bar{U}^{\prime \prime} \hat{\Phi}\right\} \hat{\Phi}^{*} d y \\
N(x)= & \int_{-\infty}^{\infty}\left\{\hat{\Phi}^{\prime} \frac{\partial \bar{U}^{\prime}}{\partial x}+\bar{V}\left(\hat{\Phi}^{\prime \prime \prime}-k^{2} \hat{\Phi}^{\prime}\right)-\bar{U}^{\prime \prime} \frac{\partial \hat{\Phi}}{\partial x} \cdots\right. \\
& \left.+U\left(\frac{\partial \hat{\Phi}^{\prime \prime}}{\partial x}-3 k^{2} \frac{\partial \hat{\Phi}}{\partial x}-3 k \hat{\Phi} \frac{d k}{d x}\right)+\omega_{\mathrm{s}}\left(\hat{\Phi} \frac{d k}{d x}+2 k \frac{\partial \hat{\Phi}}{\partial x}\right)\right\} \hat{\Phi}^{*} d y
\end{aligned}
$$

(dabei ist ' die Ableitung $\frac{\partial}{\partial y}$, und komplex konjugiert wird mit * gekennzeichnet). Durch die Lösung dieser Gleichung gewinnt man insgesamt aus dem Gleichströmungsprofil $\bar{U}(x, y)$ eine Lösung für die Instabilitätswelle, die nur noch einen freien Parameter hat, nämlich die Amplitude des Instabilitätswellenwechselfeldes $A_{0}$.

\subsection{Parametrisierung des Gleichströmungsprofils}

Zur Ermittlung des Strömungsprofils des ebenen Freistrahls wurden von PRECKEL 1999 [39] Messungen mittels Laser-Doppler-Anemometrie durchgeführt. Die gemessenen Strömungsgeschwindigkeiten können in guter Näherung durch Strömungsprofile beschrieben werden, die aus 


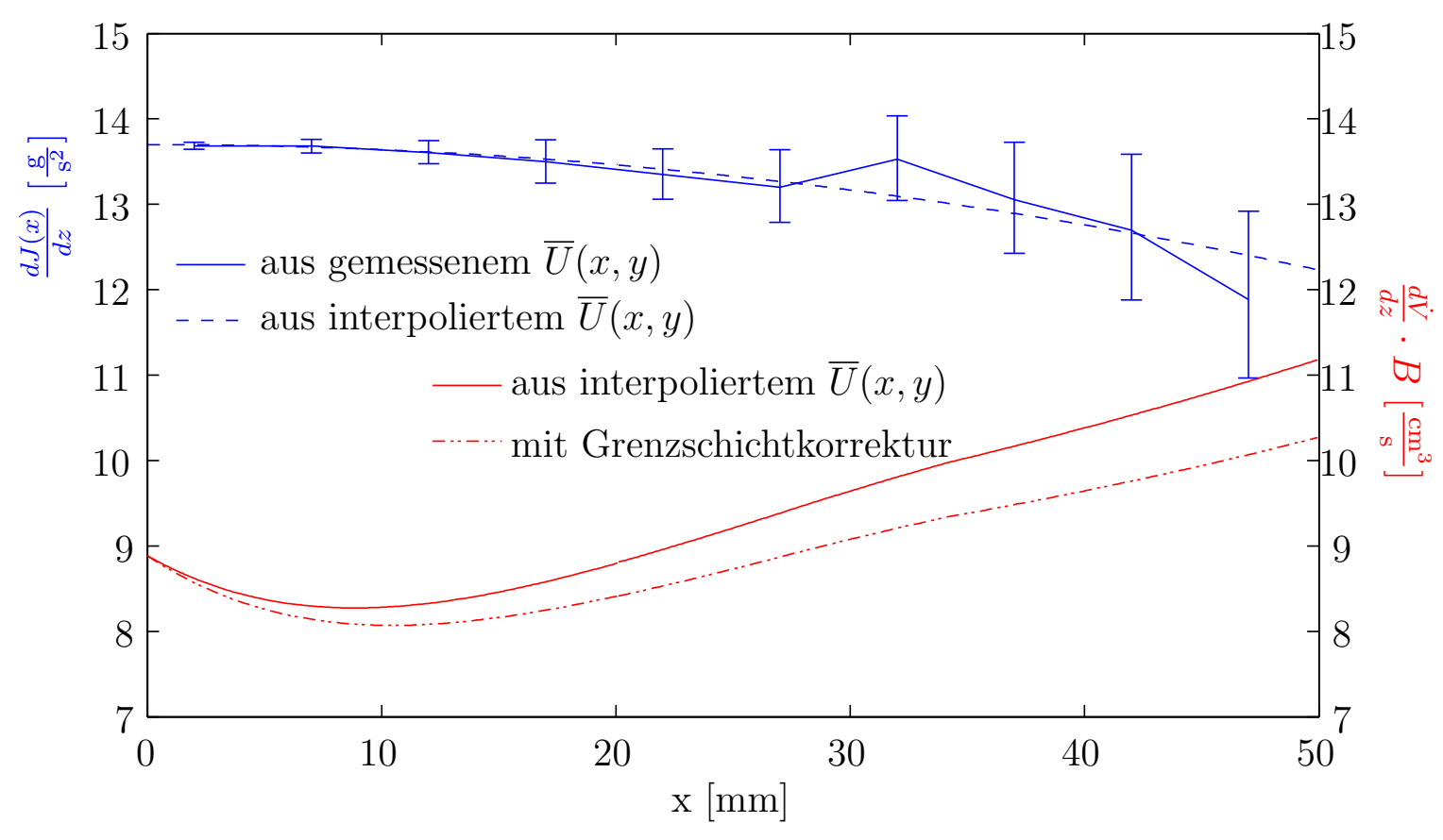

Abb. 3.3: Impulsfluss und Volumenfluss. Der Volumenfluss ist auf die Kanaltiefe $B=$ $40 \mathrm{~mm}$ bezogen. Berücksichtigt man noch das Anwachsen der Grenzschichten in $z$-Richtung, so ergibt sich der dargestellte korrigierte Volumenfluss.

zwei gegeneinander verschobenen tanh-Funktionen zusammengesetzt sind:

$$
\bar{U}(x, y)=\frac{U_{0}-U_{\text {ausgl. }}}{2 \tanh (a / 2)} \cdot\left[\tanh \left(\frac{\left(y-y_{0}\right)}{b}+a / 2\right)-\tanh \left(\frac{\left(y-y_{0}\right)}{b}-a / 2\right)\right]+U_{\text {ausgl. }},
$$

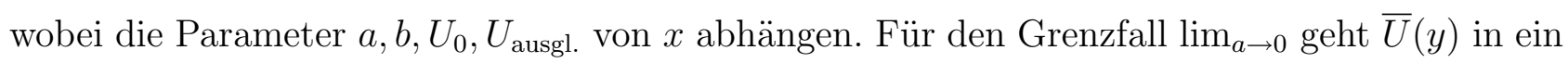
$\cosh ^{-2}(y)$-Profil über.

Um ein für die WKB-Methode höher aufgelöstes interpoliertes Profil $\bar{U}(x, y)$ aus den Geschwindigkeitsdaten herzustellen, wurden die integralen Größen $\frac{\partial J(x)}{\partial z}$

$$
\frac{d J(x)}{d z}=\rho \int_{-y_{0}}^{y_{0}} \bar{U}(x, y)^{2} d y
$$

in Anlehnung an den Impulsfluss und $\frac{\partial \dot{V}}{\partial z}$

$$
\frac{d \dot{V}(x)}{d z}=\int_{-y_{0}}^{y_{0}} \bar{U}(x, y) d y
$$

in Anlehnung an den Volumenfluss durch glatte Funktionen angepasst (siehe Abb. 3.3). Um den tatsächlichen Impulsfluss und Volumenfluss zu ermitteln, müssen die Größen noch in Spannweitenrichtung $z$ über die Kanaltiefe $B$ integriert werden und $y_{0}$ bis zu den seitlichen Kanalwänden 


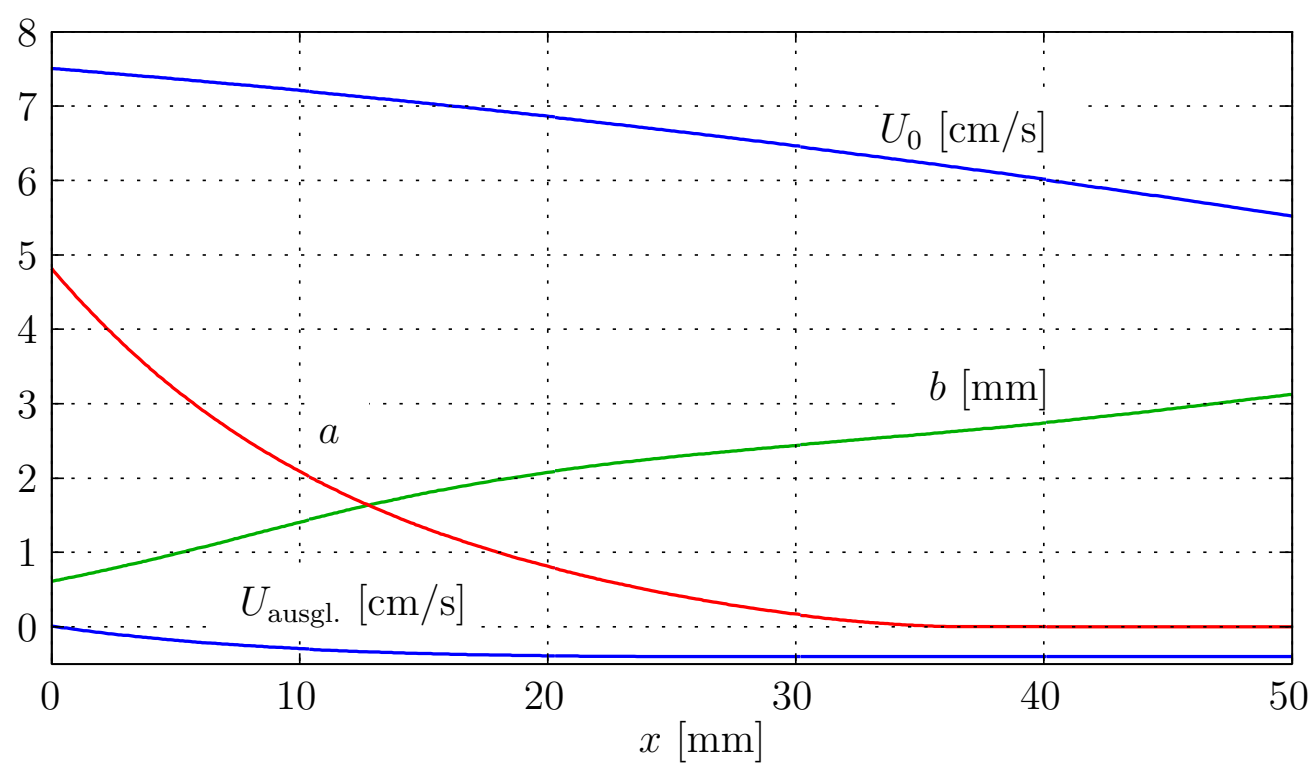

Abb. 3.4: Parameter der Profilentwicklung.

ausgedehnt werden. Man erwartet durch die Messung in der Kanalmitte einen Anstieg des Volumenflusses, da auf Grund der Verengung des Kanals durch die seitlich wachsenden Grenzschichten die Strömungsgeschwindigkeit in der Kanalmitte steigt. Allerdings reicht die Zunahme der Grenzschichtdicke, die man aus der ebenen Platte mit der Anströmgeschwindigkeit $U_{\mathrm{D}}$ erhält, nicht aus, um die Erhöhung des Volumenflusses in der Strahlmitte zu erklären (siehe gestrichelte rote Kurve in Abb. 3.3).

Durch die Mitnahme des ruhenden Mediums am Strahlrand etabliert sich eine Ausgleichströmung, die das Medium von stromab in Richtung Düse transportiert. Dieses stromauf fließende Medium muss durch einen negativen Druckgradienten stromab beschleunigt werden, daher nimmt der Druck mit dem Laufweg ab und führt zu einem Anstieg des Impulsflusses. Die in Abb. 3.3 gezeigte leichte Abnahme des Impulsflusses mit zunehmendem Laufweg ist daher unerwartet. Allerdings ist der Freistrahl ohne Kühlung aufgrund der Pumpen etwas wärmer als das ihn umgebende Medium. Der ganze Strahl erfährt daher einen Auftrieb, der seine Geschwindigkeit verlangsamt. Das schwerere, den Strahl umgebende Medium sinkt zu Boden und verlangsamt damit die Ausgleichsströmung, womit der Impulsfluss abnimmt. Ist die Freistrahltemperatur $T_{0}+\Delta T$ und die Temperatur des umgebenden Mediums $T_{0}$, so ergibt sich durch die Auftriebskräfte ein Druckgradient der Größe

$$
\frac{\partial p_{\text {Auftrieb }}}{\partial x}=-\frac{d \rho}{d T} \cdot \Delta T \cdot g
$$

mit Erdbeschleunigung $g$. Vergleicht man die Gesamtdruckdifferenz über die Länge des hier betrachteten Strahlabschnitts $(x \leq L=5 \mathrm{~cm})$

$$
p_{\text {Auftrieb }}=-\frac{d \rho}{d T} \cdot \Delta T g L
$$


mit dem Staudruck $p_{\text {Stau }}=1 / 2 \rho U_{\mathrm{D}}^{2}$, so ergibt sich als Druckverhältnis

$$
\frac{p_{\text {Auftrieb }}}{p_{\text {Stau }}}=\Delta T \frac{d \rho}{d T} \frac{2 L g}{\rho U_{\mathrm{D}}^{2}}=0.10
$$

wenn man $3 \mathrm{~K}$ Temperaturdifferenz bei $22^{\circ} \mathrm{C}$ und $\frac{d \rho}{d T} / \rho \approx 10^{-4} \frac{\mathrm{g}}{\mathrm{cm}^{3} \mathrm{~K}}$ und $U_{\mathrm{D}}=5.36 \mathrm{~cm} / \mathrm{s}$ annimmt. In dieser Größenordnung liegt auch die Abnahme des Impulsflusses in Abb. 3.3. Mit den diskutierten Größen $\frac{\partial J}{\partial z}$ und $\frac{\partial \dot{V}}{\partial z}$ können nun die das Profil beschreibenden Parame-

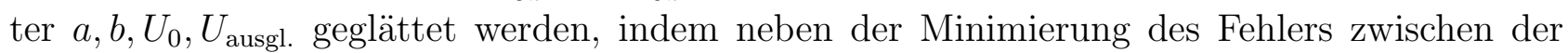
anzupassenden Funktionenschar Gl. (3.7) und den Geschwindigkeitsrohdaten auch die vorgegebenen geglätteten Größen $\frac{d J(x)}{d z}$ und $\frac{d \dot{V}(x)}{d z}$ angenähert werden. Daraus erhält man eine glatte Parameterschar, wie sie in Abb. $3.4 \mathrm{zu}$ sehen ist.

\subsection{Anfachung und Phase des instationären Strömungs- felds und der Streichlinien}

Durch die WKB-Methode kann das Wechselfeld der Instabilitätswelle

$$
\underline{\tilde{u}}_{\mathrm{WKB}}=\left(\tilde{u}_{\mathrm{WKB}}, \tilde{v}_{\mathrm{WKB}}\right)=\left(\frac{\partial \tilde{\Phi}_{\mathrm{WKB}}}{\partial y},-\frac{\partial \tilde{\Phi}_{\mathrm{WKB}}}{\partial x}\right)
$$

bestimmt werden.

Da der Anregungsmechanismus der Instabilitätswelle nicht bekannt ist, der das Wechselfeld $\underline{\tilde{u}}_{\mathrm{WKB}}$ und die im Kanal vorhandenen Instabilitätswelle $\underline{\tilde{u}}_{\text {Inst. }}$ in Beziehung setzt, wird als Normierungsgröße für die Wechselgeschwindigkeit $v_{\text {Akt. }}^{\mathrm{n}}$ eingeführt, die durch das Maximum des anregenden Geschwindigkeitsfeldes in der Kanalmitte $\tilde{v}(x, y \equiv 0)_{\text {Akt. }}$ gegeben ist:

$$
v_{\mathrm{Akt} .}^{\mathrm{n}}=\max _{x}\left|\hat{v}_{\mathrm{Akt}}(x, 0)\right|
$$

Mit dem Gleichströmungsprofil $\underline{\bar{U}}=(\bar{U}, \bar{V})=\left(\frac{\partial \bar{\Phi}}{\partial y}, \frac{\partial \bar{\Phi}}{\partial x}\right)$ kann das Geschwindigkeitsfeld als

$$
\underline{U}(x, y, t)=\underline{\bar{U}}+A_{0} \cdot \underline{\tilde{u}}_{\mathrm{WKB}} / v_{\mathrm{Akt}}^{\mathrm{n}} \cdot\left|v_{\mathrm{Akt} .}^{\mathrm{n}} / \hat{a}_{\mathrm{Akt} .}^{0}\right| \cdot\left|\hat{a}_{\mathrm{Akt} .}\right|=\underline{\bar{U}}+A_{0} \cdot\left|\hat{a}_{\mathrm{Akt} .}\right| \cdot \underline{\tilde{u}}_{\mathrm{WKB}}^{0}
$$

dargestellt werden, dabei ist $\left|v_{\text {Akt. }}^{\mathrm{n}} / \hat{a}_{\text {Akt. }}^{0}\right|$ die Geschwindigkeit, die eine Aktuatoreinheit - also ein Step - erzeugt, und $A_{0}$ gibt die Effektivität der Instabilitätswellenanregung an. Damit ist das normierte Geschwindigkeitswechselfeld $\underline{\tilde{u}}_{\mathrm{WKB}}^{0}$ unabhängig von der Aktuatoramplitude.

Als Normierungsgröße für die Streichlinienauslenkung $\eta$ dient entsprechend die Potenzialfeldauslenkung $\zeta_{\text {Akt. }}^{\mathrm{n}}$ am Ort $x_{\max }$, wie in Anhang C.1 näher erläutert ist.

Im Folgenden soll das unterschiedliche Anwachs- und Phasenverhalten der lateralen Wechselgeschwindigkeit $\tilde{v}_{\text {WKB }}$ in Kanalmitte und der Streichlinienauslenkung $\tilde{\eta}$ als Funktion des Düsenabstandes untersucht werden. 
Kapitel 3. Modellierung der konvektiven Instabilität

Bildet man den komplexen Gradienten $i \nabla_{x} \ln (\cdot)$ der entsprechenden fouriertransformierten Größe, so wird das Anwachsverhalten durch die Anfachung $\mathfrak{I m}\left\{i \nabla_{x} \ln (\cdot)\right\}$ mit dem Laufweg charakterisiert und die Phasendrehung pro Lauflänge durch den negativen Phasengradienten $\mathfrak{R e}\left\{i \nabla_{x} \ln (\cdot)\right\}=-\frac{\partial \arg (\cdot)}{\partial x}$.

\section{Vergleich zwischen korrigierter gemessener und durch das WKB-Wechselfeld inte- grierter Streichlinie}

Die Streichlinie $\tilde{\eta}_{\text {WKB }}$ kann nun durch das Geschwindigkeitsfeld

$$
\underline{U}(x, y, t)=\underline{\bar{U}}+A_{0} \cdot \underline{\tilde{u}}_{\mathrm{WKB}}=A_{0}\left|\hat{a}_{\mathrm{Akt} .}\right| \cdot \underline{\tilde{u}}_{\mathrm{WKB}}^{0}
$$

numerisch integriert werden. Die Startposition $\left(x_{\text {start }}, y_{\text {start }} \equiv 0\right)$ der Teilchen, die die Streichlinie markieren, muss deutlich stromauf liegen, so dass die Auslenkung des Wechselfelds am Ort $x_{\text {start }}$ klein gegen die am Ort $x=0$ ist. So werden die Teilchen näherungsweise in dieselbe Stromröhre injiziert und die Streichlinie hängt nicht mehr wesentlich von $x_{\text {start }}$ ab. Um dies zu erreichen wurde $\underline{\tilde{u}}_{\mathrm{WKB}}^{0}$ bis zu $x=-2 d=8 \mathrm{~mm}$ stromauf extrapoliert, wobei für $x \leq 0$ die Profilparameter von $x=0$ verwendet wurden. Die Annahme, dass durch die Extrapolation der Instabilitätswelle in die Düse hinein die Streichlinie beschrieben wird, kann gerechtfertigt werden, da die Vorgeschichte der Streichlinie für den hier betrachteten komplexen Gradienten unwesentlich wird, wenn das Geschwindigkeitswechselfeld etwa exponentiell anwächst.

Die Instabilitätswellenamplitude $A_{0}$ wurde so angepasst, dass die numerische Streichlinie und die gemessene Streichlinie (ohne den Anteil des Potenzialfeldes (siehe unten)) im Fernfeld etwa gleich ausgelenkt sind. Weiter zur Düse hin ist das anregende Potenzialfeld, das der Instabilitätswelle überlagert ist, groß im Vergleich zur Instabilitätswelle. Die Wahl von $A_{0}$ wird aufgrund der nichtlinearen Abhängigkeit der Streichlinienauslenkung von der Aktuatoramplitude für kleine Aktuatoramplituden festgelegt.

Die gemessene Streichlinie entsteht durch Integration über ein Geschwindigkeitsfeld, das näherungsweise in die Komponenten $\underline{U}(x, y, t)=\underline{\bar{U}}+\underline{\tilde{u}}_{\text {Inst. }}+\underline{\tilde{u}}_{\text {Akt. }}$ zerlegt werden kann, wobei $\underline{\tilde{u}}_{\text {Inst. }}$ das Wechselfeld der tatsächlichen Instabilitätswelle ist. Die Streichlinienintegration führt im Allgemeinen zusätzlich zu einer nichtlinearen Vermischung der Felder $\underline{\tilde{u}}_{\text {Inst. }}$ und $\underline{\tilde{u}}_{\text {Akt. }}$, so dass die Auftrennung

$$
\tilde{\eta}=\tilde{\eta}_{\text {Inst. }}+\tilde{\eta}_{\text {Akt. }}
$$

in einen Anteil $\tilde{\eta}_{\text {Akt. }}$ aufgrund des anregenden Aktuatorfeldes $\underline{\tilde{u}}_{\text {Akt. }}$ und in einen Anteil $\tilde{\eta}_{\text {Inst. }}$ aufgrund des Instabilitätswellenfeldes $\underline{\tilde{u}}_{\text {Inst. }}$ nur näherungsweise und durch Rückrechnung geschehen kann (siehe Anhang C.4). Ohne die Bereinigung von $\tilde{\eta}$ um den durch $\underline{\tilde{u}}_{\text {Akt. }}$ entstandenen Anteil, wäre ein sinnvoller Vergleich anhand des komplexen Gradienten nicht möglich, da die Interferenz von $\tilde{\eta}_{\text {Inst. }}$ mit $\tilde{\eta}_{\text {Akt. }}$ zu großen Schwankungen führt. Ein kleiner Anteil der Interferenz bleibt jedoch im düsennahen Bereich bestehen (siehe Abb. 3.5), der sich dort in der Welligkeit (siehe Anhang C.4) des komplexen Gradienten äußert. 

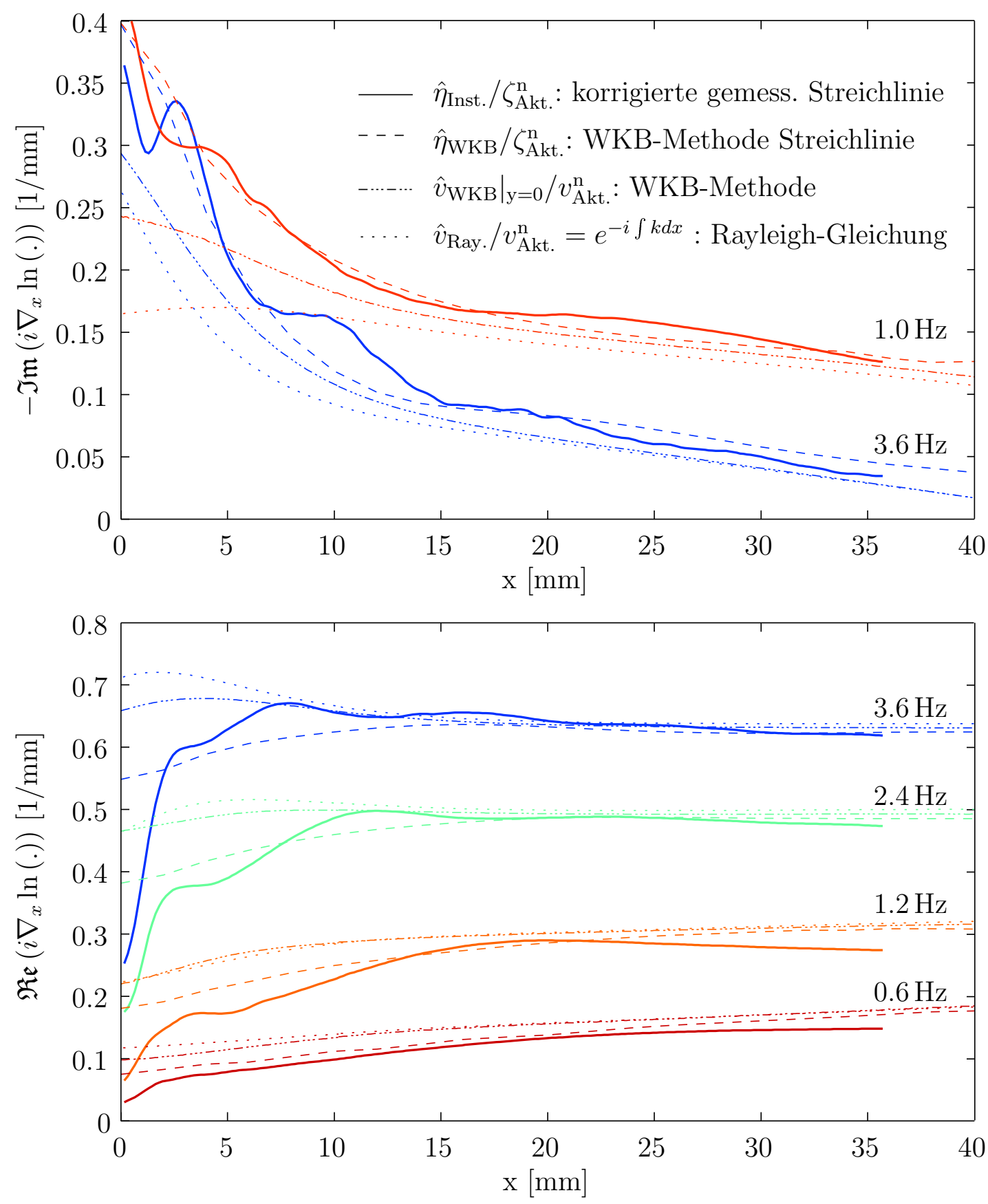

Abb. 3.5: Komplexer Phasengradient für verschiedene Methoden und Größen bei verschiedenen reellen Anregungsfrequenzen. Vergleich zwischen der linearen Theorie der RayleighGleichung, der Wechselgeschwindigkeit $\left.\hat{v}_{\mathrm{WKB}}(x, y)\right|_{\mathrm{y}=0}$ aus der WKB-Methode, der aus dem WKB-Wechselfeld integrierten Streichlinie $\hat{\eta}_{\mathrm{WKB}}$ und der die Instabilitätswelle charakterisierenden korrigierten gemessenen Streichlinie $\hat{\eta}_{\text {Inst. }}$ 


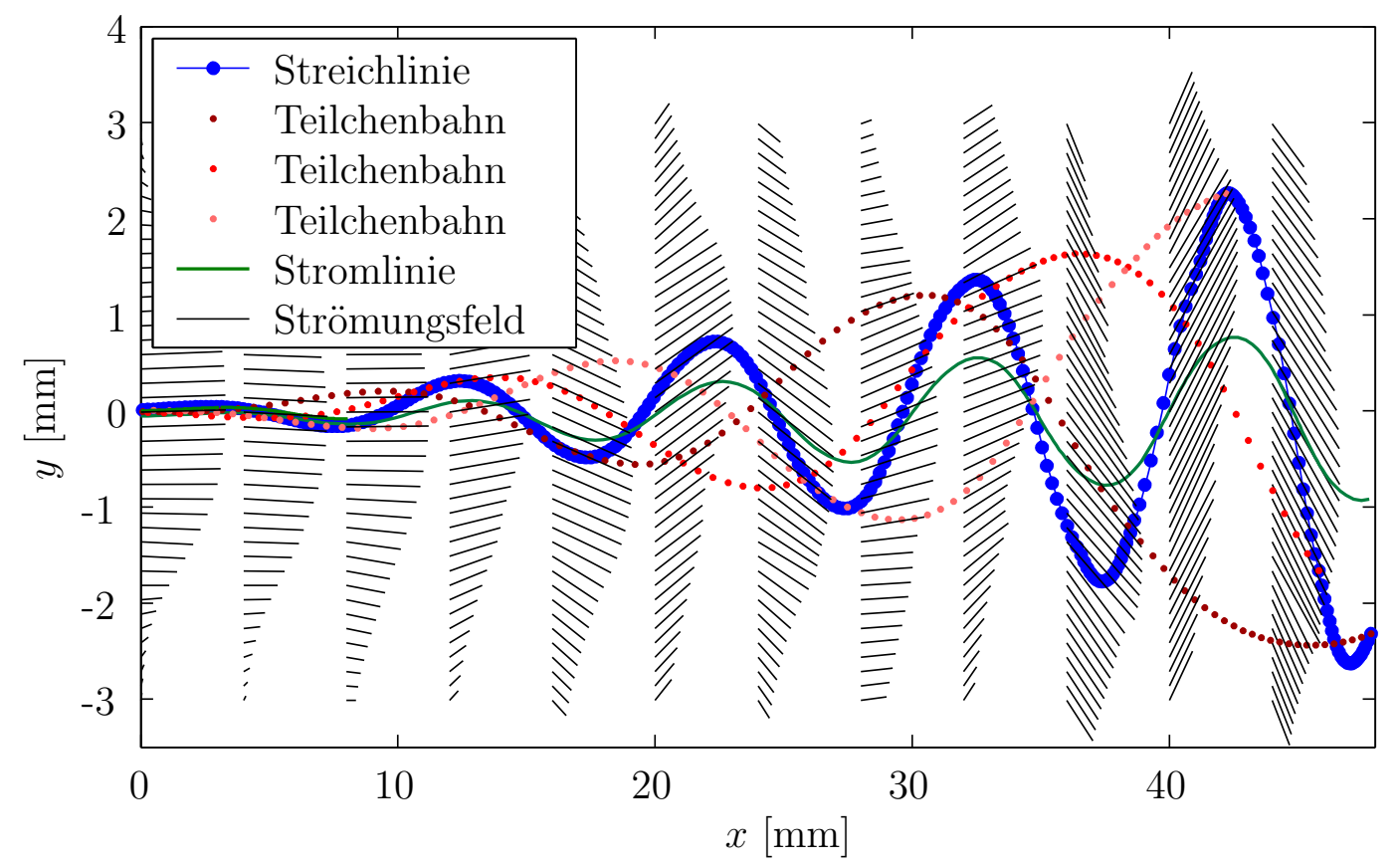

Abb. 3.6: Momentanes Strömungsfeld zusammen mit der Stromlinie und der integrierten Streichlinie. Zusätzlich sind drei Teilchenbahnen eingezeichnet, wobei der zeitliche Abstand der Punkte $\left(1 / 30 T_{\mathrm{s}}\right)$ ist. Parameter: $f_{\mathrm{s}}=3.6 \mathrm{~Hz},\left|\hat{a}_{\mathrm{Akt}}\right|=300$ Steps.

Der lokale komplexe Gradient $i \nabla_{x} \ln \left(\hat{\eta}_{\text {Inst. }}(x) / \zeta_{\text {Akt. }}^{\mathrm{n}}\right)$ der experimentell gemessenen Streichlinienauslenkung kann nun mit dem theoretischen $i \nabla_{x} \ln \left(\tilde{\eta}_{\mathrm{WKB}}(x) / \zeta_{\text {Akt. }}^{\mathrm{n}}\right)$ der WKB-Streichlinie verglichen werden. Die theoretische Anfachung - der Imaginärteil des komplexen Gradienten - der WKB-Streichlinie $\hat{\eta}_{\mathrm{WKB}}$ nähert die der experimentellen Streichlinie $\hat{\eta}_{\text {Inst. }}$ sehr gut an. Die experimentellen Streichlinien sind mit kleinen Aktuatorauslenkungen (50 Steps) gemessen. Geht man zu größeren Anregungsamplituden über, so wird die Streichlinie sehr schnell nichtlinear verzerrt (siehe Abschnitt 3.5), und für sehr große Amplituden beginnt die Streichlinie sich aufzurollen (siehe Abb. 3.7).

\section{Vergleich des Wechselgeschwindigkeitsfelds mit der integrierten Streichlinie}

Der für die Untersuchung wichtige ortsabhängige Zusammenhang zwischen Instabilitätswellenfeld $\hat{v}_{\mathrm{WKB}}^{0}$ und Streichlinienauslenkung $\tilde{\eta}_{\mathrm{WKB}}$ spiegelt sich im Unterschied der entsprechenden Wellenzahlen $i \nabla_{x} \ln \left(\hat{v}_{\mathrm{WKB}} / v_{\text {Akt. }}^{\mathrm{n}}.\right)$ und $i \nabla_{x} \ln \left(\hat{\eta}_{\mathrm{WKB}} / \zeta_{\text {Akt. }}^{\mathrm{n}}.\right)$ wider, der in Abb. 3.5 gezeigt ist. Die Anfachung der Streichlinie ist etwas größer, als die des WKB-Wechselfeldes $\left.\hat{v}_{\mathrm{WKB}}^{0}(x, y)\right|_{\mathrm{y}=0}$.

Betrachtet man den Realteil des komplexen Gradienten, so ist im düsennahen Bereich $\mathfrak{R e}\left(i \nabla_{x} \ln \left(\hat{\eta}_{\mathrm{WKB}} / \zeta_{\text {Akt. }}^{\mathrm{n}}\right)\right)$ um etwa $1 / 4$ kleiner als $\mathfrak{R e}\left(i \nabla_{x} \ln \left(\hat{v}_{\mathrm{WKB}} / v_{\text {Akt. }}^{\mathrm{n}}\right)\right)$. Dort ändert sich das Profil noch stark als Funktion von $x$ und die WKB-Bedingung: $\epsilon \ll 1$ (siehe Gl. (3.5)) ist besonders für tiefe Frequenzen immer weniger erfüllt, jedoch ist die Übereinstimmung mit den Mess- 
ergebnissen sehr gut. Mit zunehmendem Düsenabstand werden die Abweichungen zwischen den komplexen Gradienten der Streichlinie und denen des Wechselfeldes schnell kleiner. MAUREL [27] hat durch die experimentell bestimmte Ausbreitungsgeschwindigkeit der Instabilitätswelle im Verhältnis zu der des Hauptströmungsprofils abgeschätzt, dass die „Phasengeschwindigkeit“ (siehe Abschnitt 3.6) der Streichlinie um circa 7,5\% größer ist als die des Wechselfeldes $\hat{v}^{0}$ $\left(\left|\mathfrak{R e}\left(i \nabla_{x} \ln \left(\hat{\eta} / \zeta_{\text {Akt. }}^{\mathrm{n}}\right)\right)\right|<\left|\mathfrak{R e}\left(i \nabla_{x} \ln \left(\hat{v} / v_{\text {Akt. }}^{\mathrm{n}}\right)\right)\right|\right)$, was man hier größenordnungsmäßig bestätigt sieht.

In Abb. 3.6 ist der wohlbekannte Unterschied zwischen Bahnlinien, Stromlinien und Streichlinien noch einmal dargestellt. Man erkennt hier vor allem im düsennahen Bereich (vergl. die Maxima), dass die Wellenlänge der Streichlinie etwas größer ist, als die der Stromlinie.

\subsection{Nichtlinearität der Freistrahlinstabilität bezogen auf die Streichlinienauslenkung}

Zwei wesentlich verschiedene Nichtlinearitäten treten bei der Freistrahlinstabilität auf:

- Das Strömungsfeld und die gemessene Streichlinie sind nichtlinear miteinander verknüpft (Nichtlinearität der Messung).

- Die Nichtlinearität des konvektiven Terms der Eulergleichung führt zu einer Rückwirkung der Instabilitätswelle auf das Gleichströmungsprofil. Die durch die Instabilitätswelle entstehende Schubspannung führt zu einer Verbreiterung des Gleichströmungsprofils. Die Anfachung der Freistrahlinstabilität ist daher durch die Instabilitätswelle selbst nichtlinear begrenzt. Dieses Sättigungsverhalten ist analytisch nur schwer zu beschreiben (Nichtlinearität der Wellenausbreitung).

Da beide Prozesse eine ähnliche Abhängigkeit von der Anregungsamplitude aufweisen, ist eine Trennung der beiden unterschiedlichen Nichtlinearitäten kaum möglich. Man müsste alle nichtlinearen Glieder in den maßgeblichen Gleichungen gegeneinander abschätzen. Besonders die Abschätzung der Verbreiterung des Haupströmungsprofils mittels der Reynoldschen Schubspannungen ist problematisch, da die zugrunde liegenden Gleichungen und Ansätze schon linearisiert sind und durch die Vernachlässigung der Viskosität die Gleichungen vor allem in der Nähe der kritischen Schicht die Strömung nicht mehr angemessen beschreiben. Dennoch ist der Versuch einer Beschreibung der Amplitudenabhängigkeit der Freistrahlinstabilität unternommen. Dabei stellt sich heraus, dass beide Prozesse - Wellenausbreitung und Messung der Streichlinie - gegenläufige Einflüsse auf den Betrag des Phasengradienten der Instabilitätswelle haben. Der an einem Ort gemessene Betrag des Phasengradienten nimmt für die Wechselgeschwindigkeit mit wachsender Anregungsamplitude zu, d.h., die Phasengeschwindigkeit der Instabilitätswelle sinkt, während der Betrag des Phasengradienten der Streichlinie mit zunehmender Anregungsamplitude sogar abnimmt und so den ersten Effekt überkompensiert. Bei 

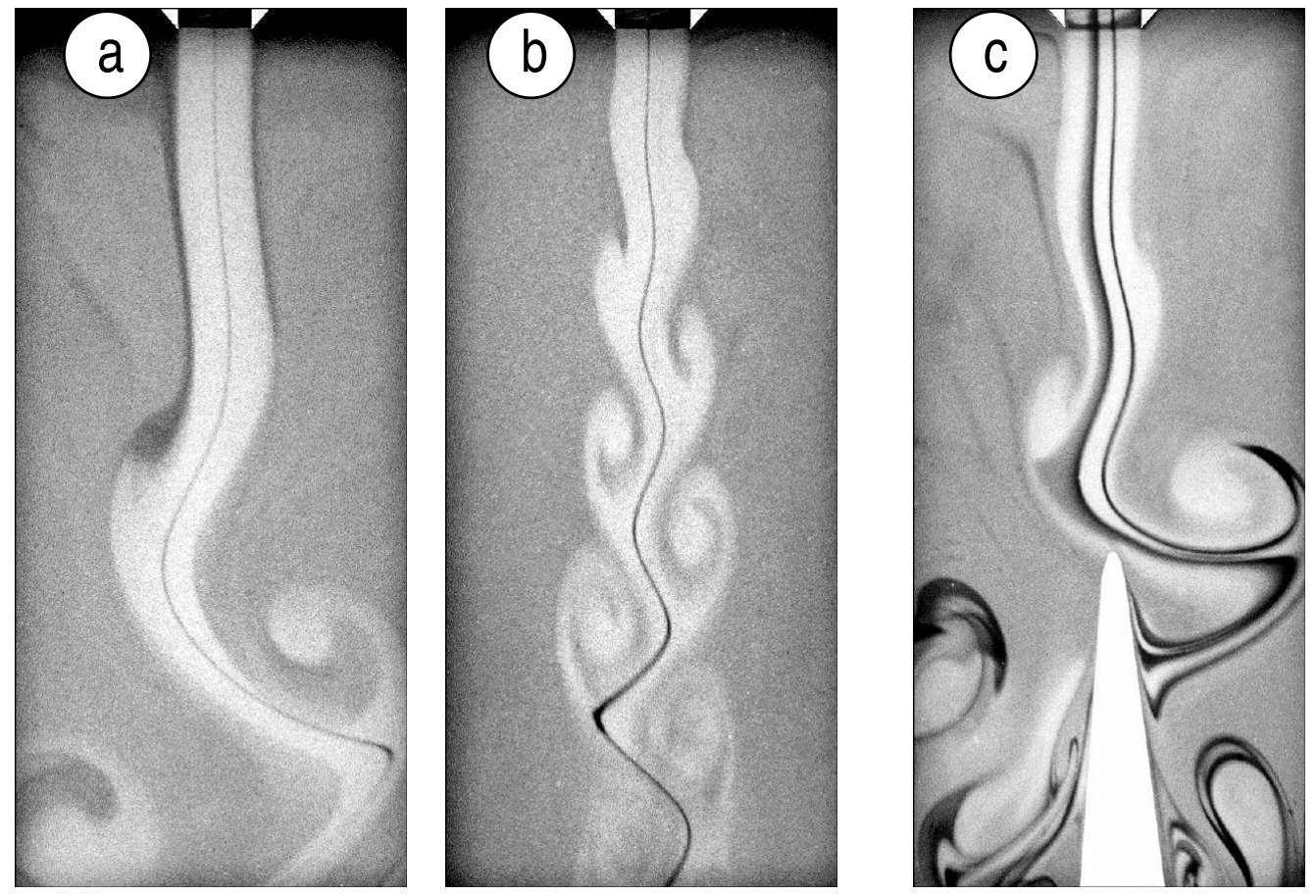

Abb. 3.7: Konvektive Instabilität des Freistrahls. $U_{\mathrm{D}}=5.3 \mathrm{~cm} / \mathrm{s}$ : (a) und (b) mit künstlicher Anregung $f_{\mathrm{s}}=1 \mathrm{~Hz}(\mathrm{a})$ und $f_{\mathrm{s}}=2 \mathrm{~Hz}(\mathrm{~b})$, selbsterregte Schwingung des Strahl-KantenSystems (c).

der Streichlinie sollte man nicht von einer Wellenausbreitung sprechen, daher bleibt der Begriff der Phasengeschwindigkeit dem unterliegenden Wechselfeld vorbehalten. Bei künstlicher Verbreiterung des mittleren Strömungsprofils durch ein eingestreutes breitbandiges Rauschen, beobachtet man tatsächlich, dass die Eigenfrequenz der selbsterregten Schwingung leicht sinkt, was am einfachsten durch eine verminderte Ausbreitungsgeschwindigkeit der Instabilitätswelle beschreibbar ist.

\subsubsection{Sättigungsverhalten der Freistrahlinstabilität}

Der Energiefluss von der Gleichströmung in die angefachte Wechselströmung führt zu einer Veränderung des Gleichströmungsprofils, so dass die Instabilitätswellenanfachung ab einer bestimmten Verbreiterung des Profils mit zunehmender Instabilitätswellenamplitude sinkt. Diese nichtlineare Amplitudenentwicklung soll dadurch beschrieben werden, dass die Instabilitätswelle selbst auf das Profil zurückkoppelt und es verbreitert. Dabei wird die Annahme gemacht, dass zur Beschreibung der Wellenausbreitung die linearisierte Eulergleichung zusammen mit dem zeitlich gemittelten Hauptströmungsprofil ausreicht. Denkt man sich das Strömungsfeld des Strahls (hellere Bereiche und deren Zwischenräume in Abb. 3.7) gemittelt, so erkennt man 


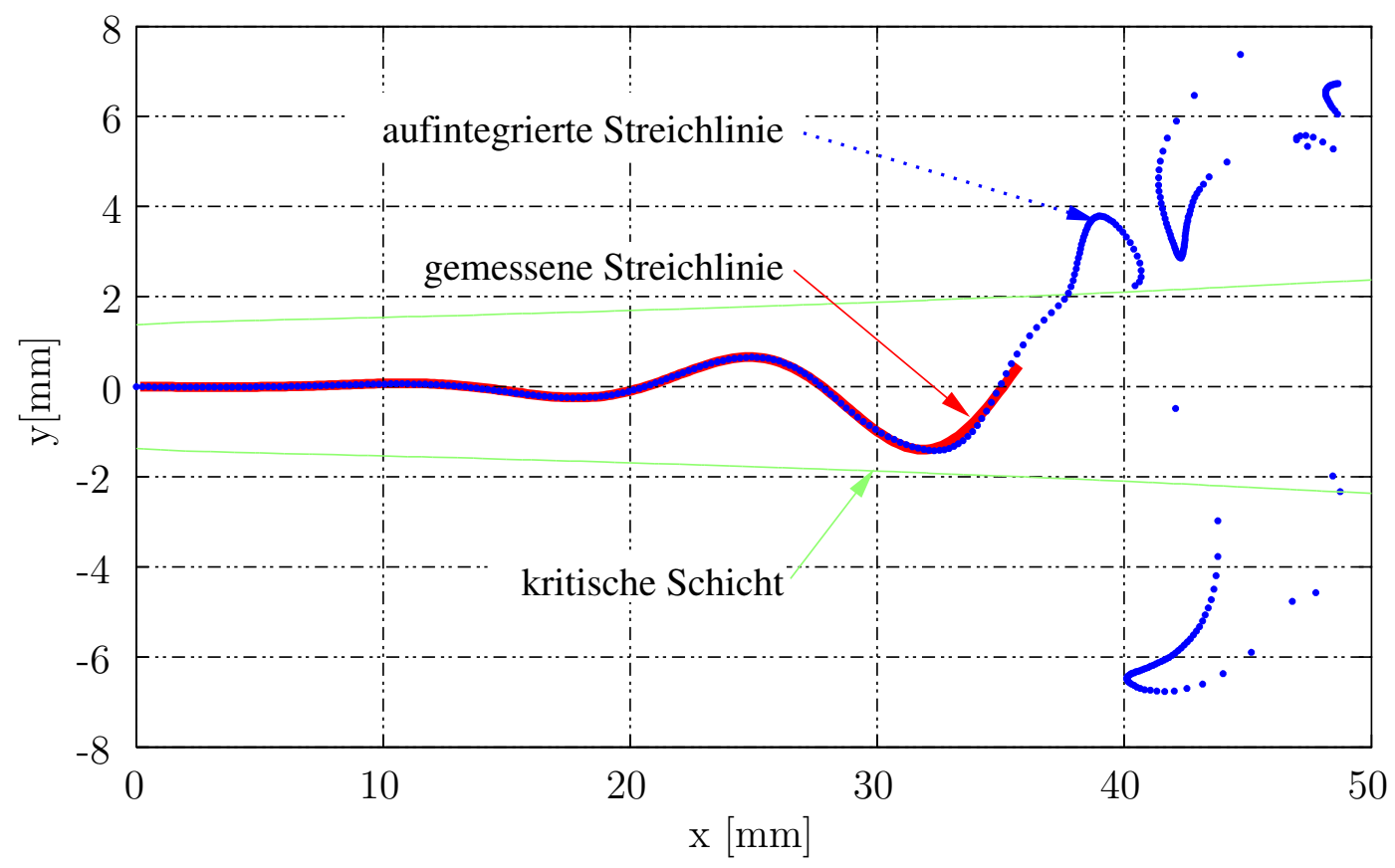

Abb. 3.8: Aufrollen der Streichlinie. Paramter: $f_{\mathrm{s}}=2 \mathrm{~Hz},\left|\hat{a}_{\mathrm{Akt}}\right|=80$ Steps

eine starke Verbreiterung des Profils mit wachsendem Laufweg. Die Anwesenheit des Keils führt zu einer starken Änderung der Strömungsstruktur in seiner Nähe, so dass spätestens dort eine Beschreibung dieser Art nicht mehr gültig ist.

In Anhang B.1.2 sind zwei Ansätze zur Berechnung der Verbreiterung des Strömungsprofils aufgezeigt. Es ergibt sich, dass sowohl die Anfachung des Instabilitätswellenwechselgeschwindigkeitsfeldes als auch die Phasengeschwindigkeit mit zunehmender Anregungsamplitude sinkt.

\subsubsection{Nichtlinearität der Streichlinienintegration}

Der Zusammenhang zwischen dem Strömungsfeld $\underline{U}(x, y, t)$ und der Streichliniemauslenkung $\eta(x, t)$ ist nichtlinear, d.h. man kann unter anderem nicht die zu einer Anregungsamplitude integrierte Streichlinie linear auf eine andere Anregungsamplitude umrechnen, da der Gleichund der Wechselanteil des Strömungsfeldes inhomogen sind. Je größer die Störamplitude ist, desto größer wird die Verzerrung der Streichlinie, da die Teilchen immer stärker in Gebiete mit langsamer Strömungsgeschwindigkeit vorstoßen. Dadurch steigt auch der Anteil der höheren Harmonischen in der Zeitentwicklung von $\eta(x, t)$.

Trotz der Verbreiterung des Profils mittels der in Anhang B.1.2 beschriebenen Methode beginnt die Streichlinie - verglichen mit dem Experiment - sich schon bei sehr kleineren Amplituden aufzurollen. Die scheinbare Dynamik eines Wirbelaufrollens in Abb. 3.8 (vergl. Abb. 3.7) entsteht lediglich durch die Streichlinienintegration. Das unterliegende Strömungsfeld ist eine Superposition des Hauptströmungsprofils und des durch linearisierte Gleichungen ermittelten 
Kapitel 3. Modellierung der konvektiven Instabilität

Wechselanteils, so dass ein prinzipiell nichtlinearer Aufrollprozess dadurch grundsätzlich nicht beschrieben werden kann. Die Struktur der durch die Integration gewonnenen Streichlinienbilder ist den tatsächlich beobachteten Bildern sehr ähnlich. Auffällig ist die Richtungsänderung der numerischen Streichlinie in Abb. 3.8. Die Fluidelemente, die sich gerade mit der Ausbreitungsgeschwindigkeit der Instabilitätswelle bewegen, werden immer gleichphasig zum Wechselfeld transportiert, so dass die Geschwindigkeitsänderung dort besonders groß ist (kritische Schicht). Die Streichlinie, die durch Integration über ein Geschwindigkeitsfeld resultiert, das aus einer Überlagerung von Wechselgeschwindigkeitsfeldern mit unterschiedlichen Anregungsfrequenzen besteht, unterscheidet sich für größere Amplituden erheblich von der Überlagerung der zu den einzelnen Wechselfeldern gehörenden Streichlinien. Die Regelung in Kap. 5 erfordert das Messen von Übertragungsfunktionen durch breitbandige Signale und damit die Messung einer Streichlinie, die aus einer solchen Überlagerung entsteht. Die durch Streichlinienintegration entstehende Nichtlinearität ist in Anhang B.1.3 untersucht. Für den Messprozess ebenfalls von Interesse ist die Abhängigkeit der Streichlinienbewegung vom lateralen Farbstoffinjektionspunkt (siehe Anhang B.1.5).

\subsection{Amplitudenabhängigkeit des Phasengradienten}

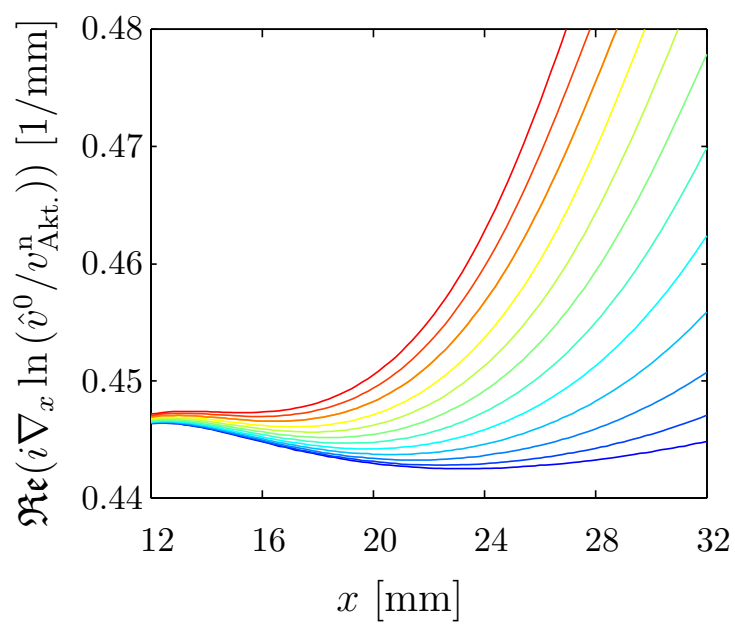

Abb. 3.9: Anstieg des Realteils des komplexen Gradienten mit von blau nach rot äquidistant zunehmender Amplitude von 100 auf 1200 Steps.
Die Strömungsinformation breitet sich mit der Instabilitätswellengeschwindigkeit des Wechselfeldes aus und die gemessene Streichlinie integriert lediglich darüber. Daher hat $\omega / \mathfrak{R e}\left(i \nabla_{x} \ln \left(\tilde{\eta} / \zeta_{\text {Akt. }}^{\mathrm{n}}\right)\right)$ der Streichlinien nicht die allgemeine Bedeutung einer Phasengeschwindigkeit, die eine selbstständige Wellenausbreitung implizieren würde, sondern gibt im Wesentlichen eine durch den Messprozess modifiziert wiedergegebene Phasengeschwindigkeit des Wechselfeldes an.

Der an einem festen Ort gemessene Betrag des Phasengradienten $\mathfrak{R e}\left(i \nabla_{x} \ln \left(\tilde{\eta} / \zeta_{\text {Akt. }}^{\mathrm{n}}\right)\right)$ der Streichlinienauslenkung nimmt mit wachsender Amplitude ab, so dass $\omega / \mathfrak{R e}\left(i \nabla_{x} \ln \left(\tilde{\eta} / \zeta_{\text {Akt. }}^{\mathrm{n}}\right)\right)$ (dargestellt in Abbildungen 3.10 und 3.11 bezogen auf $U_{\mathrm{D}}$ ) zunimmt. Die numerisch berechnete Streichlinienauslenkung $\tilde{\eta}_{\mathrm{WKB}}$ zeigt qualitativ die gleiche Amplitudenabhängigkeit, wobei allerdings auch Unterschiede zwischen gemessener und berechneter Streichlinienauslenkung auftreten (vergl. Abb. 3.11 links und rechts). Aus der Dispersionsrelation des verbreiterten Strömungsprofils ergibt sich dagegen ein Abfall der Phasengeschwindigkeit $\omega / \mathfrak{R e}\left(i \nabla_{x} \ln \left(\hat{v}^{0} / v_{\text {Akt. }}^{\mathrm{n}}\right)\right)$ (Abb. 3.9), da mit Verbreite- 


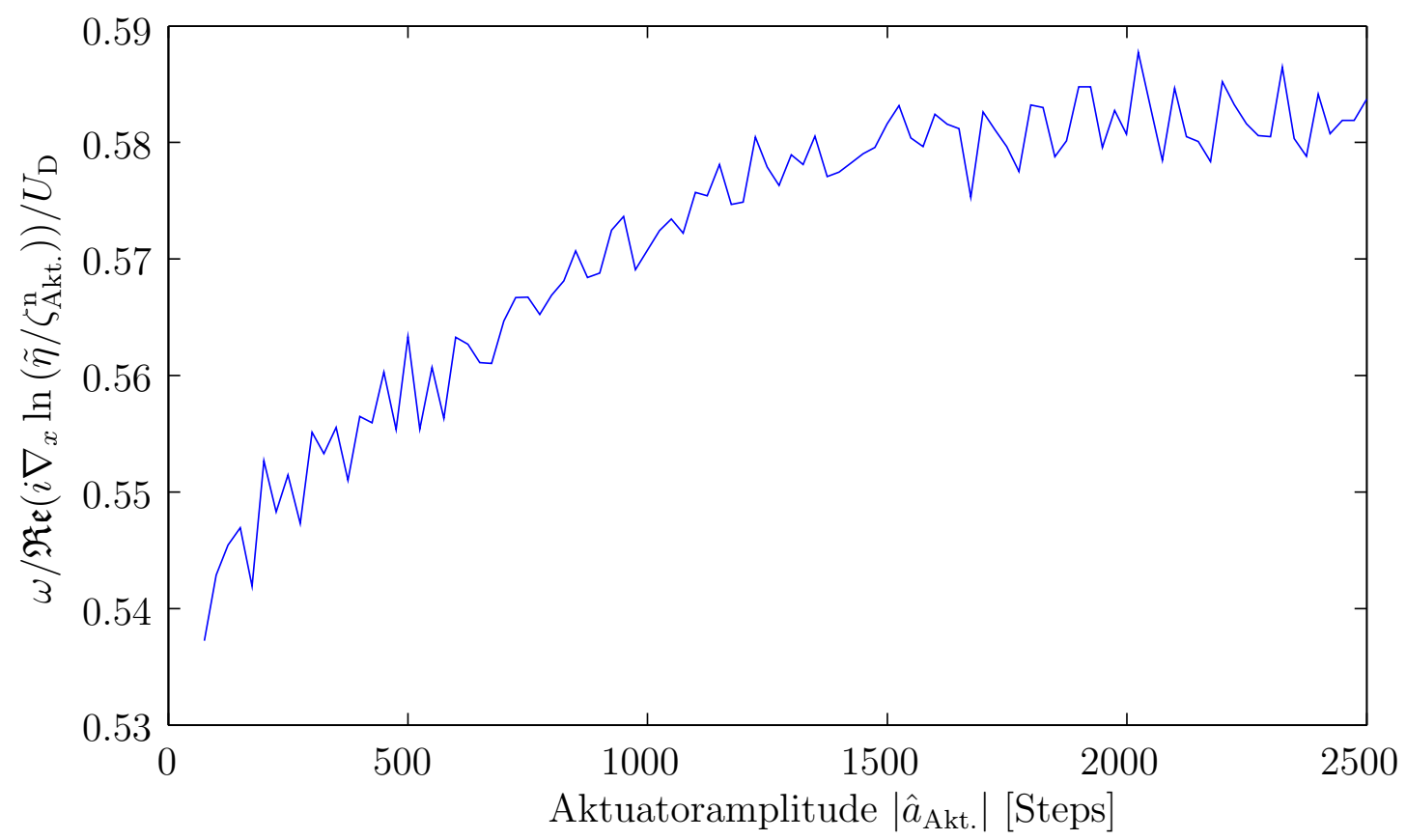

Abb. 3.10: relative „Phasengeschwindigkeit“ $\omega / \mathfrak{R e}\left(i \nabla_{\boldsymbol{x}} \ln \left(\tilde{\boldsymbol{\eta}} / \boldsymbol{\zeta}_{\text {Akt. }}^{\mathrm{n}}\right)\right) / \boldsymbol{U}_{\mathrm{D}}$. Parameter: $x=12 \mathrm{~mm}, f=2 \mathrm{~Hz}$. Die Schwankungen lassen sich auf die Variation der Temperatur zurückführen (siehe Abschnitt 3.3 Gl. (3.12))
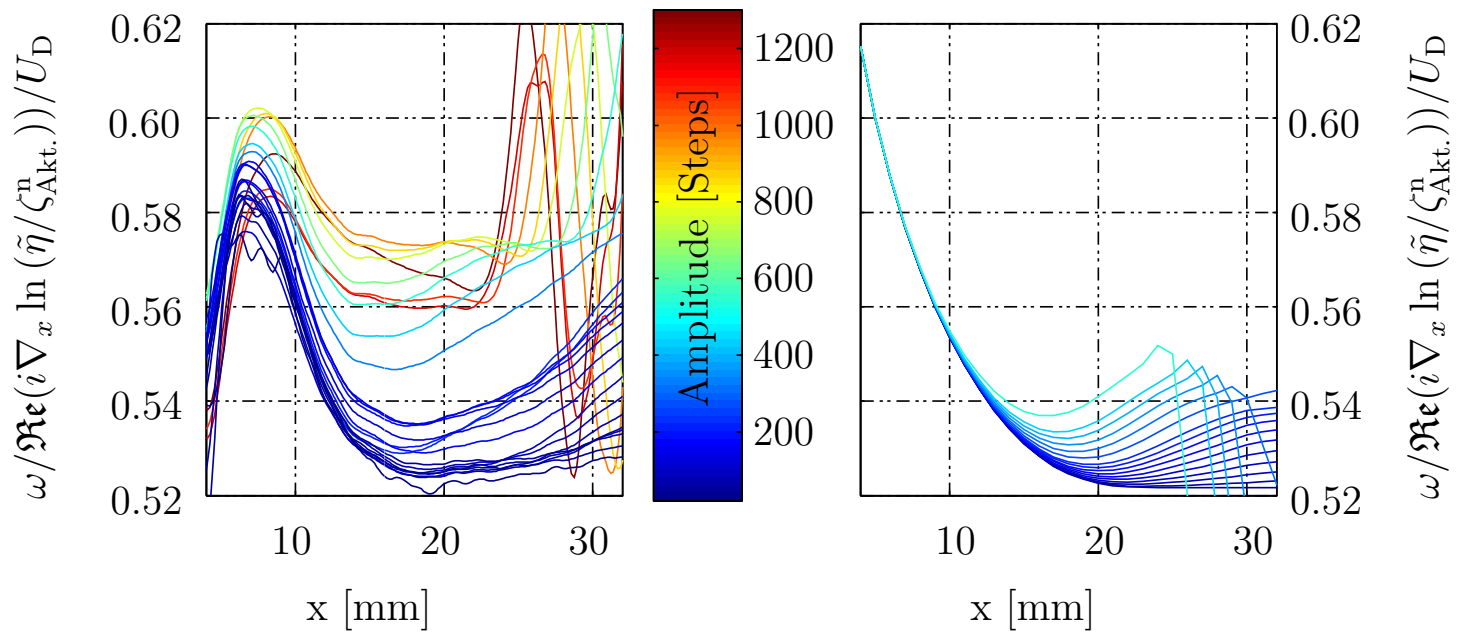

Abb. 3.11: relative „Phasengeschwindigkeit“ $\omega / \mathfrak{R e}\left(i \nabla_{\boldsymbol{x}} \ln \left(\tilde{\boldsymbol{\eta}} / \boldsymbol{\zeta}_{\text {Akt. }}^{\mathrm{n}}\right)\right) / \boldsymbol{U}_{\mathrm{D}}$. Parameter $\left|\hat{a}_{\text {Akt. }}\right|$, links: experimentell, rechts: numerisch 
Kapitel 3. Modellierung der konvektiven Instabilität

rung des Profils die Maximalgeschwindigkeit in der Strahlmitte sinkt. Dieser scheinbare Widerspruch löst sich auf, wenn man bedenkt, dass die Maximalgeschwindigkeit des Strömungsprofils zwar sinkt, jedoch das Profil dabei breiter wird, so dass die Teilchenbahnen auf einem lateral homogeneren Gleichströmungsfeld laufen und so insgesamt schneller werden. Daher nimmt $\omega / \mathfrak{R e}\left(i \nabla_{x} \ln \left(\tilde{\eta} / \zeta_{\text {Akt. }}^{\mathrm{n}}\right)\right)$ mit zunehmender Amplitude zu, obwohl die Phasengeschwindigkeit $\omega / \mathfrak{R e}\left(i \nabla_{x} \ln \left(\hat{v}^{0} / v_{\text {Akt. }}^{\text {n }}\right)\right)$ des Wechselfeldes sinkt.

\subsection{Modellierung der Freistrahlinstabilität durch eine Übertragungskette}

Überblick: Ziel dieses Abschnitts ist die Entwicklung eines signaltheoretischen Modells für die Freistrahlinstabilität, das als Testmodell für die adaptive Regelung dienen kann. Das Modell besteht aus kausalen, in Reihe geschalteten FIR-Filtern mit der Impulsantwort $\Delta s$ (im Frequenzbereich durch $\Delta S$ beschrieben), die jeweils das Übertragungsverhalten der Freistrahlinstabilität vom Ort $x_{\mathrm{k}}$ zum Ort $x_{\mathrm{k}+1}$ beinhalten (siehe Abb. 3.12). Jedes Kaskadenelement beinhaltet zur Nachbildung der Sättigung eine Nichtlinearität.

Die Kausalität der Übertragungsglieder von Ort $x_{1}$ zu Ort $x_{2}, x_{2}>x_{1}$ ist physikalisch gesehen eine starke Einschränkung, da damit eine Ursache-Wirkung-Beziehung in Ausbreitungsrichtung der Strömung gefordert wird, die durch den parabolischen Charakter der partiellen Differenzialgleichung nicht notwendigerweise erfüllt wird. Die Information wird hier durch das Druckfeld an alle Orte gleichzeitig übermittelt. Im Gegensatz zu hyperbolischen partiellen Differenzialgleichungen gibt es hier keine strikte Ausbreitungsrichtung nur in Strömungsrichtung eine Vorzugsrichtung für die Informationsausbreitung. Die Zeitreihen, die man durch Messung beispielsweise der Teilchenauslenkung - an den Orten $x_{1}, x_{2}$ erhält, müssen daher nicht kausal miteinander verknüpft sein; nicht einmal, wenn der eine Ort weit stromab liegt. Bezüglich des Aktuators ist jede Zeitreihe natürlich kausal.

Die Übertragungsfunktionen $\Delta S$ (bzw. $\Delta s$ im Zeitbereich) können aus der numerisch ermittelten Dispersionsrelation oder auch direkt aus experimentellen Daten gewonnen werden. Dabei wird hier diese signaltheoretische Streichlinienauslenkung mit $\eta$ bezeichnet.

Gewinnt man die Impulsantwort $\Delta s$ aus experimentellen Daten, so setzt die Aktuatorübertragungsfunktion $A$ das Eingangssignal $s(t)$ in eine Streichlinienauslenkung $\eta\left(x_{\mathrm{D}}, t\right)$ am Ort $x_{\mathrm{D}} u m$. Das durch die Filterkette propagierende Signal approximiert damit die experimentell gemessene Streichlinienauslenkung.

Gewinnt man die Impulsantwort $\Delta s$ aus der Dispersionsrelation, so braucht man noch einen

experimentell zu bestimmenden Übersetzungsfaktor $A_{\text {Inst. von der Aktuatorauslenkung auf die }}$ Streichlinienauslenkung der Instabilitätswelle bei $x_{\mathrm{D}}$ (Anhang C.3). 
Zur Nachbildung der Sättigung werden nichtlineare Glieder hinzu geschaltet. Benutzt man immer mehr Glieder und verkleinert so den Abstand der Messpunkte kann die Nichtlinearität der Störungsausbreitung immer besser approximiert werden. Allerdings gewinnt der akausale Anteil der Impulsantwort mit abnehmendem Abstand zwischen den Modell-Messpunkten immer größere Relevanz. Der Dynamikbereich der Übertragungskette kann durch Optimierung an den experimentellen Bereich angeglichen werden.

\subsubsection{Bestimmung der Impulsantworten der Übertra- gungskettenglieder}

Die Freistrahlinstabilität soll durch ein Modell beschrieben werden, das aus einer Kaskade von FIR-Filtern besteht, die die Wellenausbreitung beschreiben. In Abständen $\Delta x=x_{i+1}-x_{\mathrm{i}}$ wird jeweils die Impulsantwort für ein FIR-Filter aus der Dispersionrelation des lokalen $\bar{U}(x, y)$-Profils errechnet. Die Übertragungsfunktion kann durch:

$$
\Delta S(x, \omega)_{\Delta x}=\left\{\begin{array}{cl}
e^{i \int_{x}^{x+\Delta x} k(\omega, x) d x} & : \omega \leq \omega_{\text {grenz }} \\
0 & : \quad \omega>\omega_{\text {grenz }}
\end{array}\right.
$$

aus der Freistrahldispersionsrelation $k(x, \omega)$ gewonnen werden. In Abb. 3.13 ist die Übertragungsfunktion, die man aus der Dispersionsrelation mittels Rayleigh-Gleichung erhält, mit der gemessenen verglichen. Die gemessene Dispersionsrelation bricht nicht mit $\omega_{\text {grenz }}$ vollständig ab. Jedoch entsteht bei dieser Grenzfrequenz eine qualitative Änderung des Übertragungsverhaltens. Die Bestimmung der Übertragungsfunktion ist mit großen Unsicherheiten behaftet, da der direkte Übertragungspfad des Aktuators aus den Messdaten abgezogen werden muss und die Streichlinie nicht nur einen Instabilitätswellenanteil beinhaltet (siehe Diskussion in Abschnitt 3.4 und Anhang C.4). Desweiteren nimmt für hohe Frequenzen wegen des Tiefpasscharakters der Instabilität die spektrale Eingangsleistung $P_{\text {in }}(x, f)$ bei der experimentellen Bestimmung von $\Delta S(x, \omega)$ mit dem Laufweg $x$ stark ab. Daher wurde das
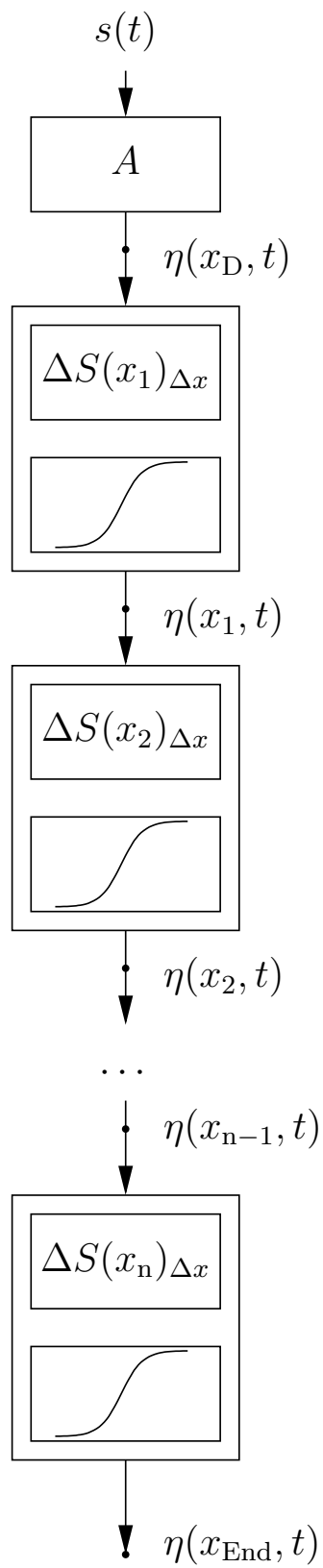

Abbildung 3.12:

Kaskade Aktuatorsignal für hohe Frequenzen stark angehoben (Prewhitening), um genügend Signalstärke zu Orten weit stromab zu transportieren. Durch das Prewhitening und die stromab sich stärker auswirkende Tiefpasscharakteristik sind keine verfälschenden Fensterungen im Frequenzbereich oder Regularisierungsmethoden wie spektrales Abschneiden oder Tikhonov-Regularisierung (siehe Kap. 5) notwendig. Jedoch darf die Aktuatoramplitude für das Prewhitening nicht zu groß gewählt werden, um Nichtlinearität zu vermeiden. Als Kompromiss wird mit einer Amplitude von circa 400 Steps angeregt, die dann im Bereich zwischen 12-15 Hz abfällt. In Abb. 3.13 sind die mit weniger Sicherheit, also mit geringerem spektralen 
Kapitel 3. Modellierung der konvektiven Instabilität

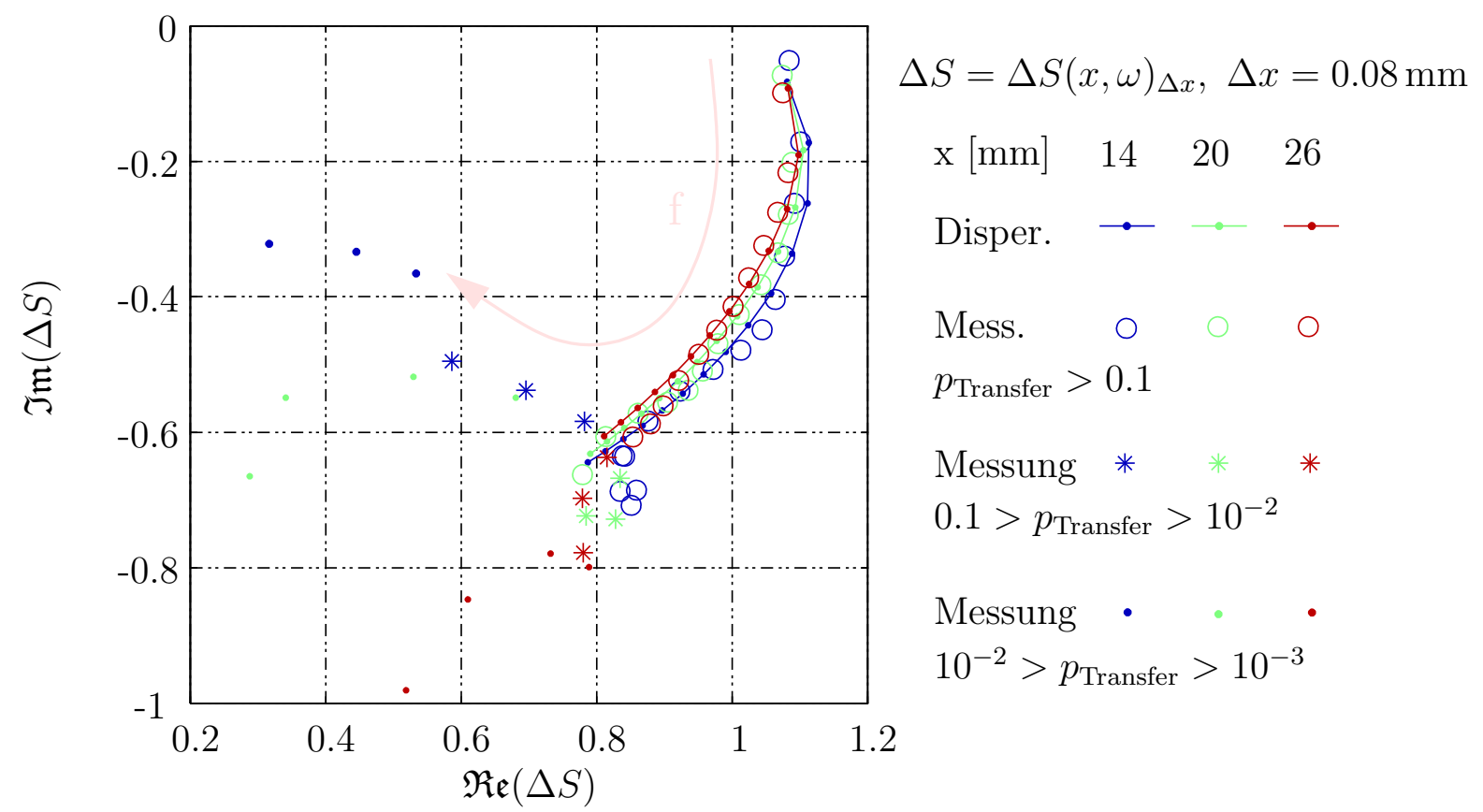

Abb. 3.13: Übertragungsfunktion $\boldsymbol{\Delta} \boldsymbol{H}$ der Strahlauslenkung von den Position $x$ zu der Position $x+\Delta x$ für verschiedene $x$-Positionen. In Pfeilrichtung wächst die Frequenz $f$ an, jeder Punkt entspricht $f=l \cdot \Delta f \quad l \geq 1$ (ohne Gleichanteil $l=0$ ), $\Delta f=\frac{50}{128} \mathrm{~Hz} \approx 0.4 \mathrm{~Hz}$. $U_{\mathrm{D}}=5.4 \mathrm{~cm} / \mathrm{s}$.

Leistungstransferfaktor

$$
p_{\text {Transfer }}(f)=\frac{P_{\text {in }}(f) /\left\langle P_{\text {in }}(f)\right\rangle_{f}}{P_{\text {Akt. }}(f) /\left\langle P_{\text {Akt. }}(f)\right\rangle_{f}}
$$

bestimmten Übertragungsfaktoren speziell gekennzeichnet. Dabei ist $P_{\text {in }}(f) /\left\langle P_{\text {in }}(f)\right\rangle_{\mathrm{f}}$ zusätzlich auf die normierte spektrale Leistungsdichte des Aktuatorsignals $P_{\text {Akt. }}(f) /\left\langle P_{\text {Akt. }}(f)\right\rangle_{\mathrm{f}}$ normiert worden. Der Leistungstransferfaktor fällt oberhalb der Grenzfrequenz der durch den Wellenansatz errechneten Dispersionsrelation stark ab. Dies ist ein Indiz dafür, dass die Wellenausbreitung sich an diesem Modenverzweigungspunkt qualitativ ändert. Die Wellenausbreitung im Sinne eines Exponentialansatzes geht hier in eine andere Form über, deren Ausbreitung stärker gedämpft ist.

Das Filter $\Delta s$ am Ort $x$, das die Instabilitätswelle zum Ort $x+\Delta x$ überträgt, soll - so der Modellansatz - durch

$$
\Delta s(x, t)_{\Delta x}=\left.\mathcal{F}^{-1}\{\Delta S(x, \omega)\}\right|_{t \geq 0}
$$

beschrieben werden, und das Filter $\Delta s$ soll kausal sein, d.h. die Fourier-Rücktransformierte $\mathcal{F}^{-1}\{\Delta S\}$ muss für negative Zeiten verschwinden. 


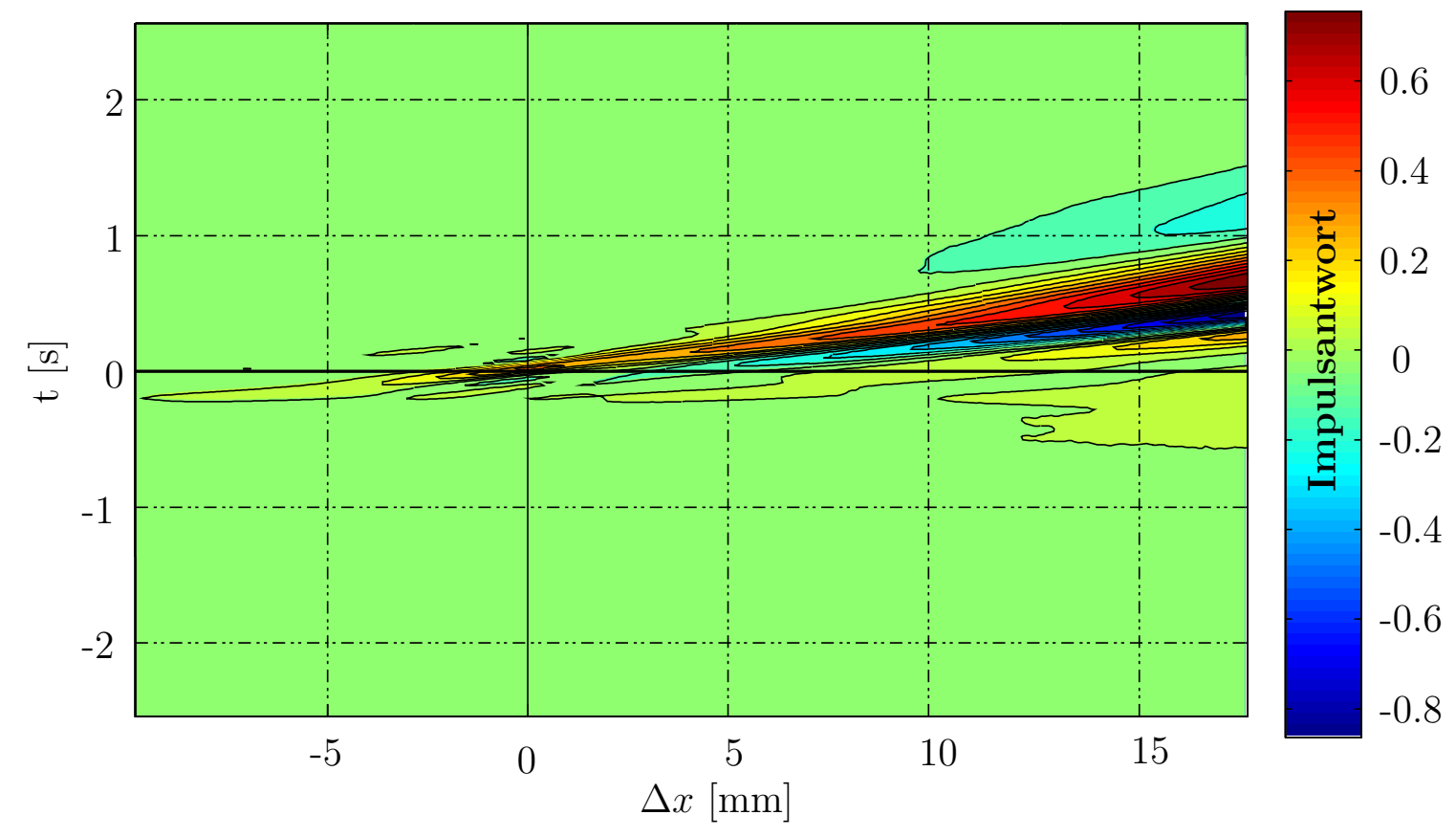

Abb. 3.14: Impulsantworten $\mathcal{F}^{-1}\{\hat{\boldsymbol{\eta}}(\boldsymbol{x}+\Delta \boldsymbol{x}) / \hat{\boldsymbol{\eta}}(\boldsymbol{x})\}$ vom Ort $\boldsymbol{x}=\mathbf{1 0} \mathrm{mm}$ zu den Orten $\boldsymbol{x}+\boldsymbol{\Delta} \boldsymbol{x}$. Die Impulsantwort hat akausale Anteile, $U_{\mathrm{D}}=5.4 \mathrm{~cm} / \mathrm{s}$

Die theoretische Impulsantwort der Filters, das durch die Dispersionsrelation entsteht, ist allerdings akausal, da die Dispersionsrelation bei $\omega_{\text {grenz }}$ abbricht. Auch experimentell fällt die Übertragungsfunktion zu hohen Frequenzen hin zu schnell ab, so dass dabei ebenfalls akausale Anteile in der Impulsantwort entstehen. Die Abbildung 3.14 zeigt die Impulsantwort gemessen vom Ort $x=10 \mathrm{~mm}$ zu den Orten $x+\Delta x$. Selbst für größere $\Delta x$ gibt es Anteile der Impulsantwort für negative Zeiten. Diese akausalen Anteile der experimentell ermittelten Impulsantworten sind etwas kleiner als die Anteile der aus der Dispersionsrelation direkt bestimmten Impulsantwort.

Möchte man den Grenzprozess $\lim \Delta x \rightarrow 0$ durchführen, so lässt sich die Wellenausbreitung mittels kausaler Übertragungsglieder immer schlechter beschreiben. Letztendlich läuft die Frage darauf hinaus, ob man diese Wellenlösung durch immer kleinräumigere konzentrierte Elemente ersetzen kann und so zu einer differenziellen Beschreibung des Systems übergehen kann. Da die Lösungen der Rayleighgleichung für den Freistrahl nur für $\omega<\omega_{\text {grenz }}$ existieren, müssen für den Grenzübergang $\lim \Delta x \rightarrow 0$ auch Lösungen der Eulergleichung, die das Verhalten von $\omega \geq \omega_{\text {grenz }}$ beschreiben, miteingeschlossen werden. Dies soll hier aber nicht weiter verfolgt werden.

Zur Bestimmung der Filterkaskade mit einer Teilung aus $n$ Filtern bis zur Position $x_{\text {End }}=$ $n \cdot \Delta x$ muss die gemessene oder aus der Dispersionsrelation berechnete Übertragungsfunktion 
Kapitel 3. Modellierung der konvektiven Instabilität
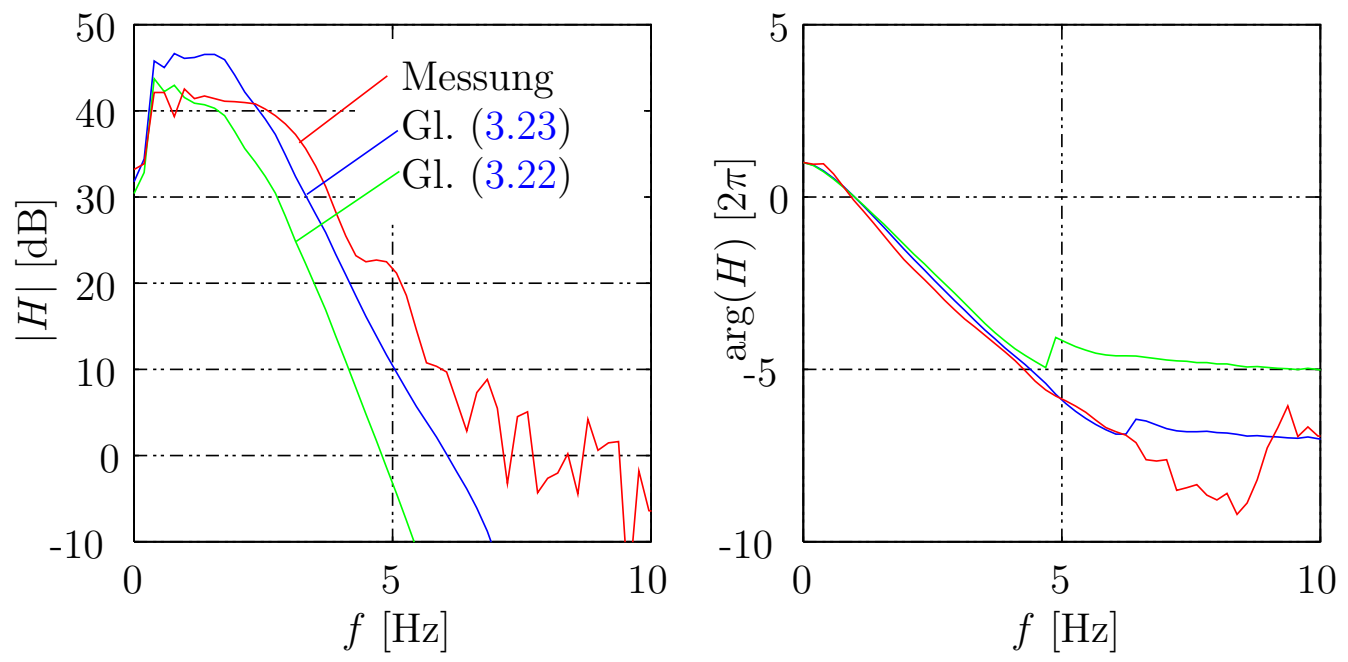

Abb. 3.15: Konvektive Verstärkung der Streichlinienauslenkung von direkt unterhalb der Düse $x_{\mathrm{D}}$ zum Ort $x=26 \mathrm{~mm}$. Im Vergleich ist die Methode Gl. (3.23) - das neue Filter aus den schon berechneten Filtern zu bestimmen - breitbandiger.

$H_{\text {soll }}(x, f)$ durch die Hintereinanderschaltung der $n$ Filter $\Delta s(l \Delta x)_{\Delta x}$ reproduziert werden:

$$
\prod_{l=1}^{n} \mathcal{F}\left\{\Delta s(l \Delta x, t)_{\Delta x}\right\}=H_{\text {soll }}(n \Delta x, \omega) .
$$

Zur Bestimmung der Impulsantworten $\Delta s$ gibt es zwei unterschiedliche Methoden. Entweder versucht man das Filter von Position $l \cdot \Delta x$ zu Position $(l+1) \cdot \Delta x$ direkt durch

$$
\Delta s(l \Delta x, t)_{\Delta x}=\left.\mathcal{F}^{-1}\left\{\frac{H_{\text {soll }}(l \Delta x, \omega)}{\left.H_{\text {soll }}(l-1) \Delta x, \omega\right)}\right\}\right|_{t \geq 0}
$$

zu bestimmen oder man bezieht die schon berechnete vorhandene Filterkette mit ein und berechnet das l-te Filter durch:

$$
\Delta s(l \Delta x, t)_{\Delta x}=\left.\mathcal{F}^{-1}\left\{\frac{H_{\text {soll }}(l \Delta x, \omega)}{\left.\prod_{j=1}^{l-1} \mathcal{F}\left\{\Delta s(j \Delta x, t)_{\Delta x}\right\}\right)}\right\}\right|_{t \geq 0} .
$$

Da der Tiefpasscharakter der Soll-Übertragungsfunktion mit dem Laufweg ausgeprägter wird, bereitet die Invertierung wenig Schwierigkeiten. Man stellt fest, dass mit der letzteren Methode (Gl. (3.23)) die Gesamtübertragungsfunktion etwas besser reproduziert wird (siehe Abb. 3.15). Durch die Beschränkung auf kausale Filter ist nicht mehr jede Betrags- und Phasenkombination möglich (Kramers-Kronig-Relationen), daher geht spektrale Information verloren, die nicht mehr durch nachfolgende Filter zurückgeholt werden kann. In Abb. 3.15 sieht man ferner, dass 

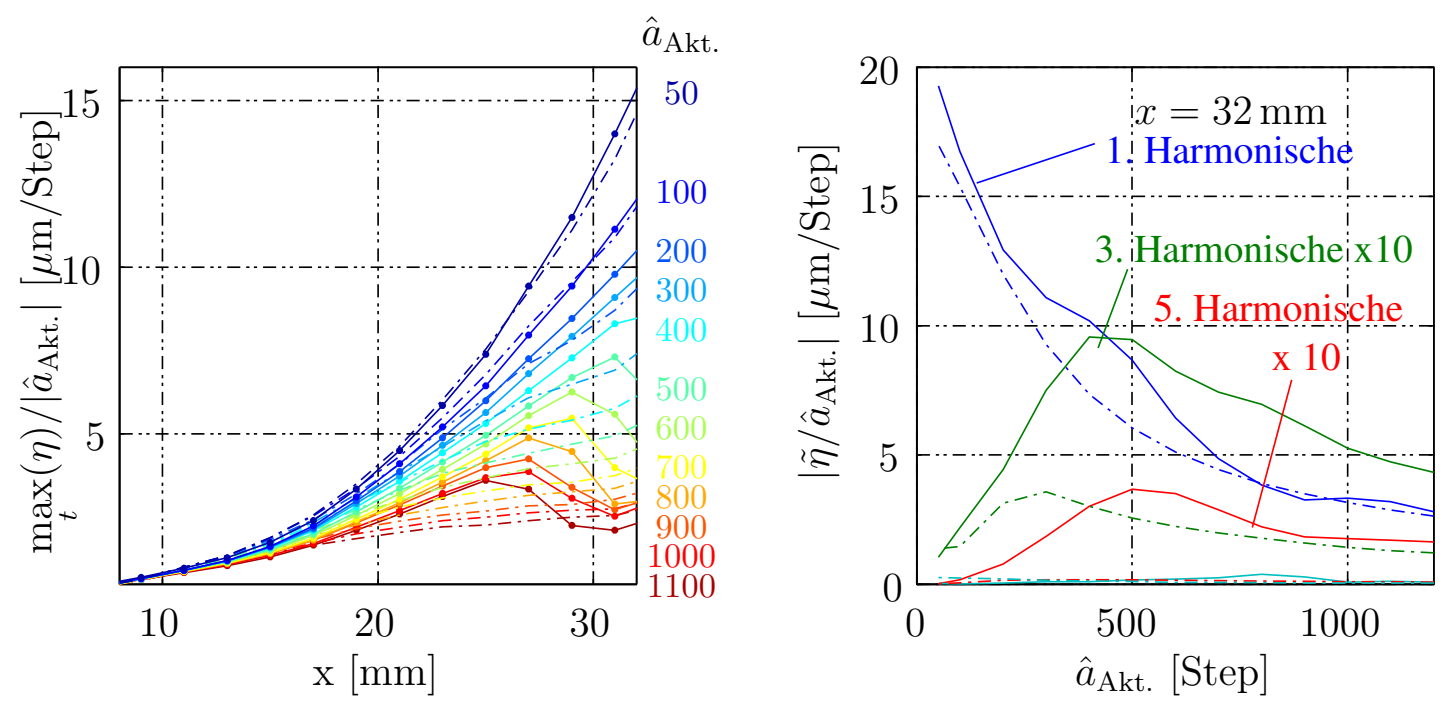

Abb. 3.16: Amplitudenabhängigkeit der Streichlinienauslenkung eines Sinussignals mit $2 \mathrm{~Hz}, U_{\mathrm{D}}=5.36 \mathrm{~cm} / \mathrm{s}$. Messdaten: durchgezogene Linien. Simulation: gestrichelte Linien. Links: die räumliche Entwicklung des Maximums. Rechts: die Amplitude der Harmonischen

die Kausalitätsbedingung zu hohen Verlusten der übertragenen Signalleistung vor allem bei hohen Frequenzen führt, die gemessene Übertragungsfunktion fällt zu hohen Frequenzen weniger steil ab.

\subsubsection{Modellierung der Nichtlinearität}

Das Sättigungsverhalten der Freistrahlinstabilität lässt sich - wie in Abschnitt 3.5 dargestellt nur schwer quantitativ beschreiben. Das qualitative Modell dort legt eine amplitudenabhängige Dämpfung der Anfachung nahe, sowie die Erniedrigung des Phasengradienten, wenn man die Auslenkung der Streichlinie betrachtet.

Als Nichtlinearitäten, die eine Sättigung des Signals $s(x, t)$ bewirken, können zum Beispiel $a \tanh (s(x, t) / a)$ oder Polynome $s(x, t)+\epsilon_{3} s(x, t)^{3}+\epsilon_{5} s(x, t)^{5}+\ldots$ gewählt werden.

Verkleinert man den Abstand $\Delta x$ immer weiter, so kann die Taylorentwicklung bei immer niedrigeren Potenzen abgeschnitten werden, da die Änderung der Auslenkung von Filter zu Filter immer kleiner wird. Damit bleibt $\epsilon_{3}$ als entscheidender Parameter übrig. Dieses Konzept könnte eine einfache nichtlineare Darstellung der sonst sehr schwer zu beschreibenden Dynamik der Sättigung ermöglichen, wenn ein Grenzübergang $\Delta x \rightarrow 0$ möglich wäre. Allerdings führen - wie oben erwähnt - die immer kleineren räumlichen Abstände der Filter zu immer größeren Verlusten in der Gesamtverstärkung der Kaskade.

Es ist möglich die Nichtlinearität auf verschiedene Arten in die Filterkaskade einzubauen, die Vor- und Nachteile einiger Konfigurationen werden in Anhang D.1 beschrieben. Die in Abb. 3.16 


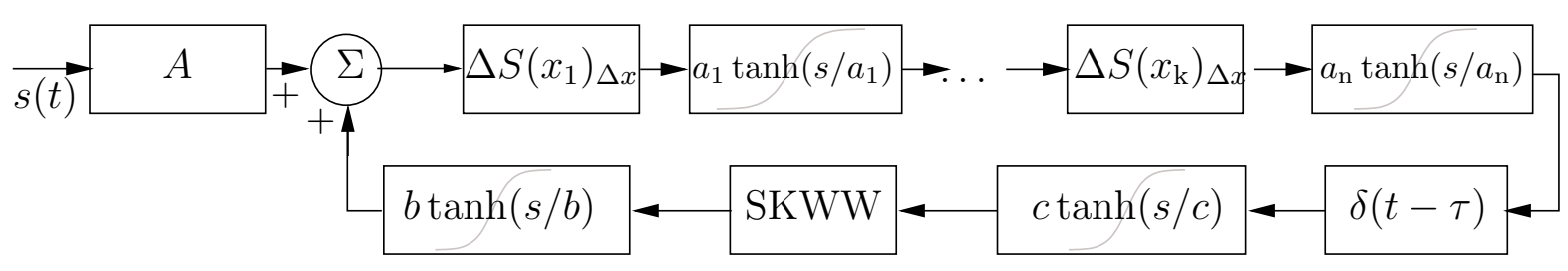

Abb. 3.17: Einfaches Signal-Modell mit Rückkopplung

dargestellte Sättigung der Streichlinienauslenkung mit zunehmender Aktuatoramplitude lässt sich mit der besten im Anhang D.1 beschriebenen Konfiguration annähernd simulieren.

\subsection{Modellierung der globalen Instabilität durch digitale Filter}

Die Moden der globalen Instabilität können mittels der im letzten Abschnitt modellierten konvektiven Instabilität durch zusätzliche Rückkopplung eines Filters von der Keilposition zur Düse dargestellt werden (siehe Abb. 3.17). Dieses Filter repräsentiert die Strahl-KantenWechselwirkung (SKWW) und kann durch die adaptive Regelung ermittelt werden, davor und danach sind noch zwei Nichtlinearitäten geschaltet, mit denen man das Rückkopplungssignal einstellen kann. Von besonderem Interesse ist der Vergleich zwischen Simulation und Experiment bezüglich der Spektren und der Modenübergänge bei Variation des Düse-Keil-Abstands, der im Folgenden mit $L$ bezeichnet ist. Durch Optimierung wird ein optimaler Parametersatz $\left\{a_{1}, \ldots, a_{\mathrm{n}}, b, c\right\}$ in Abb. 3.17 eingestellt, der dann für alle Düse-Keil-Abstände benutzt wird.

Das Modell produziert ein nicht für alle Keilabstände und Strömungsgeschwindigkeiten richtiges Verhalten. Dennoch ist es möglich in gewissen Bereichen gute Übereinstimmung in Bezug auf den Ort $x$ des Modenwechsels und den zu den Moden gehörenden Leistungsspektren zu erzielen, wenn man die Laufzeit der Filterkette etwas verändert. Daher wurde vor das Keilfilter noch ein Laufzeitglied geschaltet. Mit der richtigen Wahl der Laufzeit trifft man dann in Abb. 3.18 (rechts unten) den unteren Frequenz-Peak der Messung, allerdings liegt dann der obere etwas zu niedrig. In der gleichen Abbildung rechts oben ist die dadurch vorausgesagte Frequenz dann auch etwas zu niedrig. Bei kleinerer eingefügter Laufzeit springt das System schon bei $L / d=7$ in die nächst höhere Mode (man erhält dann ein ähnliches Bild wie bei $L / d=8$ ).

Das simulierte Spektrum rechts unten für $L / d=8$ ist durch kurze gezielte Anregung mit der unteren Frequenz entstanden. Die Mode mit der höheren Frequenz, die die größere Anwachsrate hat, verdrängt dann die tiefere Mode. In der Simulation ist das simultane Vorhandensein von zwei Peaks im Leistungsspektrum, die etwa im Frequenzverhältnis 2:1 stehen, schwer einzustellen. Experimentell kann dies beobachtet werden (siehe Kapitel 4). Inwiefern es sich dabei um 

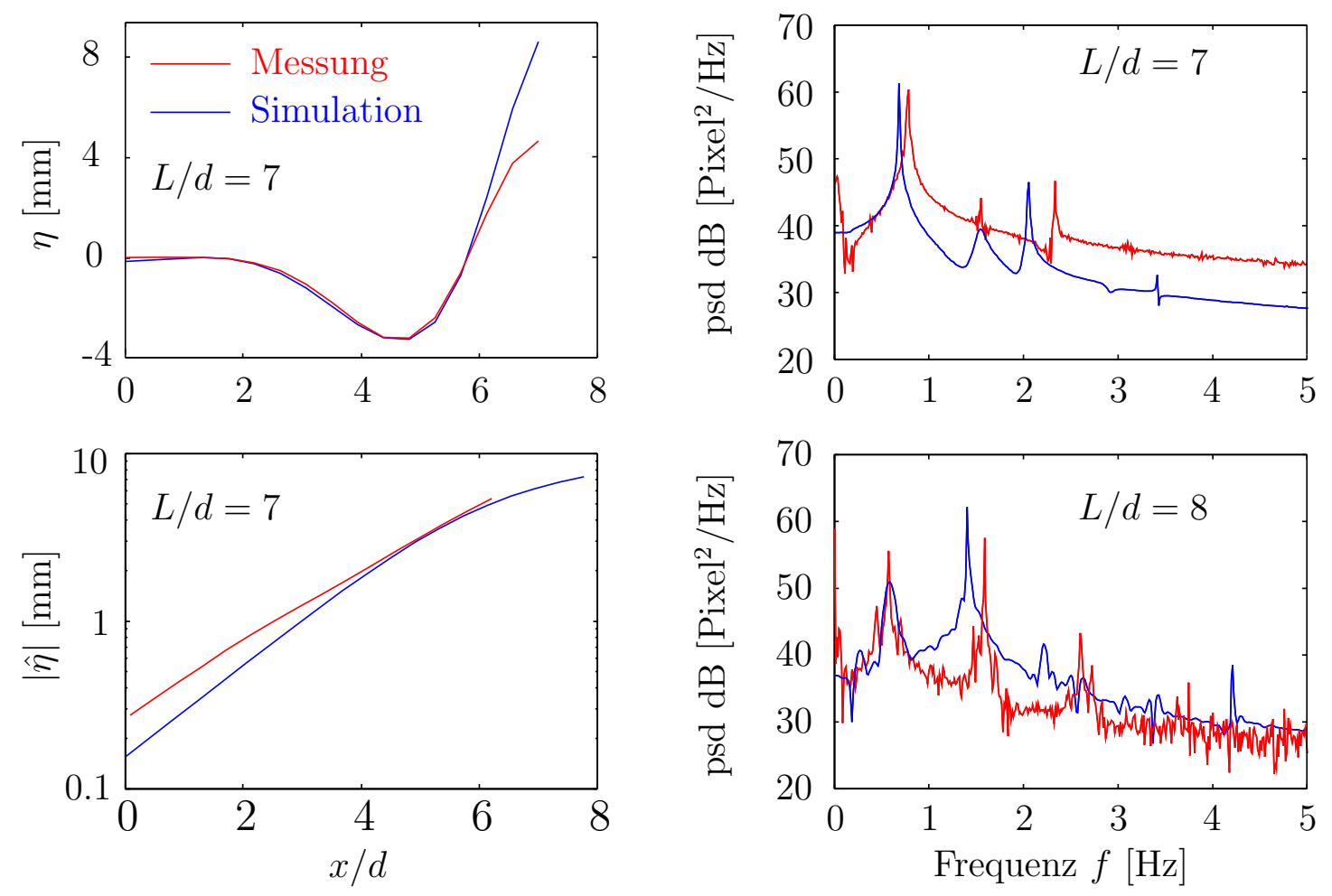

Abb. 3.18: Vergleich zwischen Simulation und Messung für die globale Instabilität bei $L / d=7$ bzw. $L / d=8$. Links: Auslenkung der selbsterregten Schwingung: oben Momentanaufnahme und unten Amplitudenverlauf. Rechts: spektrale Leistungsdichte. $\left(U_{\mathrm{D}}=5.4 \mathrm{~cm} / \mathrm{s}\right.$, $d=4 \mathrm{~mm})$

das Spektrum einer Mode oder das Spektrum zweier unabhängig existierender Moden handelt, wird unter anderem im nächsten Kapitel untersucht. 



\section{Kapitel 4}

\section{Struktur und Dynamik der Moden}

Die ersten Untersuchungen des Strahl-Kanten-Systems wurden von SondHaus (1854 [43]) mittels eines Luftstrahls, der aus einem ebenen Schlitz austritt und auf eine dazu mittig angeordnete Schneide (Kante) trifft, vorgenommen. Die dort oberhalb einer bestimmten Reynoldszahl auftretenden „Schneidentöne“ zeigen bei kontinuierlicher Veränderung des Abstandes zwischen Schlitz und Kante Sprünge in der Frequenz, die durch den Wechsel des Systems von einer Mode in die nächste hervorgerufen werden. Mit zunehmendem Abstand sinkt die Frequenz etwa umgekehrt proportional zum Abstand, um dann bei einem kritischen Abstand in eine höhere Mode mit circa doppelter Frequenz zu springen. Verringert man nun den Abstand wieder, so findet der Modensprung nicht beim selben kritischen Abstand, sondern erst bei einem kleineren statt.

Diese Art der Hysterese lässt sich auch hier beobachten. Die Frequenzverhältnisse sind dabei nicht ganzzahlig. Für höhere Systemzustände wird ein gleichzeitiges Vorhandensein verschiedener „Moden“ beobachtet, d.h. das Leistungsspektrum der Strahlschwingung enthält Frequenzspitzen, die eigentlich einer benachbarten Mode zuzuordnen sind. Dabei stellt sich die Frage, ob es sich um ein Nebeneinander verschiedener einzeln existenzfähiger Moden handelt oder um ein Miteinander der Frequenzkomponenten, so dass von einer einzigen komplexen Mode gesprochen werden kann.

Im Falle nebeneinander existierender Moden muss man davon ausgehen, dass jede Mode einzeln die Randbedingungen an Keil und Düse erfüllt. Die zu den verschiedenen Moden gehörenden Frequenzkomponenten breiten sich dann völlig unbeeinflusst voneinander auf dem Strahl aus. Auch alle anderen Prozesse, wie die Strahl-Kanten-Wechselwirkung und die Anregung der Instabilitätswelle an der Düse, wären trotz der beteiligten Nichtlinearitäten in guter Näherung superponierbar.

Im anderen Fall sind die Frequenzkomponenten miteinander verkoppelt, so dass sie nur gemeinsam die Randbedingungen erfüllen.

Im Abschnitt 4.7 wird die Dynamik der Moden innerhalb der höheren Systemzustände auf Synchronisation untersucht. 


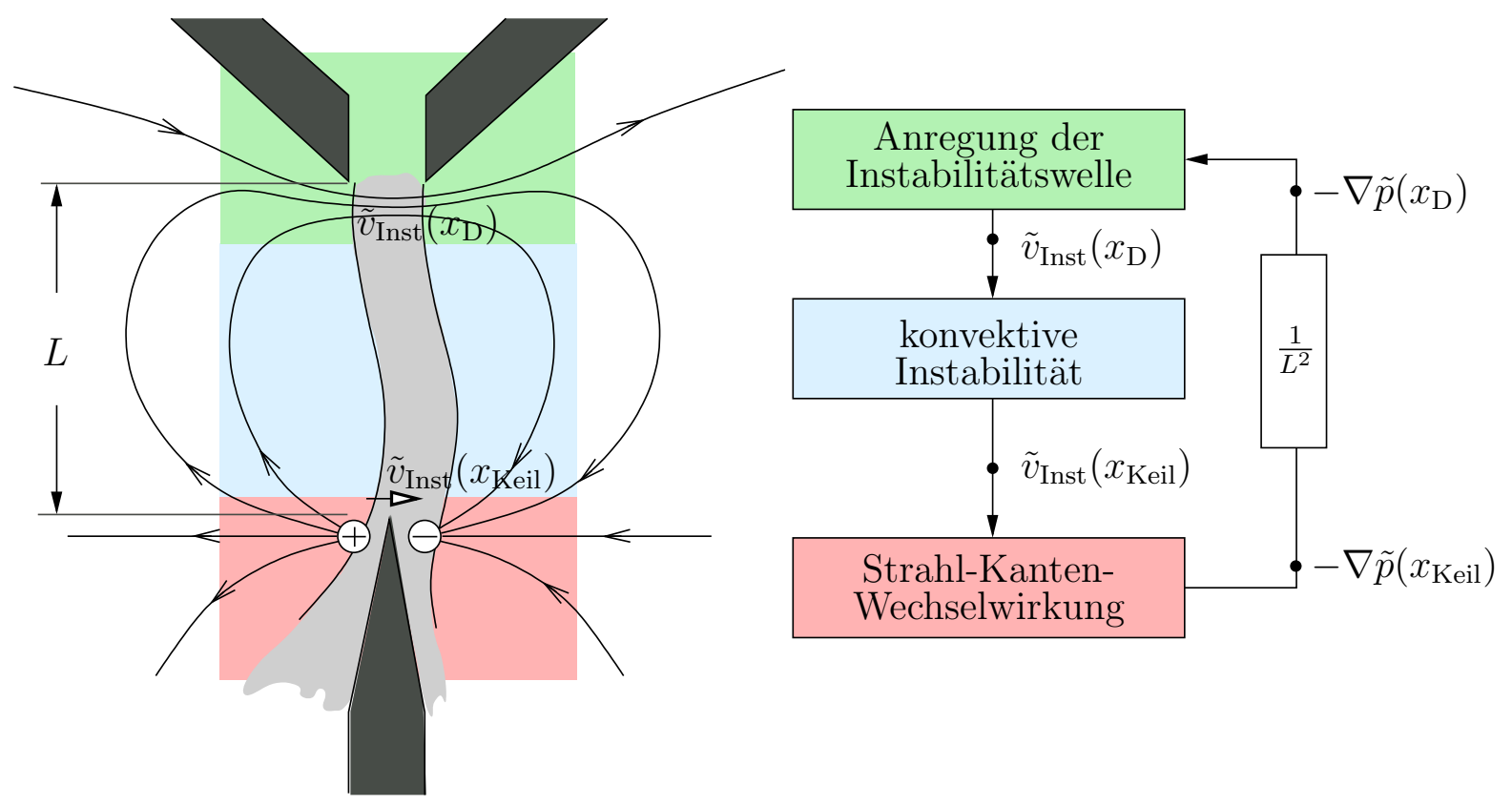

Abb. 4.1: Blockdiagramm des Strahl-Kanten-Systems

Zu Beginn dieses Kapitels wird die Modenstruktur der Strahl-Kanten-Strömung detailliert dargestellt. Die in der Literatur betrachtete Endkorrektur wird diskutiert. Besonderes Augenmerk wird auf das räumliche Anwachsen der Auslenkungsamplitude der Moden zwischen Düse und Keil gelegt.

\subsection{Zerlegung der Strahl-Kanten-Strömung in konzen- trierte Elemente}

Oberhalb einer gewissen Strömungsgeschwindigkeit und eines bestimmten Düse-Keil-Abstandes beginnt das Strahl-Kanten-System selbsterregt zu schwingen. Die Energie dieser Schwingung wird der Gleichströmung durch die Freistrahlinstabilität entzogen. Nimmt man an, dass sich der Rückkopplungsmechanismus der Strahl-Kanten-Strömung durch eine Rückkopplungsschleife beschreiben lässt (POWELL [37, 38] und zusammenfassend [23]), so kann man die Strahl-KantenStrömung in einzelne Übertragungsglieder zerlegen. Die Instabilität des Strahl-Kanten-Systems kann dann wie folgt beschrieben werden:

Eine kleine laterale Strahlauslenkung direkt unterhalb der Düse wird durch die Freistrahlinstabilität verstärkt. Beim Auftreffen des Strahls auf den Keil entsteht durch die Strahl-KantenWechselwirkung ein Druckfeld, das wiederum an der Düse eine neue Störung erzeugt, die dann wieder verstärkt wird. Ist die Gesamtschleifenverstärkung größer als eins bei einer Frequenz, bei 
der das Rückkopplungssignal um eine ganze Zahl von Schwingungsperioden gegenüber dem ursprünglichen Störsignal verschoben ist, so wird die Störung anwachsen bis Nichtlinearitäten sie begrenzen und so die Gesamtschleifenverstärkung genau eins ist. Die Störung erfasst nach einer gewissen Zeit die gesamte Strömung. Die stationäre Strömung ist damit global instabil. Die Beschreibung dieser globalen Instabilität legt eine Aufteilung des Systems wie in Abb. 4.1(rechts) nahe. Die Rückkopplung geschieht akustisch durch ein Druckdipolfeld. Es liegt also keine in die Strömungsdynamik unauflöslich integrierte Rückkopplung vor, wie sie zum Beispiel bei der absoluten Instabilität der Kármánschen-Wirbelstraße nahe des senkrecht angeströmten Zylinders [7] vorkommt.

Im Folgenden werden die Eigenschaften der einzelnen Systembausteine zusammengefasst.

\section{Anregung der Instabilitätswelle}

Die von der Strahl-Kanten-Wechselwirkung und dem Aktuator ausgehenden Kraftfelder $\left(-\nabla \tilde{p}_{\text {SKww }}\right.$ bzw. $\left.-\nabla \tilde{p}_{\text {Akt. }}\right)$ wirken in Düsennähe auf den austretenden Strahl ein und erzeugen ein Wechselgeschwindigkeitsfeld unterhalb der Düse, das die Instabilitätswelle anregt. In dem Blockschaltbild Abb. 4.1 ist der Anregungsmechanismus durch die Übertragungsfunktion von $-\nabla \tilde{p}\left(x_{\mathrm{D}}\right)$ auf die Wechselgeschwindigkeit $\tilde{v}_{\text {Inst }}\left(x_{\mathrm{D}}\right)$ der Instabilitätswelle unterhalb der Düse charakterisiert. Dabei sei $-\nabla \tilde{p}\left(x_{\mathrm{D}}\right)$ ein charakteristischer Druckgradient in Düsennähe, den man ohne überlagerte Strahlströmung vorfinden würde; auch $\tilde{v}_{\text {Inst }}\left(x_{\mathrm{D}}\right)$ ist eine charakteristische Wechselgeschwindigkeit, die das Strömungsfeld der Instabilitätswelle in unmittelbarer Düsennähe charakterisiert, also nicht das Nahfeld der Düse enthält.

Trotz der räumlich leicht unterschiedlichen Verteilungen der Potenzialfelder der Strahl-KantenWechselwirkung und des Aktuators, sollte sich die Wirkung der beiden Anregungsmechanismen in Bezug auf die Anregung der Instabilitätswelle nicht deutlich unterscheiden. Daher kann man dem Druckfeld der Strahl-Kanten-Wechselwirkung eine effektive Wechselschnelle $\tilde{v}_{\text {Akt. }}$ des Aktuators zuordnen. Für die Umsetzung der Aktuatorschnelle in die Instabilitätswelle fehlt allerdings ein Modell. Der Zusammenhang zwischen diesen Größen ist in Anhang C dargestellt.

\section{Konvektive Instabilität}

Die Instabilitätswelle breitet sich stromab aus und wächst dabei stark an. Dieser Verstärker ist, wie in Kap. 3 dargestellt, stark nichtlinear und sättigt sich selbst. Das mittlere Strahlprofil verbreitert sich durch das Anwachsen der Wechselamplituden, so dass die Anfachung mit zunehmender Amplitude abnimmt. Das räumliche Anwachsen der Instabilitätswelle mit dem Laufweg wird in Abschnitt 4.5 behandelt. Die maximale konvektive Verstärkung des Freistrahls pro Düsenbreite hängt nur wenig von der Strömungsgschwindigkeit und $x$-Position ab und beträgt circa 6-10 dB/d. Der Phasengradient der Wechselgeschwindigkeit $\mathfrak{R e}\left\{i \nabla_{x} \ln \left(\tilde{v} / v_{\text {Akt. }}^{\mathrm{n}}\right)\right\}$ ist amplitudenabhängig und steigt mit wachsender Amplitude leicht an. 


\section{Strahl-Kanten-Wechselwirkung und Rückkopplung}

Die Wechselwirkung zwischen Instabilitätswelle und Keil führt zu einem dipolartigen Druckfeld. Die Stärke und die effektive räumliche Position dieses Dipols in Abhängigkeit von der Strahlauslenkung und der Frequenz sind schwierig zu ermitteln. Ein Modell für die Strahl-Kanten-Wechselwirkung kann für kleine Wechselamplituden durch das Überführen der Freistrahlinstabilität in eine Mode gewonnen werden, die am Keil wieder anlegt(„,mode matching“/WienerHopf-Methode [8]). Für größere Amplituden zerfällt der Strahl in Wirbel (siehe Simulation von OHRING [34]). Eine umfassende theoretische Beschreibung der Strahl-Kanten-Wechselwirkung muss daher die gesamte räumliche Wirbeligkeitsverteilung mitberücksichtigen.

In einer einfachen Beschreibung der Strahl-Kanten-Wechselwirkung bremst der Keil die periodische Querbewegung des Strahls ab. Dafür muss der Keil eine Kraft auf das ihn umgebende Medium ausüben. Dies führt zu einem akustischen Dipolfeld, das in Abb. 4.1 für den nach rechts laufenden Strahl eingezeichnet ist. Die räumliche Position des effektiven Dipols auf dem Keil liegt circa eine viertel Wellenlänge stromab ([24, 25]). Da der senkrecht zum Keil durch die Strahlströmung transportierte Impuls vom Keil „absorbiert“ wird, ist die Dipolstärke für sehr kleine Strahlauslenkungen proportional zur lateralen Geschwindigkeit und proportional der Längsgeschwindigkeit des Strahls.

Die Strahl-Kanten-Wechselwirkung kann als Übertragungsfunktion von der Auslenkung der Streichlinien direkt oberhalb des Keils zur Streichlinienauslenkung direkt unterhalb der Düse durch die adaptive Regelung (siehe Kapitel 5) ermittelt werden. Allerdings sind bei dieser indirekten Methode Sekundärprozesse beteiligt, wie die Modellannahmen zur Regelung (Annahme einer Zielübertragungsfunktion) und Zwischenprozesse, wie z. B. die Umsetzung der Streichlinie in das Wechselfeld. Durch deren Einfluss sind die Ergebnisse schwer zu interpretieren.

In Anhang E.1 wird das in diesem Abschnitt behandelte Modell der Strahl-Kanten-Strömung auf die Streichlinienauslenkung bezogen. Dort werden die in Kapitel 3 dargestellten Phasenänderungen abgeschätzt, die sich aufgrund der Messung der Streichlinie anstelle des Geschwindigkeitsfeldes ergeben.

\subsection{Messverfahren}

Aus der Auslenkung der Mitten-Streichlinie $\tilde{\eta}(x, t)=\left.\tilde{\eta}\left(x, t, x_{\text {start }}, y_{\text {start }}\right)\right|_{\left(x_{\text {start }} \approx-\infty, y_{\text {start }}=0\right)}$ kann durch zeitliche Fouriertransformation das Amplitudenspektrum bestimmt werden. Die spektrale Amplitudendichte um die Spitzenwerte $\omega_{\mathrm{i}}$ des Spektrums wird in einer kleinen Umgebung aufintegriert und in der komplexen Amplitude $\hat{\eta}_{\mathrm{i}}(x)$ zusammengefasst, so dass die Streichlinienbewegung aus wenigen Komponenten bei den diskreten Frequenzen $\omega_{\mathrm{i}}$ zusammengesetzt 
werden kann:

$$
\begin{array}{cl}
\tilde{\eta}(x, t)= & \int_{-\infty}^{\infty} \hat{\eta}(x, \omega) e^{i \omega t} d t=\int_{-\infty}^{\infty}|\hat{\eta}(x, \omega)| e^{i\left(\omega t-\varphi_{\eta}(x, \omega)\right)} d t \approx \sum_{i}\left|\hat{\eta}_{\mathrm{i}}(x)\right| e^{i\left(\omega_{\mathrm{i}} t-\varphi_{\eta_{\mathrm{i}}}(x)\right)} \\
\operatorname{mit} & \varphi_{\eta}(x, \omega)=-\arg \left\{\hat{\eta}(x) / \hat{\eta}\left(x_{\mathrm{D}}\right)\right\}=\int_{x_{\mathrm{D}}}^{x} \mathfrak{R e}\left\{i \nabla_{x} \ln \left(\hat{\eta}\left(x^{\prime}\right) / \hat{\eta}\left(x_{\mathrm{D}}\right)\right)\right\} d x^{\prime} .
\end{array}
$$

Dabei gibt es keine ausgezeichnete Normierung für die Streichlinienauslenkung, so dass $\hat{\eta}\left(x_{\mathrm{D}}\right)$ herangezogen wird. Im Fall der hier benutzten diskreten Fouriertransformation werden die am meisten Leistung tragenden Frequenzen in jedem relevanten Frequenzband $i$ ausgewählt, die zugehörigen Peakfrequenzen $\omega_{\mathrm{i}}$ durch quadratische Interpolation des log-Spektrums bestimmt und schließlich die zugehörigen komplexen Amplituden $\hat{\eta}_{\mathrm{i}}(x)$ berechnet.

Aus den Peakfrequenzen $\omega_{\mathrm{i}}$ wird die für die Mode $m$ charakteristische Frequenz $\omega_{\mathrm{m}}$ ausgewählt, indem zusätzlich die räumliche Verteilung der Strahlauslenkung einbezogen wird. Dies ist notwendig, da bei größeren Keilabständen, insbesondere ab Mode II, mehrere Peaks ähnlicher Leistung im Spektrum zu finden sind, deren Frequenzen nicht harmonisch liegen, also nicht zur gleichen Mode gehören können. Anhand der räumlichen Schwingungsform kann entschieden werden, welches $\omega_{\mathrm{i}}$ dem Systemzustand zugeordnet wird. Dieses Problem wird in Abschnitt 4.6 ausführlich behandelt und die damit verbundene Koexistenz von Moden wird in Abschnitt 4.7 untersucht.

Für die Ergebnisse dieses Kapitels wurden zwei unterschiedlich breite Düsen benutzt: $d=2 \mathrm{~mm}$ und $d=4 \mathrm{~mm}$ mit einer Tiefe von jeweils $36 \mathrm{~mm}$. Der Keilabstand kann mit einer Genauigkeit von etwa 2/10 mm eingestellt werden. Desweiteren wird die Temperatur und die Durchflussgeschwindigkeit gemessen, die man zur Berechnung der Reynoldszahl und der Strouhalzahl benötigt.

\subsection{Räumliche Struktur: Phasenbeziehungen und Wel- lenzahl}

Die gemessene Phase von $\tilde{\eta}$ ist in Abb. 4.2 als Funktion des Laufweges dargestellt. Der Gradient der Phase ist aufgrund der Nahfelder an Düse und Keil und der sich mit dem Laufweg ändernden Ausbreitungsgeschwindigkeit der Instabilitätswelle nicht konstant. Daher ist die Zuordnung einer Wellenzahl $k_{\mathrm{m}}$ zu der Mode $m$ abhängig davon, welche Aussagen man mit der Wellenzahl verbinden möchte.

Wenn man die Wellenzahl einfach als eine für die Mode charakteristische Zahl betrachtet, dann ist die Phasendifferenz zwischen der Strahlauslenkung direkt unterhalb der Düse und der direkt oberhalb des Keils bezogen auf die Lauflänge $L$ eine solche Wellenzahl (siehe Abb. 4.1). Diese wird in Abschnitt 4.3.1 untersucht.

Schließt man die Nahfelder aus der Betrachtung aus, so kann man den Gradienten der Phase der Streichlinienauslenkung dort bilden, wo die Phase näherungsweise linear verläuft. Damit 


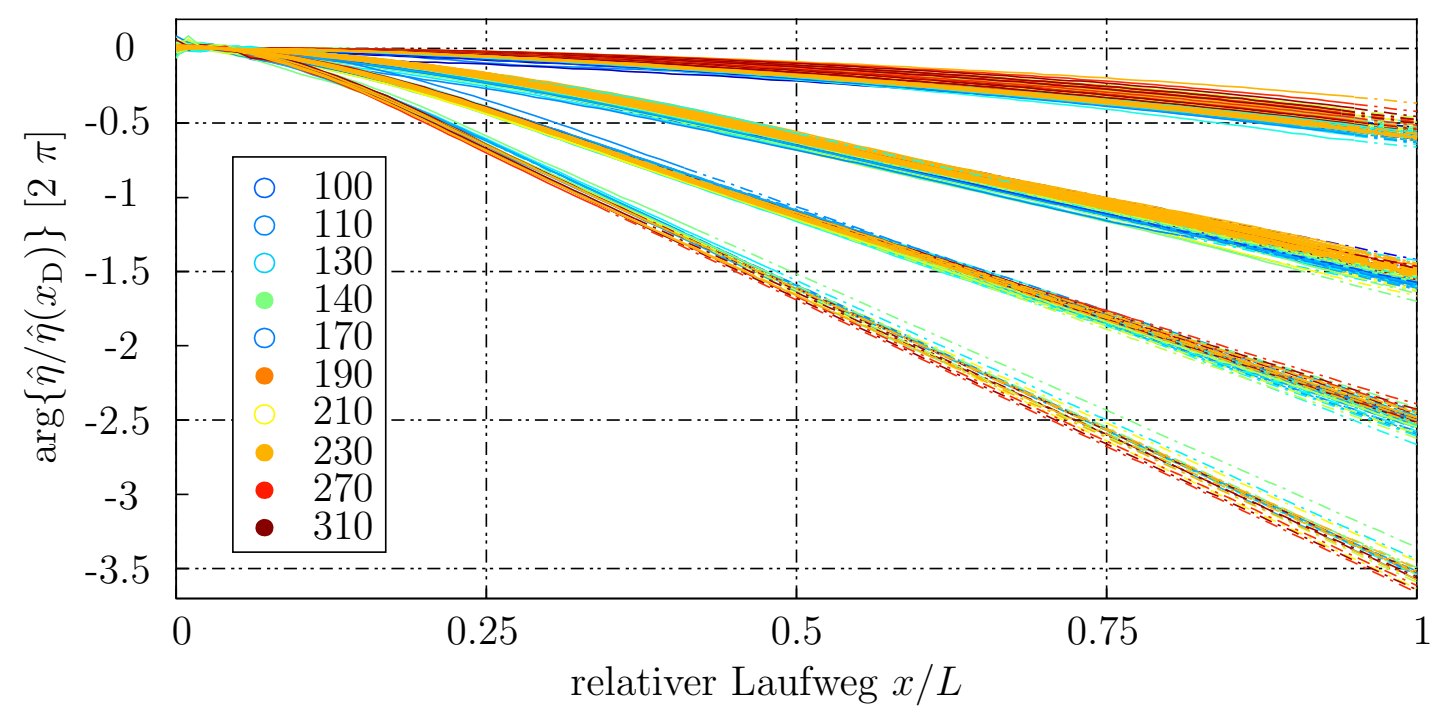

Abb. 4.2: Phase in Abhängigkeit des normierten Laufwegs. Für verschiedene Moden und Reynoldszahlen sind die Phasen der Hauptfrequenzkomponenten linear bis zum Keil extrapoliert. Gestrichelte Linien zeigen den extrapolierten Anteil, die Farbskala kodiert die Reynoldszahl (gefüllte Symbole $d=4 \mathrm{~mm}$, sonst $d=2 \mathrm{~mm}$ ).

kann die Phasenänderung aufgrund der Instabilitätswellenausbreitung von den Nahfeldern entkoppelt werden. Ein besseres, aber aufwendigeres Verfahren besteht darin, die WKB-Lösung (d.h. die Streichlinie, die auf Basis des WKB-Feldes bestimmt worden ist,) im Fernfeld an die Streichlinienauslenkungen anzupassen und in das Nahfeld zurück zu extrapolieren. Damit hätte man eine physikalisch motivierte Definition des Nahfelds.

Der mittlere Phasengradient - gebildet außerhalb der Nahfelder - gibt somit ein Maß für die Gesamtphasendrehung der Instabilitätswelle an. Da die gesamte Rückkopplungsschleife eine Phasendrehung von $-m \cdot 2 \pi$ hat, muss die Phasendrehung, die nicht von der Instabilitätswelle herrührt, durch die Wechselwirkung des Strahls mit dem Keil und der Anregung der Instabilitätswelle an der Düse entstehen. Diese Sichtweise wird in Abschnitt 4.3.2 verfolgt und oft in der Literatur diskutiert.

\subsubsection{Phasenbeziehung zwischen den Streichlinienauslenkungen an Düse und Keil}

Untersucht man die Phasenbeziehungen innerhalb des selbsterregt schwingenden Strahl-KantenSystems, so macht man zunächst eine sehr einfache Feststellung: Die Streichlinienauslenkungen $\hat{\eta}\left(x_{\mathrm{D}}\right)$ direkt unterhalb der Düse und $\hat{\eta}\left(x_{\text {Keil }}\right)$ direkt oberhalb des Keils sind für alle Moden etwa gegenphasig, wie in Abb. 4.2 zu sehen. In Abb. 4.3 ist die Phasendifferenz als Funktion von $L / d$ aufgetragen. 


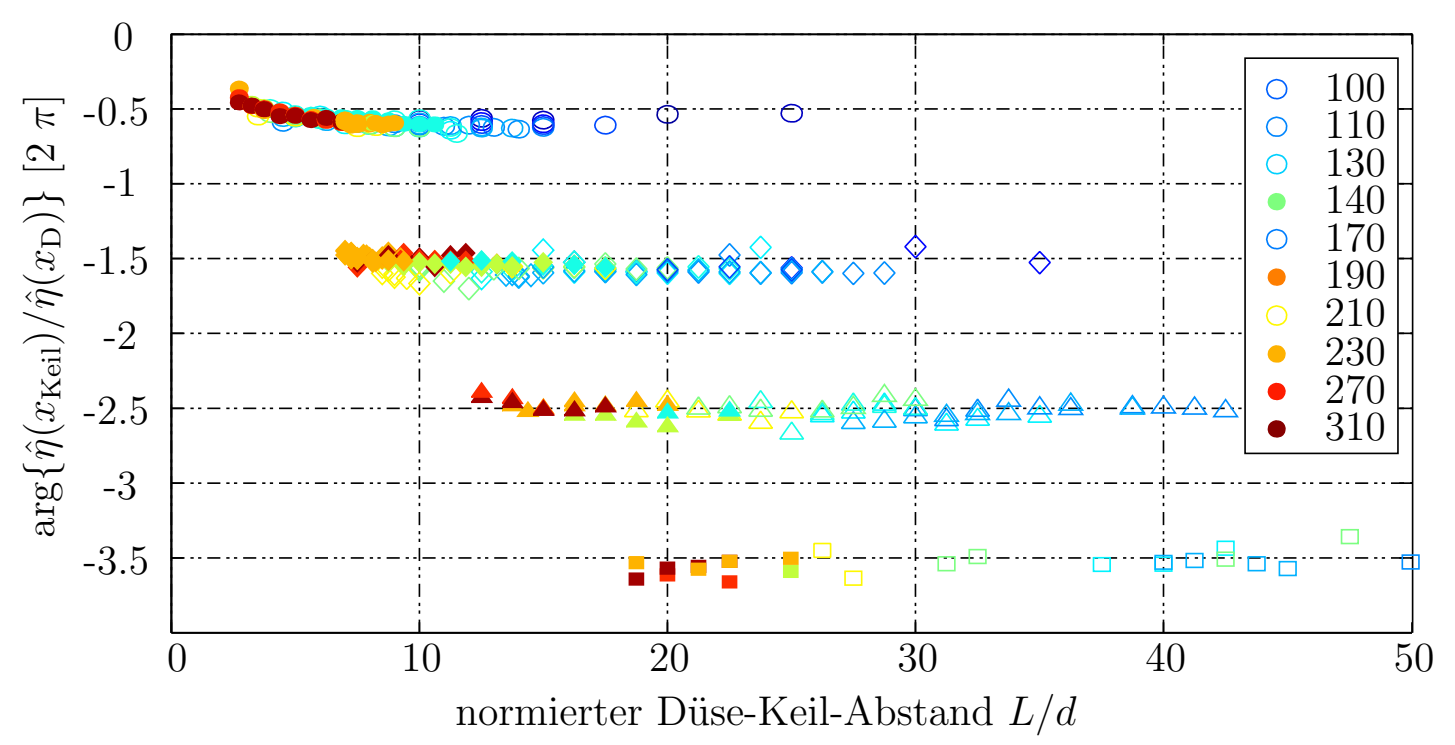

Abb. 4.3: Phasendifferenz gegen den normierten Abstand zwischen Düse und Keil. $\circ$ Mode I, $\diamond$ Mode II, $\triangle$ Mode III, $\square$ Mode IV; gefüllte Symbole $d=4 \mathrm{~mm}$, sonst $d=2 \mathrm{~mm}$; die Farbe kodiert die Reynoldszahl von blau nach rot.

Damit erhält man als Phase der Strahl-Übertragungsfunktion einschließlich aller Nahfelder an Keil und Düse $S=\frac{\hat{\eta}\left(x_{\text {Keil }}\right)}{\hat{\eta}\left(x_{\mathrm{D}}\right)}$ :

$$
\arg \left\{\frac{\hat{\eta}\left(x_{\text {Keil }}\right)}{\hat{\eta}\left(x_{\mathrm{D}}\right)}\right\} \approx-(2 m-1) \pi
$$

In Abb. 4.4 ist diese Phase modulo $2 \pi$ in einer vergrößerten Darstellung als Funktion von $L / d$ gezeigt. Für jeweils feste Modenordnung $m$ erkennt man einen leichten Anstieg der Phasenverzögerung zu größeren $L / d$. Die Übertragungsfunktion $\frac{\hat{\eta}\left(x_{\text {Keil }}\right)}{\hat{\eta}\left(x_{\mathrm{D}}\right)}$ kann in eine effektive Aktuatorübertragungsfunktion $\frac{\hat{\eta}\left(x_{\text {Keil }}\right)}{\hat{a}_{\text {Akt. }}^{\text {ae }}}$ umgerechnet werden, die das Druckdipolfeld der Strahl-Kanten-Wechselwirkung charakterisiert. Dazu wird die Übertragungsfunktion von der äquivalenten Aktuatorauslenkung $\hat{a}_{\text {Akt. }}^{\text {ae }}$ auf die Streichlinienauslenkung durch die Strahlrezeptivität $A$ aus Gleichung (C.14) einbezogen:

$$
\frac{\hat{\eta}\left(x_{\mathrm{Keil}}\right)}{\hat{a}_{\mathrm{Akt} .}^{\mathrm{ae}}}=\frac{\hat{\eta}\left(x_{\mathrm{Keil}}\right) A}{\hat{\eta}\left(x_{\mathrm{D}}\right)}=A S .
$$

Der oben erwähnte leichte Anstieg der Phasenverzögerung für eine bestimmte Mode wird durch die Einbeziehung der Strahlrezeptivität zum Teil kompensiert.

Die einfache Phasenbeziehung von $-\pi$ zwischen Düse und Keil ist erstaunlich, da die einzelnen beteiligten Prozesse im Detail sehr unterschiedliche Abhängigkeiten von der Strouhalzahl und dem Keilabstand haben. Man würde erwarten, dass sich eine einfache Phasenbeziehung 

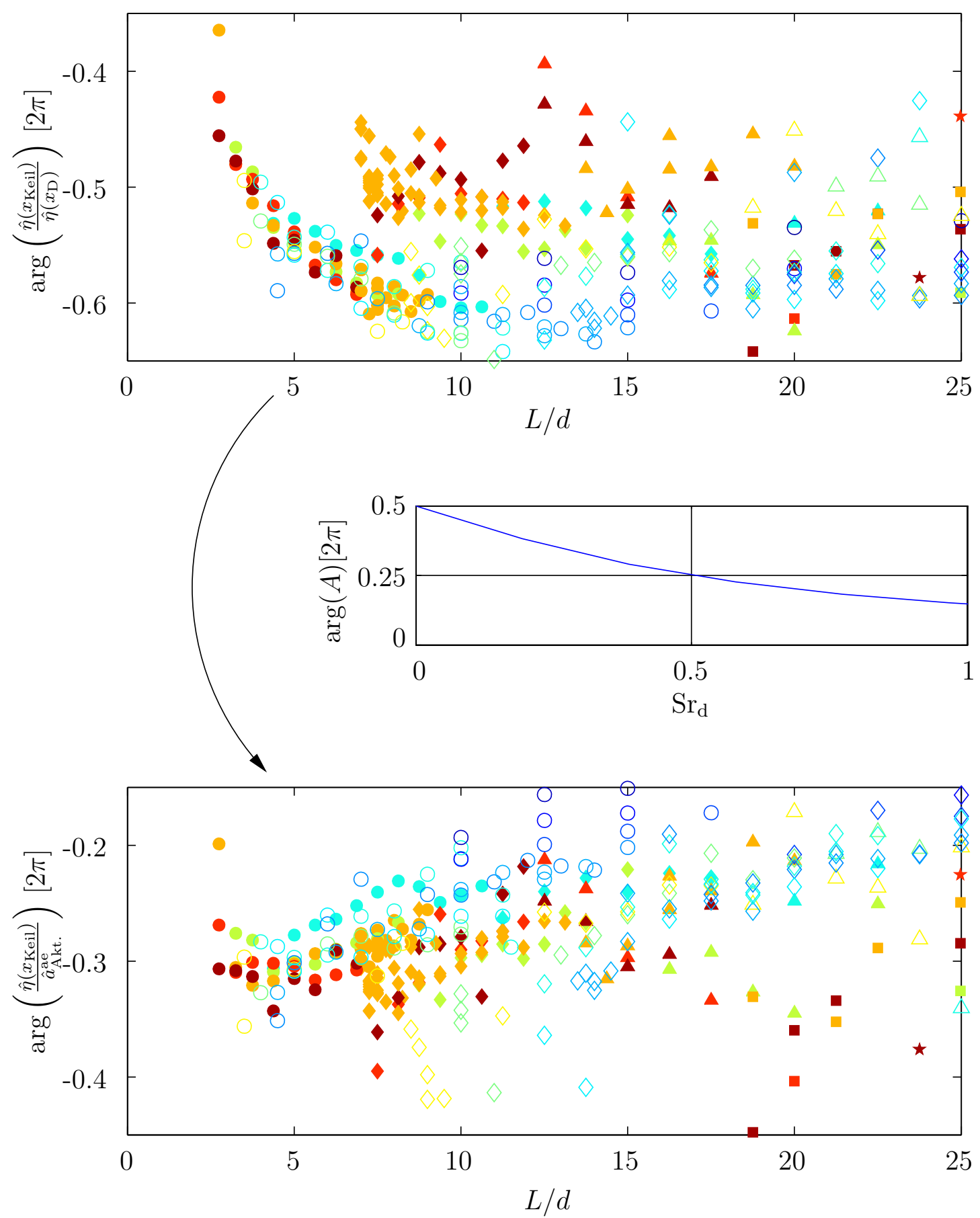

Abb. 4.4: Phasenbeziehungen zwischen Düse und Keil. Oben: Phase der Strahl-Übertragungsfunktion (Phase modulo $2 \pi$ aus Abb. 4.3), Mitte: Phase der Strahlrezeptivität, unten: Phase der Übertragungsfunktion der äquivalenten Aktuatorauslenkung zur Auslenkung am Keil. Legende siehe Abb. 4.3. 


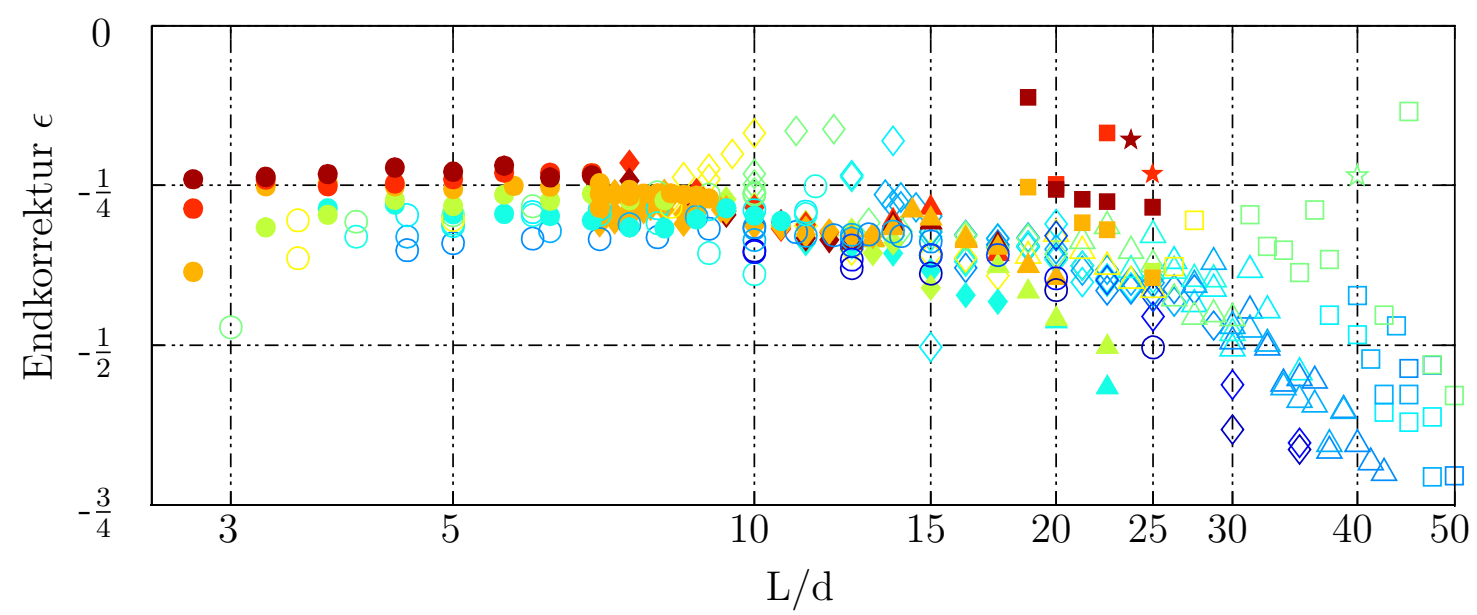

Abb. 4.5: Endkorrektur der Moden. $\diamond$ Mode I, $\diamond$ Mode II, $\triangle$ Mode III, $\square$ Mode IV,$\star$ Mode V ; gefüllte Symbole $d=4 \mathrm{~mm}$, sonst $d=2 \mathrm{~mm}$; Farbe kodiert von blau nach rot die Reynoldszahl (Abb. 4.6).

im zugrundeliegenden Strömungsfeld einstellt und nicht in der Streichlinienauslenkung. Daher wird vermutet, dass zwei ähnliche, aber entgegengesetzte Prozesse (vergleichbar mit der Strahlrezeptivität) an Düse und Keil stattfinden.

\subsubsection{Phasendrehung bei der Instabilitätswellenausbreitung}

Die Phasendrehung aufgrund der Instabilitätswellenausbreitung kann durch den mittleren komplexen Phasengradienten berechnet werden, der nur an den Stromab-Positionen $x_{1}<x<x_{2}$ in Abb. 4.2 ausgewertet wurde, an denen er etwa konstant verläuft. Die Phasendrehung der Instabilitätswelle zwischen Düse und Keil kann daraus extrapoliert werden.

Betrachtet man die aus der Instabilitätswelle resultierende Phasendrehung von Düse zu Keil

$$
\Delta \varphi_{\text {Inst. }}=L \cdot\left\langle\frac{\partial \varphi_{\text {Inst. }}}{\partial x}\right\rangle_{x_{\mathrm{D}}<x<x_{\text {Keil }}} \approx-L \cdot\left\langle\arg \left\{\hat{\eta}(x) / \hat{\eta}\left(x_{\mathrm{D}}\right)\right\}\right\rangle_{x_{1}<x<x_{2}},
$$

so kann man fragen, welche Phasendifferenz $\epsilon$ in Einheiten von $2 \pi$ an der Erfüllung der Schleifenbedingung $-2 \pi \cdot m$ fehlen:

$$
2 \pi \cdot \epsilon=-2 \pi m+L \cdot\left\langle\frac{\partial \varphi_{\text {Inst. }}}{\partial x}\right\rangle_{x_{\mathrm{D}}<x<x_{\text {Keil }}} .
$$

Dabei ist $\epsilon$ die sogenannte Endkorrektur, die u.a. bei Powell [37], Crighton [8], Maurel [27] und Howe [20] diskutiert wird. Ein negatives $\epsilon$ stellt eine Phasenverzögerung dar. Diese restliche Phasendrehung $\epsilon \cdot 2 \pi$ muss durch andere Prozesse, wie die Strahl-Kanten-Wechselwirkung, die Anregung der Instabilitätswelle oder verlängerte/verkürzte Laufwege erklärt werden. 
Kapitel 4. Struktur und Dynamik der Moden

\begin{tabular}{|c|c|c|c|c|}
\hline Koeffizient & $c_{\mathrm{Re}_{\mathrm{d}}}$ & $c_{\mathrm{L} / \mathrm{d}}$ & $c_{\mathrm{d}}$ & $c_{\mathrm{m}}$ \\
\hline Wert & $4 \cdot 10^{-4}$ & $-9 \cdot 10^{-3}$ & $-0.2 / \mathrm{cm}$ & 0.03 \\
auf Streuung normiert & 0.23 & -1.02 & -0.23 & 0.30 \\
\hline
\end{tabular}

Tabelle 4.1: Regressionstabelle für die Endkorrektur

Die Endkorrektur ist dabei nichts anderes als der Achsenabschnitt nach einer Extrapolation der in Abb. 4.2 gezeigten Phasen nach $x / L=0$ und einer Verschiebung der Kurven um circa $\pi$ nach unten, so dass sie bei $x / L=1$ ein Vielfaches von $-2 \pi$ erreichen. Die Endkorrekturwerte sind für sehr kleine Keilabstände wegen der zusammenwachsenden Nahfelder an Düse und Keil nicht mit Sicherheit bestimmbar. Ebenso wird die Bestimmung von $\epsilon$ durch den stromab beschränkten Messbereich ungenauer, da die Phase sehr weit extrapoliert werden muss und somit der stromab größer werdende Betrag des Phasengradienten nicht angemessen berücksichtigt wird. Daher ist für große Keilabstände die Endkorrektur zu groß bestimmt, was auch Abb. 4.5 vermuten lässt. Um die Abhängigkeit der räumlichen Struktur von der gewählten Düsenbreite und damit von der Kanalgeometrie zu untersuchen, wird hier eine Regressionsanalyse mit dem Modell

$$
\epsilon=c_{\mathrm{Re}_{\mathrm{d}}} \mathrm{Re}_{\mathrm{d}}+\mathrm{c}_{\mathrm{L} / \mathrm{d}} \mathrm{L} / \mathrm{d}+\mathrm{c}_{\mathrm{d}} \mathrm{d}+\mathrm{c}_{\mathrm{m}} \quad \text { m mit } \epsilon=(\mathrm{L} / \lambda-\mathrm{m})
$$

durchgeführt. Das Modell kann die Varianz der Endkorrektur um circa 40\% vermindern. Dabei werden nur solche Reynoldszahlen berücksichtigt, die Daten von beiden Düsen beinhalten. Der Parameter Keilabstand $L / d$ hat den größten standardisierten Regressionskoeffizienten. Die restlichen drei Parameter gehen etwa mit gleichem Gewicht ein (siehe Tab. 4.1). Mit größerem Keilabstand und größerer Düsenbreite wird die Endkorrektur betragsmäßig größer, während sie mit steigender Reynoldszahl und steigender Modennummer sinkt. Damit lässt die Untersuchung den Schluss zu, dass die Kanaltiefe $B$ kaum einen Effekt auf die Varianz der Endkorrektur hat, da sonst eine größere Abhängigkeit der Endkorrektur von der Düsenbreite zu erwarten gewesen wäre.

\subsection{Zusammenhang zwischen räumlicher und zeitlicher Struktur: Modenspektrum}

Die im letzten Abschnitt untersuchte räumliche Phasenänderung $\frac{\partial \varphi_{\text {Inst. }}}{\partial x}$ soll nun mit der gemessenen zeitlichen Änderung $\frac{\partial \varphi_{\text {Inst. }}}{\partial t}=\omega_{\mathrm{m}}$ in Verbindung gebracht werden. Die Frequenz $\omega_{\mathrm{m}}$, auf der das System selbsterregt schwingt, hängt für eine bestimmte Mode $m$ in guter Näherung von der leicht korrigierten Phasenlaufzeit der Instabilitätswelle zwischen Düse und Keil ab.

Setzt man nach Gl. (4.6) die Gesamtphasendrehung für $m$ Umläufe durch die Phasendrehung der Instabilitätswelle mit der Korrektur $\epsilon$ an, kann die Strouhalzahl bezüglich der Durchfluss- 


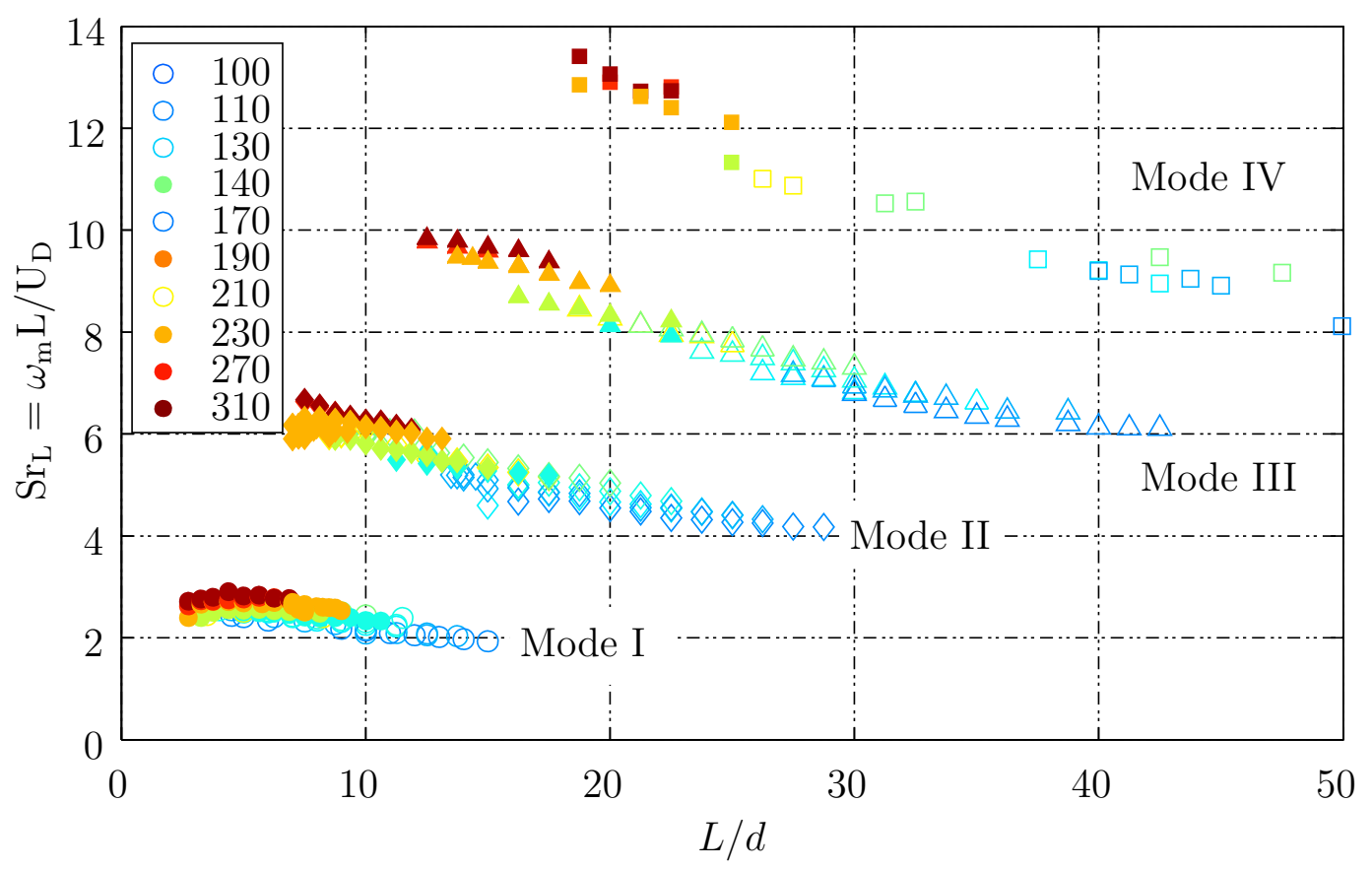

Abb. 4.6: Strouhalzahl der natürlichen Moden in Abhängigkeit des Düse-KeilAbstandes für verschiedene Moden und Reynoldszahlen. o Mode I, $\diamond$ Mode II, $\triangle$ Mode III, $\square$ Mode IV; gefüllte Symbole $d=4 \mathrm{~mm}$, sonst $d=2 \mathrm{~mm}$; die Farbe kodiert die Reynoldszahl von blau nach rot.

geschwindigkeit $U_{\mathrm{D}}$ durch

$$
\mathrm{Sr}_{\mathrm{L}}=\frac{\omega_{\mathrm{m}} \mathrm{L}}{\mathrm{U}_{\mathrm{D}}}=\frac{\omega_{\mathrm{m}}(\mathrm{m}+\epsilon) 2 \pi}{\mathrm{U}_{\mathrm{D}}\left\langle\frac{\partial \varphi_{\text {Inst. }}}{\partial \mathrm{x}}\right\rangle_{\mathrm{x}_{\mathrm{D}}<\mathrm{x}<\mathrm{x}_{\text {Keil }}}}
$$

ausgedrückt werden.

Das Modenspektrum der nach Abschnitt 4.2 ausgewählten Peakfrequenzen $\omega_{\mathrm{m}}$ ist in Abb. $4.6 \mathrm{zu}$ sehen. Zum einen sinkt die Strouhalzahl mit größer werdendem normierten Keilabstand stark ab und zum anderen steigt sie mit größerer Reynoldszahl leicht an. Für feste Reynoldszahlen liegen die Strouhalzahlen der Moden nicht ganzzahlig zueinander, da der Phasengradient der Instabilitätswelle von der Frequenz und der Instabilitätswellenamplitude selbst abhängt und für verschiedene Frequenzen auch unterschiedliche Phasendrehungen durch die Strahl-KantenWechselwirkung und die Anregung der Instabilitätswelle entstehen können.

\subsubsection{Absinken der Strouhalzahl mit dem Düse-Keil-Abstand}

Von Crigthon [8] (siehe auch Howe [20]) wird die Strouhalzahl aus der leicht korrigierten Phasenlaufzeit und bestimmten Annahmen über die Rezeptivität an Düse und Keil berechnet. 
Kapitel 4. Struktur und Dynamik der Moden

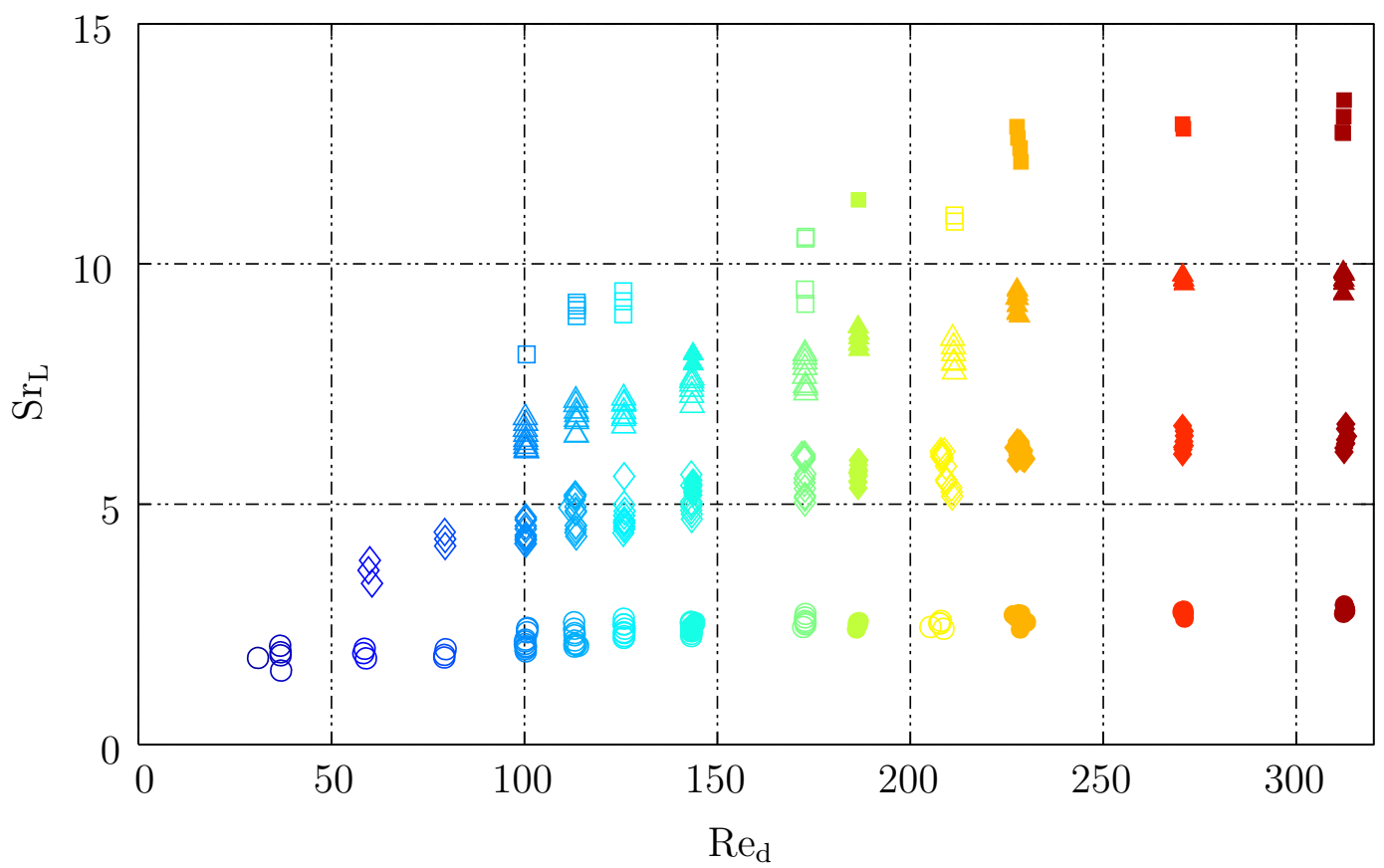

Abb. 4.7: Strouhalzahl in Abhängigkeit der Reynoldszahl. Legende wie in Abb. 4.6.

Für $L \gg d$ ergibt sich eine Abhängigkeit der Form

$$
\mathrm{Sr}_{\mathrm{L}} \propto(\mathrm{L} / \mathrm{d})^{(-1 / 2)} .
$$

Hier findet man Abhängigkeiten zwischen $L^{-0.05 \cdots-0.45}$. Für größere $L / d$ und somit höhere Moden nähert sich die Potenz dem Wert -0.5 ausgehend von etwa -0.05 bei Mode I bis auf etwa -0.45 bei Mode IV.

Die Phasendrehung pro Laufweg $\frac{\partial \varphi_{\text {Inst. }}}{\partial x}$ steigt mit $L / d$ an (Abb. 3.5), und somit fällt $\operatorname{Sr}_{\mathrm{L}}$ entsprechend mit $L / d$ ab (siehe Gl. (4.8)). Jedoch ist die verlängerte Laufzeit nicht der alleinige Grund dafür, denn die Endkorrektur $\epsilon$ hängt in dem Modell gemäß Gl. (4.8) ebenfalls systematisch von $L / d$ ab (wie in Tabelle 4.1 gezeigt) und sinkt ebenfalls mit steigendem $L / d$. Da sowohl $\frac{\partial \varphi_{\text {Inst. }}}{\partial x}$ als auch die zusätzliche Phasendrehungen durch die Strahl-Kanten-Wechselwirkung und die Anregung der Instabilitätswelle (beschrieben durch $\epsilon$ ) von der Auslenkungsamplitude abhängen, lassen sich die beiden Anteile nicht trennen. Letztendlich ist damit die Frequenz $\omega_{\mathrm{m}}$ in schwer beschreibbarer Weise von der räumlichen Verteilung der Auslenkungsamplitude abhängig, die in Abschnitt 4.5 untersucht wird.

\subsubsection{Anstieg der Strouhalzahl mit der Reynoldszahl}

Die Strouhalzahl $\mathrm{Sr}_{\mathrm{L}}$ steigt mit der Reynoldszahl leicht an (siehe Abb. 4.7). Dies wird plausibel, wenn man die Entwicklung des $\bar{U}$-Profils in Abhängigkeit von der Reynoldszahl betrachtet. Wie 


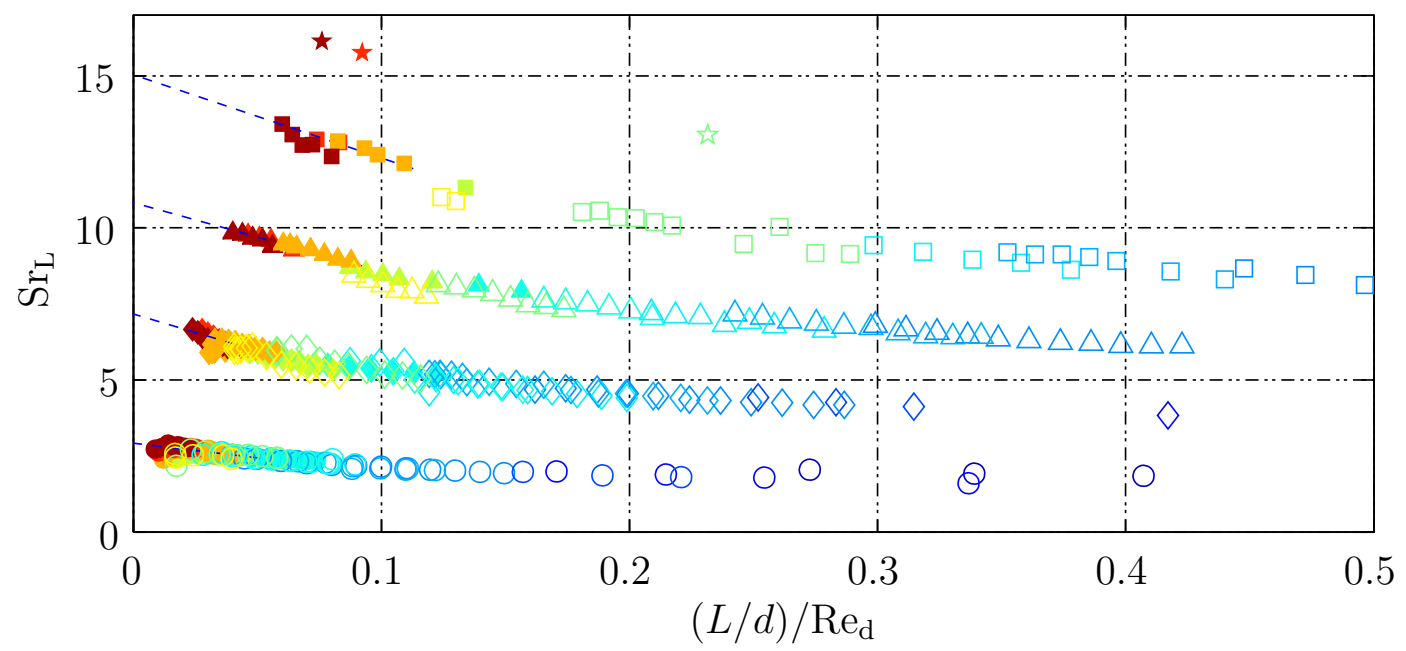

Abb. 4.8: Strouhalzahl in Abhängigkeit von $(\boldsymbol{L} / \boldsymbol{d}) / \mathbf{R e}_{\mathbf{d}}$. Legende wie in Abb. 4.6.

bei der Plattengrenzschicht kann man sich eine Reynoldszahl mit der Lauflänge gebildet denken, so dass der Ort, an dem sich ein bestimmtes Strömungsprofil eingestellt hat, bei Erhöhung der Strömungsgeschwindigkeit stromab wandert. Betrachtet man einen festen Ort und erhöht $\mathrm{Re}_{\mathrm{d}}$, so hat sich das Profil durch die viskosen Schubspannungen noch nicht so stark verbreitert, damit verringert sich $\left\langle\frac{\partial \varphi_{\text {Inst. }}}{\partial x}\right\rangle$ und $\mathrm{Sr}_{\mathrm{L}}$ steigt mit zunehmender $\mathrm{Re}_{\mathrm{d}}$ an. Da sich mit Erhöhung der Strömungsgeschwindigkeit das Profil ändert und damit auch die Instabilitätswelle, die wiederum selbst auf das Strömungsprofil rückwirkt, ist der Prozess $\operatorname{Re}_{\mathrm{d}} \rightarrow \infty$ kompliziert. Die Strouhalzahl sollte jedoch für $\mathrm{Re}_{\mathrm{d}} \rightarrow \infty$ gegen die konstante Strouhalzahl $\mathrm{Sr}_{\mathrm{L} \infty}$ gehen (RAGHU \& Monkewitz [40] in Zusammenhang mit dem „vortex shedding“). Trägt man $\operatorname{Sr}_{\mathrm{L}}$ gegen $\frac{L}{d \cdot \operatorname{Re}_{\mathrm{d}}}$ auf (Abb. 4.8), so ergeben sich abfallende Kurven, die für kleine $(L / d) / \operatorname{Re}_{\mathrm{d}}$ als linear angesehen werden können und die $y$-Achse bei $\mathrm{Sr}_{\mathrm{L} \infty}$ schneiden.

\subsection{Räumliche Amplitudenentwicklung der Moden}

Die eben diskutierte Reynoldszahlabhängigkeit der Strouhalzahl wird im Wesentlichen durch die Amplitudenentwicklung der Instabilitätswelle mit dem Laufweg bestimmt. Die jeweils am Keil erreichte Endamplitude der Moden nimmt etwa linear mit $L / d$ zu (Abb. 4.9), wobei ein geringfügiger, aber signifikanter Anstieg mit der Reynoldszahl zu beobachten ist, der bei kleiner Reynoldszahl in der Nähe der Schwelle besonders ausgeprägt ist.

Die eingeschwungene Mode hat die Gesamtschleifenverstärkung 1. Die konvektive Verstärkung der Instabilitätswelle muss den kleinen Übertragungsfaktor $(-25 \mathrm{~dB}$ bis $-70 \mathrm{~dB})$ der Strahl- 
Kapitel 4. Struktur und Dynamik der Moden

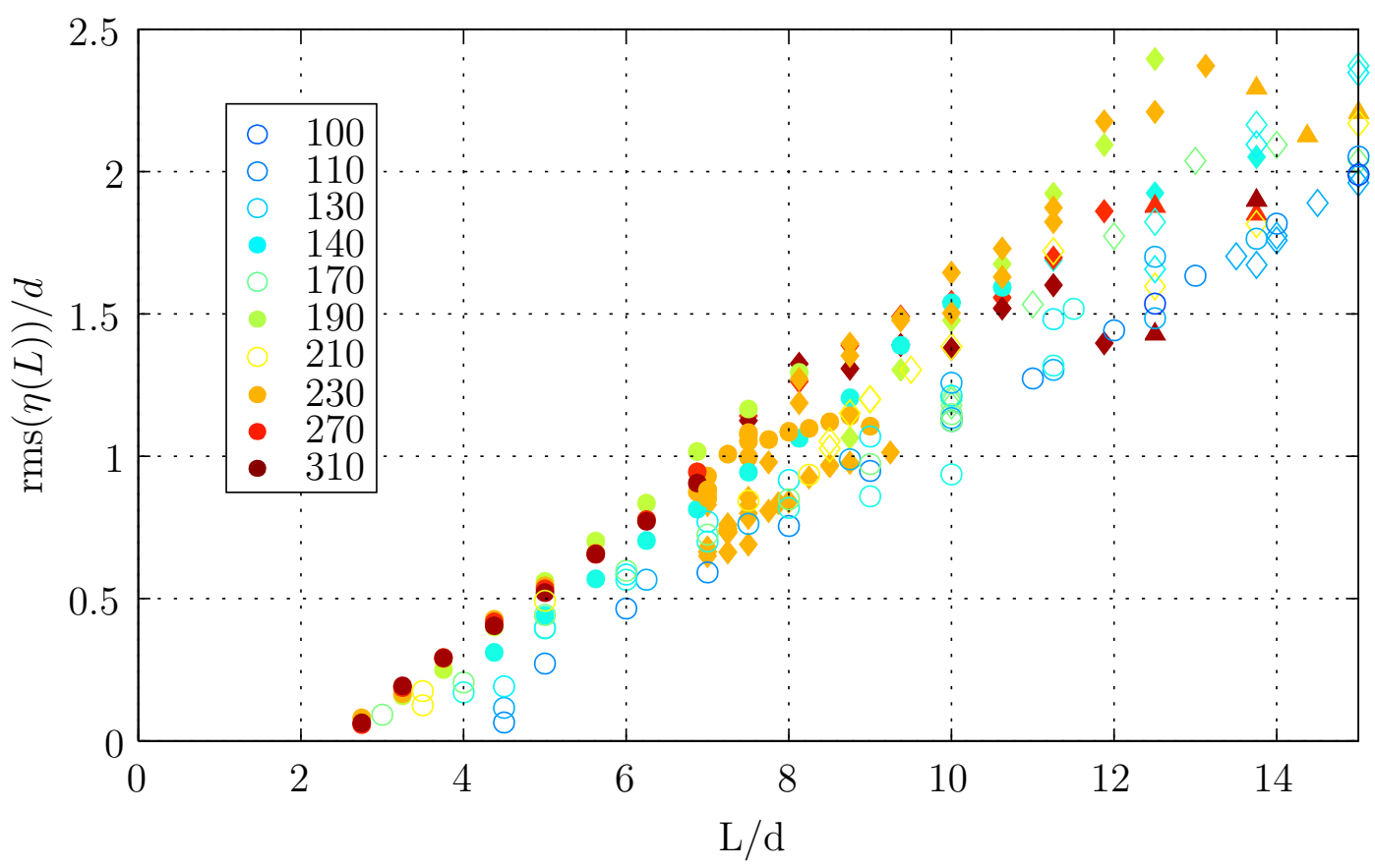

Abb. 4.9: Erreichte Endamplitude der selbsterregten Schwingung als Funktion des normierten Keilabstands. Legende siehe Abb. 4.5.

Kanten-Wechselwirkung kompensieren. Dies geschieht für $L \gtrsim 2.5 d$. Für größere Keilabstände ist das konvektive Verstärkungspotenzial des Freistrahls immer deutlich größer als der Abfall durch die rückkoppelnde Strahl-Kanten-Wechselwirkung.

Der Freistrahl wird nun - so die Modellvorstellung - durch die entstehenden Wechselschubspannungen verbreitert. Je größer die Amplitude der Instabilitätswelle ist, desto breiter wird das aufgrund der Reynoldschen Schubspannungen entstehende mittlere Gleichströmungsprofil. Die auf das lokale Strömungsprofil bezogene Strouhalzahl verschiebt sich mit wachsender lokaler Instabilitätswellenamplitude zu immer höheren Strouhalzahlen. Dabei nimmt der Imaginärteil der Wellenzahl, entsprechend der Dispersionsrelation für das lokale Strömungsprofil $\mathfrak{I m}\left\{k\left(x,\left|\tilde{\eta}_{\text {Inst. }}(x)\right|, \omega_{\mathrm{m}}\right)\right\}$, oberhalb einer bestimmten Strouhalzahl als Funktion der Instabilitätswellenamplitude $\left|\tilde{\eta}_{\text {Inst. }}\right|$ immer stärker ab und damit auch die gesamte konvektive Verstärkung des Strahls. Insgesamt lässt sich die Amplitudenbedingung der Rückkopplungsschleife formulieren durch:

$$
\begin{aligned}
& \left|\tilde{\eta}_{\text {Inst. }}\left(x_{\text {Keil }}\right)\right|=\left|\tilde{\eta}_{\text {Inst. }}\left(x_{\mathrm{D}}\right)\right| \cdot e^{\int_{x_{\mathrm{D}}}^{x_{\text {Keil }}} \mathfrak{I m}\left\{k\left(x,\left|\tilde{\eta}_{\text {Inst. }}(x)\right|, \omega_{\mathrm{m}}\right)\right\} d x}
\end{aligned}
$$

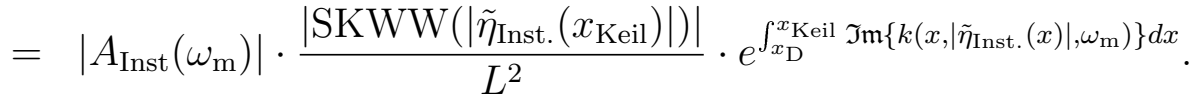

Dabei symbolisiert SKWW $/ L^{2}$ die Umsetzung von $\tilde{\eta}\left(x_{\text {Keil }}\right)$ auf die Auslenkungsamplitude $\hat{a}_{\text {Akt. }}^{\text {ae }}$ eines gedachten Aktuators, der genau die gleiche Wirkung auf die Anregung der Instabilitäts- 
welle hat wie die Strahl-Kanten-Wechselwirkung. Da die Strahl-Kanten-Wechselwirkung durch ein Druckdipolfeld auf die Düse übertragen wird, fällt der Übertragungsfaktor zur Düse mit $1 / L^{2}$ ab. Die Anregung der Instabilitätswelle wird durch die Übertragungsfunktion $A_{\text {Inst }}\left(\omega_{\mathrm{m}}\right)$ von der Aktuatorauslenkung $\hat{a}_{\text {Akt. }}^{\text {ae }}$ auf die Instabilitätswellenauslenkung $\hat{\eta}_{\text {Inst. }}\left(x_{\mathrm{D}}\right)$ beschrieben. Die Strahl-Kanten-Wechselwirkung steht in einem noch unbekannten Zusammenhang mit der Endamplitude am Keil $\left|\tilde{\eta}_{\text {Inst. }}\left(x_{\text {Keil }}\right)\right|$ oder einer anderen Strömungsgröße, die dann wahrscheinlich proportional zu $\left|\tilde{\eta}_{\text {Inst. }}\left(x_{\text {Keil }}\right)\right|$ ist. Die Strahl-Kanten-Wechselwirkung ist nichtlinear mit der Auslenkungsamplitude am Keil verknüpft. Man kann sich vorstellen, dass der Linearitätsbereich aufhört, wenn der Strahl soweit ausgelenkt wird, dass selbst der Strahlrand nicht mehr in direktem Kontakt mit dem Keil steht. Dann stehen nur noch die durch den oszillierenden Strahl erzeugten Druckfelder mit dem Keil in Wechselwirkung. Dies entspräche einem Keilabstand von ungefähr $7 L / d$, wenn man die Verbreiterung des Strömungsprofils stromab außer Acht lässt und von einer durchschnittlichen Strahlauslenkung von $0.15 \mathrm{~L}$ ausgeht. Gehen die Auslenkungsamplituden bzw. der Keilabstand darüber hinaus, so ist eine Abnahme der StrahlKanten-Wechselwirkung zu erwarten.

Um die in Abb. 4.9 gezeigte lineare Zunahme der am Keil erreichten Streichlinienauslenkung mit dem Düse-Keil-Abstand verständlich zu machen, kann man davon ausgehen, dass die potentielle Strahlverstärkung die Keilrückkopplung stark überkompensiert. Dann muss die Strahlbreite stark zunehmen, damit im eingeschwungenen Zustand die Schleifenverstärkung auf den Wert 1 reduziert wird. Man kann dann erwarten, dass der Strahl am Keil eine so große Breite $d_{\text {Strahl,end }}$ erreicht hat, dass die mit $d_{\text {Strahl,end }}$ gebildete Strouhalzahl nahezu den Grenzwert erreicht, bei dem die konvektive Verstärkung null wird. Nimmt man nun vereinfachend an, dass die am Keil erreichte Strouhalzahl $\mathrm{Sr}_{\text {end }}=\omega_{\mathrm{m}} d_{\text {Strahl,end }} / \bar{U}$ tatsächlich unabhängig von $L / d$ ist und geht weiter davon aus, dass $\omega_{\mathrm{m}} \propto \bar{U} / L$ ist, so folgt

$$
d_{\text {Strahl }, \text { end }}=\frac{\mathrm{Sr}_{\text {end }} \bar{U}}{\omega_{\mathrm{m}}} \propto \frac{\mathrm{Sr}_{\text {end }}}{L}
$$

und schließlich mit der für große Strahlverbreiterungen plausiblen Annahme $d_{\text {Strahl,end }} \propto$ $\hat{\eta}_{\text {Inst. }}\left(x_{\text {Keil }}\right)$

$$
\hat{\eta}_{\text {Inst. }}\left(x_{\text {Keil }}\right) \propto L .
$$

Diese Betrachtung legt nahe, dass die gesamte Strömung im hinteren, keilnahen Bereich nur noch mit $L$ skaliert und nur noch abhängig von der Mode $m$ ist, in der der Strahl schwingt. Die obige Annahme, dass $d_{\text {Strahl,end }} \propto \hat{\eta}_{\text {Inst. }}\left(x_{\text {Keil }}\right)$ kann mit dem einfachen Modell aus Kap. 3.5.1 und Kap. B.1.2 allerdings nicht direkt in allen Einzelheiten bestätigt werden, da die $\bar{U}(y)$ Profile bei Verbreiterung durch die Faltung mit der Amplitudenverteilung der Instabilitätswellenauslenkung nicht selbstähnlich bleiben. In Abb. 4.10 sind die erreichten Endamplituden $\hat{\eta}\left(x_{\text {Keil }}\right) \approx \hat{\eta}_{\text {Inst. }}\left(x_{\text {Keil }}\right)$ einiger Moden zuzüglich einer Korrektur von $0.3 d$ aufgetragen (d.h. $\left.\hat{\eta}\left(x_{\text {Keil }}\right)+0.3 \propto L\right)$. Diese Korrektur zeigt, dass erst für große Keilabstände der Einfluss der Düse auf die erreichte Endamplitude vernachlässigbar wird. 

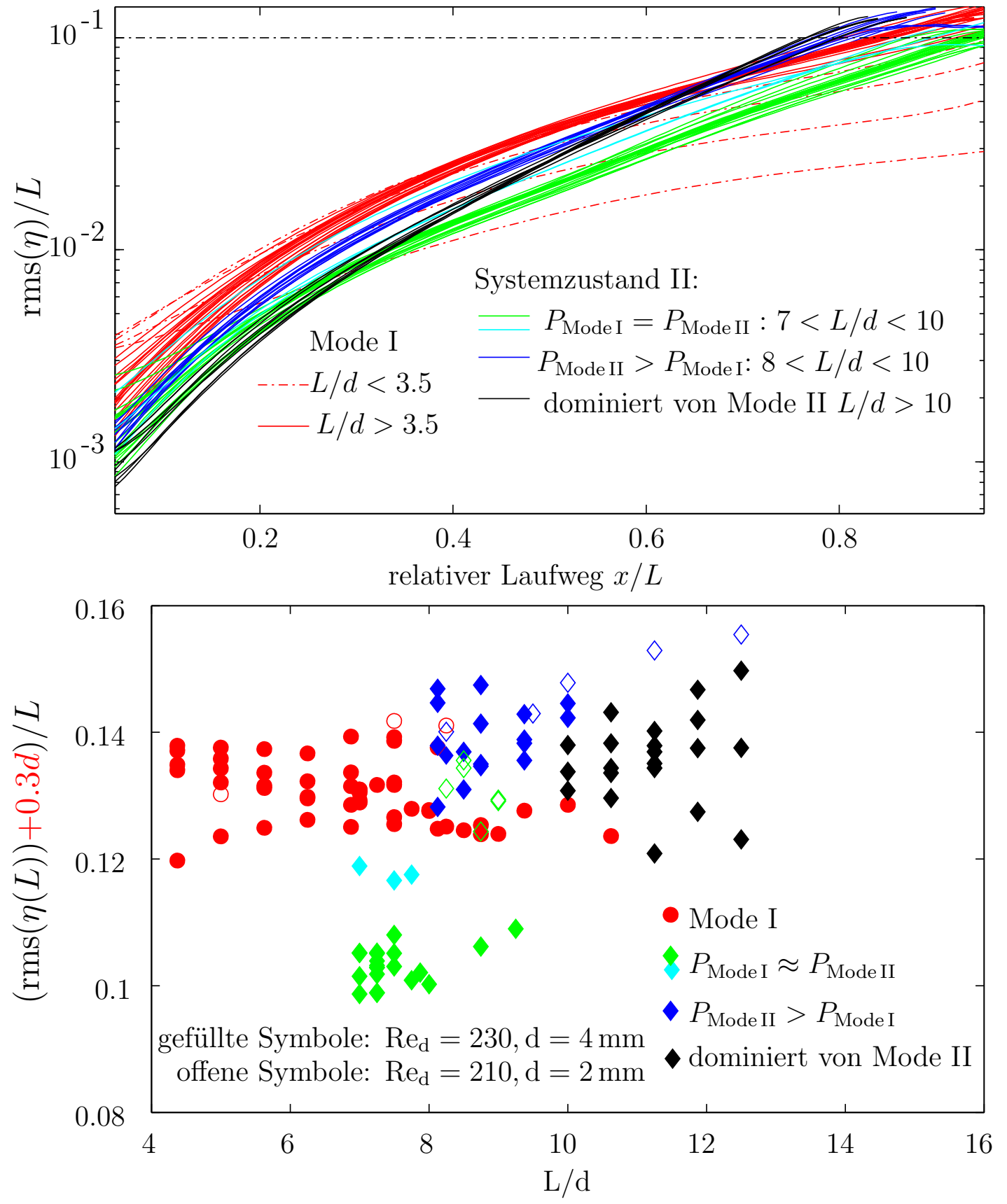

Abb. 4.10: Amplitudenverhalten von Moden und Modengemischen. Oben: Amplitudenentwicklung der selbsterregten Schwingung. Der rms-Wert von $\eta$ wurde dabei auf $L$ normiert. Unten: Der auf $x_{\text {Keil }}$ extrapolierte rms-Wert von $\eta$ zuzüglich $0.3 d$ auf $L$ normiert. 
Wie im nächsten Abschnitt ausführlich diskutiert wird, ist in Gegenwart einer höheren Mode auch immer eine leicht modifizierte tiefere Mode anwesend. Dabei konkurrieren die verschiedenen Moden um das begrenzte Verstärkungspotenzial der Freistrahlinstabilität. Der rms-Wert der Streichlinienauslenkung zeigt für jeden Systemzustand $m$, der sich aus den Moden $1 \ldots m$ zusammensetzt, eine charakteristische Abhängigkeit vom Laufweg. In Abb. 4.10 (oben) unterscheidet sich die Amplitudenabhängigkeit des Systemzustands II, wenn sich zwei Moden (Peaks im Frequenzspektrum) mit ähnlicher Leistung überlagern (grüne Kurven), von der mit einer dominierenden Mode (blaue und schwarze Kurven, wobei die blauen Kurven noch größere Anteile der Mode I enthalten und die schwarzen von Mode II dominiert werden). Die erreichten Endamplituden am Keil sind bei den Systemzuständen mit zwei etwa leistungsgleichen Peaks geringer (Abb. 4.10 (unten)). Die konvektive Verstärkung des Freistrahls wird offensichtlich durch das Modengemisch zweier ähnlich stark vertretener Moden stärker herabgesetzt als durch ein Gemisch mit einer dominanten Mode.

\subsection{Hysterese und Systemzustände aus mehreren Mo- den}

In Abbildung 4.11 sind Bilder zweier Systemzustände zu sehen, die bei gleichem Keilabstand und gleicher Reynoldszahl möglich sind. Vergrößert man den Abstand zwischen Düse und Keil, so stellt sich oberhalb eines gewissen Abstands der Strömungszustand um, und eine höhere Mode wird destabilisiert. Verkleinert man den Abstand wieder, so bleibt der eingestellte Strömungszustand auch zu kleineren Düse-Keil-Abständen erhalten. Daher gibt es einen Bereich von Keilabständen, in dem zwei Systemzustände existieren können. Die Größe dieses Hysteresebereichs hängt sehr stark von den Fluktuationen der Strömung ab. Das Gebiet wird umso größer, je länger der vorhergehende Systemzustand gedauert hat und je weniger Störungen man durch das Verschieben des Keils einbringt. Das System reagiert vor allem an den Rändern des Hysteresebereichs äußerst empfindlich auf hohe eingestreute Frequenzen, die das System mit großer Anwachsrate in einen anderen Systemzustand übergehen lassen.

In Abb. 4.12 sind für $\mathrm{Re}_{\mathrm{d}}=230$ die Hysteresebereiche eingezeichnet, die man durch vorsichtiges Verstellen des Keils erhält. Dabei wurde der Übergang zwischen Mode I und Mode II besonders sorgfältig untersucht. Der Hysteresebereich erstreckt sich typischerweise über $\Delta L / d=2$.

An den Rändern des Hysteresebereiches lassen sich spontane Wechsel zwischen den System-

zuständen beobachten, ohne dass das System von außen aktiv beeinflusst wird. Am linken Rand kann der höhere Systemzustand spontan in den nächst niedrigeren übergehen und am rechten Rand der niedrigere in den höheren; dabei sind die Randgebiete circa $0.5 d$ breit. Gerade nach einer aktiven Stabilisierung (Kapitel 5) kommt es vor, dass das System auf dem höheren Systemzustand anschwingt und nach einer gewissen Verweildauer in den niedrigeren zurückfällt. Dabei kann die Zeit, die das System braucht um spontan in einen anderen Zustand überzuge- 

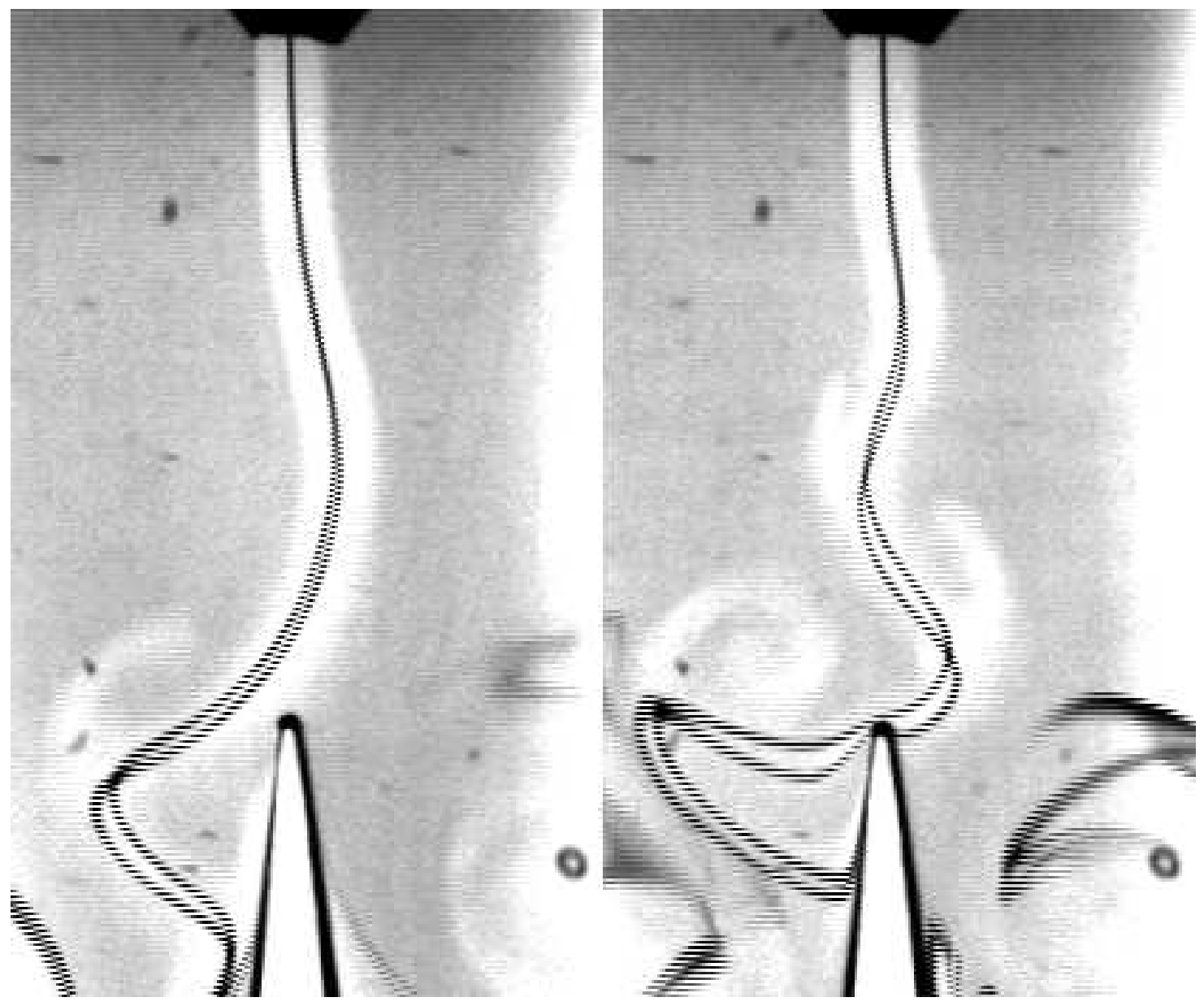

Abb. 4.11: Bilder zweier verschiedener Systemzustände bei gleichem Keilabstand $10 L / d$, $d=2 \mathrm{~mm}, \mathrm{Re}_{\mathrm{d}}=181$. Die geraden und die ungeraden Zeilen des Videobildes sind um $20 \mathrm{~ms}$ versetzt, daher erscheint die Streichlinie in Gebieten mit großer Änderung der Streichlinie doppelt.

hen, sehr groß im Vergleich zu der Schwingungsdauer des Systems sein. In Abb. 4.13 ist das Spektrogramm eines solchen spontanen Übergangs vom Systemzustand II zu Mode I zu sehen. Die Verweildauer von 340 Sekunden ist dabei nicht untypisch. Erhöht man den Düse-KeilAbstand, so wechselt das System auf den höheren Systemzustand zurück; wartet man bis der höhere Systemzustand stabil eingeschwungen ist und zieht den Keil wieder auf die alte Position zurück, so stellt sich eine ähnliche Verweildauer ein wie beim vorhergehenden Mal. Damit kann dieser Effekt nicht auf langsame Fluktuationen der Systemparameter zurückgeführt werden. Man kann zwei verschiedene Sichtweisen entwickeln: Entweder führen die zufälligen Flukuationen der Strömungsparameter durch eine günstige Koinzidenz zu dem Zustandswechsel, oder das System kann als autonom betrachtet werden, wobei die Trajektorie des Systems nach einer gewissen Zeit ein Gebiet im Phasenraum durchläuft, in dem das System den anderen Zustand 


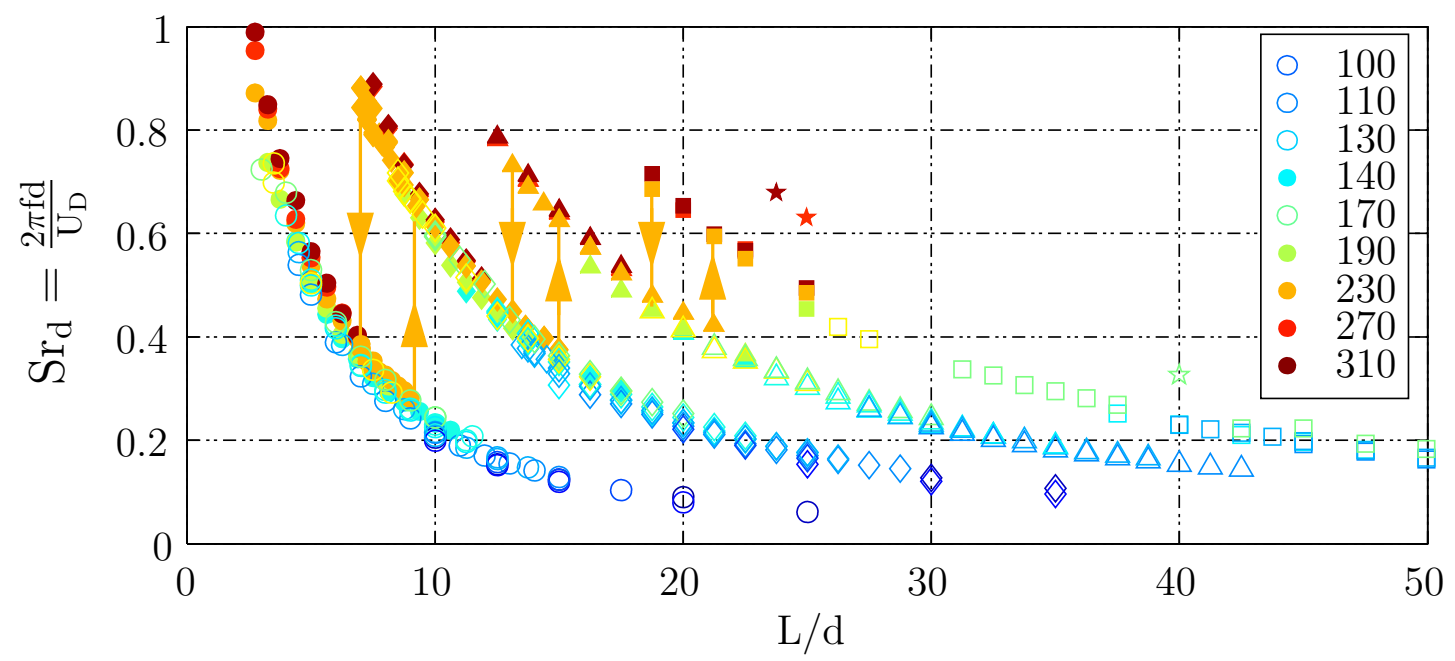

Abb. 4.12: $\mathbf{S r}_{\mathbf{d}}$ gegen $\boldsymbol{L} / \boldsymbol{d}$. Die Hysterese ist für $\mathrm{Re}_{\mathrm{d}}=230$ eingezeichnet, die Messdaten repäsentieren hier nicht das vollständige Modenspektrum bezüglich der für eine Mode realisierbaren $L / d$.

bevorzugt. Dies erfordert allerdings einen nicht periodischen Anteil in der Trajektorie, der hier für höhere Systemzustände auch vorhanden ist (siehe Abschnitt 4.7). Im ersteren Falle wäre die Verweildauer von den Parameterfluktuationen, wie sie beispielsweise durch die Konvektion oder die Wirbelbildung im Einlauf herrühren, abhängig. Im anderen Fall braucht das System eine mittlere Zeit, bis es das entsprechende Gebiet im Phasenraum gefunden hat. Experimente haben gezeigt, dass die Verweildauer des Systems durch Beruhigung des Einlaufs oder längerer aktiver Stabilisierung erhöht werden kann.

Im Allgemeinen sind die verschiedenen Systemzustände durch die Hauptfrequenzpeaks im Leistungsspektrum und die dazugehörigen räumlichen Schwingungsformen charakterisiert. Für die Moden bei kleinen Düse-Keil-Abständen findet man stets einen markanten Peak im Leistungsspektrum mit seinen ungeraden Harmonischen, so dass man dort von einer Mode sprechen kann. In Abb. 4.13 ist die Mode I für $t>340$ s präsent. Der nächst höhere Systemzustand $(t<340$ s $)$ ist durch zwei nicht harmonisch liegende Frequenzpeaks mit einem Frequenzverhältnis von 2.83 gekennzeichnet. Dabei sind die spektralen Leistungsdichten bei der niederfrequenteren Peak-Frequenz $f^{\mathrm{T}}$ und der höherfrequenteren Peak-Frequenz $f^{\mathrm{H}}$ etwa gleich. Die Zeitreihe in Abb. 4.13 ist daher zunächst eine Überlagerung dieser zwei nicht harmonischen Frequenzen $f^{\mathrm{H}}$ und $f^{\mathrm{T}}$. Es liegt nahe, jedem markanten Peak des Leistungsspektrums eine eigene Mode zuzuordnen, so dass der Systemzustand eine Überlagerung von Moden ist, der durch die höchste darin enthaltene Mode charakterisiert ist.

Diese Koexistenz von Moden wirft allerdings die Frage auf, ob und wie stark sich die einzelnen Moden beeinflussen (siehe Abschnitt 4.7). Die Lage und die spektrale Leistung der Spitzen- 


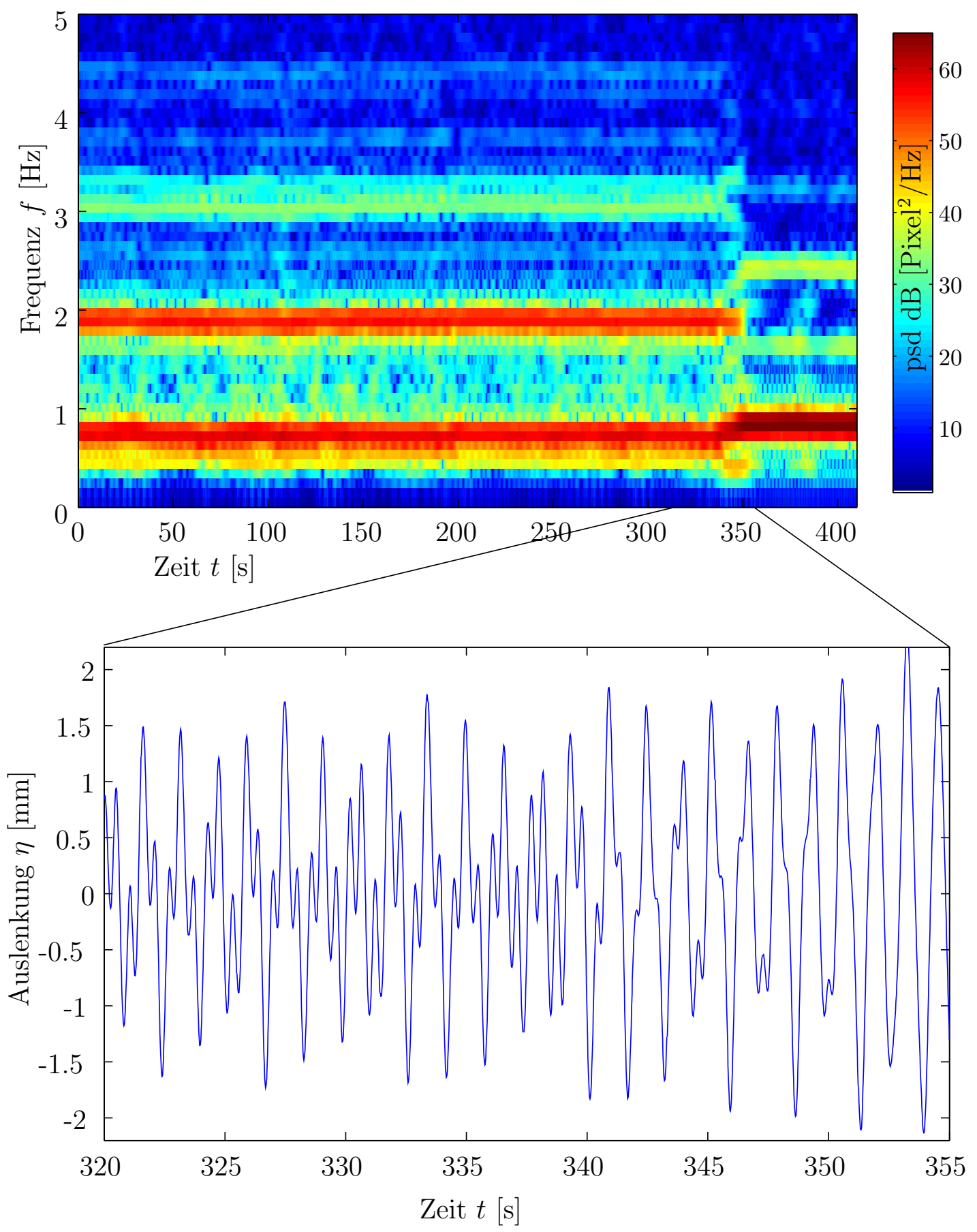

Abb. 4.13: Spontaner Systemwechsel. Übergang des höheren Zustands nach Mode I, L/d $=7, \mathrm{Re}_{\mathrm{d}}=230$. Oben: Spektrogramm, wobei die Farbe die spektrale Leistungsdichte kodiert. Unten: Ausschnitt der Zeitreihe beim Übergang. 


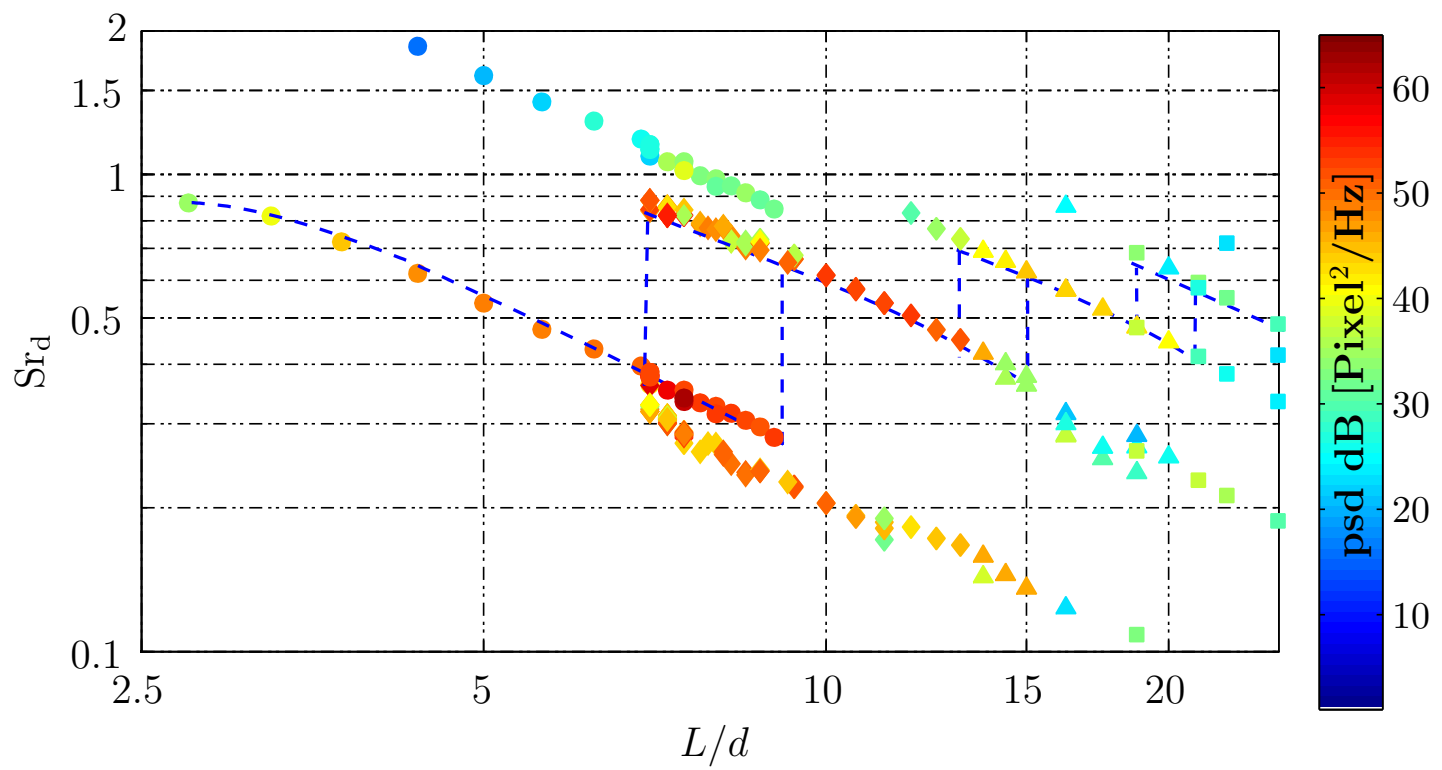

Abb. 4.14: Peakfrequenzen (Strouhalzahlen) in Abhängigkeit vom Keilabstand. ○ Mode I, $\diamond$ Systemzustand II, $\triangle$ Systemzustand III, $\square$ Systemzustand IV.

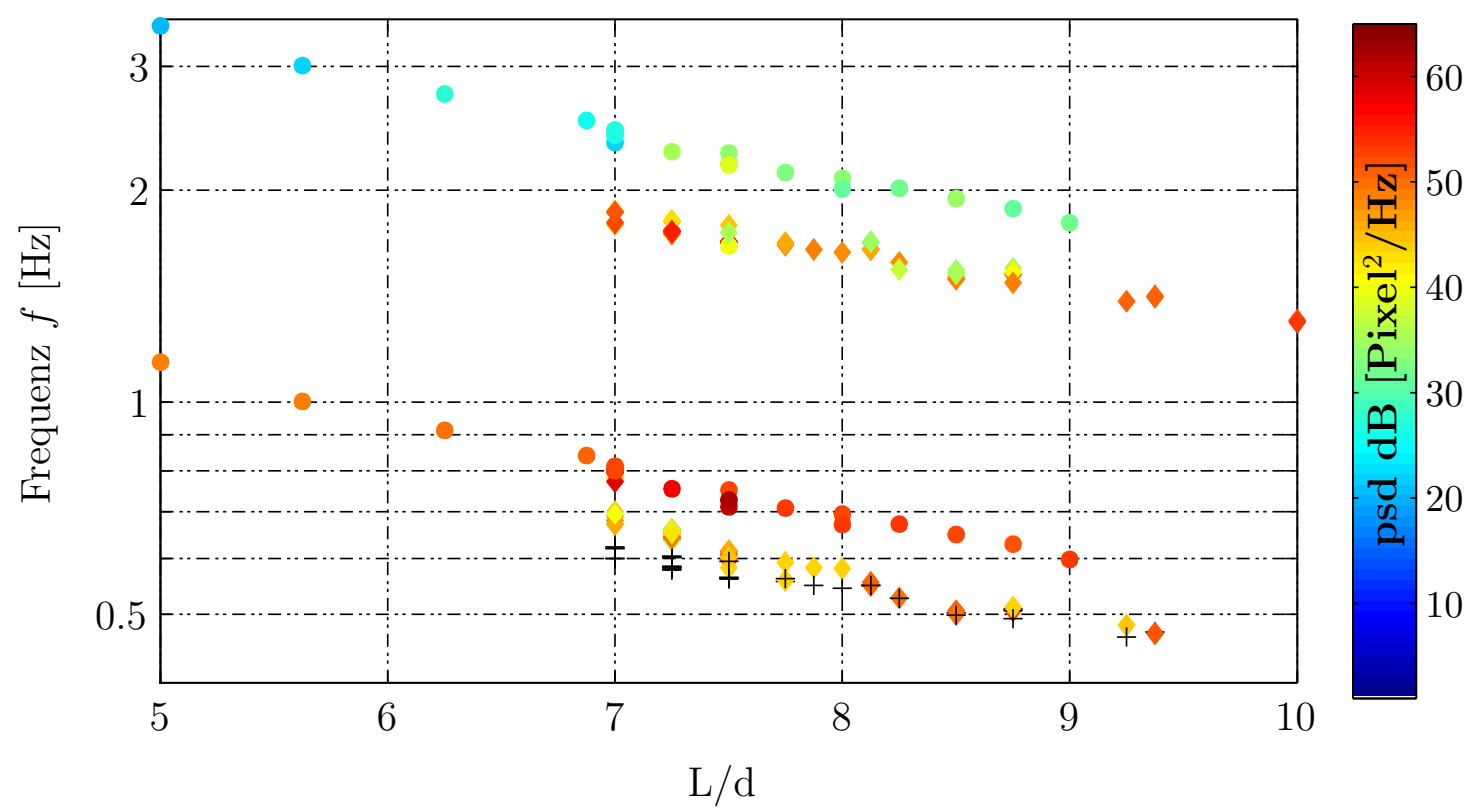

Abb. 4.15: Peakfrequenzen (Strouhalzahlen) in Abhängigkeit vom Keilabstand. ○ Mode I, $\diamond$ Systemzustand II. Die Kreuze kennzeichnen 1/3 der höheren Frequenz von Systemzustand II. 
Kapitel 4. Struktur und Dynamik der Moden

frequenzen des Leistungsspektums sind in Abb. 4.14 als Funktion von $L / d$ dargestellt. Mit zunehmendem $L / d$ wächst die Anzahl der markanten Frequenzen im Leistungsspektrum. Daher wird anhand der räumlichen Struktur des Systemzustands entschieden, welche der Moden den Systemzustand bestimmt. Dadurch wird eine den Systemzustand $m$ charakterisierende Peakfrequenz $\omega_{\mathrm{m}}$ festgelegt und die zu dieser Frequenz gehörende räumliche Struktur als Mode betrachtet, die für die Auswertung der Moden in Abschnitt 4.2 zugrunde gelegt wurde.

Der niederfrequente Peak (Abb. 4.15) der Systemzustände II liegt jeweils unterhalb der Frequenz $f_{I}$ der reinen Mode $\mathrm{I}\left(f^{\mathrm{T}}<f_{I}\right)$. Andererseits ist $f^{\mathrm{T}}>f^{\mathrm{H}} / 3$ (Kreuze in Abb. 4.15). Damit liegt $f^{\mathrm{T}}$ zwischen $f_{I}$ und einer virtuellen Subharmonischen $f^{\mathrm{H}} / 3$. Bei höheren Systemzuständen (Abb. 4.14) sieht man Ähnliches. Die niederfrequenteren Spitzen liegen in der Nähe, der auf den Abstand $L$ extrapolierten charakteristischen Frequenz des nächst niedrigeren Systemzustands. Dabei kann es auch vorkommen, dass das Frequenzverhältnis größer als 3 ist, wenn die Frequenz des nächst niedrigeren Systemzustands kleiner als 1/3 der Frequenz des höherfrequenten Peaks ist. Daher liegt es nahe anzunehmen, dass sich die höheren Systemzustände aus mehreren miteinander gekoppelten Moden zusammensetzen, so dass es zu diesen Frequenzverschiebungen kommt.

\subsection{Dynamik der Moden}

Die höheren Systemzustände beinhalten mehrere Moden, deren Zusammenspiel innerhalb eines Systemzustandes untersucht werden soll. Dabei stellt sich heraus, dass die Moden innerhalb eines Systemzustandes synchronisieren. Die Momentanfrequenzen der einzelnen Moden stehen in einem rationalen Frequenzverhältnis, das nahe bei 3:1 liegt, allerdings mit großen Zählern (11:4, 17:6, 35:12, 47:16). Die Momentanfrequenzen schwanken zeitlich. Jedoch ziehen sich die Frequenzen zueinander hin, so dass die Synchronisation im Mittel erhalten bleibt, oder das System springt in einen anderen Synchronisationszustand. Somit durchwandert das System verschiedene Synchronisationszustände: Zunächst laufen die Moden synchron z. B. mit dem Frequenzverhältnis 1:3, nach mehreren Dekaden Schwingungen rasten die Moden in einem anderen Verhältnis ein, z. B. 17:6. Nach einer gewissen Dauer synchronisieren die Moden wieder bei einem 1:3 Verhältnis und so fort. Die Verweildauer der unterschiedlichen Zustände ist teilweise sehr unterschiedlich. Es ist erstaunlich, dass derart hohe rationale Verhältnisse bei der Synchronisation auftreten. Damit verbunden ist ein entsprechend langes Gedächtnis der Strömung. Es wurden rationale Verhältnisse bis zu 47:16 gefunden. Dies entspricht bei einer Frequenz der höheren Mode von $0.88 \mathrm{~Hz}$ einem Gedächtnis von etwa 53 Sekunden. Untersucht man die räumliche Struktur von Mode I und Mode II im Systemzustand II, so durchdringen sich die Moden relativ ungestört. Die räumliche Struktur hängt für jede Mode nur in etwa von der jeweiligen Frequenz ab. Die Wechselwirkung der Moden findet daher wahrscheinlich durch die Wechselwirkung des Strahls mit dem Keil statt. Dort könnte sich zum Beispiel eine langlebige 
Strömung etablieren, die die einzelnen Moden gemeinsam steuert und so die Synchronisation bewirkt.

\subsubsection{Synchronisation der Moden}

Um eine Synchronisation zwischen den Moden nachzuweisen, müssen zunächst die Zeitreihen der einzelnen Moden des betreffenden Systemzustandes aus der gemeinsamen Zeitreihe $\eta\left(x_{\text {mess }}, t\right)$ der Streichlinienauslenkung am Messort $x_{\text {mess }}$ extrahiert werden. Das Leistungsspektrum von $\eta\left(x_{\text {mess }}, t\right)$ zeigt für den Systemzustand II, der im Folgenden betrachtet wird, zwei Spitzen etwa gleicher Leistungsdichte (siehe auch Abb. 4.15). Der tieffrequente Anteil der Zeitreihe kann zur Charakterisierung von Mode I verwendet werden, der hochfrequente für Mode II. Eine zwischen den Peakfrequenzen des Leistungsspektrums liegende Frequenz wird als Obergrenze des Tiefpasses (TP) und Untergrenze des Hochpasses (HP) gewählt (hier das arithmetische Mittel), so dass das Signal zu

$$
\eta\left(x_{\text {mess }}, t\right)=\eta^{\mathrm{TP}}\left(x_{\text {mess }}, t\right)+\eta^{\mathrm{HP}}\left(x_{\text {mess }}, t\right)
$$

zerlegt wird. Da im Folgenden die Modulationen der Momentanfrequenzen von $\eta^{\mathrm{TP}}$ und $\eta^{\mathrm{HP}}$ untersucht werden, müssen möglichst breite Bandpässe verwendet werden, damit auch die Modulationstransferfunktion möglichst breitbandig ist.

Die Synchronisation äußert sich in einem rationalen Verhältnis der Phasenbeziehung von Mode I zu Mode II:

$$
m \cdot \arg \left(\eta^{\mathrm{HP}}\right)-n \arg \left(\eta^{\mathrm{TP}}\right) \approx \text { const. },
$$

wobei kleine Schwankungen durch verrauschte Messdaten oder durch die Systemdynamik zugelassen werden (schwache Phasensynchronisation [36]).

\section{Analytisches Signal}

Das Problem liegt nun darin, eine geeignete Phase für $\eta^{\mathrm{TP}}$ und $\eta^{\mathrm{HP}}$ zu definieren. Man kann sich zum Beispiel die Nulldurchgänge der Signale ansehen und zwischen diesen die Phase linear interpolieren; dies ist allerdings nur bei streng mittelwert- und rauschfreien Signalen möglich. Hier wird das analytische Signal benutzt, um die Phase zu definieren. Das analytische Signal $\eta_{\mathcal{C}}^{\mathrm{TP}}$ kann für $\eta^{\mathrm{TP}}$ durch

$$
\eta_{\mathcal{C}}^{\mathrm{TP}}=\eta^{\mathrm{TP}}+i \mathcal{H}\left\{\eta^{\mathrm{TP}}\right\}
$$

und für $\eta^{\mathrm{HP}}$ in gleicher Weise berechnet werden, wobei $\mathcal{H}\left\{\eta^{\mathrm{TP}}\right\}$ die Hilberttransformierte von $\eta^{\mathrm{TP}}$

$$
\mathcal{H}\left\{\eta^{\mathrm{TP}}\right\}=\eta^{\mathrm{TP}} * \frac{1}{\pi t}
$$


ist. Dies kann man auch mittels Fouriertransformation und Faltungssatz ausdrücken als

$$
\mathcal{H}\left\{\eta^{\mathrm{TP}}\right\}=-\mathcal{F}^{-1}\left\{\mathcal{F}\left\{\eta^{\mathrm{TP}}\right\} \cdot i \cdot \operatorname{sign}(\omega)\right\} .
$$

Das Spektrum von $\eta_{\mathcal{C}}^{\mathrm{TP}}$ enthält also nur positive Frequenzen.

Aus der stetig interpolierten - über das Intervall $[0,2 \pi]$ stetig fortgesetzten - Phase $\arg \left(\eta_{\mathcal{C}}^{\mathrm{TP}}\right)$ bzw. $\arg \left(\eta_{\mathcal{C}}^{\mathrm{HP}}\right)$ können die Momentanfrequenzen der Mode I $\frac{d \arg \left(\eta_{\mathcal{C}}^{\mathrm{TP}}\right)}{d t}$ und der Mode II $\frac{d \arg \left(\eta_{\eta_{\mathrm{HP}}}^{\mathrm{HP}}\right)}{d t}$ berechnet werden, die allerdings bei den gemessenen Streichlinienauslenkungen durch die leicht verrauschte Phase erheblich schwanken.

Zum Nachweis der Phasensynchronisation zwischen $\eta^{\mathrm{TP}}$ und $\eta^{\mathrm{HP}}$ kann die Amplitudenverteilung bzw. die Häufigkeitsverteilung (im Folgenden mit PDF abgekürzt (probability density function)) einer geeignet definierten Phasendifferenz $\Psi\left(v^{\mathrm{H} / \mathrm{T}}, t\right)$ untersucht werden:

$$
\Psi\left(v^{\mathrm{H} / \mathrm{T}}, t\right)=\arg \left(\eta_{\mathcal{C}}^{\mathrm{HP}}\right)-v^{\mathrm{H} / \mathrm{T}} \cdot \arg \left(\eta_{\mathcal{C}}^{\mathrm{TP}}\right) .
$$

Ist die Phase in einem bestimmten Zeitintervall $a<t_{1}<b$ bei einem bestimmten $v_{1}^{\mathrm{H} / \mathrm{T}}$ synchronisiert, so ist dort $\Psi\left(v^{\mathrm{H} / \mathrm{T}}, t_{1}\right)$ näherungsweise konstant und die Amplitudenverteilung von $\Psi\left(v_{1}^{\mathrm{H} / \mathrm{T}}, t_{1}\right)$ zeigt eine ausgeprägte Spitze bei $\left\langle\Psi\left(v_{1}^{\mathrm{H} / \mathrm{T}}, t_{1}\right)\right\rangle_{\mathrm{t}_{1}}$. Synchronisieren die Moden in einem anderem Zeitintervall $c<t_{2}<d$ mit $v_{2}^{\mathrm{H} / \mathrm{T}}$, so ist $\left\langle\Psi\left(v_{2}^{\mathrm{H} / \mathrm{T}}, t_{2}\right)\right\rangle_{\mathrm{t}_{2}}$ nicht zwangsläufig $\left\langle\Psi\left(v_{1}^{\mathrm{H} / \mathrm{T}}, t_{1}\right)\right\rangle_{\mathrm{t}_{1}}$ - die Synchronisation rastet nicht unbedingt auf derselben relativen Phasenlage zwischen den Moden ein. Es ergibt sich allerdings wiederum eine Spitze in der Amplitudenverteilung.

In Abb. 4.16 ist der Modenübergang anhand der Zeitreihe (oben) und anhand der Einhüllenden (Mitte), also dem Betrag des analytischen Signals, dargestellt. Nach etwa 260 Schwingungen der höheren Mode mit der Frequenz $f^{\mathrm{H}}=1.76 \mathrm{~Hz}$ geht der Systemzustand II in Mode I über. Für $t \cdot f^{\mathrm{H}}<260$ sind die Amplituden von $\eta^{\mathrm{HP}}$ und von $\eta^{\mathrm{TP}}$ ähnlich, was den Systemzustand II auszeichnet, während für Mode I $\left|\eta_{\mathcal{C}}^{\mathrm{TP}}\right|>\left|\eta_{\mathcal{C}}^{\mathrm{HP}}\right|$ ist. Die Beträge $\left|\eta_{\mathcal{C}}^{\mathrm{TP}}\right|$ und $\left|\eta_{\mathcal{C}}^{\mathrm{HP}}\right|$ sind nicht konstant, sondern fluktuieren mit einer Frequenz, die deutlich kleiner ist als die Frequenz der Mode I. Die langsamen Fluktuationen deuten auf eine Wechselwirkung hin, die zum Modenübergang hin stärker wird.

Trägt man für das Frequenzverhältnis $v^{\mathrm{H} / \mathrm{T}}=3$ die Differenzphase $\Psi(3, t)=\arg \left(\eta_{\mathcal{C}}^{\mathrm{HP}}\right)-$ $3 \arg \left(\eta_{\mathcal{C}}^{\mathrm{TP}}\right)$ auf, so ist z.B. $\Psi\left(3, t_{1}\right)$ für $55<t_{1} \cdot f^{\mathrm{H}}<110$ und $\Psi\left(3, t_{2}\right)$ für $135<t_{2} \cdot f^{\mathrm{H}}<175$ etwa konstant. Man sieht auch zwischen diesen Hauptplateaus, dass die Phase stets bei ganzzahligen Vielfachen von $2 \pi$ einrastet. Für $v^{\mathrm{H} / \mathrm{T}}=2.77$ gibt es zwar auch solche Hauptplateaus $\left(110<t_{1} \cdot f^{\mathrm{H}}<135\right.$ oder $\left.200<t_{2} \cdot f^{\mathrm{H}}<270\right)$, aber $\Psi\left(2.77, t_{1 / 2}\right)$ fluktuiert stärker, und es gibt für dieses Frequenzverhältnis keine bevorzugte Phasendifferenz, bei der das System einrastet.

Eine Synchronisation erzeugt also zunächst eine Ungleichverteilung der Phasenhäufigkeit von $\Psi\left(v^{\mathrm{H} / \mathrm{T}}, t\right)$. Als Maß für die Ungleichverteilung kann die Varianz der Verteilung, also die mittlere quadratische Abweichung der Häufigkeitsverteilung $\operatorname{PDF}\left(\Psi\left(v^{\mathrm{H} / \mathrm{T}}, t\right)\right)$ von der Gleichverteilung über dem entsprechenden $\Psi$-Intervall benutzt werden. 
Normiert man $\Psi\left(v^{\mathrm{H} / \mathrm{T}}, t\right)$ auf das Intervall $[0,1]$ durch Normierung mit

$$
\Psi^{0}\left(v^{\mathrm{H} / \mathrm{T}}, t\right)=\frac{\Psi\left(v^{\mathrm{H} / \mathrm{T}}, t\right)}{\Delta \Psi} \text { mit } \Delta \Psi=\max _{t}\left(\Psi\left(v^{\mathrm{H} / \mathrm{T}}, t\right)\right)-\min _{t}\left(\Psi\left(v^{\mathrm{H} / \mathrm{T}}, t\right)\right),
$$

so ist die Häufigkeitsverteilung der Phase durch

$$
P D F\left\{\Psi^{0}\left(v^{\mathrm{H} / \mathrm{T}}, t\right)\right\}=P D F\left\{\Psi\left(v^{\mathrm{H} / \mathrm{T}}, t\right)\right\} \cdot \Delta \Psi
$$

gegeben. Die mittlere quadratische Abweichung der $P D F\left\{\Psi^{0}\left(v^{\mathrm{H} / \mathrm{T}}, t\right)\right\}$ von der Gleichverteilung $P D F \equiv 1$ ist somit

$$
\left\langle\left[1-P D F\left\{\Psi^{0}\left(v^{\mathrm{H} / \mathrm{T}}, t\right)\right\}\right]^{2}\right\rangle_{\Psi}=\left\langle P D F\left\{\Psi^{0}\left(v^{\mathrm{H} / \mathrm{T}}, t\right)\right\}^{2}\right\rangle_{\Psi}-1 .
$$

In Abb. 4.17 ist die Varianz der Phasenverteilung für die gesamte Differenzphase $\Psi$ mit der Differenzphase $\Psi$ mod $2 \pi$ verglichen. Betrachtet man $\Psi$ nur auf dem Intervall $[0,2 \pi]$, so mitteln sich in einer langen Zeitreihe, in der sich die Synchronisation ständig ändert, die Anteile, für $\operatorname{die}\left\langle\Psi\left(v_{\mathrm{k}}^{\mathrm{H} / \mathrm{T}}, t_{1}\right)\right\rangle_{\mathrm{t}_{1}} \neq\left\langle\Psi\left(v_{\mathrm{k}}^{\mathrm{H} / \mathrm{T}}, t_{2}\right)\right\rangle_{\mathrm{t}_{2}} \neq \cdots \neq\left\langle\Psi\left(v_{\mathrm{k}}^{\mathrm{H} / \mathrm{T}}, t_{\mathrm{n}}\right)\right\rangle_{\mathrm{t}_{\mathrm{n}}}$ gilt, heraus und es bleiben nur Synchronisationen $v_{r m k}^{\mathrm{H} / \mathrm{T}}$ mit gleicher relativer Phasenlage übrig (siehe Abb. 4.17 oben). Für $v^{\mathrm{H} / \mathrm{T}}=3$ (braune Linie) ergibt sich eine Phasensynchronisation und für $v^{\mathrm{H} / \mathrm{T}}=2.825$ (grüne Linie) eine geringe Abweichung von der Gleichverteilung. Die orange und blaue Kurve zeigen noch weniger Varianz. Dies ist in Abb. 4.17 (unten) für alle Frequenzverhältnisse in der grünen Kurve zusammengefasst. Bei $v^{\mathrm{H} / \mathrm{T}}=3$ ist die Varianz am größten und zeigt dort eine scharfe Spitze. Die kleine Spitze bei $v^{\mathrm{H} / \mathrm{T}}=2.825=113 / 40$ entspricht dem Mittel von 17/6 und 31/11. Die oben erwähnten Fluktuationen von $\Psi\left(2.77, t_{1 / 2}\right)=\arg \left(\eta_{\mathcal{C}}^{\mathrm{HP}}\right)-2.77 \cdot \arg \left(\eta_{\mathcal{C}}^{\mathrm{TP}}\right)$ finden sich direkt in den Fluktuationen $\left|\eta_{\mathcal{C}}^{\mathrm{TP}}\right|$ wieder. In Abb. 4.16 (Mitte) ist das Schwankungsmuster für die verschiedenen Synchronisationszustände deutlich verschieden und für $v^{\mathrm{H} / \mathrm{T}}=2.77$ sind die Schwankungen deutlich größer als im relativ glatten Synchronisationszustand mit $v^{\mathrm{H} / \mathrm{T}}=3$. Dieser Aspekt wird in Abschnitt 4.7.2 ausführlich diskutiert.

Bisher wurde das analytische Signal einer Zeitreihe betrachtet. Um die komplette Information aller Zeitreihen auszunutzen, kann eine in Anhang F.1 beschriebene spezielle Karhunen-LoèveTransformation vorgenommen werden, welche analytische Projektionskoeffizienten $p_{\mathcal{C}}$ liefert. In Abb. 4.16 sind die Projektionskoeffizienten $p_{\mathcal{C}}$ und die auf die $p_{\mathcal{C}}$ skalierten analytischen Signale $\eta_{\mathcal{C}}$ (Messort $x_{\text {mess }}=x_{\mathrm{D}}+L / 2$ ) verglichen. Die Schwankungen der Beträge der Projektionskoeffizienten sind etwas kleiner, die Phasenschwankungen sind in ähnlicher Größenordnung (hier anders skaliert). Im Folgenden werden die Projektionskoeffizienten zur Auswertung benutzt, da sie das gesamte Feld repräsentieren.

\subsubsection{Tieffrequente Dynamik der Moden}

Die Dynamik der Moden innerhalb eines Systemzustands findet auf drei verschiedenen Zeitskalen statt: 
Kapitel 4. Struktur und Dynamik der Moden
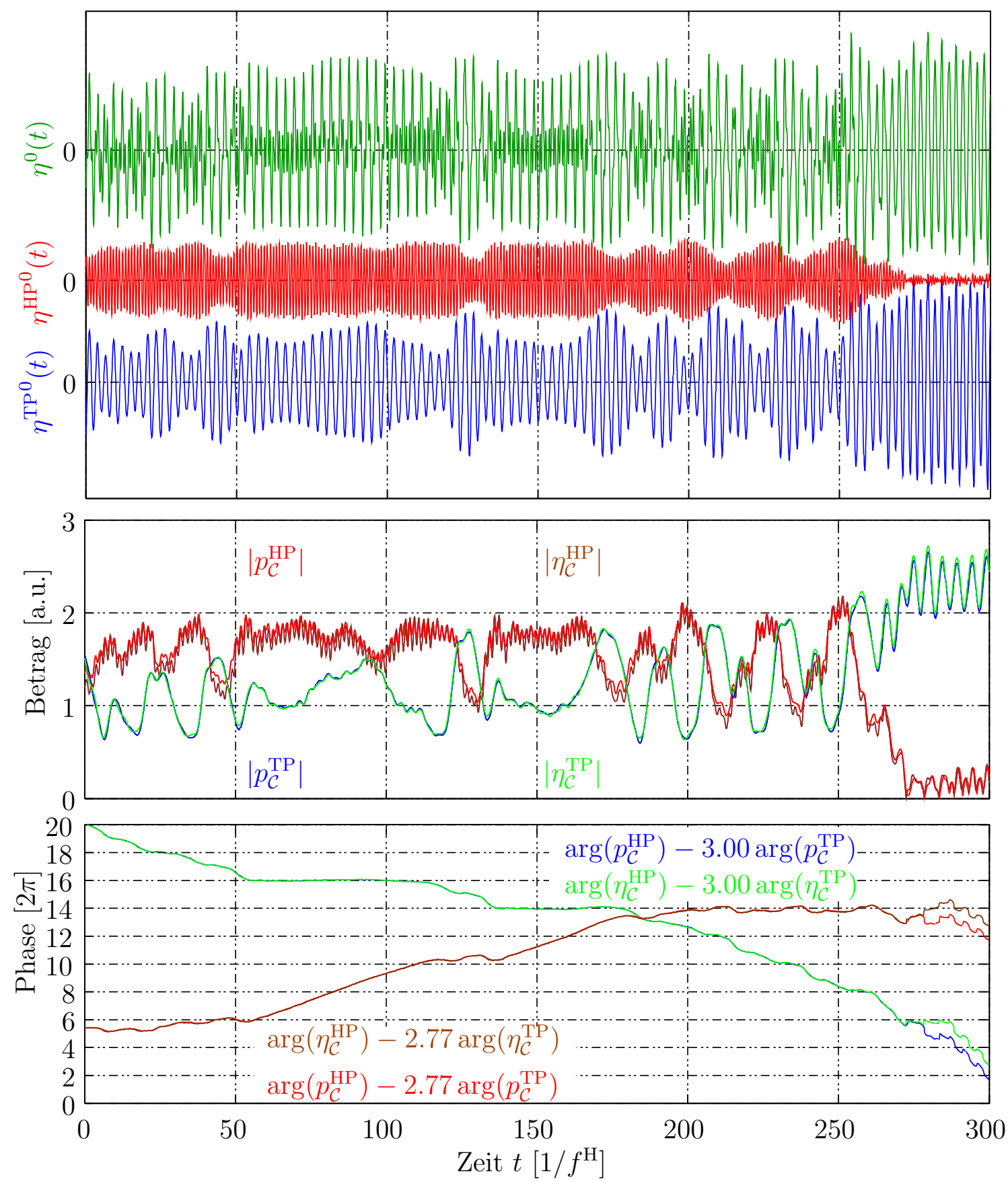

Abb. 4.16: Natürlicher Modenübergang von Mode II zu Mode I ohne Steuersignal. Oben: Zeitreihen der gesamten und nach Mode I und Mode II aufgeteilten, selbsterregten Schwingung. Mitte und unten: Betrag und Phase der Projektionskoeffizienten bzw. des analytischen Signals. Die Differenzphasen sind aus Darstellungsgründen verschoben worden und beginnen beide bei $-0.28 \cdot 2 \pi$. Parameter: $\mathfrak{R e}=230, d=4 \mathrm{~mm}, U_{\mathrm{D}}=5.36 \mathrm{~cm} / \mathrm{s}, L / d=7.25, f^{\mathrm{H}}=1.76 \mathrm{~Hz}$. 

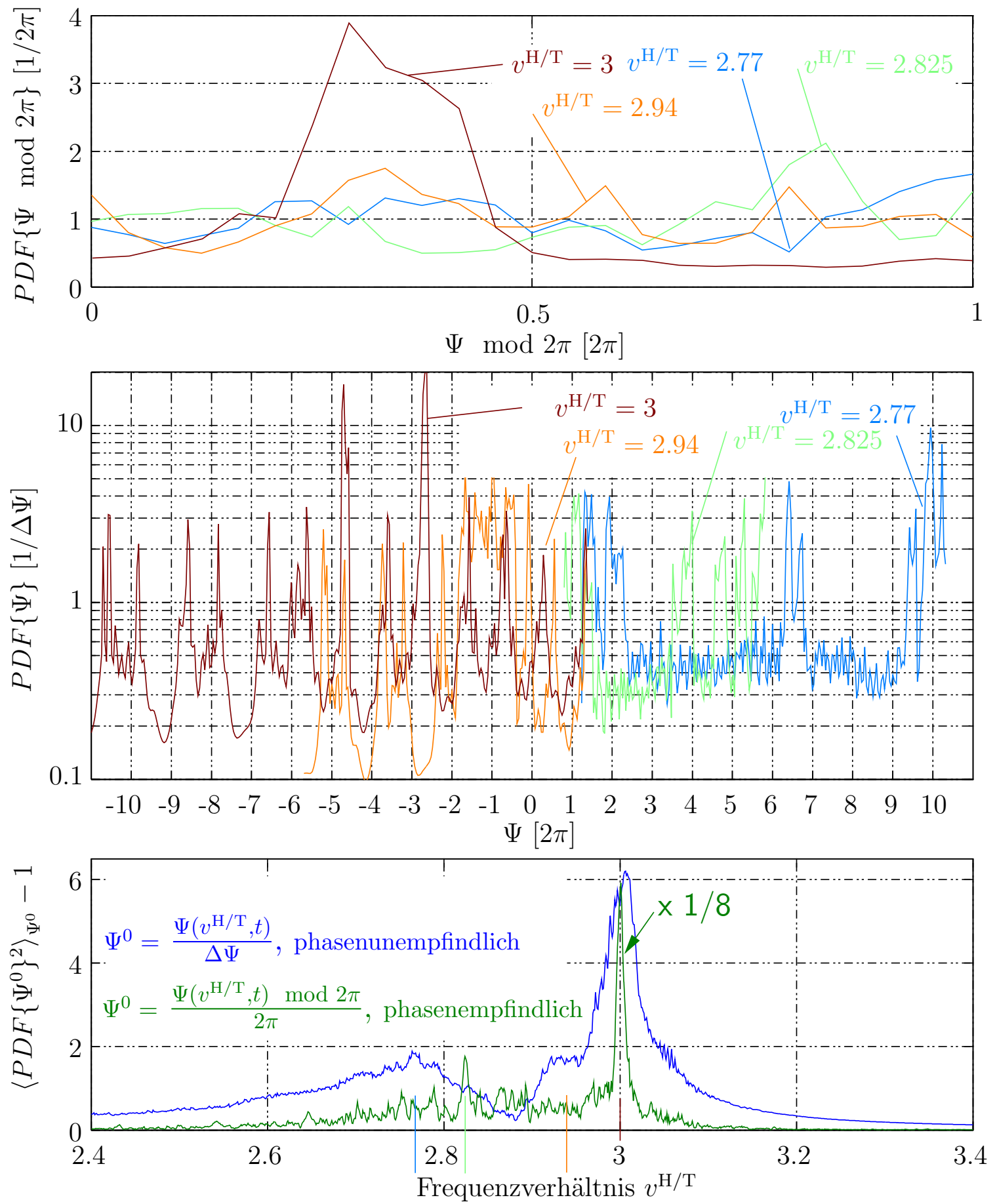

Abb. 4.17: Natürlicher Modenübergang von Mode II zu Mode I ohne Steuersignal. Oben: Phasenverteilung mod $2 \pi$ für verschiedene Frequenzverhältnisse. Mitte: Verteilung der kontinuisierten Phase für verschiedene Frequenzverhältnisse. Unten: Varianz der Phasenverteilung. Parameter: $\mathfrak{R e}=230, d=4 \mathrm{~mm}, U_{\mathrm{D}}=5.36 \mathrm{~cm} / \mathrm{s}, L / d=7.25$. 
- Die einzelnen Moden schwingen mit großer Amplitude auf ihrer jeweiligen Frequenz $f^{\mathrm{T}}$ und $f^{\mathrm{H}}$.

- Aufgrund der Modensynchronisation besteht (für bestimmte Zeitintervalle) ein rationales Frequenzverhältnis $v^{\mathrm{H} / \mathrm{T}}=f^{\mathrm{H}} / f^{\mathrm{T}} \approx m / n$. Die Differenzphase $\Psi(m / n, t)$ schwankt typischerweise mit der Frequenz $f^{\mathrm{H}} / m$ bzw. $f^{\mathrm{T}} / n$.

- Die Phasen der Moden lassen sich in einen linear mit der Zeit anwachsenden Anteil und einen schwankenden Rest

$$
\Delta \Phi^{\mathrm{TP}}=\arg \left(p_{\mathcal{C}}^{\mathrm{TP}}\right)-\left\langle\frac{d \arg \left(p_{\mathcal{C}}^{\mathrm{TP}}\right)}{d t}\right\rangle_{\mathrm{t}} \cdot t=\arg \left(p_{\mathcal{C}}^{\mathrm{TP}}\right)-f^{\mathrm{T}} t
$$

zerlegen. Für den hochfrequenten Projektionskoeffizienten $p_{\mathcal{C}}^{\mathrm{HP}}$ ist das ganz analog möglich. Um die Phasenänderungen auf der Zeitskala von $f^{\mathrm{T}} \approx f^{\mathrm{H}} / 3$ aus dieser alle Zeitskalen enthaltenden Darstellung zu eliminieren, kann man $\Delta \Phi^{\mathrm{TP}}$ noch einmal einer Tiefpassfilterung mit $f^{\mathrm{H}} / 4$ als oberer Grenzfrequenz unterziehen, was durch ein weiteres $T P$ an $\Delta \Phi^{\mathrm{TP}}-$ also $\Delta \Phi^{\mathrm{TP} \mathrm{TP}}$ - gekennzeichnet sein soll. Die tieffrequenten Schwankungen von $p_{\mathcal{C}}^{\mathrm{HP}}$ und $p_{\mathcal{C}}^{\mathrm{TP}}$ enthalten zum einen die im letzten Punkt erwähnte Synchronisationsfrequenz $f^{\mathrm{H}} / m=f^{\mathrm{T}} / n$ und zusätzlich eine noch wesentlich tieffrequentere Dynamik, wobei $\Delta \Phi^{\mathrm{TP} T P}$ und $\Delta \Phi^{\mathrm{HP}}$ stets in ähnlicher Phasenlage auftreten. Dies lässt auf eine Kopplung der Moden in dieser tieffrequenten Dynamik schließen. Überschreitet die Differenz zwischen $\Delta \Phi^{\mathrm{TP}}$ und $\Delta \Phi^{\mathrm{HP}}$ eine gewisse Schwelle, so wechselt entweder das Synchronisationsverhältnis innerhalb eines Systemzustandes oder das System geht sogar in einen anderen Zustand über, beides ist in Abb. $4.16 \mathrm{zu}$ sehen.

Um zunächst einen Eindruck von den verschiedenen Zeitskalen zu vermitteln, ist in Abb. 4.18 eine Videosequenz des Systemzustands II mit dem Synchronisationsverhältnis 8:3 gezeigt. Dabei wurde die Farbstoffinjektion so nach rechts verschoben, dass die rechte Scherschicht sichtbar wird. Das linke Gebiet und der Strahl sind etwas heller, da der Farbstoff hauptsächlich auf der rechten Kanalseite bleibt. Die Zeitskala, die in jeder Zeile der Abb. 4.18 eingezeichnet ist, ist normiert auf die Schwingungsdauer $1 / f^{\mathrm{H}}=1 / 1.847 \mathrm{~Hz}$ der Mode II. Jedem Bild ist durch eine rote Markierung auf der Zeitachse seine Phasenlage zugeordnet. Da die Samplingfrequenz $f_{\text {sampling }}=6.25 \mathrm{~Hz} \neq j f^{\mathrm{H}}, j \in \mathcal{N}$ ist, verschiebt sich die Phasenlage der Einzelbilder von Schwingung zu Schwingung. Allerdings ist bei dieser ausgewählten Messung $9 \cdot 3 / f_{\text {sampling }} \approx$ $8 / f^{\mathrm{H}}$, so dass die Bilder der Zeile $\mathrm{D}$ in Abb. 4.18 bezogen auf $f^{\mathrm{H}}$ dieselbe Phasenlage haben, also äquivalent zu der Zeile A sind.

Die Ausbreitung der Instabilitätswelle kann leicht verfolgt werden, weil sich am Strahlrand die Scherschicht aufrollt und den Farbstoff ansammelt. Die Ausbreitung dieser Ansammlungen ist in Abb. 4.18 durch Linien markiert. Die Scherschichtauslenkung breitet sich mit unterschiedlicher Geschwindigkeit stromab aus, wobei drei wesentlich verschiedene Ausbreitungsgeschwin- 

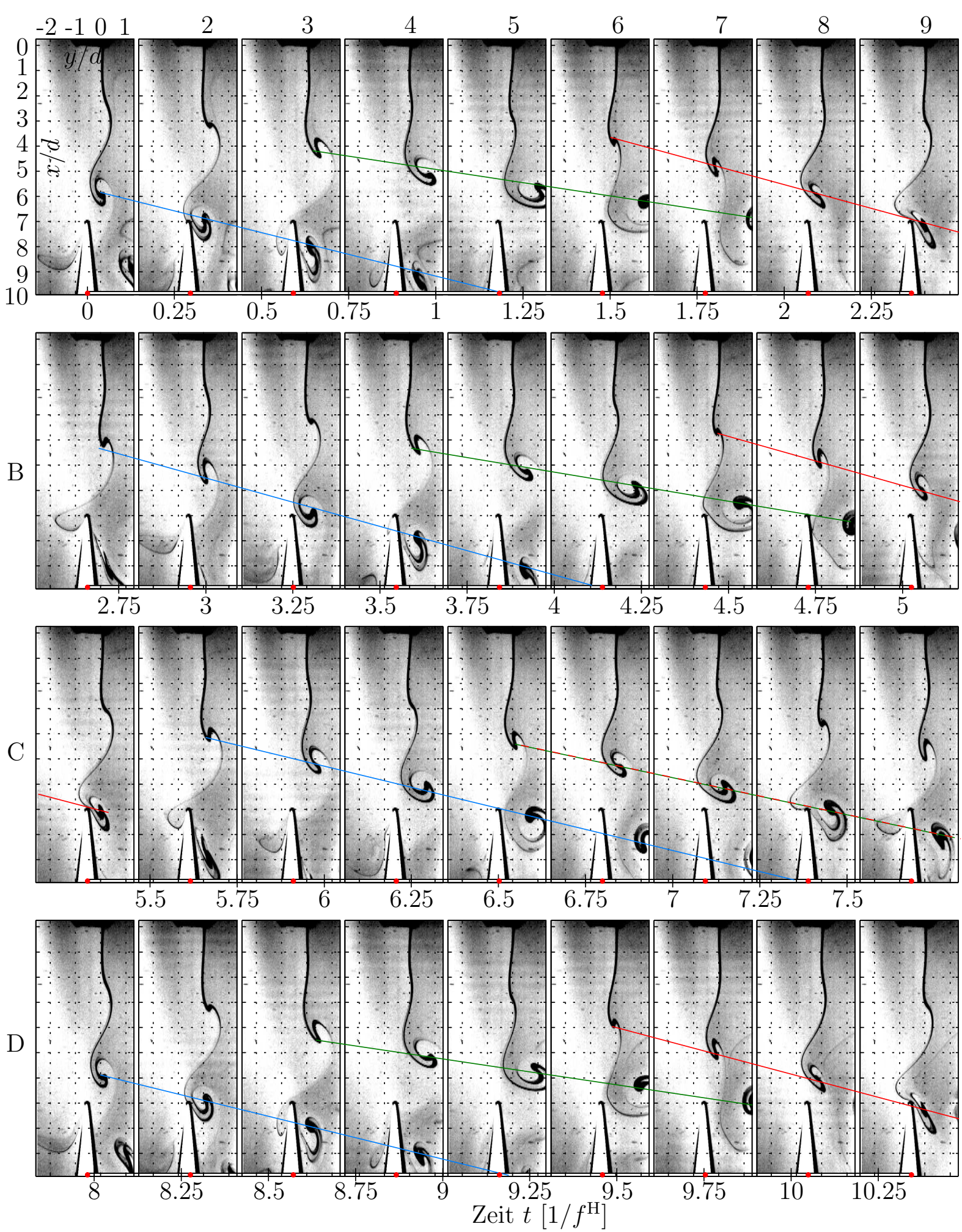

Abb. 4.18: Videobildersequenz einer 8:3 Synchronisation. rechte Scherschicht ist eingefärbt. Gitterabstand $=4 \mathrm{~mm}=d$. Parameter: $L / d=7, U_{\mathrm{D}}=5.36 \mathrm{~cm} / \mathrm{s}, f^{\mathrm{H}}=1.85 \mathrm{~Hz}$. 
Kapitel 4. Struktur und Dynamik der Moden

digkeiten bzw. Muster auftreten, denen jeweils eine bestimmte Farbe zugeordnet wurde. Die Ausbreitungsgeschwindigkeit nimmt zu, je kleiner die Scherschichtauslenkung ist.

Die grüne Linie kennzeichnet die Schwingung mit der größten Auslenkung, was eine geringere Ausbreitungsgeschwindigkeit der Wirbel in der Scherschicht zur Folge hat. Der Wirbel passiert die horizontale Keilebene $x / d=L / d=7$ weit rechts (Bildrand von Bild A7) bei etwa $y=1 d$. Die Ausbreitungsgeschwindigkeit der Scherschicht ist bei den Schwingungen, die mit rot und blau markiert sind, etwa gleich. Die Positionen und Ausbreitungsmuster der Wirbel unterscheiden sich insbesondere beim Passieren der Keilebene. Die Farbstoffansammlungen haben nach dem Auftreffen des Strahls auf den Keil unterschiedliche Geschwindigkeiten, in Bild A2 und A3 breitet sich der Wirbel mit gleichbleibender Geschwindigkeit aus (blaue Linie), während sich die Geschwindigkeit des Wirbels in Bild C1 und C2 stark vergrößert (rote Linie). In den dazu äquivalenten Bilderpaaren findet sich ähnliches.

Bei einem Synchronisationsverhältnis von 3:1 müsste sich das Schwingungsmuster, das hier anhand der über der Zeit kontinuierlichen Ausbreitung charakterisiert wird, exakt nach $3 / f^{\mathrm{H}}$ wiederholen. Betrachtet man dazu die Ausbreitung der weit nach rechts ausgelenkten Scherschicht (Schema grün), so ist in Zeile A, Zeile B und Zeile D das Ausbreitungsmuster sehr ähnlich - der rechte Strahlrand geht beim Passieren der Keilebene genau rechts vom Keil vorbei (A6, B7, D6) - nur in Zeile C wird die Scherschicht durchschnitten (C8-C9). Damit wird die 1:3 Periodizität gestört. In Zeile C findet man anstelle der zwei Schwingungsperioden (der Schwingung nach Schema rot und der nach Schema grün) nur eine Schwingungsperiode, die dem „gemittelten“ Ausbreitungsmuster dieser beiden Schwingungsperioden entspricht. Danach wiederholt sich in Zeile D sehr exakt wieder die Zeile A. Da die Ausbreitungsmuster in Zeile A und Zeile B sehr ähnlich sind und sich dann stark in Zeile C unterscheiden, scheint der Vorgang sich zunächst allmählich aufzubauen, um dann sprunghaft wieder von vorne zu beginnen.

Dies erkennt man auch an der Ausbreitung nach dem Schema blau. Die Steigungen sind in allen Zeilen etwa gleich, allerdings ist der Schnittpunkt der blauen Linien mit einer gedachten Linie $x / d=10$ in Zeile A und Zeile B zu Modulo $1 / f^{\mathrm{H}}$ fast äquivalenten Zeitpunkten $\left(1.23 / f^{\mathrm{H}}\right.$ und $\left.4.20 / f^{\mathrm{H}}\right)$ und unterschiedet sich stark in Zeile $\mathrm{C}\left(7.40 / f^{\mathrm{H}}\right)$.

Um dieses Symptom der Synchronisation noch genauer darzustellen, wurde jedem Videobild in Abb. 4.19 am rechten Rand eine Spalte (rote Linie in Abb. 4.19) entnommen und in zeitlicher Reihenfolge aneinandergesetzt. Damit wird die $x$-Position des Wirbels auf der Scherschicht sichtbar, bei der die rote Linie passiert wird. Auf diese Weise wird nur die Struktur der am weitesten nach rechts ausgelenkten Schwingung (Schema grün) aus Abb. 4.18 sichtbar, da die Verwirbelungen nur in dieser Phase der Synchronistationsperiode bis zum Bildrand vorstoBen. Das in Abb. 4.19 periodisch mit der Wiederholfrequenz von $f^{\mathrm{H}} / 8$ wiederkehrende Muster zeigt zum einen die Synchronisationsperiode und zum anderen zeigen die Schwankungen dieses Musters die tieffrequente Dynamik oder die stochastischen Schwankungen des Systems. 


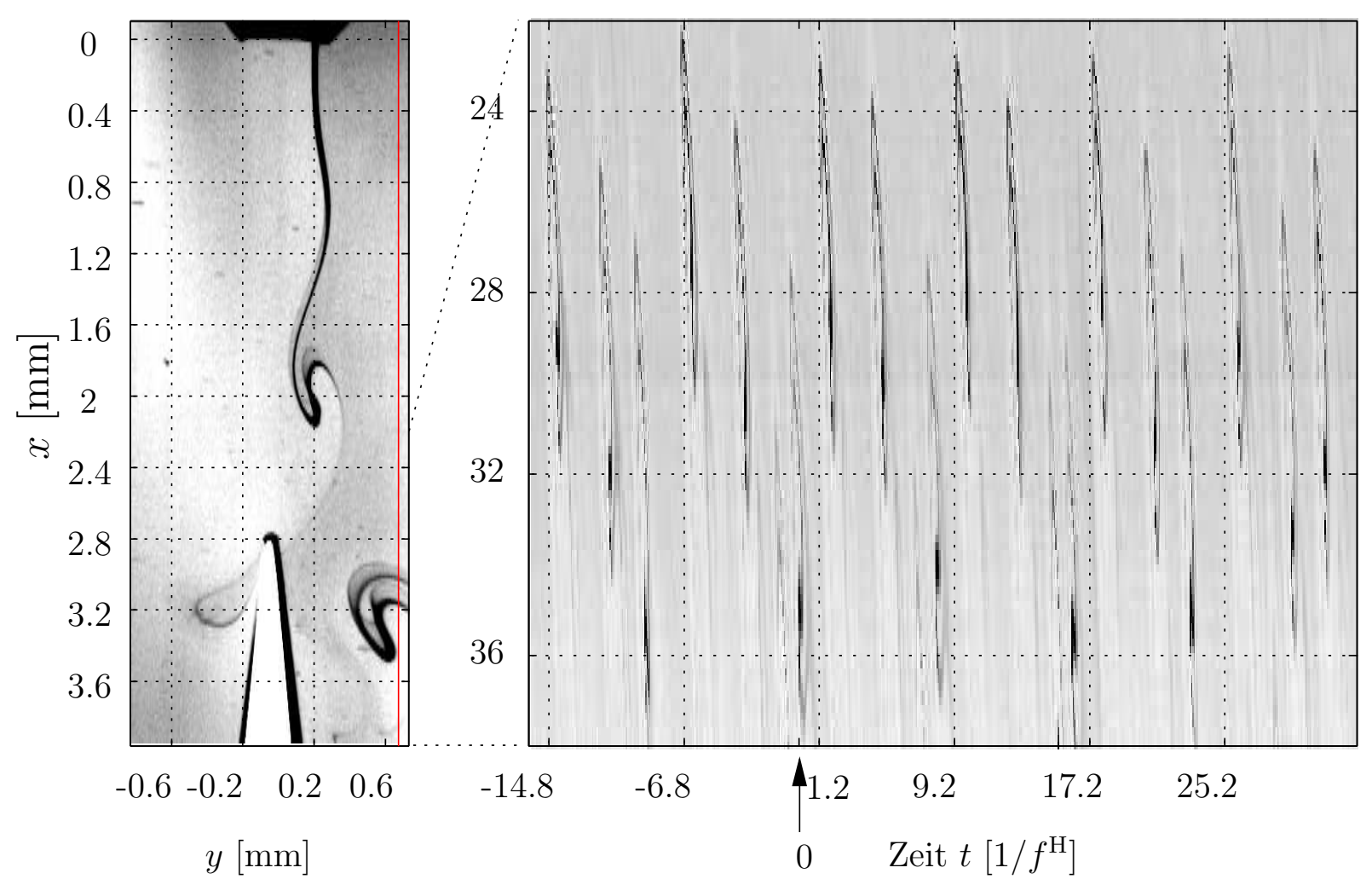

Abb. 4.19: 8:3 Synchronisation der Moden. Die auf der rechten Seite dargestellte Streakline wurde am rechten Rand (rote Linie) der Videobilder entnommen. Bei der Messung wurde die rechte Scherschicht eingefärbt. Parameter: $L / d=7, U_{\mathrm{D}}=5.36 \mathrm{~cm} / \mathrm{s}, d=4 \mathrm{~mm}, f^{\mathrm{H}}=1.85 \mathrm{~Hz}$.

In den Abbildungen 4.20, 4.22 und 4.23 sind andere Synchronisationsverhältnisse und verschiedene Hauptfrequenzverhältnisse zwischen hoher und tiefer Mode in Systemzustand II dargestellt. Die Frequenzverhältnisse sind nicht stationär, was anhand der Differenzphase $\Psi$ untersucht wird.

Die Differenzphase schwankt typischerweise mit der Periodenlänge der Synchronisation $n / f^{\mathrm{H}}$ bzw. $m f^{\mathrm{T}}$, wobei $n, m$ durch das Frequenzverhältnis $v^{\mathrm{H} / \mathrm{T}}=n / m$ der Synchronisation gegeben sind. In Abb. 4.20 (unten) werden relativ konstant 11 Schwingungen der Mode II ausgeführt, während die Mode I nur 4 mal schwingt. Die Differenzphase $\Psi(11 / 4, t)$ zeigt genau diese Periodizität. Dabei wird $\Psi$ durch einen Tiefpass mit der oberen Grenzfrequenz $f^{\mathrm{H}} / 4$ gefiltert, um die höherfrequenten Schwankungen mit den Frequenzen $f^{\mathrm{T}}$ und $f^{\mathrm{H}}$ zu beseitigen und $\Psi$ geht damit in $\Psi^{\mathrm{TP}}$ über. Dadurch lässt sich in Abb. 4.20 (unten) eine tieffrequente Schwingung erkennen, deren Periodendauer $11 / f^{\mathrm{H}}$, bzw. $4 / f^{\mathrm{T}}$ ist.

Die Schwankung der Phasen $\arg \left(p_{\mathcal{C}}^{\mathrm{HP}}\right)$ und $\arg \left(p_{\mathcal{C}}^{\mathrm{TP}}\right)$ um die mittlere Steigung (also $\Delta \Phi^{\mathrm{TP}}{ }^{\mathrm{TP}}$ und $\Delta \Phi^{\mathrm{HP}}$ ) in Abb. 4.21 lassen erkennen, dass $\Delta \Phi^{\mathrm{TP} T P}$ und $\Delta \Phi^{\mathrm{HP}}{ }^{\mathrm{TP}}$ nicht unabhängig voneinander sind. Die Phasen scheinen sich gegenseitig anzuziehen (z.B. $\left.t \cdot f^{\mathrm{H}}>1000\right)$. Betrachtet 

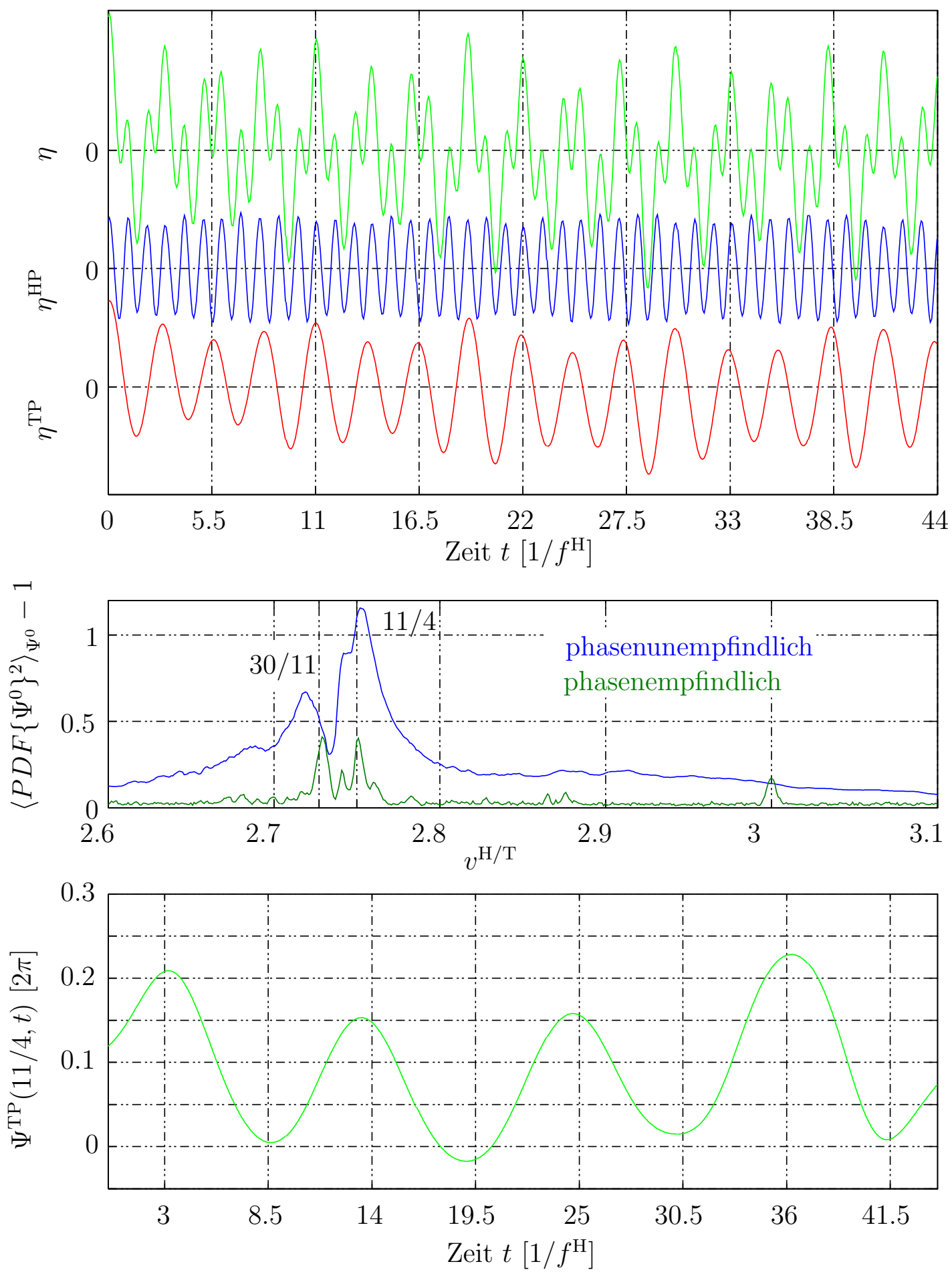

Abb. 4.20: Hauptfrequenzverhältnis 11/4. Oben: Zeitreihe. Mitte: Varianz der Phasenverteilung. Unten: $\left(\arg \left(p_{\mathcal{C}}^{\mathrm{HP}}\right)-11 / 4 \cdot \arg \left(p_{\mathcal{C}}^{\mathrm{TP}}\right)\right)^{\mathrm{TP}}$. Parameter: $L / d=7.25, U_{\mathrm{D}}=5.36 \mathrm{~cm} / \mathrm{s}$, $d=4 \mathrm{~mm}, f^{\mathrm{H}}=1.75 \mathrm{~Hz}, \mathrm{Re}_{\mathrm{d}}=250$. 


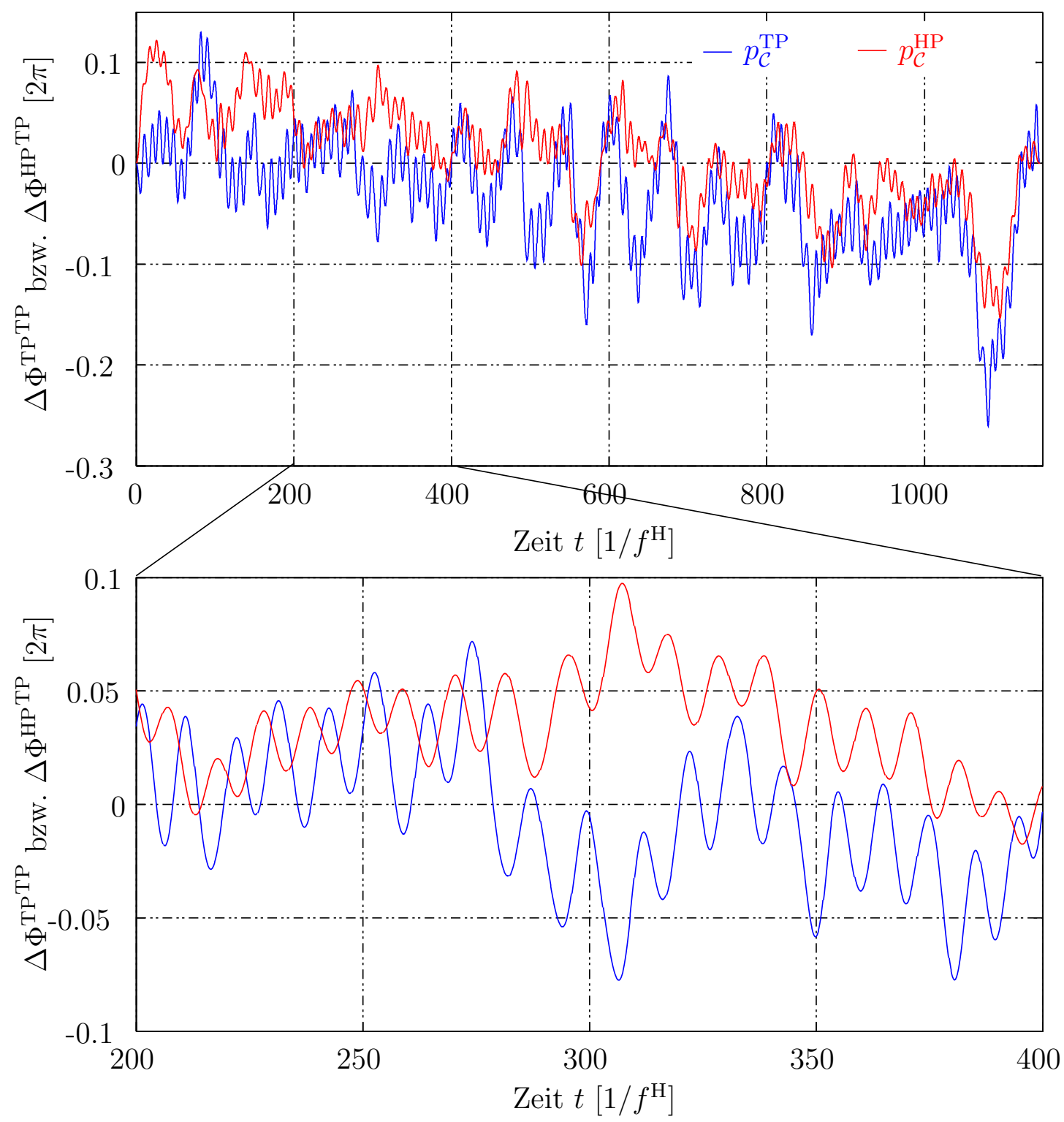

Abb. 4.21: Hauptfrequenzverhältnis 11/4. Oben: Phase ohne linearen Anteil und Vergrößerung. Parameter: $L / d=7.25, U_{\mathrm{D}}=5.36 \mathrm{~cm} / \mathrm{s}, d=4 \mathrm{~mm}, f^{\mathrm{H}}=1.75 \mathrm{~Hz}, \mathrm{Re}_{\mathrm{d}}=250$. 
Kapitel 4. Struktur und Dynamik der Moden

man zusätzlich die tieffrequenten Schwankungen der Beträge, so stellt man Ähnliches fest (hier nicht abgebildet). In Abb. 4.21 schwingen $\Delta \Phi^{\mathrm{TP} T \mathrm{PP}}$ und $\Delta \Phi^{\mathrm{HP}}{ }^{\mathrm{TP}}$ mit der Synchronisationsfrequenz $f^{\mathrm{H}} / 11$ und verhalten sich nahezu gegenphasig, was die Vergrößerung des Zeitausschnitts in Abb. 4.21 (unten) zeigt. Der 11/4 Rhythmus wird nicht immer streng eingehalten, was vermutlich durch die tieffrequentere Dynamik des Systems verursacht wird und nicht unbedingt auf Fluktuationen der Strömungsparameter zurückzuführen ist. Die gekoppelten Schwankungen machen deutlich, dass dem System eine noch tieferfrequentere Dynamik innewohnt als die schon erstaunlich lange Synchronisationsperiode.

Bei noch längeren Synchronisationsperioden, wie z.B. 17/6 in Abb. 4.22, oder sogar 47/16 wie in Abb. 4.23, sind die Abweichungen noch größer, so dass das wahre Synchronisationsverhältnis und dessen Schwankungen nur durch sehr lange Zeitreihen untersucht werden können. Die Varianz der Phasenverteilung zeigt Spitzen, die einen ersten Hinweis auf das Synchronisationsverhältnis liefern. Diese liegen nicht exakt auf den entsprechenden rationalen Frequenzverhältnissen auf Grund der nicht ganz rauschfreien Messdaten, der Auswertemethode (KLT und analytisches Signal) und vor allem der im System innewohnenden tieffrequenten Dynamik (siehe jeweils mittlere Grafik in Abb. 4.20, 4.22, 4.23). Die tieffrequenten Schwankungen der Differenzphase $\Psi\left(v^{\mathrm{H} / \mathrm{T}}, t\right)^{\mathrm{TP}}$ bestätigen das geschätzte rationale Verhältnis $v^{\mathrm{H} / \mathrm{T}}$ (siehe jeweils untere Grafik in Abb. 4.20, 4.22, 4.23).

Sehr erstaunlich ist das lange Gedächtnis der Strömung. Die 47 Schwingungen der hohen Mode entsprechen einer Periodendauer von $53 \mathrm{~s}$. Der Prozess, der das Frequenzverhältnis erzeugt, bzw. steuert, ist unbekannt. Eine systematische Untersuchung der Abhängigkeit des Frequenzverhältnisses von den Strömungsparametern ist aufwendig, da das Frequenzverhältnis in vielen Messungen - wie schon in Beispiel Abb. 4.16 diskutiert - nicht konstant ist. Für identische Parameterkonfigurationen ist die Dynamik der Moden stark unterschiedlich. So findet man in Abb. 4.24 für $L / d=8, d=4 \mathrm{~mm}, U_{\mathrm{D}}=5.36 \mathrm{~cm} / \mathrm{s}, R_{\mathrm{d}} \approx 240$ etwa eine Frequenz $f^{\mathrm{H}}$ der Mode II von 1.80-1.87 Hz. Die mittleren aus den Leistungsspektren oder aus dem Verhältnis der Gesamtphasendrehung von $p_{\mathcal{C}}^{\mathrm{TP}}$ und $p_{\mathcal{C}}^{\mathrm{HP}}$ bestimmten Frequenzverhältnisse $\left\langle v^{\mathrm{H} / \mathrm{T}}\right\rangle$ liegen zwischen 2.61 und 2.77. Für die Messung II und III ist $f^{\mathrm{H}}$ gleich, dennoch können sich die mittleren Frequenzverhältnisse unterscheiden.

Das Synchronisationsverhältnis kann bei etwa gleichen Parametern ebenfalls unterschiedlich sein. Für Messung I-III findet man annähernd eine 8:3 Synchronisation und für Messung IV 11:4. Die Tiefpass gefilterten Schwankungen der Phase zeigen wiederum die Frequenz $f^{\mathrm{H}} / m$ der Synchronisation an. Dabei sieht man, dass die Synchronisationsfrequenz $f^{\mathrm{H}} / m$ leicht schwankt. Das liegt hier insbesondere daran, dass $L / d=8$ gerade die Grenze von Systemzustand II zu Mode I darstellt.

Das Synchronisationsverhältnis $v^{\mathrm{H} / \mathrm{T}}=11 / 4$ stellt sich vor allem dann ein, wenn man von höheren Keilabständen her kommt. Wartet man einige Minuten (vorausgesetzt, der Systemzustand wechselt nicht zwischenzeitlich zu Mode I), so setzt sich $v^{\mathrm{H} / \mathrm{T}}=8 / 3$ durch. Vermutlich können auch andere Synchronisationsverhältnisse zu diesem $L / d$ erzeugt werden, indem man 

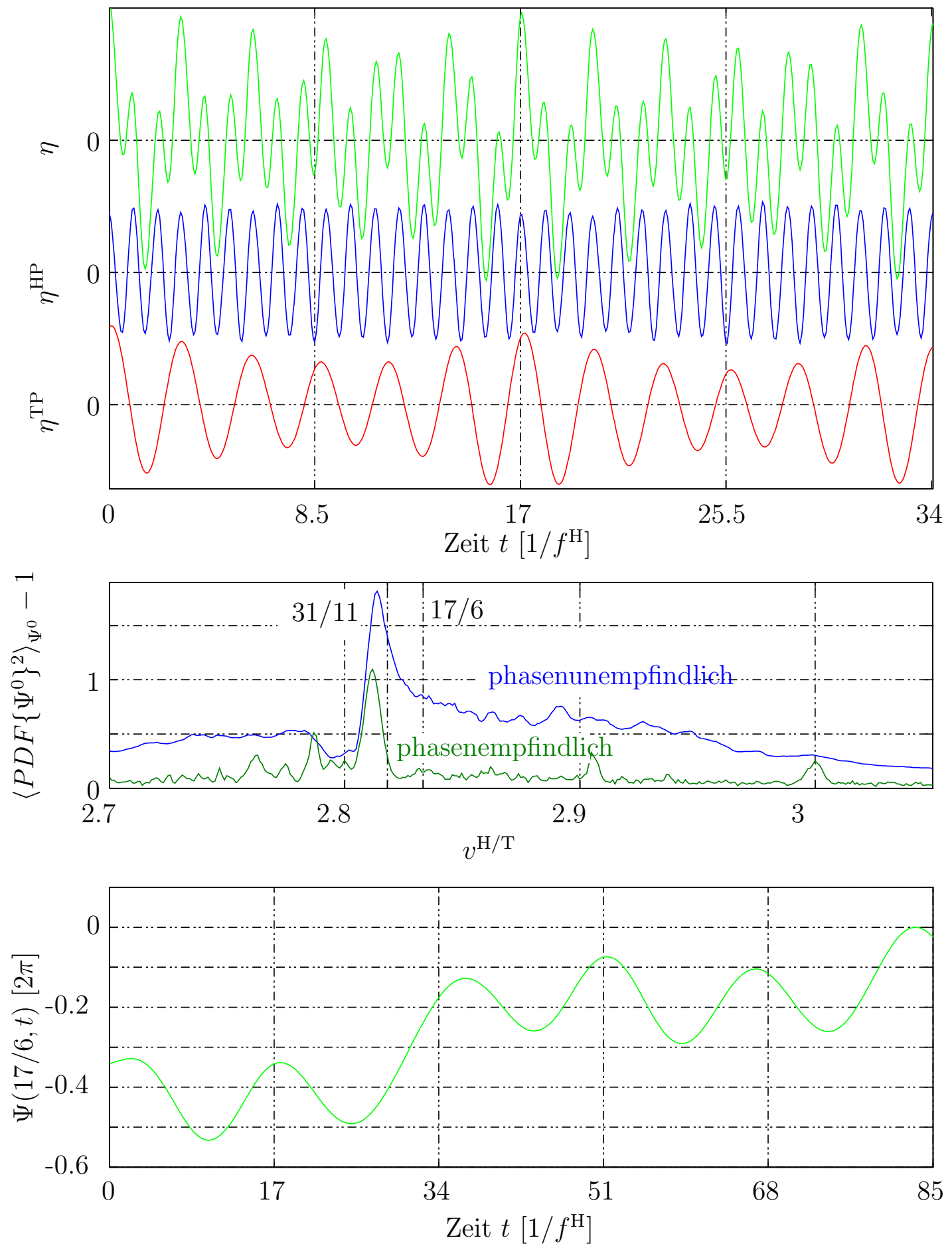

Abb. 4.22: Hauptfrequenzverhältnis 17/6. Oben: Zeitreihe. Mitte: Varianz der Phasenverteilung. Unten: $\arg \left(p_{\mathcal{C}}^{\mathrm{HP}}\right)-17 / 6 \cdot \arg \left(p_{\mathcal{C}}^{\mathrm{TP}}\right)$. Parameter: $L / d=7.75, U_{\mathrm{D}}=5.36 \mathrm{~cm} / \mathrm{s}, d=4 \mathrm{~mm}$, $f^{\mathrm{H}}=1.67 \mathrm{~Hz}$. 

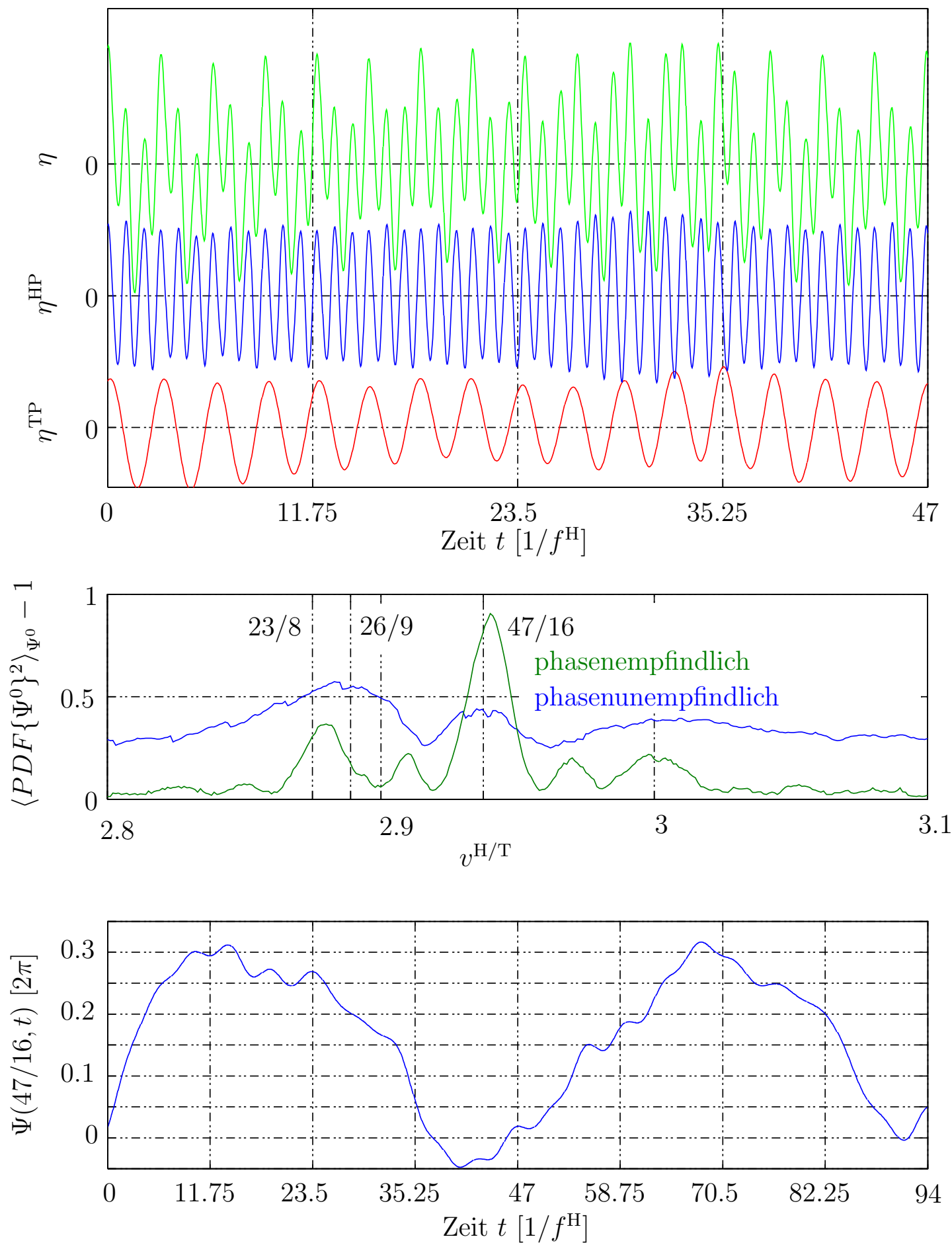

Abb. 4.23: Hauptfrequenzverhältnis 47/16. Oben: Zeitreihe. Mitte: Varianz der Phasenverteilung. Unten: $\arg \left(p_{\mathcal{C}}^{\mathrm{HP}}\right)-47 / 16 \cdot \arg \left(p_{\mathcal{C}}^{\mathrm{TP}}\right)$, Parameter: $L / d=11.25, U_{\mathrm{D}}=4.36 \mathrm{~cm} / \mathrm{s}$, $d=4 \mathrm{~mm}, f^{\mathrm{H}}=0.88 \mathrm{~Hz}$. 

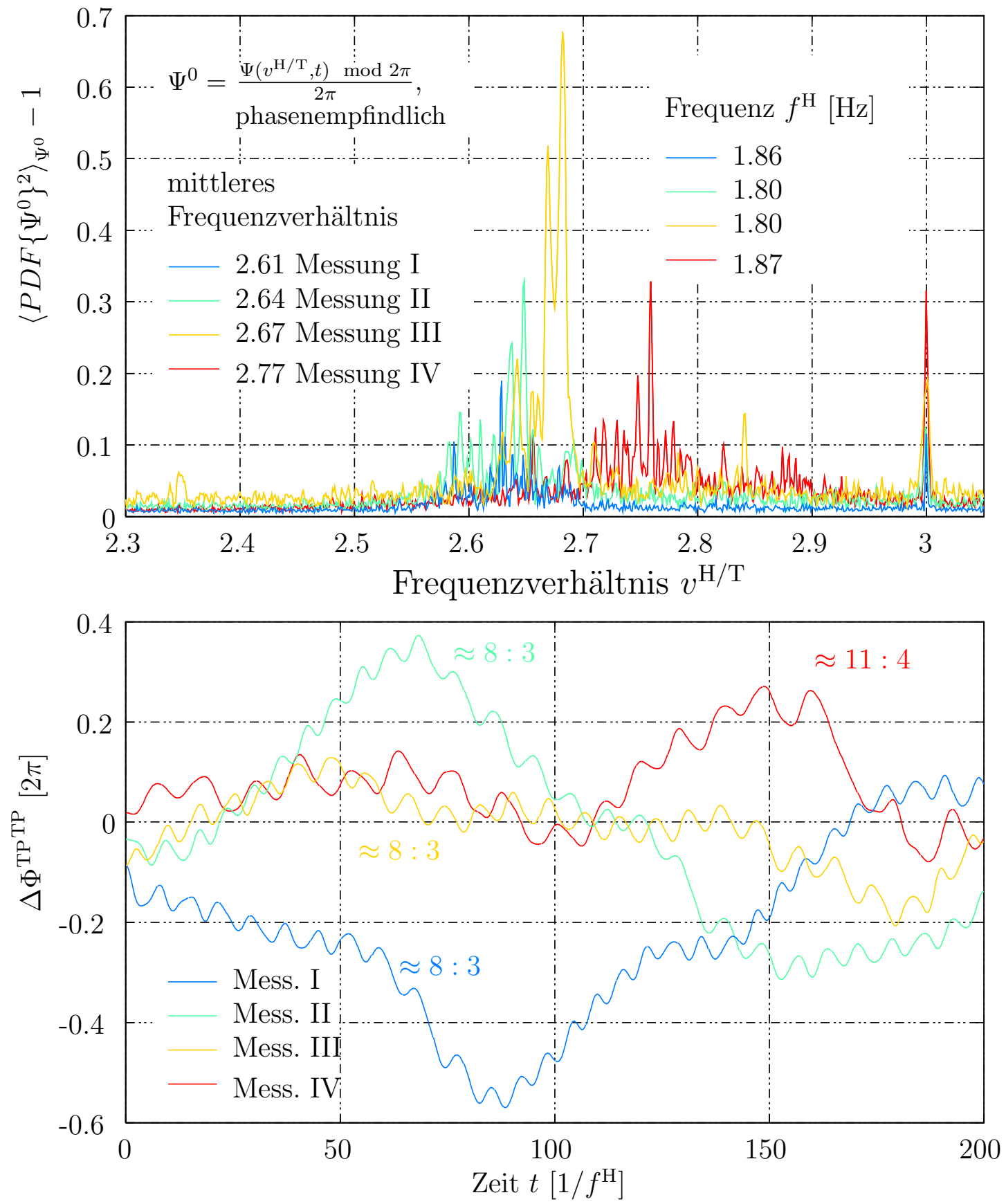

Abb. 4.24: Frequenzverhältnis und Phasenschwankungen bei Messungen mit sehr ähnlicher Parameterkonfiguration. Oben: Varianz der phasenemfindlichen Verteilung. Unten: Phase ohne linearen Anteil von $p_{\mathcal{C}}^{\mathrm{TP}}$. Parameter $L / d=7, U_{\mathrm{D}}=5.36 \mathrm{~cm} / \mathrm{s}, d=4 \mathrm{~mm}$, $f^{\mathrm{H}} \approx 1.8-1.87 \mathrm{~Hz}, \mathrm{Re}_{\mathrm{d}}=230$ 
Kapitel 4. Struktur und Dynamik der Moden

den Keil auf eine andere Position $L^{\prime} / d$ einstellt und dann den Keil wieder auf die Position $L / d$ zieht, nicht zu schnell, damit man die Strömungsstruktur nicht zerstört und nicht zu langsam, damit die Strömung sich nicht umstellen kann. Die Relaxationszeit kann mehrere Minuten betragen, wenn man die Strömungsstruktur nicht von außen durch große Aktuatorsignale oder schnelle Keilbewegungen zerstört. Ein weiteres Indiz für eine tieffrequente Dynamik des Systems ist die spontane Systemzustandsänderung, die im Hysteresebereich auftreten kann: Ein Systemzustand bleibt mehrere Minuten bestehen bevor er spontan in einen anderen Systemzustand übergeht. Auch die Stabilisierbarkeit der Strömung, mittels der im nächsten Kapitel beschriebenen Methoden, zeigt ein Langzeitverhalten: Ist die Strömung minutenlang stabilisiert worden, so stellt sich der stabile Zustand bei einer erneuten Stabililisierung schneller ein. All diese Indizien legen eine sehr tieffrequente Dynamik des Systems nahe, was ein entsprechend langes Gedächtnis voraussetzt.

Zur Erklärung dieses langen Gedächtnisses wird eine (Strömungs-)Struktur gesucht, die über eine lange Zeitdauer, verglichen mit der Schwingungsdauer $1 f^{\mathrm{H}}$, stabil ist. Die Wirbeligkeitsverteilung des schwingenden Strahls besteht aus stromab anwachsenden gegensinnig drehenden Gebieten, die durch den Keil getrennt werden. Die linksdrehenden Wirbel sammeln sich dann auf der rechten Seite und werden nur langsam stromab transportiert, da zusätzlich die Ausgleichsströmung (Abschnitt 3.3) die Abflussgeschwindigkeit erniedrigt. Daher können zu beiden Seiten des Keils großräumige Wirbelstrukturen entstehen, deren induziertes Feld die Position des Strahls verändern kann und damit die Strahl-Kanten-Wechselwirkung beeinflusst. In Abb. 4.18 lässt sich die weiter außen liegende Wirbelstruktur nicht erkennen, weil sie außerhalb des Bildes liegt. Da man die Wirbeldynamik nur anhand der Farbstoffverteilung im Kanal erahnen kann, müssten andere Messverfahren zur Aufklärung der Wirbelstruktur herangezogen werden. Eine Untersuchung der Wirbeldynamik in Bezug auf die Synchronisation könnte eventuell den Ursprung der tieffrequenten Dynamik klären.

\subsection{Erzwungene Modenübergänge}

Ein Systemzustand der Strahl-Kanten-Strömung kann mittels eines relativ kleinen Steuersignals in einen anderen Systemzustand überführt werden, sofern er bei den gewählten Parametern existenzfähig ist.

In Abb. 4.25 ist das Umschalten von Systemzustand II auf Mode I und umgekehrt dargestellt. Streut man die höhere Frequenz $f^{\mathrm{H}}$ von Systemzustand II ein, so geht die Mode I in Systemzustand II über und streut man die Frequenz $3 f_{\mathrm{I}}$ von Mode I ein, so geht der Systemzustand II in Mode I über. Man kann auch mit größerer Amplitude $f_{\mathrm{I}}$ einstrahlen, um den Systemzustand II in Mode I zu überführen, jedoch ist es nicht möglich mit $f^{\mathrm{T}}$, dem tieffrequenten Peak von Systemzustand II, den Systemzustand II aus Mode I heraus zu erhalten.

Das Ändern der Systemdynamik durch ein Steuersignal wird sich auch auf eine Regelung des 
Systems auswirken, die eine Stabilisierung zum Ziel hat. Eine der Regelung vorausgehende Systemerkennung, die den Systemzustand nicht ändern kann, sieht immer nur Teile des Phasenraums, je nachdem welcher Systemzustand gerade angeregt ist. Wird die Regelung zugeschaltet, so kann das System schon durch kleine Aktuatoramplituden in einen anderen Zustand übergehen und sich somit der Regelung entziehen, so dass eine erneute Systemerkennung notwendig wird. 


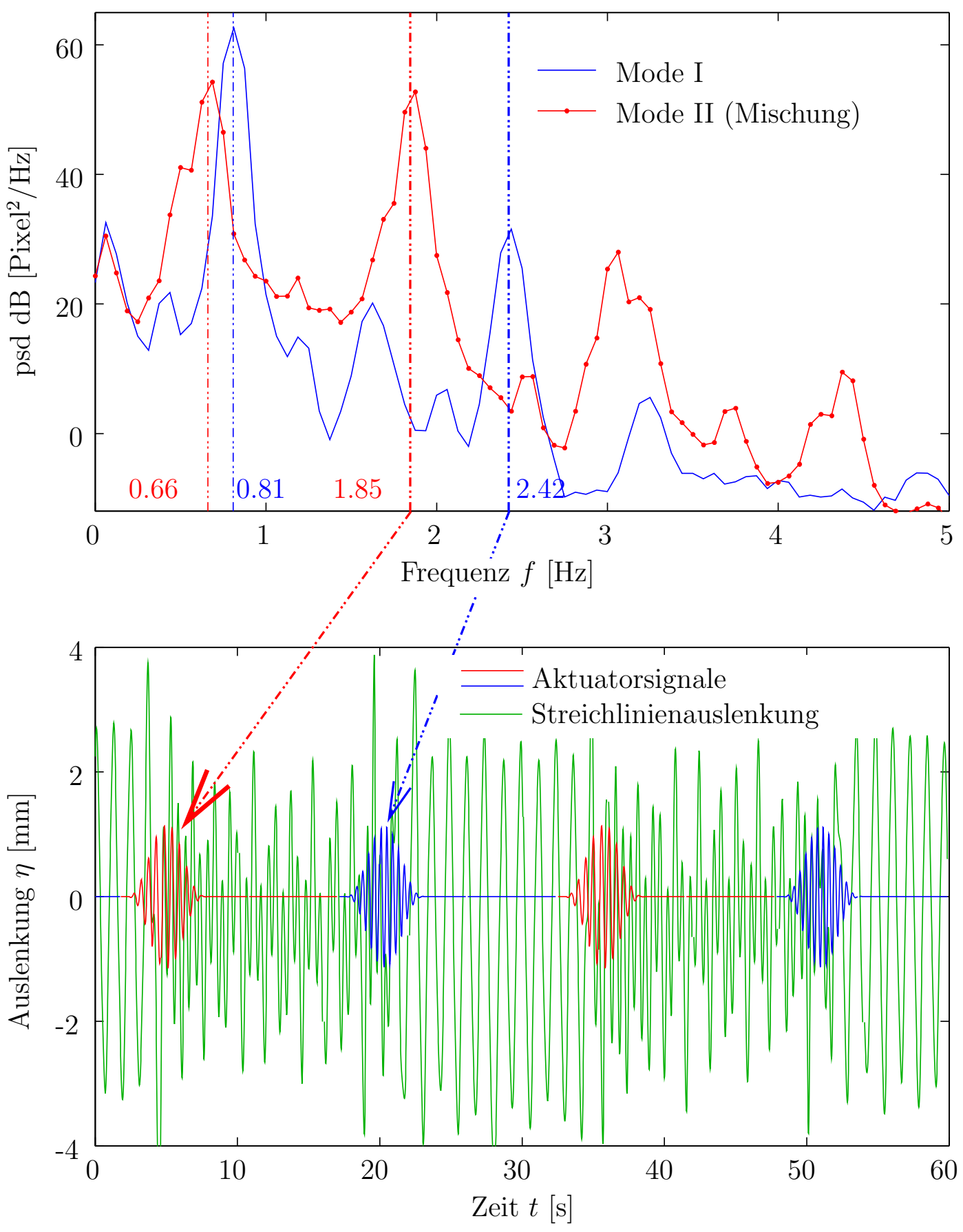

Abb. 4.25: Hysterese und Schalten der Moden. Oben: spektrale Leistungsdichte der Streichlinienauslenkung für Mode I und Mode II. Parameter: $L / d=7, \operatorname{Re}_{\mathrm{d}}=200, \max (s(t))=$ 150 Steps. 


\section{Kapitel 5}

\section{Regelung des Strahl-Kanten-Systems}

Die Dynamik des Strahl-Kanten-Systems soll durch eine Regelung beeinflusst werden. Dabei steht die Frage nach der Grenze der gezielten Beeinflussbarkeit im Vordergrund. Die Regelung soll hier eine Stabilisierung der Strömung bewirken. Da das System einer Regelung ausweichen kann, indem es höhere Freiheitsgrade aktiviert, ist ein sehr robuster Regler erforderlich. Eine Erweiterung der Freiheitsgrade kann dabei durch einen Systemzustandswechsel oder durch die Umstellung der Dynamik innerhalb eines Systemzustandes entstehen. Die im Kap. 4 gezeigten Eigenschaften, vor allem das lange Gedächtnis des Systems, machen es für den Regler schwierig, sich auf das System genau einzustellen, da durch die große Relaxationszeit lange Zeiten benötigt werden, bis die Systemdynamik durch die Regelung bestimmt wird bzw. alle autonomen Dynamiken kontrolliert sind.

Selbsterregt schwingende rückgekoppelte Systeme sind Systeme, deren offene Systemdynamik eine Verstärkung aufweist, die durch eine Nichtlinearität begrenzt wird. Es gibt leider kein allgemeines Rezept, ein solches System zu stabilisieren. Der Systemzustand muss aus dem Wirkungsbereich dieser Nichtlinearität herausgefahren werden. Falls dann zeitliche Schwankungen der Systemdynamik vernachlässigbar sind und die selbsterregte Schwingung fast stabilisiert ist, so dass die Nichtlinearität keine entscheidende Rolle mehr spielt, kann das System mittels linearer Regelungstheorie weiter stabilisiert werden und zudem können auch bestimmte dynamische Eigenschaften eingestellt werden, wie beispielsweise eine Übertragungsfunktion der Aktuatorauslenkung auf die Streichlinienauslenkung an einer bestimmten Messposition.

Im Folgenden werden verschiedene Regelstrategien diskutiert. Dabei ergibt sich - wie nicht anders zu erwarten - dass sich die Grenze der Stabilisierung immer weiter verschiebt, je ausgefeilter die Regelstrategie ist und je besser sie der Regelungsaufgabe und dem System angepasst ist. Zunächst stellt sich heraus, dass dies der Regelung des Strahl-Kanten-Systems mit steigender Reynoldszahl $\mathrm{Re}_{\mathrm{d}}$ und steigendem auf die Düsenbreite normiertem Düse-Keil-Abstand $L / d$ immer schlechter gelingt. In erster Näherung hat dies seine Ursache in der mit $L$ und $U_{\mathrm{D}}$ wachsenden konvektiven Verstärkung, die eine immer genauere Regelung des Systems verlangt. Daher lässt sich die Grenze einer zur Stabilisierung führenden Regelung in der $\operatorname{Re}_{\mathrm{d}}-L / d$-Ebene 
Kapitel 5. Regelung des Strahl-Kanten-Systems

angeben.

Für kleine Keilabstände $L / d$ und kleine Reynoldszahlen $\operatorname{Re}_{\mathrm{d}}$ soll zunächst davon ausgegangen werden, dass die Kenntnis der mittleren Systemdynamik ausreicht, um das System bezüglich der Stabilisierung zu charakterisieren. Das System kann unter dieser Voraussetzung mit einem zeitlich konstanten und von der Lage des aktuellen Orbits unabhängigen Regler auf den Fixpunkt gebracht werden. Entweder wird die mittlere Systemdynamik modelliert und ein dem entsprechender Regler entworfen, oder der passende Regler wird durch geschicktes Ausprobieren (per Hand, genetischer Algorithmen oder adaptiver Regelung) gefunden.

Der Regler muss mit wachsender Verstärkung bzw. Instabilität - und damit mit wachsender Gegenkopplung - immer genauer justiert werden, da das System durch Abweichungen von der optimalen Reglerrückkopplung entweder nicht mehr hinreichend stabilisiert oder sogar zur Instabilität bei anderen Frequenzen geführt werden kann. Die übersichtlichste Analyse eines zu regelnden Systems geht von der Übertragungsfunktion des offenen Systems aus. Man kann die physikalische Rückkopplungsschleife der Strahl-Kanten-Strömung jedoch nicht aufschneiden, um die Übertragungsfunktion des offenen Systems (bezüglich der physikalischen Rückkopplung) zu messen und einen Regler hinzufügen, der das geschlossene System stabilisiert. Dies ist eine starke Einschränkung für die sonst in der Regelungstechnik üblichen Entwurfsmuster für Regler. Außerhalb eines bestimmten Abstands vom Ursprung der $\operatorname{Re}_{\mathrm{d}}-L / d$-Ebene ist durch die dann notwendige genaue Einstellung der Gegenkopplung eine Stabilisierung mit Hilfe eines festen Reglers nicht mehr möglich. Außerdem ist die Systemdynamik im Grenzzyklus dann zu weit von der Dynamik des stabilisierten Zustands entfernt, so dass es notwendig wird, einen Regler zu entwerfen, der von dem aktuellen Orbit im Phasenraum abhängig ist. Das Problem, das durch die Wechselwirkung zwischen Regler und System in diesem Fall entsteht, wird im Folgenden beschrieben.

Um das System aus dem Grenzzyklus heraus zu stabilisieren, kann man versuchen, die Systemdynamik durch kleine Testsignale kennenzulernen. Diese Information wird über viele Perioden des Grenzzyklus gemittelt, um so eine mittlere Systemdynamik in der Nähe des Orbits abzuschätzen. Dieser Orbit kann durch Hinzufügen eines Reglers gezielt verändert werden, so dass die Schwingungsamplitude langsam kleiner wird. Im nächsten Schritt wird der neue Phasenraum des geregelten Systems in der Nähe des neuen Orbits analysiert und das System auf diese Weise auf immer kleineren Grenzzyklen bis in den Fixpunkt geregelt. Das Adaptionssystem erfasst dabei die linearisierte gemittelte Dynamik in der Nähe des aktuellen Orbits. Dies reicht nicht mehr aus, wenn die Abweichung von der mittleren Systemdynamik wesentlich für den Erfolg der Regelung ist. Das Regelssystem muss dann erkennen, an welchem Punkt sich das System im Phasenraum befindet, in Abhängigkeit von diesem Punkt die Systemdynamik analysieren und eine dazu passende nichtlineare Regelstrategie errechnen.

Die hier beobachtete Dynamik des Strahl-Kanten-Systems fluktuiert langsam verglichen mit der Schwingungsdauer des Systems (für $L / d<15$ ). Die Dynamik des Systems, beispielswei- 
se in Systemzustand II, hat abgesehen von der „hochfrequenten“ Dynamik der selbsterregten Schwingung, einen tieffrequenten Anteil, dessen Zeitskala mit der Periodenlänge der Synchronisation und einer Größenordnung darunter vergleichbar ist (siehe Kap. 4.7.2). Zwar werden die für die Synchronisation wichtigen Mechanismen durch die Regelung gestört, aber der letztgenannte tieffrequente Anteil der Dynamik bleibt wahrscheinlich für die Stabilisierung relevant. Das Adaptionssystem muss oberhalb einer gewissen Schleifenverstärkung diesem tieffrequenten Anteil der Systemdynamik folgen können, ansonsten kann aufgrund der nicht genau eingestellten Gegenkopplung eine Stabilisierung nicht erreicht werden. Das Adaptionssystem kann der Systemdynamik folgen, solange die typischen Zeitskalen der Systemdynamik lang gegenüber der Adaptionszeit des Reglers sind. Die mit wachsender Schleifenverstärkungen notwendige genaue Einstellung des Reglers verlängert die Adaptionszeit. Eine genaue Adaption des Reglers und ein schnelles Folgeverhalten schließen sich dann gegenseitig aus, so dass nur noch eine vorherige detaillierte Modellierung der geregelten Systemdynamik weiter hilft.

Im Folgenden werden zunächst einfache, dann besser angepasste und schlussendlich adaptive Regler für das Strahl-Kanten-System dargestellt und ihre Grenzen aufgezeigt.

\subsection{Phasenverschiebungsregler}

Die einfachste Art einer Regelung (Abb. 5.1) besteht darin, die an der Stelle $x_{\text {Regel }}$ gemessene Streichlinienauslenkung $\eta\left(x_{\text {Regel }}, t\right)$ verstärkt und verzögert (Verstärkungsfaktors $K$. Verzögerungszeit $\tau$ ) auf die Strömung mit entgegengesetztem Vorzeichen zurückzukoppeln. Die Impulsantwort dieses Reglers hat die Form $K \delta(t-\tau)$ und entspricht damit einem digitalen Filter, dessen Koeffizienten bei der Verschiebung $\tau$ den Wert $K$ und sonst den Wert null haben. Die Parameter $K, \tau$ werden per Hand optimiert, so dass die physikalische Rückkopplung durch die Gegenkopplung mit den Parametern $K_{\mathrm{opt}}, \tau_{\mathrm{opt}}$ genügend kompensiert wird, um die selbsterregte Schwingung zu unterdrücken. Dabei kann die Verzögerung auch direkt durch Verschieben der Messposition eingestellt werden. Beim Einschalten der Regelung mit den optimierten Parametern wird der Verstärkungsfaktor $K$ langsam erhöht, bis er $K_{\text {opt }}$ erreicht (Fading). Bei zu schnellem Einschalten kann eine Regelungsmode destabilisiert werden, und das System schwingt - durch die Strahlinstabiliät gesättigt - in einem neuen Grenzzyklus. Erhöht man den Verstärkungsfaktor langsam bis auf $K_{\mathrm{opt}}$, so wird eine Stabilisierung, also der Fixpunkt, erreicht. Um die reglerinduzierte Instabilität zu vermeiden, wird zusätzlich eine Sättigungsfunktion in die Gegenkopplungsschleife eingefügt. Damit lässt sich das System sehr schnell einschalten, da das Regelsignal beschränkt bleibt und somit dem Systemzustand angepasst ist. Um die Dynamik des geregelten Systems zu charakterisieren und die Güte der Stabilisierung zu messen, wird das System mit einem kleinen breitbandigen Testsignal $s(t)$ angeregt, das hier ein stochastisches Rauschsignal ist. Die Antwort des Systems auf $s(t)$ wird durch das Leistungsspektrum der Streichlinienauslenkung $\eta\left(x_{\text {Mess. }}, t\right)$ an der Position $x_{\text {Mess. }}$ charakterisiert, 


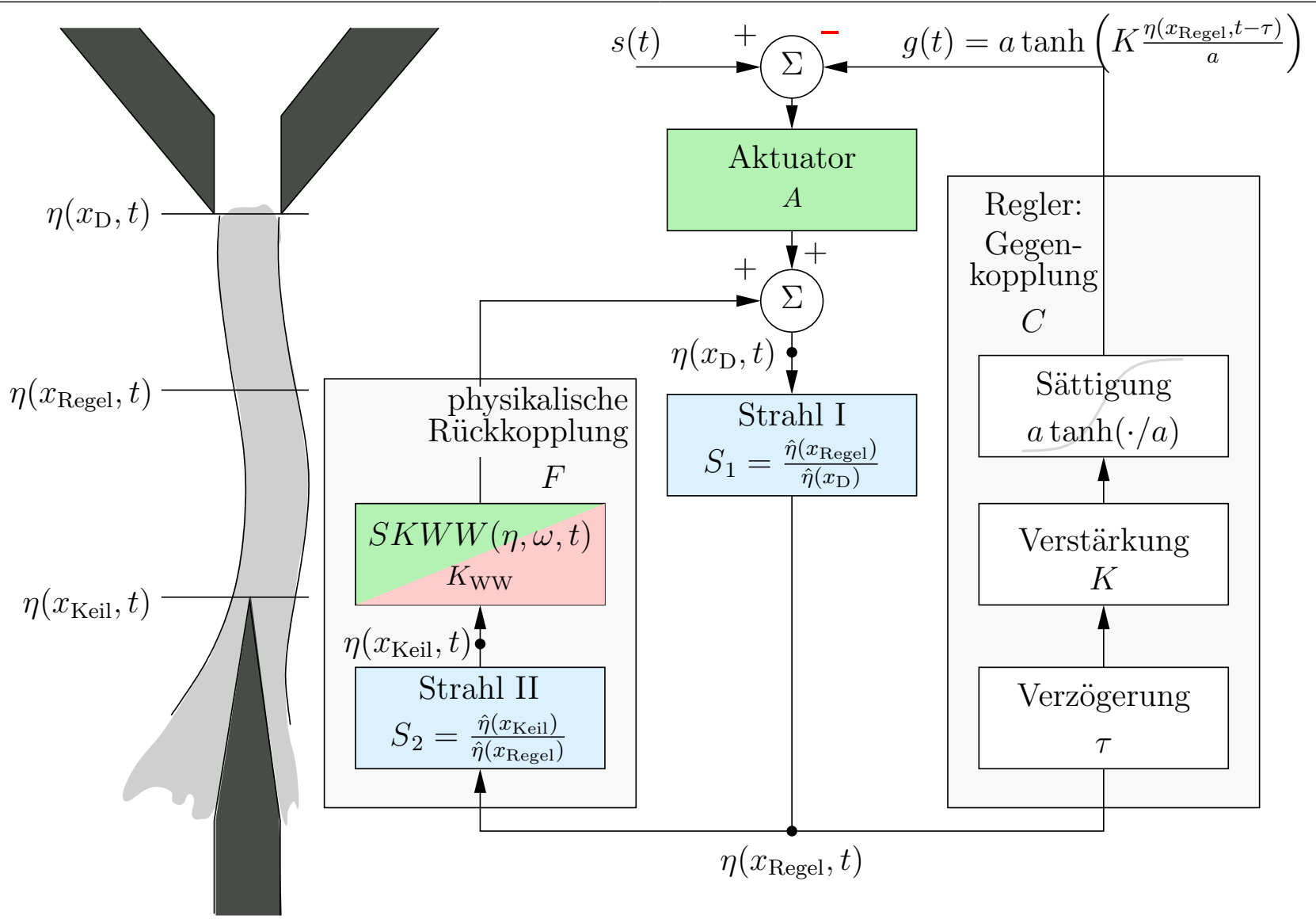

Abb. 5.1: Gegenkopplung mit verzögertem und verstärktem Messsignal.

die nicht der Position $x_{\text {Regel }}$ entsprechen muss, von der aus geregelt wird. Hier wird das Leistungsspektrum herangezogen und nicht die Übertragungsfunktion $H^{1}$, da im Gegensatz zur Übertragungsfunktion auch die nicht mit dem Signal $s(t)$ kohärenten Anteile sichtbar sind. Der Vergleich der Leistungsspektren der natürlichen Mode und des geregelten Systems zeigen Spitzen bei Frequenzen $f$, die den Polen $(i 2 \pi f-\sigma)$ der Übertragungsfunktion $H$ in der komplexen Ebene nahekommen und damit die mögliche Instabilität des Systems anzeigen. Bei diesen Frequenzen können leicht Regelungsmoden angeregt werden. Das System ist daher umso stabiler je weniger ausgeprägt die Spitzen im Leistungsspektrum vorhanden sind.

Die Leistungsspektren des geregelten Systems sind in Abb. 5.2 für zwei verschiedene normierte Keilabstände $L / d$ zusammen mit den unter Rauschen gemessenen Leistungsspektren des

\footnotetext{
${ }^{1}$ Die Übertragungsfunktion $H:=\left.\hat{\eta}\left(x_{\text {Mess. }}\right)\right|_{s} / \hat{s}$ des Systems bezieht sich auf den zu $s(t)$ kohärenten Anteil $\left.\hat{\eta}\left(x_{\text {Mess. }}\right)\right|_{s}$ und kann durch Kreuzkorrelation ermittelt werden:

$$
H:=\mathcal{F}\left\{\left\langle s(t-\tau) \cdot \eta\left(x_{\text {Mess. }}, t\right)\right\rangle_{t}\right\} / \mathcal{F}\left\{\left\langle s(t-\tau) \cdot s\left(x_{\text {Mess. }}, t\right)\right\rangle_{t}\right\} .
$$
}




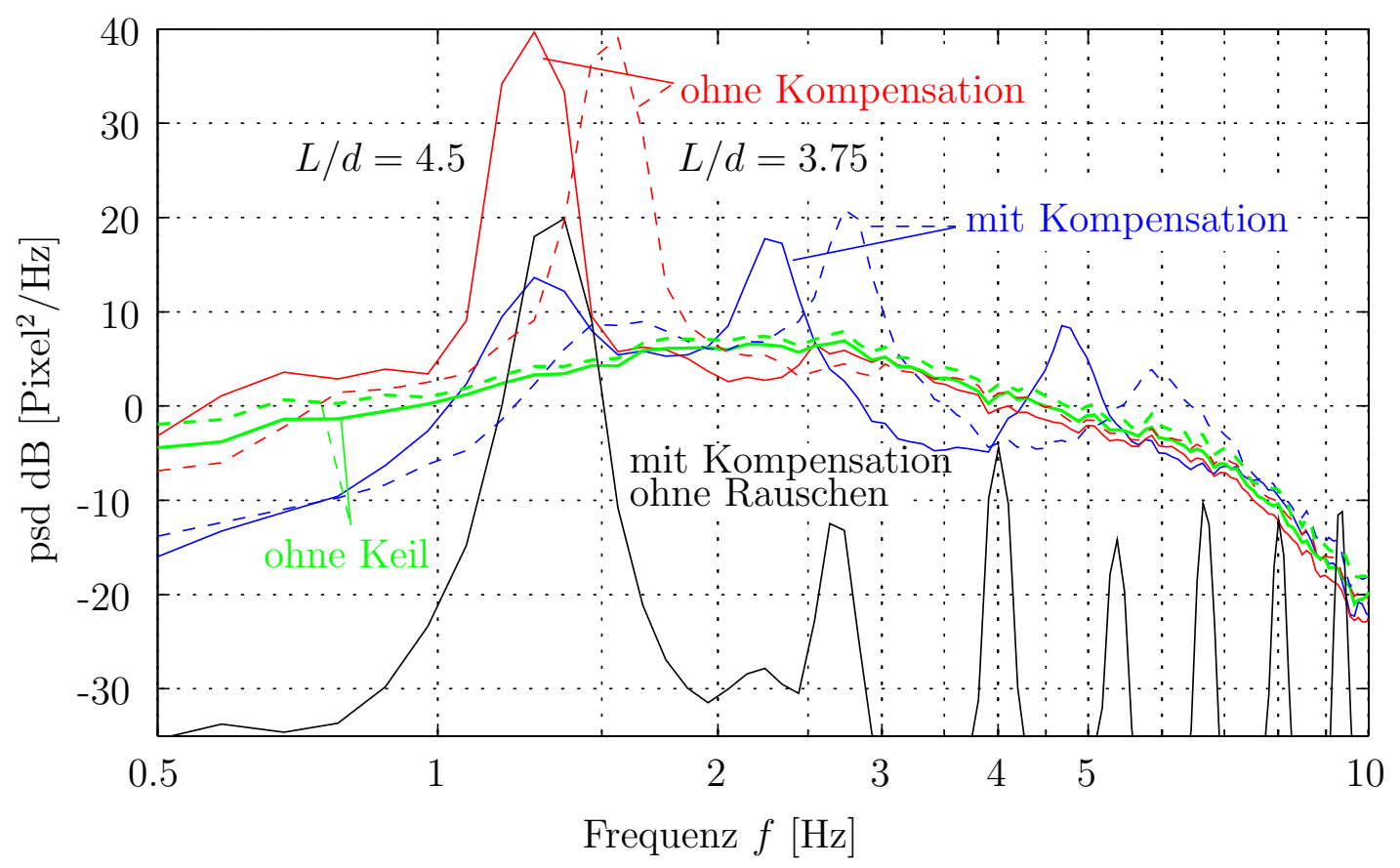

Abb. 5.2: Grenze der Regelung mit einem Koeffizienten. Messung des Leistungsspektrums an einer typischen Messposition mit Rauschanregung: $\left|s^{\max }\right|=300$ Steps, $\operatorname{Re}_{\mathrm{d}}=215$, $T=21^{\circ} \mathrm{C}, U_{\mathrm{D}}=5.36 \mathrm{~cm} / \mathrm{s}$. Die qualitativen Eigenschaften der hier gezeigten Ergebnisse sind unabhängig von der Messposition.

ungeregelten Systems und der konvektiven Instabilität des Systems ohne Keil verglichen. Dabei wurde die veränderte Effektivität des Aktuators aufgrund der durch den Keil verringerten Querschnittsfläche berücksichtigt, indem das Leistungsspektrum der konvektiven Instabilität mit einem aus der Potenzialtheorie stammenden Faktor multipliziert wurde. Mit einer guten Regelung sollte die spektrale Leistungsdichte des geregelten Systems das Niveau der konvektiven Instabilität erreichen, oder sogar unterschreiten (siehe Abschnitt 5.3).

Für $L / d=4.5$ schwingt das geregelte System leicht auf der Frequenz 1.3 Hz (Abb. 5.2) und das Leistungsspektrum ohne Rauschen (schwarze Linie) zeigt dort eine deutlich höhere Spitze als das mit Rauschen angeregte geregelte System (blaue Linie), d.h. durch Überlagerung des Rauschens wird die Schwingung kleiner und geht bei größeren Anregungsamplituden im Rauschen unter. Die wahrscheinlichste Ursache für diesen Effekt sind die durch das Rauschen veränderte Strahl-Kanten-Wechselwirkung und die Verbreiterung des Strahls (siehe Abschnitt B.1.2), die eine Abnahme der konvektiven Verstärkung zur Folge haben. Die unter Rauschen gemessene spektrale Leistungsdichte der ungeregelten Strahlschwingung wird durch die Regelung um etwa $20 \mathrm{~dB}$ abgesenkt. Die Leistungsdichte des Systems ohne Keil wird für tiefe Frequenzen im kompensierten Zustand sogar unterschritten, da das Rauschsignal durch die Gegenkopp- 


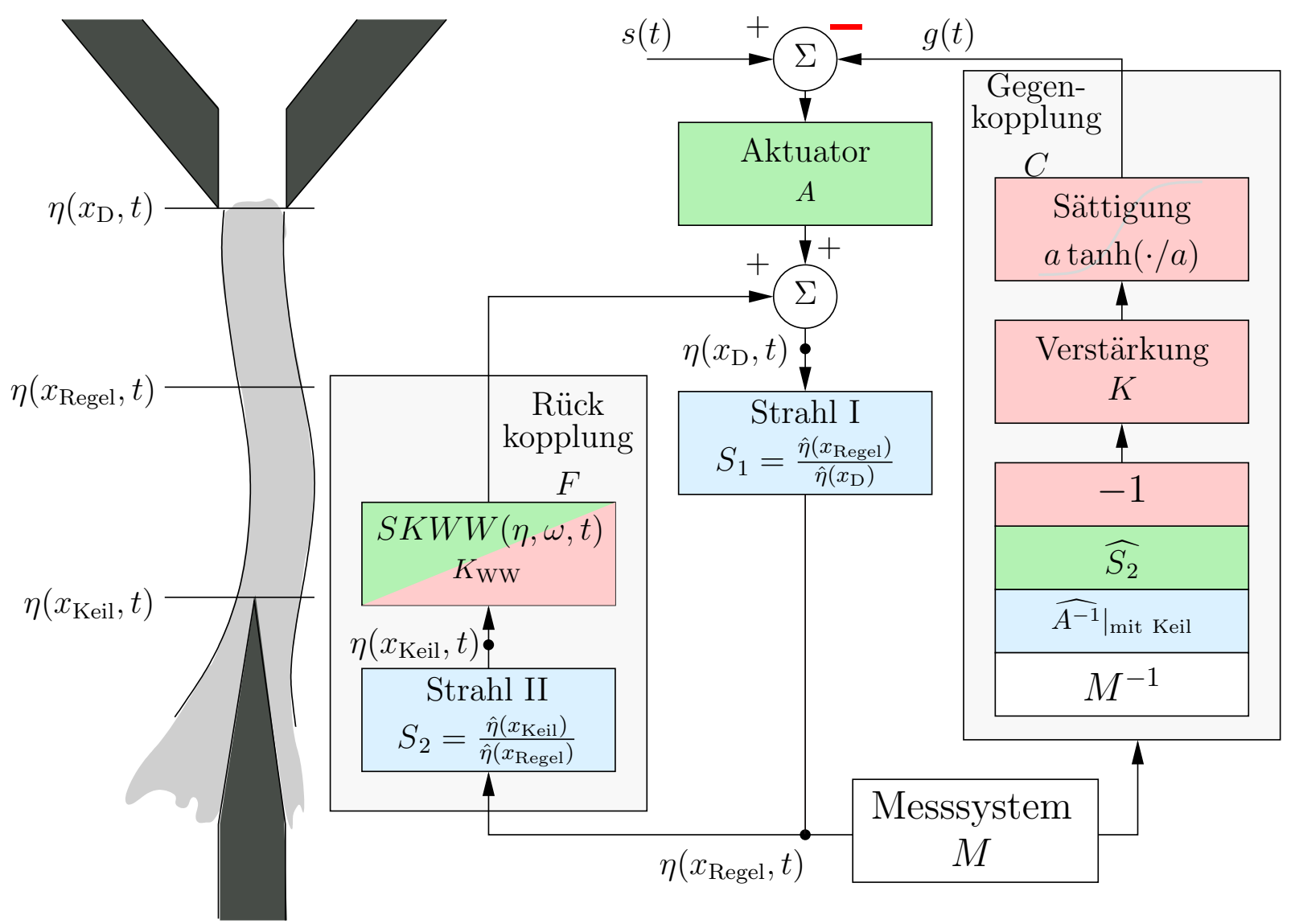

Abb. 5.3: Modell mit konstanter Keilübertragungsfunktion $-\boldsymbol{k}$.

lung zeitverzögert und verstärkt zu sich selbst addiert wird und so für tiefe Frequenzen (kleine Phasendrehung) die geschlossene Übertragungsfunktion des Systems näherungsweise eine Interferenz entstehen lässt.

Bei dieser sehr einfachen Gegenkopplung ist für $\operatorname{Re}_{\mathrm{d}}=215$ bei $L / d \approx 4.5$ und für $\operatorname{Re}_{\mathrm{d}}=120$ bei $L / d \approx 5$ die Grenze der Stabilisierbarkeit erreicht.

\subsection{Regelung basierend auf dem Modell einer frequenz- unabhängigen Keilübertragungsfunktion}

Die selbsterregte Schwingung des Strahl-Kanten-Systems zeigt eine Phasendifferenz von $-\pi$ zwischen der Auslenkung an der Düse und der Auslenkung am Keil (Abb. 4.3, Abb. 4.4). Diese ist für alle Moden und damit auch für alle relevanten Frequenzen relativ konstant. Daher liegt es nahe eine Gegenkopplung zu modellieren, deren Phasenbeziehung zwischen der Auslenkung am Keil und der Auslenkung an der Düse ebenfalls gerade $-\pi$ ist. Aus Laufzeitgründen ist es nicht 
möglich, die Streichlinienauslenkung, die am Keil gemessen wird, direkt zur Kompensation zu benutzen. Aufgrund der Zeitverzögerung von $60 \mathrm{~ms}$ zwischen Bildaufnahme und Aktuatoransteuerung und der Gruppenlaufzeit von $A$ zeigt $A^{-1}$ wesentliche Anteile für negative Zeiten, ist also akausal. Daher wird das Messsignal $\eta\left(x_{\text {mess }}, t\right)$ mittels einer ohne Keil geschätzten Übertragungsfunktion $\widehat{S_{2}}(f)=\left.\frac{x_{\text {Keil }}}{x_{\text {mess }}}(f)\right|_{\text {ohneKeil }}$ auf die Keilposition $x_{\text {Keil }}$ extrapoliert. Das kausale Gegenkopplungsfilter $\left.\mathcal{F}^{-1}\left\{\widehat{S_{2}} \widehat{A^{-1}}\right\}\right|_{\mathrm{t}>0}$ kann im Frequenzbereich aufgrund der hohen Bandbreite von $A$ problemlos berechnet werden. Die unbekannte Verstärkung der Strahl-Kanten-Wechselwirkung wird durch einen Verstärkungsfaktor $K$ modelliert und das Gegenkopplungssignal $g(t)$ nicht-linear begrenzt:

$$
g(t)=a \tanh \left(K \cdot \eta\left(x_{\text {Regel }}, t\right) *\left[-\left.\mathcal{F}^{-1}\left\{\widehat{S_{2}} \widehat{A^{-1}}\right\}\right|_{t>0}\right] / a\right) .
$$

Der Verstärkungsfaktor $K$ wird nun per Hand so lange verändert, bis eine optimale Stabilisierung des Systems erreicht ist.

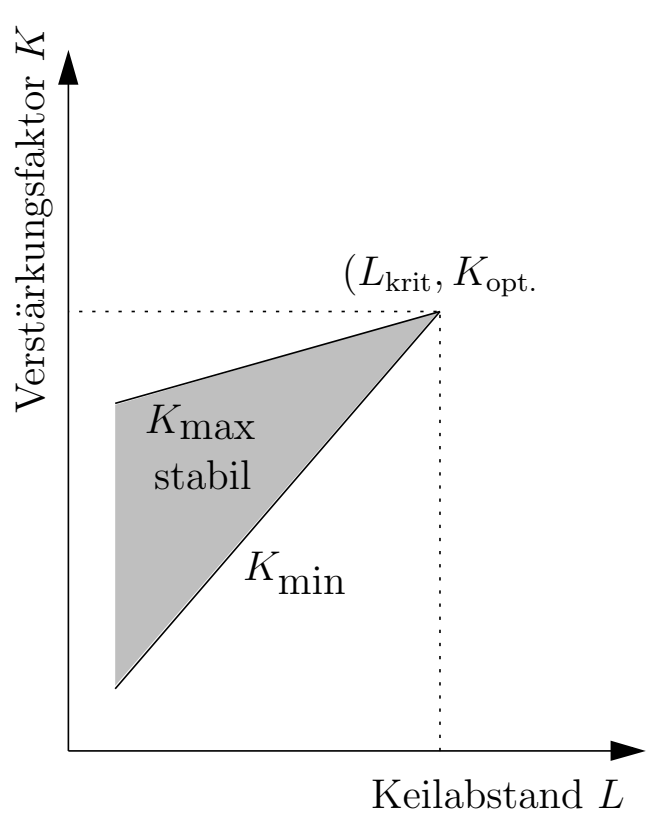

Abb. 5.4: Stabilitätsbereich bezüglich des Verstärkungsfaktors. jeweils etwa gleichen Phasenlage eingeschaltet werden konnte (Abb. 5.5 (unten)). Zu Beginn der Messung sind die Systemdynamiken fast identisch, da die nichtlineare Begrenzung den Verstärkungsfaktor zunächst absenkt. In beiden Fällen wird die Mode I zunächst unterdrückt. Für den kleineren Verstärkungsfaktor kann danach die reglerinduzierte Mode nicht anwachsen und das System wird stabilisiert, während für den größeren Verstärungsfaktor die reglerinduzierte Mode anschwingt und das Gesamtsystem somit instabil wird.

Abbildung 5.5 (Mitte) zeigt, dass für einen ganzen Bereich von Verstärkungsfaktoren $K_{\min }<$ $K<K_{\max }$ eine befriedigende Stabilisierung der Strömung erreicht wird. Dieser Bereich wird 

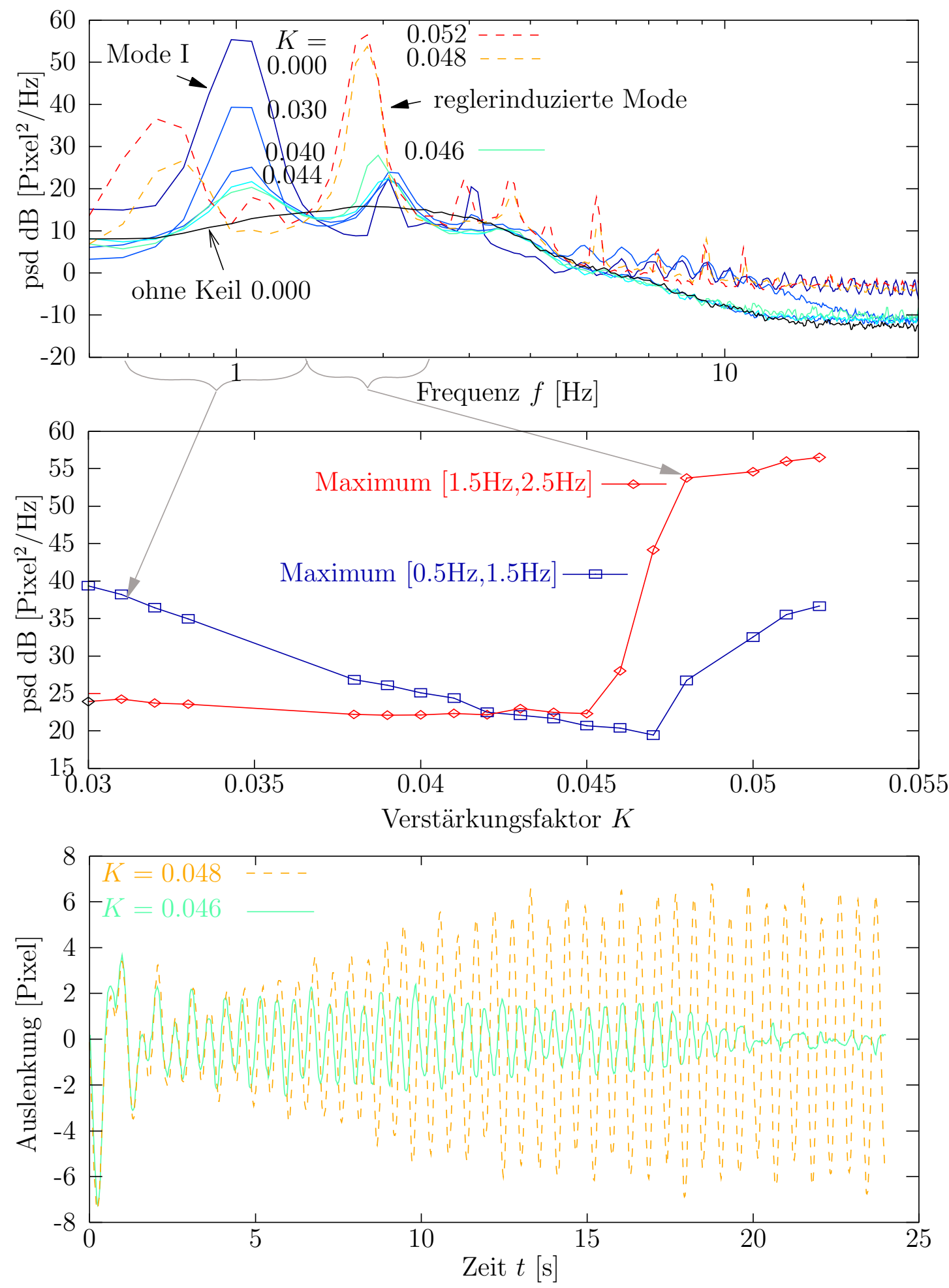

Abb. 5.5: Güte der Stabilisierung in Abhängigkeit von dem Verstärkungsfaktor $\boldsymbol{K}$. Parameter: $\operatorname{Re}_{\mathrm{d}}=200, U_{\mathrm{D}}=4.93 \mathrm{~cm} \cdot \mathrm{s}^{-1}, L / d=5, T=20.8^{\circ} \mathrm{C}$, Rauschanregung $\left|s^{\max }\right|=100$ Steps. 

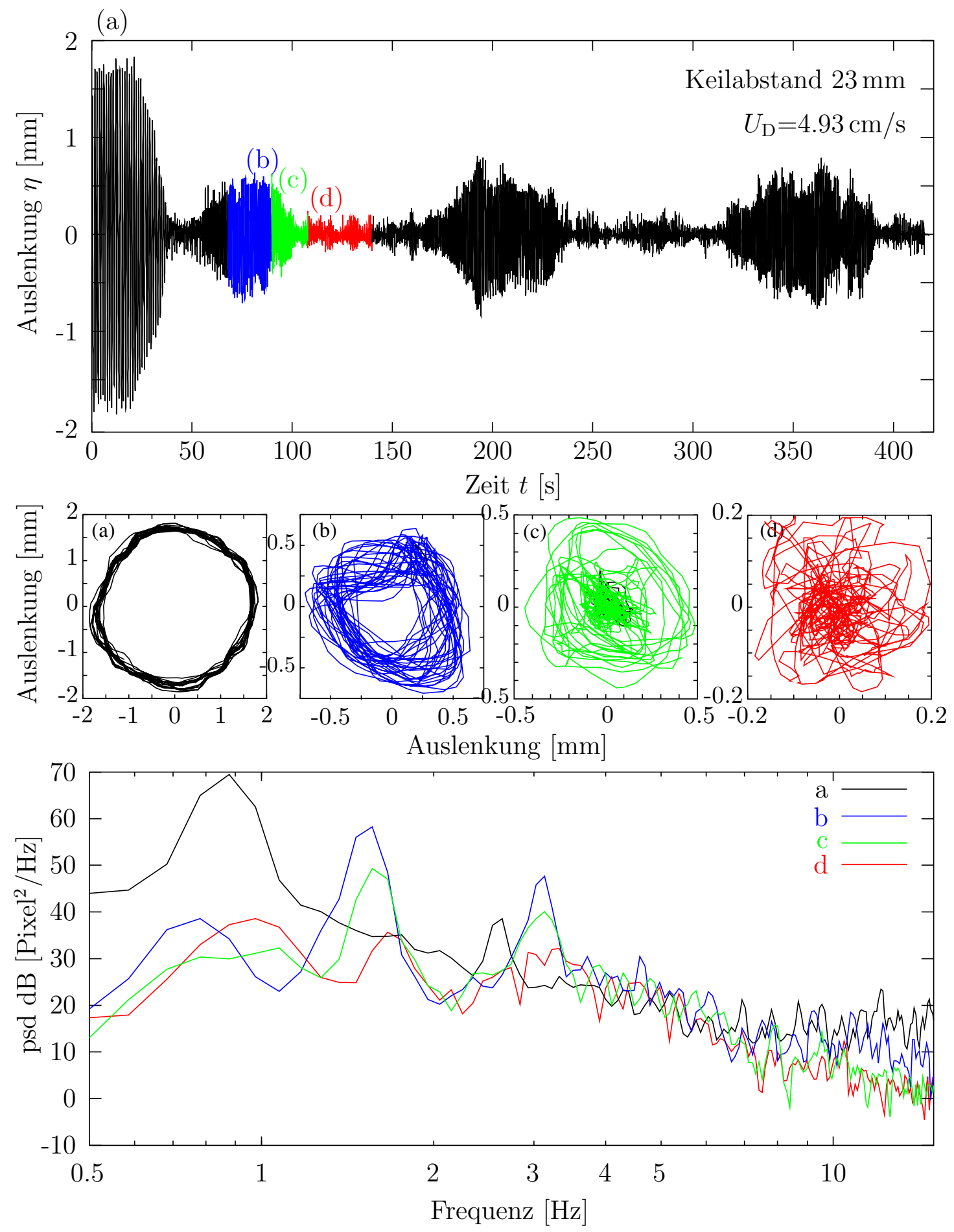

Abb. 5.6: Systemzustände unter Regelung nach Modell II. Oben: Zeitreihe der Stabilisierung (a) Schwingung ohne Regelung (b) Zustand höherer Frequenz, (c) Übergang, (d) „Fixpunkt". Mitte: Bild der Zustände in Verzögerungskoordinaten in unterschiedlicher Skalierung. Unten: Leistungsspektren. Parameter: $\operatorname{Re}_{\mathrm{d}}=200, U_{\mathrm{D}}=4.93 \mathrm{~cm} / \mathrm{s}, L / d=5.75, T=20.8^{\circ} \mathrm{C}$. 
Kapitel 5. Regelung des Strahl-Kanten-Systems

aber mit wachsendem Düse-Keil-Abstand kleiner, und es gibt schließlich einen kritischen Abstand, bei dem der minimal notwendige und der maximal zulässige Verstärkungsfaktor ineinander übergehen, wie in Abb. 5.4 verbildlicht ist.

Die Zeitreihe der Strahlauslenkung in Abb. 5.6 zeigt eine Stabilisierung bei diesem kritischen Abstand für den optimal eingestellten Verstärkungsfaktor $K_{\mathrm{opt}}$. Das geregelte System wurde mit einem sehr kleinen, breitbandigen Rauschsignal angeregt, um die Güte der Stabilisierung zu testen. Bei konstantem Verstärkungsfaktor $K_{\mathrm{opt}}$ kann sich das System in verschiedenen, sich zeitlich abwechselnden Zuständen befinden. Nach dem Abschnitt (a) $(0 s<t \leq 20 s)$, in dem das System ungeregelte selbsterregte Schwingungen ausführt, wird der Verstärkungsfaktor $K$ für $(20<t<40)$ langsam auf $K_{\text {opt }}$ erhöht, so dass die Amplitude der selbsterregten Schwingung abnimmt. Der Bereich der möglichen $K$, die die Strömung stabilisieren, ist hier an der Grenze der Stabilisierbarkeit sehr klein, und der stabilisierte Zustand bleibt nicht andauernd bestehen. Das System pendelt unregelmäßig zwischen dem stabilisierten Zustand (d) und dem Zustand (b), der der leicht veränderten höheren Mode II des ungeregelten Systems entspricht. Die Schwankungen der einzelnen Systemzustände (a), (b) und (d) sind in Abb. 5.6 (Mitte) durch Verzögerungskoordinaten veranschaulicht. Die absoluten Schwankungen (also die mittlere Abweichung vom mittleren Orbit) sind aufgrund der Rauschanregung annähernd gleich groß.

Die Grenze der Modellierung durch eine frequenzunabhängige Keilübertragungsfunktion liegt für $\operatorname{Re}_{\mathrm{d}}=200$ bei $L / d \approx 6$.

\subsection{Adaptive Regelung}

Abgesehen von der Steuerung des Verstärkungsfaktors während des Einschaltvorgangs, um das System langsam aus dem Grenzzyklus in den Fixpunkt zu ziehen, basieren die bisher dargestellten Stabilisierungsmethoden auf zeitlich konstanten Gegenkopplungsfiltern. Mit wachsendem $L / d$ wird der Bereich der zur Stabilisierung führenden Parameter kleiner und die Gegenkopplung muss deutlich langsamer eingeschaltet werden. Wegen der wachsenden Verstärkung der Rückkopplung muss die Gegenkopplung mit höherer Genauigkeit eingestellt werden. Da die Modelle der Rückkopplung alle sehr wenig über den Frequenzgang der linearisierten StrahlKanten-Wechselwirkung, hier auch Keilübertragungsfunktion genannt, aussagen, muss die Gegenkopplung möglichst modellfrei, also adaptiv, eingestellt werden. Auch eine Berücksichtigung der sich zeitlich ändernden Systemdynamik wird erst ab einem gewissen $L / d$ für die Stabilisierung wesentlich.

Die adaptive Regelung kann daher genutzt werden:

- um die Gegenkopplung genau einzustellen, da kein Modell der Rückkopplung verfügbar ist, 


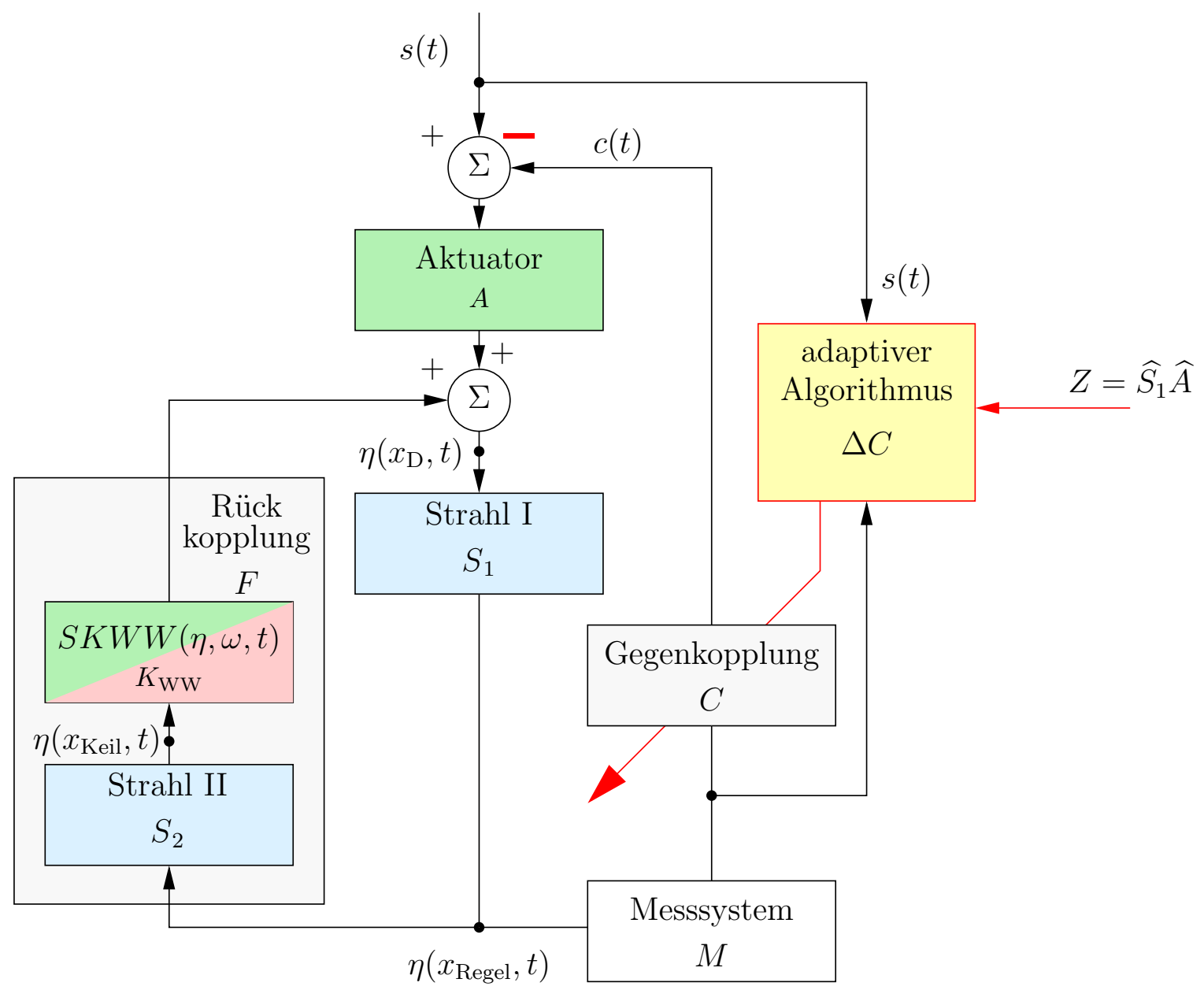

Abb. 5.7: Adaptive Regelung.

- um Schwankungen der Systemdynamik zu folgen, sofern das Folgeverhalten des adaptiven Reglers schnell genug ist,

- um die Nichtlinearität des im Grenzzyklus schwingenden Systems zu durchfahren und die eventuell auf verschiedenen Orbits notwendigen unterschiedlichen Gegenkopplungen quasistationär einzustellen und so mit einer linearen Regelung in Abhängigkeit von dem aktuellen mittleren Orbit das System zu stabilisieren.

\subsubsection{Entwurf des adaptiven Regelalgorithmus}

Denkt man sich die physikalische Rückkopplung $F$ durch die Regelung mit der Gegenkopplung $C=C_{\text {opt }}$ in Abb. 5.7 kompensiert, so ist die Übertragungsfunktion bis zur Messposition $x_{\text {Regel }}$ : $Z:=\left.A \cdot S_{1}\right|_{\text {mit Keil. }}$ Ist die Rückkopplung nicht vollständig kompensiert, so kann man nun versuchen, die Gegenkopplungsübertragungsfunktion $C$ mit einer zusätzlichen Gegenkopplung 
$\Delta C$ so nachzuadaptieren, dass die Übertragungsfunktion des geregelten Systems

$$
H^{(\mathrm{g})}(C+\Delta C):=\left.\frac{\hat{\eta}\left(x_{\text {Regel }}\right)}{\hat{s}}\right|_{C+\Delta C}
$$

gerade

$$
H^{(\mathrm{g})}(C+\Delta C) \stackrel{!}{=} Z
$$

erreicht, wobei $Z$ eine Zielübertragungsfunktion des Systems mit Gegenkopplung darstellt. Die genaue Wahl von $Z$ wird weiter unten diskutiert und stellt die zu erreichende Minimalkorrelation der stabilisierten Strömung dar. Bei falscher Wahl kann der Regler zur Instabilität neigen.

Betrachtet man das Blockdiagramm in Abb. 5.7, so ist $H^{(\mathrm{g})}(C)$, wenn man die im einzelnen unbekannte physikalische Rückkopplung durch die lineare Übertragungsfunktion $F$ (feedback) im stabilisierten Zustand annähert:

$$
H^{(\mathrm{g})}(C)=\frac{A S_{1}}{1-F S_{1}+A S_{1} M C} .
$$

Soll im $(n+1)$-ten Schritt $H^{(\mathrm{g})}\left(C_{\mathrm{n}+1}\right) \equiv Z$ gelten, so muss die Gegenkopplung durch

$$
\Delta C_{\mathrm{n}}=\frac{1}{M} \cdot\left(\frac{1}{Z}-\frac{1}{H^{(\mathrm{g})}\left(C_{\mathrm{n}}\right)}\right)
$$

korrigiert werden, wobei $M$ die Übertragungsfunktion des Messystems ist (hier eine Verzögerung). Die Messung der aktuellen Übertragungsfunktion $H^{(\mathrm{g})}\left(C_{\mathrm{n}}\right)$ ist im nichtstabilisierten Zustand schwierig, da das auf dem Grenzzyklus schwingende System auf äußere Störung anders reagiert als das stabilisierte System. Bei der Eigenfrequenz des Systems ist daher eine Bestimmung des Übertragungsfaktors nur durch längeres Mitteln möglich. Daher wird für jeden Verbesserungsschritt $n$ mit einem der Schwingungsamplitude angepassten Testsignal die Übertragungsfunktion gemessen und gemittelt. Es ist sinnvoll, sich der Zielfunktion $Z$ langsam anzunähern, indem man den Schrittweitenparameter $0<\mu \leq 1$ einführt. Die Gegenkopplungsübertragungsfunktion wird nun durch

$$
C_{\mathrm{n}+1}=C_{\mathrm{n}}+\mu \Delta C_{\mathrm{n}}
$$

sukzessive berechnet. Durch die Einstellung von $\mu$ steuert man das Folgeverhalten und die Genauigkeit der Adaption.

Um den beschriebenen Algorithmus umzusetzen, muss die jeweils gemessene Übertragungsfunktion $H^{(\mathrm{g})}\left(C_{\mathrm{n}}\right)$ des Systems invertiert werden. Aus dem gemäß Gl. (5.5) resultierenden $\Delta C_{\mathrm{n}}$ wird ein kausales Filter $\Delta c_{\mathrm{n}}$ berechnet und zu dem Gegenkopplungsfilter $c_{\mathrm{n}}$ addiert.

\subsubsection{Messung von $\mathbf{H}^{(\mathrm{g})}(C)$ und Berechnung von $\Delta C$}

Das Testsignal $s(t)$, das hier meistens als kurzer, periodischer eingebrachter Schrödersweep oder als breitbandiges Rauschsignal mit jeweils auf das System angepasstem Leistungsspektrum (Prewhitening) synthetisiert wird, ist im Allgemeinen breitbandiger als die Antwort der 
Streichlinienauslenkung $\hat{\eta}$ am Ort $x_{\text {Regel }}$. Da das Testsignal klein und das dazu unkorrelierte Signal, das hier vor allem durch die selbsterregte Schwingung entsteht, relativ groß ist, wird die Kreuzkorrelation im Spektrum $\left\langle\hat{s}^{*} \hat{\eta}\right\rangle_{t}$ über eine gewisse Zeitdauer gemittelt. Die selbsterregte Schwingung mittelt sich näherungsweise heraus, und man erhält eine im Spektrum und in der Phase glatte Übertragungsfunktion. Es gestaltet sich schwierig, die selbsterregte Schwingung aus den Messdaten durch Prediktion der selbsterregten Schwingung herauszuziehen, um die Mittelungsdauer zu verkürzen. Aufgrund der Nichtlinearität wird die Phase der selbsterregten Schwingung durch das Testsignal beeinflusst. Somit steht jeweils nur ein kurzer Ausschnitt, während der in das Testsignal eingebauten periodisch wiederkehrenden Signalpausen $(s(t)=0)$, zur Prediktion zur Verfügung.

Um das Folgeverhalten des adaptiven Algorithmus zu verbessern, kann der beste Kompromiss zwischen dem Verkürzen der Mittelungszeit und dem Herausmitteln der selbsterregten Schwingung gesucht werden. Als geeignet hat sich eine Gewichtung bei der Mittelung der Kreuzkorrelationen herausgestellt: In jedem Aktualisierungsschritt $n$ der Gegenkopplung wird $\hat{s}$ und $\hat{\eta}$ durch diskrete Kurzzeit-Fouriertransformation von $s(t)$ und $\eta\left(x_{\text {Regel }}, t\right)$ berechnet und die Kreuzkorrelationen der letzten $m$ Schritte $\left(\hat{s}^{*} \hat{\eta}\right)(n) \ldots\left(\hat{s}^{*} \hat{\eta}\right)(n-m)$ gespeichert. Die Mittelung der Kreuzkorrelationen der letzten $m$ Schritte wird durch eine für steigende $k$ abfallende Gewichtsfunktion $g(k): g(k) \neq 0$ für $m>k>0$ entsprechend $\left\langle\left(\hat{s}^{*} \hat{\eta}\right)(k) \cdot g(n-k)\right\rangle_{k}$ durchgeführt. Damit wird die im $n$-ten Schritt bestimmte Kreuzkorrelation $\left(\hat{s}^{*} \hat{\eta}\right)(n)$ bei der Berechnung des Mittels stärker gewichtet. Bezüglich des Folgeverhaltens des Algorithmus stehen der Schrittweitenparameter $\mu$ und die Anzahl $m$ der Mittelungen in einem relativ komplizierten Zusammenhang, da einerseits eine längere Mittelungszeit $m$ ein größeres $\mu$ aufgrund der gestiegenen Genauigkeit der Übertragungsfunktion erlaubt. Andererseits ändert sich mit größerem $\mu$ auch die jeweilige Gegenkopplung schneller, so dass $H^{(\mathrm{g})}\left(C_{\mathrm{n}}\right)$ nicht zu der passenden Gegenkopplung $C_{\mathrm{n}}$ bestimmt wird, sondern über die Systemdynamiken mit den verschiedenen Gegenkopplungen $C_{\mathrm{n} \ldots \mathrm{n}-\mathrm{m}}$ gemittelt wird. Eine gute Wahl von $\mu, m$ und damit ein optimiertes Folgeverhalten des adaptiven Algorithmus kann daher nur empirisch ermittelt werden. Wird $\mu$ zu groß gewählt, führt der adaptive Algorithmus zur Instabilität des Systems, da die vorausgesetzte Quasistationarität nicht mehr gegeben ist, d.h. die Voraussetzung, dass die Dynamik des Gesamtsystems aufgrund der Gegenkopplungsänderung $\mu \Delta c$ sich nur unwesentlich während der zeitlichen Mittelung der Übertragungsfunktion ändert.

Als sehr geeignete Methode zur Bestimmung der Übertragungsfunktion hat sich die TikhonovRegularisierung erwiesen:

$$
H^{(\mathrm{g})}\left(C_{\mathrm{n}}\right)=\cdot \frac{\left\langle\hat{s}^{*} \hat{\eta}\right\rangle_{t}}{\left\langle\hat{s}^{*}\right\rangle_{t}+\epsilon_{\mathrm{Tikh}} .\left\langle\left\langle\hat{s}^{*} \hat{s}\right\rangle_{t}\right\rangle_{f}},
$$

wobei sich hier als beste Wahl des Tikhonov-Parameters $\epsilon_{\text {Tikh. }} \approx 0.01$ bezüglich der mittleren spektralen Leistung des Eingangssignals $\left\langle\left\langle\hat{s}^{*} \hat{s}\right\rangle_{t}\right\rangle_{f}$ herausstellt. Im Gegensatz zu Fensterungen im Frequenzbereich wird hier keine explizit ausgezeichnete Abschneidefrequenz verlangt. Auch 
Kapitel 5. Regelung des Strahl-Kanten-Systems

für die Aktualisierung der Gegenkopplung $\Delta C$ kann die Tikhonov-Regularisierung angewendet werden:

$$
\Delta C=\frac{1}{M} \frac{Z^{*}\left|H^{(\mathrm{g})}\left(C_{\mathrm{n}}\right)\right|^{2}-H^{(\mathrm{g})}\left(C_{\mathrm{n}}\right)^{*}|Z|^{2}}{\left|Z H^{(\mathrm{g})}\left(C_{\mathrm{n}}\right)\right|^{2}+\epsilon_{\text {Tikh. }}\left\langle\left|Z H^{(\mathrm{g})}\left(C_{\mathrm{n}}\right)\right|^{2}\right\rangle_{f}} .
$$

Eines der wesentlichen Probleme ist die nicht gegebene Kausalität von $\Delta C$ gemäß (Gl. (5.5)). Nur der kausale Anteil $\left(\left.\Delta c\right|_{t \geq 0}\right)$ von $\Delta C$ wird in das Gegenkopplungsfilter bzw. in die Impulsantwort $c$ der Gegenkopplung

$$
\Delta c=\mathcal{F}^{-1}\left\{[\Delta C]_{\text {kausal }}\right\}=\left.\mathcal{F}^{-1}\{\Delta C\}\right|_{t \geq 0}
$$

integriert. Es hat sich zusätzlich als nützlich erwiesen, $\Delta c$ mit einem Fenster im Zeitbereich zu gewichten, so dass die Impulsantwort für größere $t$ stärker abfällt. Wenn die Impulsantwort für diese Zeiten zur Erreichung der Zieldynamik tatsächlich notwendig ist und somit nicht aus Mess- bzw. Schätzfehlern resultiert, baut der adaptive Algorithmus diese später auf. Auch im Frequenzbereich wird $\Delta C$ prinzipiell durch die Tikhonov-Methode Gl. (5.8) gefiltert, jedoch

ohne feste Abschneidefrequenz. Da $[\Delta C]_{\text {kausal }}$ höhere Frequenzkomponenten als $\Delta C$ besitzen kann, wird die Gegenkopplung $C$ zusätzlich bandbegrenzt.

\subsubsection{Stabilität des adaptiven Algorithmus und Wahl der Zielfunk- tion}

Die Wahl einer sinnvollen Zielfunktion $Z$ bestimmt sehr wesentlich die Stabiliät des Systems, da die Gegenkopplung durch $\Delta C=M^{-1}\left(Z^{-1}-\left(H^{(\mathrm{g})}(C)\right)^{-1}\right)$ mit der Zielfunktion verbunden ist. Wünschenswert für die Stabilisierung des Strahl-Kanten-Systems ist eine Zielfunktion, die zu einem kleinem Leistungstransfer und einer zeitlich kurzen Impulsantwort von $\mathcal{F}^{-1}\left\{H^{(\mathrm{g})}(C)\right\}$ führt.

Die Anwesenheit des Keils erzeugt eine zusätzliche Korrelation zwischen dem Eingangssignal $s(t)$ und dem Messsignal $\eta\left(x_{\text {Regel }}, t\right)$. Benutzt man als Zielfunktion die vom Gesamtsystem minimal realisierbare Korrelation, so kompensiert die Gegenkopplung zwangsläufig die zusätzliche Korrelation durch die Strahl-Kanten-Wechselwirkung. Fordert man jedoch eine kleinere realisierbare Korrelation als Zielfunktion, so kann die Gegenkopplung die gewünschte Übertragungsfunktion nicht einstellen, und das geregelte System kann instabil werden.

Betrachtet sei zunächst die minimale, realisierbare Korrelation der konvektiven Instabilität des Strahls in Abbildung 5.8. Gesucht ist eine Gegenkopplung, die das Messsignal $\eta(t)$ an der Stelle $x_{\text {Regel }}$ und das Eingangssignal mit Gegenkopplung $s(t)-(c * \eta)(t)$ möglichst gut für positive Zeitdifferenzen dekorreliert:

$$
\langle\eta \cdot[s(t-\Delta t)-(c * \eta)(t-\Delta t)]\rangle_{\Delta t>0}=0 .
$$

Da $\hat{s} / \hat{\eta}=(1+H C) / H$, ist die größte Dekorrelation gegeben, wenn der kausale Anteil von 


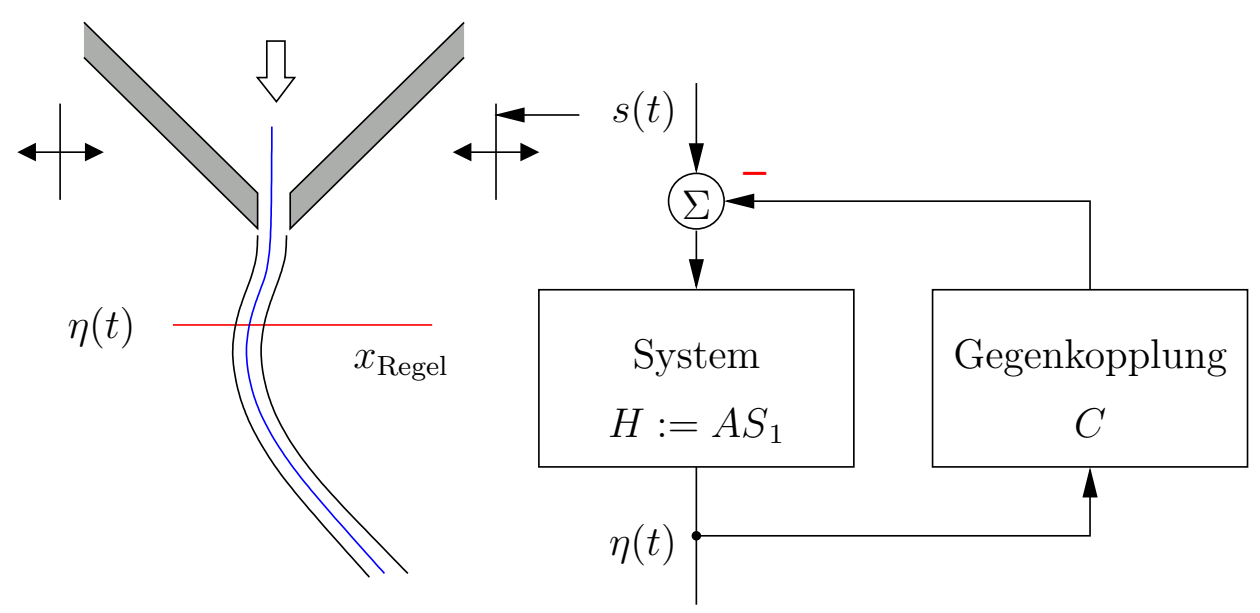

Abb. 5.8: Minimierung der Korrelation der konvektiven Instabiliät. Blockschaltbild der Gegenkopplung.

$[\hat{s} / \hat{\eta}]_{\text {kausal }}=0$ und somit $C=-\left[H^{-1}\right]_{\text {kausal }}$ ist. Verwendet man daher als Gegenkopplungsverbesserung den kausalen Anteil der Übertragungsfunktion des geschlossenen Kreises

$$
\Delta c=-\mu \cdot \mathcal{F}^{-1}\left\{\left[H^{(g)^{-1}}\right]_{\text {kausal }}\right\}
$$

so kann $[\hat{s} / \hat{\eta}]_{\text {kausal }}$ deutlich verringert werden (Abb. 5.9), und der akausale Anteil steigt leicht an. Dabei kann der kausale Anteil an einer weiter stromauf liegenden Regelposition (Abb. 5.9 (Mitte)) besser unterdrückt werden. Allerdings neigt der adaptive Algorithmus für längere Laufzeiten bzw. große Gegenkopplungen zur Instabilität. Daher ist es sinnvoll, einen gewissen kausalen Anteil von $H^{(g)^{-1}}$ in $H^{(g)}$ zuzulassen, der mit der Zielfunktion $Z$ schon bezeichnet ist. Diese minimale zugelassene Übertragungsfunktion $Z$ kann nur a priori durch Modellannahmen geschätzt werden. Die Dekorrelation durch die Gegenkopplung kann für das eben betrachtete Beispiel die durch die Physik fest vorgegebene Ausbreitung der konvektiven Instabiliät nur zu einem geringen Anteil kompensieren, so dass es sinnvoll ist, diese als Zielfunktion zu benutzen. Kommt jedoch eine zusätzliche Korrelation durch die physikalische Rückkopplung des Keils hinzu, so ist der adaptive Algorithmus durch Einstellen der Gegenkopplung in der Lage, die zusätzliche Korrelation zu kompensieren. Dabei wird $H^{(g)}$ durch die Adaption des Gegenkopplungsfilters allmählich auf die Wunschübertragungsfunktion $Z$ gebracht. Damit kommt auch die selbsterregte Schwingung zum Erliegen und der Fixpunkt der Strahl-Kanten-Dynamik wird stabilisiert.

Im Folgenden wird als Zielübertragungsfunktion

$$
Z=\left.K_{\mathrm{L}} \cdot\left(A \cdot S_{1}\right)\right|_{\text {ohne Keil }}
$$

die Übertragungsfunktion der konvektiven Instabilität bis zum Messort $x_{\text {Regel }}$ verwendet. Der Faktor $K_{\mathrm{L}}$ beschreibt die größere Effektivität des Aktuators bei kleineren Keilabständen $L$, da 


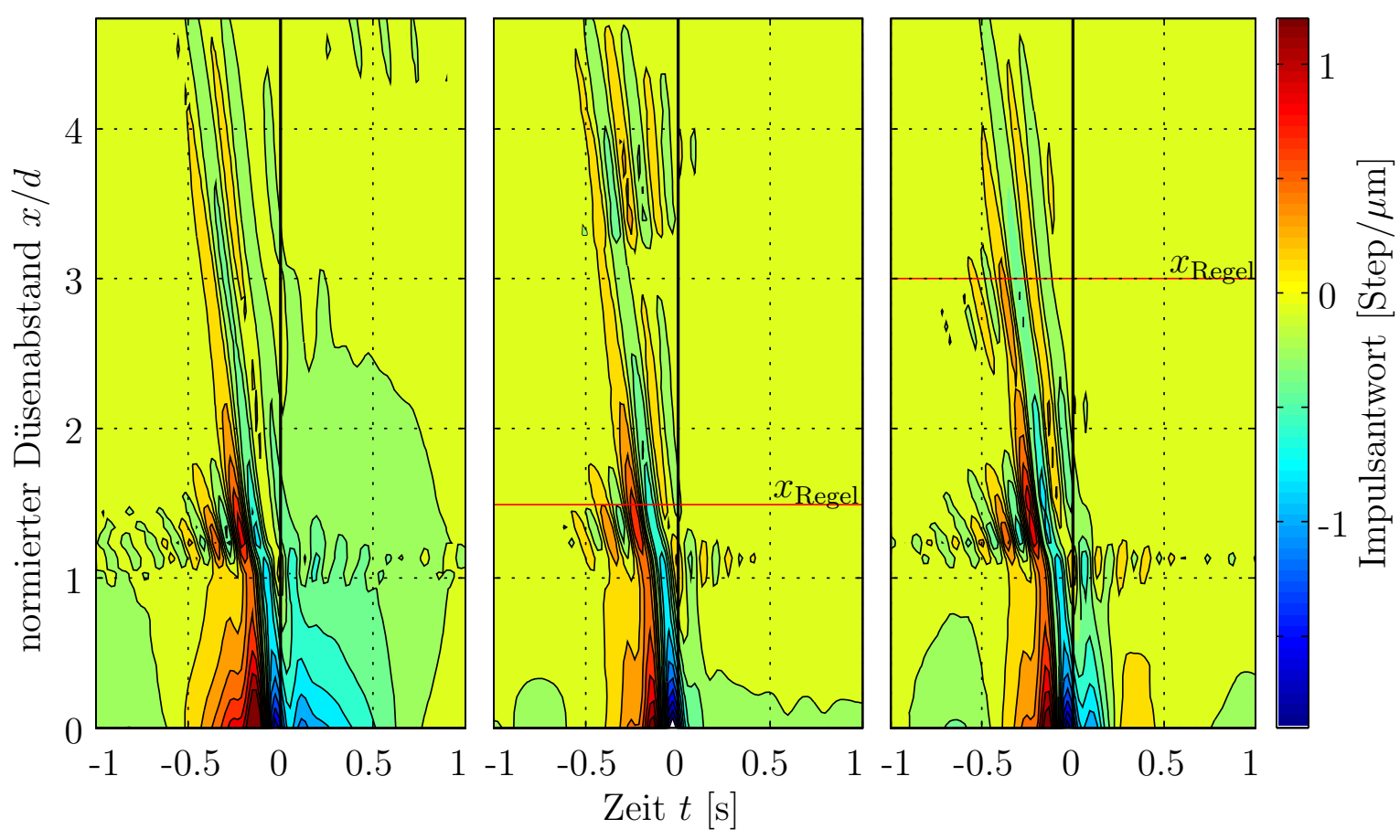

Abb. 5.9: Impulsantwort der inversen Übertragungsfunktion $\boldsymbol{H}^{(\boldsymbol{g})^{-\mathbf{1}}}=\hat{\boldsymbol{s}} / \hat{\boldsymbol{\eta}}$ farbkodiert in $[$ Steps $/ \mu \mathrm{m}]$. Links: ohne Gegenkopplung, Mitte: mit Gegenkopplung von $x_{\text {Regel }}=1.5 d$, unten mit Gegenkopplung von $x_{\text {Regel }}=3 d$.

bei verschiedenen Keilabständen $L$ das durch den Aktuator verschobene Flüssigkeitsvolumen zu unterschiedlichen lateralen Auslenkungen $|\hat{\zeta}|$ unterhalb der Düse führt. Dieser Faktor $K_{\mathrm{L}}$ kann aus der Potenzialtheorie ermittelt werden (Schwarz-Christoffel-Transformation). Damit wird im Folgenden davon ausgegangen, dass

$$
\left.\left.\left(A \cdot S_{1}\right)\right|_{\text {mit Keil }} \approx K_{\mathrm{L}} \cdot\left(A \cdot S_{1}\right)\right|_{\text {ohne Keil }}
$$

ist. Die Stabilität des Systems, die durch ein breitbandiges Rauschsignal gemessen wird, zeigt je nach Wahl von $K_{\mathrm{L}}$ unterschiedliche Güte. Benutzt man das aus der Potenzialtheorie kommende $K_{\mathrm{L}}$, so ist die Stabilität in der Tat am größten, und die spektrale Leistungsdichte zeigt keine ausgeprägten Spitzen.

In Abb. 5.10 ist die Zeitreihe $\eta\left(x_{\text {Mess. }}\right)$ während eines typischen Stabilisierungsvorgangs für $L / d=6$ gezeigt. Die Amplitude des Sweeps $s(t)$ wird durch die kurzzeitgemittelte spektrale Leistungsdichte automatisch angepasst, um immer ein optimales Signal/Rausch-Verhältnis zu gewährleisten. Die spektrale Leistungsdichte wird daher mit einem zeitlich linear abnehmenden Vergessensfaktor gewichtet. Die Anfangsamplitude des Sweeps wird hier mit $\left|s^{\max }\right|_{\text {Start }}=400$ Steps festgelegt und mit abnehmender selbsterregter Schwingungsamplitude auf $\left|s^{\max }\right|_{\text {Ende }}=100$ Steps geregelt. Nach circa 100 Perioden der selbsterregten Schwingung 

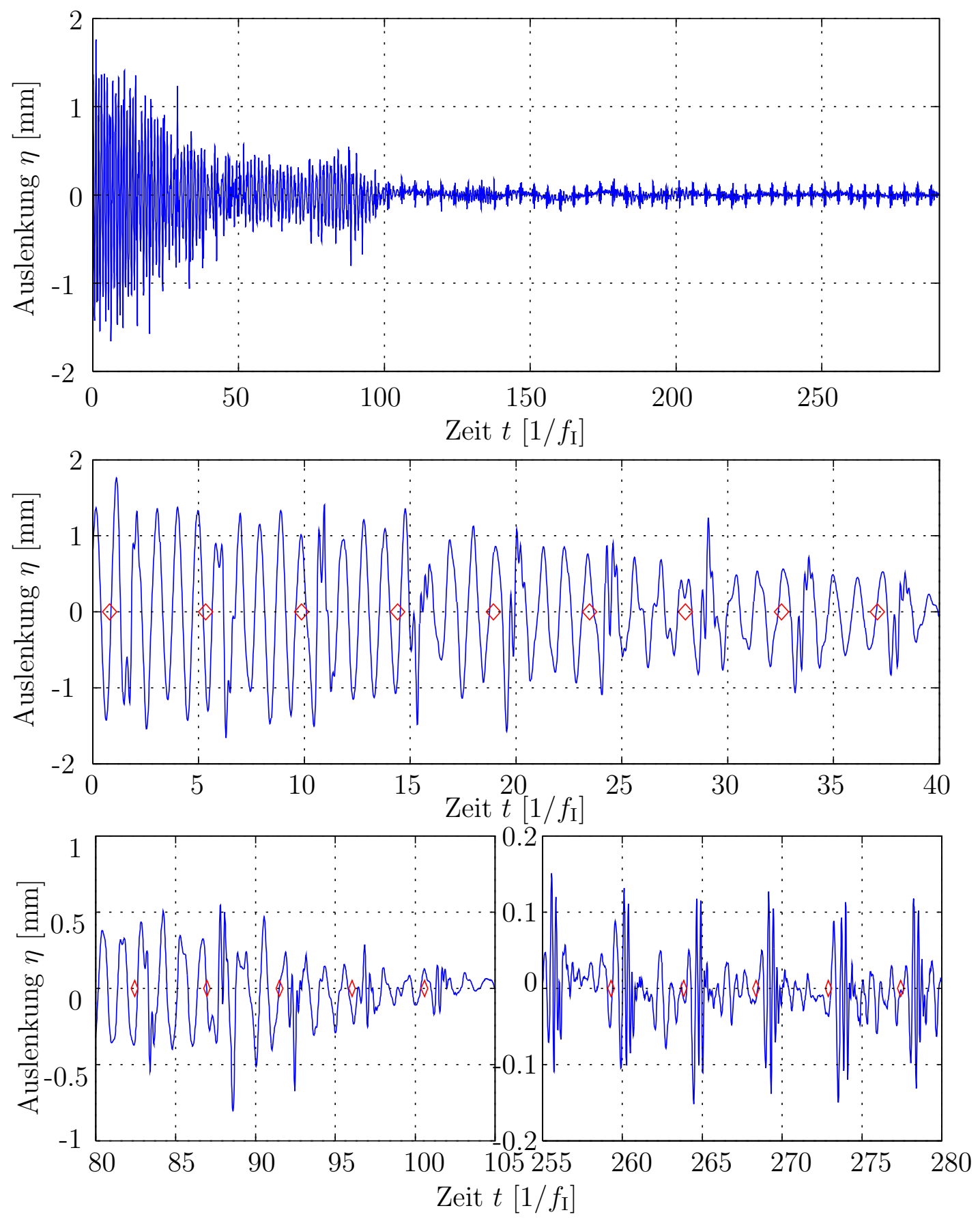

Abb. 5.10: Zeitreihe der Streichlinienauslenkung während einer Stabilisierung. Das periodisch wiederholte Testsignal besteht aus einem Sweep der Länge 200/ $f_{\text {sampling }}$ (4 Sekunden) und anschließend $56 / f_{\text {sampling }}$ Pause $(s(t)=0)$. Die Periodizität ist in der Zeitreihe durch rote Rauten markiert. Parameter: Eigenfrequenz $f_{\mathrm{I}}=0.88 \mathrm{~Hz}, U_{\mathrm{D}}=5.36 \mathrm{~cm} / \mathrm{s}, \operatorname{Re}_{\mathrm{d}}=235$, $T=24^{\circ} \mathrm{C}, L / d=6, d=4 \mathrm{~mm}, x_{\text {Mess. }} / d=3.5, x_{\text {Regel }} / d=0.5$. 

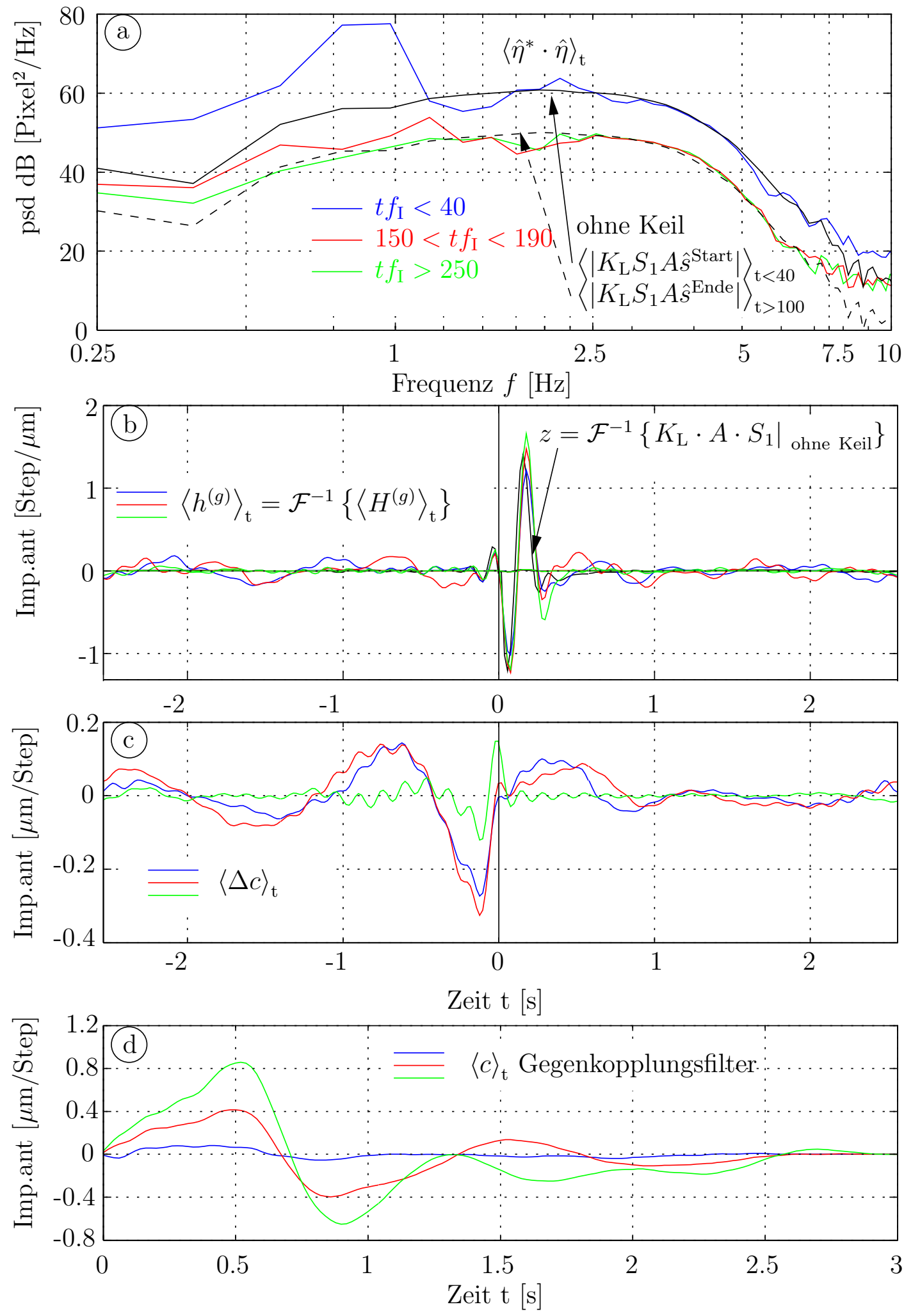

Abb. 5.11: Verschiedene Stadien einer Stabilisierung. Parameter wie in Abb. 5.10. 
$\left(t f_{\mathrm{I}}>100\right)$ ist eine Stabilisierung annähernd erreicht. In Abb. 5.11 (a) sind die spektralen Leistungsdichten bei verschiedenen Stabilisierungsgraden mit den zugehörigen Impulsantworten von $\Delta C$ im Vergleich zu $Z$ gezeigt. Für $150<t f_{\mathrm{I}}<190$ stimmen die Leistungsspektren etwa überein, obwohl sich die Impulsantwort der Zielfunktion $z$ noch wesentlich von der aktuell gemessenen Impulsantwort $H^{(g)}$ (vgl. Abb. 5.11 (b) z (schwarz) mit $H^{(g)}$ (rot)) unterscheidet. Dabei ist vor allem die zeitliche Ausdehnung der Impulsantwort wesentlich, da die verzögerten Anteile die noch nicht kompensierte Strahl-Kanten-Wechselwirkung darstellen. Verglichen mit dem

anfänglichen $\langle\Delta c\rangle_{0<t f_{\mathrm{I}}<40}$ (blaue Kurven) ist der gemessene kausale Anteil von $\langle\Delta c\rangle_{150<t f_{\mathrm{I}}<190}$ durch die Regelung nicht wesentlich kleiner geworden.

Mit zunehmender Adaption sinkt dann der kausale Anteil von $\Delta c$, und das Leistungsspektrum von $H^{(g)}$ nähert das geschätzte erreichbare ohne Keil sehr gut an. Die Gegenkopplung ist für $f_{\mathrm{I}}>250$ auskonvergiert. Der noch verbleibende kausale Anteil von $\Delta c$ in Abb. 5.11 (grüne Kurve) wird nicht in das Gegenkopplungsfilter übernommen, da in der Implementierung des adaptiven Algorithmus zur Erniedrigung der Fehleranfälligkeit zusätzlich die Bandbreite von $C$ begrenzt wird. Die auskonvergierte Gegenkopplung bewirkt, dass die spektralen Leistungsdichten des geregelten Systems und des Systems ohne Keil (aber mit Keilkorrektur $K_{\mathrm{L}}$ ) sehr gut übereinstimmen.

Ohne die Hystereseinvertierung aus Kap. 2.4 wächst die Gegenkopplung bei immer kleineren Anregungsamplituden $s(t)$ aufgrund der betragsmäßig unterschätzen Übertragungsfunktion $H^{(g)}$ immer weiter an, so dass die Gegenkopplung nicht konvergiert und das Gesamtsystem damit instabil werden kann.

\subsection{Parameter und Grenze der adaptiven Regelung}

Bevor eine Grenze der adaptiven Regelung definiert wird, werden in den nächsten Unterabschnitten ausgewählte Parameterabhängigkeiten untersucht. Für den adaptiven Algorithmus müssen einige freie Parameter festgelegt werden, von deren Wahl die Grenze und die Güte der Stabilisierung wesentlich abhängen:

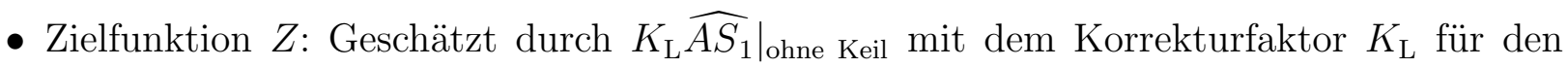
Aktuator aus der Potenzialtheorie.

- Regelposition $x_{\text {Regel }}$ : Düsennähe bringt höhere Stabilität während der Stabilisierungsphase des im Grenzzyklus schwingenden Systems. Mehrere Regelpositionen bringen qualitativ kaum Verbesserungen (Kap. 5.4.2).

- Länge des Gegenkopplungsfilter: einige Schwingungsperioden (Kap. 5.5).

- Testsignalamplitude und Testsignalform: Amplitude groß, Testsignal breitbandig und auf Schwingungsfrequenz abgestimmt. 
- Schrittweitenparameter: kleiner für größere $L / d$.

- Mittelungsdauer, die zur sicheren Bestimmung der Übertragungsfunktionen notwendig ist: lang gegen Schwingungsdauer.

\subsubsection{Wahl der optimalen Regelposition}

Die Wahl der Regelposition $x_{\text {Regel }}$ beeinflusst sowohl die Güte des stabilisierten Zustands als auch die Stabilität der Adaption des Gegenkopplungsfilters während der Unterdrückung der selbsterregten Schwingung.

Dabei wird die Güte als Differenz zwischen mittlerer Leistung des ungeregelten Systems und mittlerer Leistung des mit der Amplitude 100 Steps angeregten Systems definiert.

Die Stabilität der Adaption wird durch die maximale Geschwindigkeit gemessen, mit der die selbsterregte Schwingung unterdrückt wird: Mit wachsendem Schrittweitenparameter $\mu$ wird die Stabilisierung der Strömung immer schneller erreicht, jedoch wächst bei fester Anfangstestsignalamplitude $\left|s^{\max }\right|_{\text {Start }}$ die Wahrscheinlichkeit, dass eine Gegenkopplung adaptiert wird, so dass das schon instabile System während der Adaptionsphase noch instabiler wird. D.h., die instabilen Pole des geschlossenen Systems wandern nicht in Richtung stabiles Gebiet. Daher wird die Stabilität der Adaption definiert als der Anteil der zur Stabilisierung führenden Adaptionen bei fest

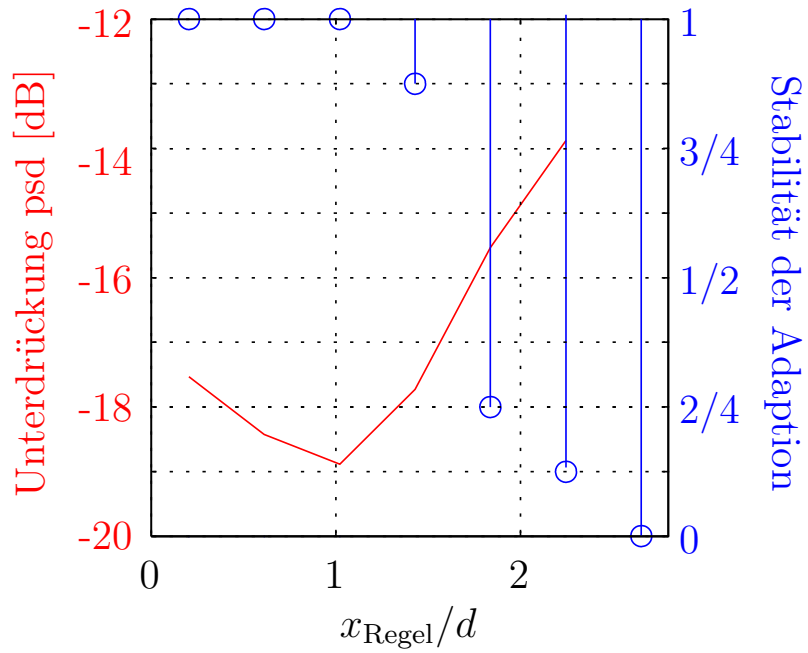

Abb. 5.12: Güte der Stabilisierung und Stabiltät der Adaption während der Stabilisierungsphase für $\mu=0.5,\left|s^{\max }\right|_{\text {Start }}=400$ Steps. vorgegebenem $\mu$ und fest vorgegebener Anfangsamplitude $\left|s^{\max }\right|_{\text {Start }}$ des Testsignals. Die Amplitude des Testisgnals $\left|s^{\max }\right|_{\text {Start }}$ ist mitentscheidend für den Informationsgewinn pro Adaptionsschritt.

In Abb. 5.12 ist der Anteil der zur Stabilisierung führenden Messungen gegen die Regelposition aufgetragen. Zu Beginn der Messung ist die Testsignalamplitude $\left|s^{\max }\right|_{\text {Start }}=400$ Steps gewählt. An sieben verschiedene Regelpositionen $x_{\text {Regel }}$ mit jeweils acht Messungen von circa 300 Schwingungsdauern Länge wird die Stabilität der Adaption und die Güte des stabilisierten Zustands getestet. Der Schrittweitenparameter wird dabei konstant $\mu=0.5$ gewählt (üblich sind sonst $0.05<\mu<0.3)$. Alle Adaptionen, die das System in der vorgegebenen Zeit nicht vom Grenz- 
zyklus zum Fixpunkt gebracht haben, werden als instabil bezüglich der Adaption gewertet. Da $\mu$ relativ groß gewählt ist, zeigt sich häufig nach relativ kurzer Zeit $\left(100 / f^{\mathrm{T}}\right)$, ob die Adaption zur Stabilisierung führt oder nicht, so dass die Grenze zwischen den stabilisierenden und den nicht stabilisierenden Adaptionen sehr scharf ist.

Die düsennahen Regelpositionen $x_{\text {Regel }}$ zeichnen sich durch besonders hohe Stabilität bezüglich der Adaption aus. Die für den Adaptionsprozess notwendigen Informationen scheinen dort in besonderer Klarheit auswertbar. Insbesondere kann die Rückkopplung des Keils in Düsennähe sehr direkt gemessen werden, da die Streichlinienauslenkung vom Anregungsfeld der StrahlKanten-Wechselwirkung dominiert ist. Daher kann die Information über die Keilrückkopplung dort besonders gut extrahiert werden.

Das Amplitudenverhältnis zwischen der dritten und der ersten Harmonischen ist für verschiedene $L / d$ im Düsennahbereich erhöht (Abb. 5.13), was darauf schließen lässt, dass sich dort der am Keil stattfindende Mechanismus stark widerspiegelt. Die konvektive Instabilität hat bei ähnlicher Auslenkungsamplitude ein wesentlich geringeres Verhältnis der Harmonischen. Weiter stromab wächst dann die Nichtlinearität an und führt zu schwierigen Vermischungen einzelner Spektralkomponenten (siehe Kap. 3.5.2).

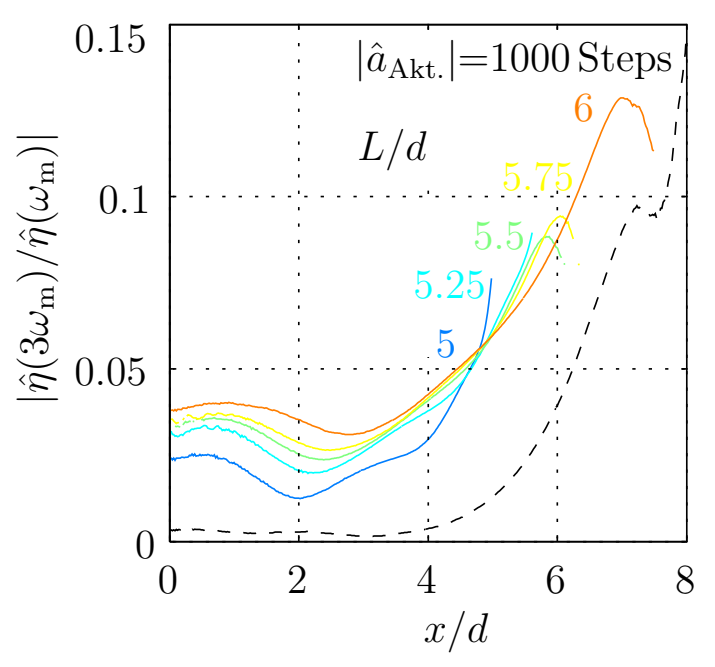

Andererseits ist die Messgenauigkeit unterhalb der Düse auf Grund der sehr kleinen Auslenkungen von wenigen $\mu \mathrm{m}$ am geringsten, so dass schon kleine MessungeAbb. 5.13: Nichtlinearität der Streichlinie für Mode I. $U_{\mathrm{D}}=5.36 \mathrm{~cm} / \mathrm{s}$, schwarz: konvektive Instabilität $1 \mathrm{~Hz}$.

nauigkeiten bei der Interpolation zwischen den Bildelementen der CCD-Kamera (siehe Kap. 2.3) zu einer verminderten Qualität der Stabilisierung führen.

Die beste Unterdrückung der Instabilität kann mit einer Regelposition erreicht werden, die circa eine Düsenbreite stromab liegt. Für größere $L / d$ wird der Bereich der bezüglich der Adaption stabilen Reglerpositionen $x_{\text {Regel }}$ immer kleiner, so dass Positionen in Düsennähe gewählt werden müssen, für die die erreichte Güte aufgrund der Stabilisierung nicht optimal ist (siehe Abb.5.12).

\subsubsection{Parallel laufende adaptive Algorithmen}

Zur Erhöhung der Messgenauigkeit bietet es sich an, von $p$ Messpositionen aus zu regeln, daher wird der vorgestellte adaptive Algorithmus parallel angewendet, wie in Abb. 5.14 dargestellt. 


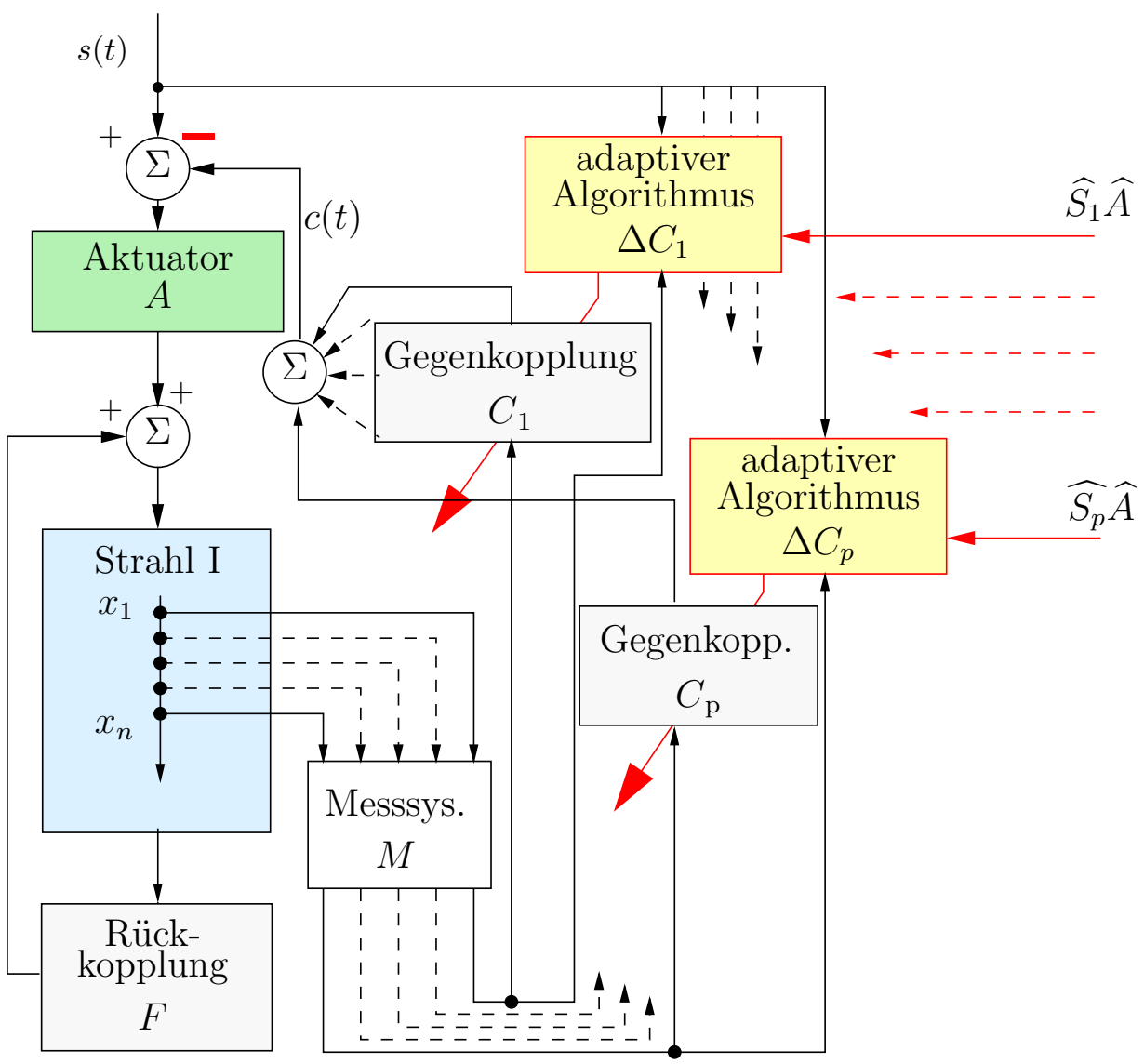

Abb. 5.14: Blockschaltbild mehrerer paralleler adaptiver Algorithmen. Die gestrichelten Linien deuten die Filter 2 bis p-1 an.

Die Schrittweitenparameter $\mu_{j}, 1 \leq j \leq p$ müssen entsprechend der Anzahl $p$ der parallelen Filter reduziert werden. Vorteilhaft ist eine ansteigende Gewichtung der $\mu$ mit kleiner werdendem Düsenabstand der Messposition, da damit die größte Stabilität während der Unterdrückung der Schwingung gegeben ist.

In Abb. 5.15 ist für gleiche Parameter ein Vergleich der adaptiven Stabilisierungen mit fünf parallel laufenden Filtern bzw. mit einem Filter gezeigt. Der Unterschied liegt bei abgeschaltetem Testsignal bei etwa $15 \mathrm{~dB}$. Jedoch ist es nicht möglich, die Grenze der Regelbarkeit in der $\operatorname{Re}_{\mathrm{d}}-L / d$-Ebene mittels der größeren erreichten Messgenauigkeit wesentlich zu erhöhen.

\subsubsection{Grenze der Regelung in der $R_{\mathrm{d}}-\mathrm{L} / \mathrm{d}-\mathrm{Ebene}$.}

Die Grenze der Regelbarkeit durch den hier verwendeten adaptiven Algoritmus ist in Abb. 5.17 eingezeichnet. Die Reynoldszahl $\mathrm{Re}_{\mathrm{d}}$ wird dabei für die Temperatur des Strahls berechnet, so dass die Temperaturdifferenz zwischen Strahl und umgebendem Medium dabei nur indirekt 


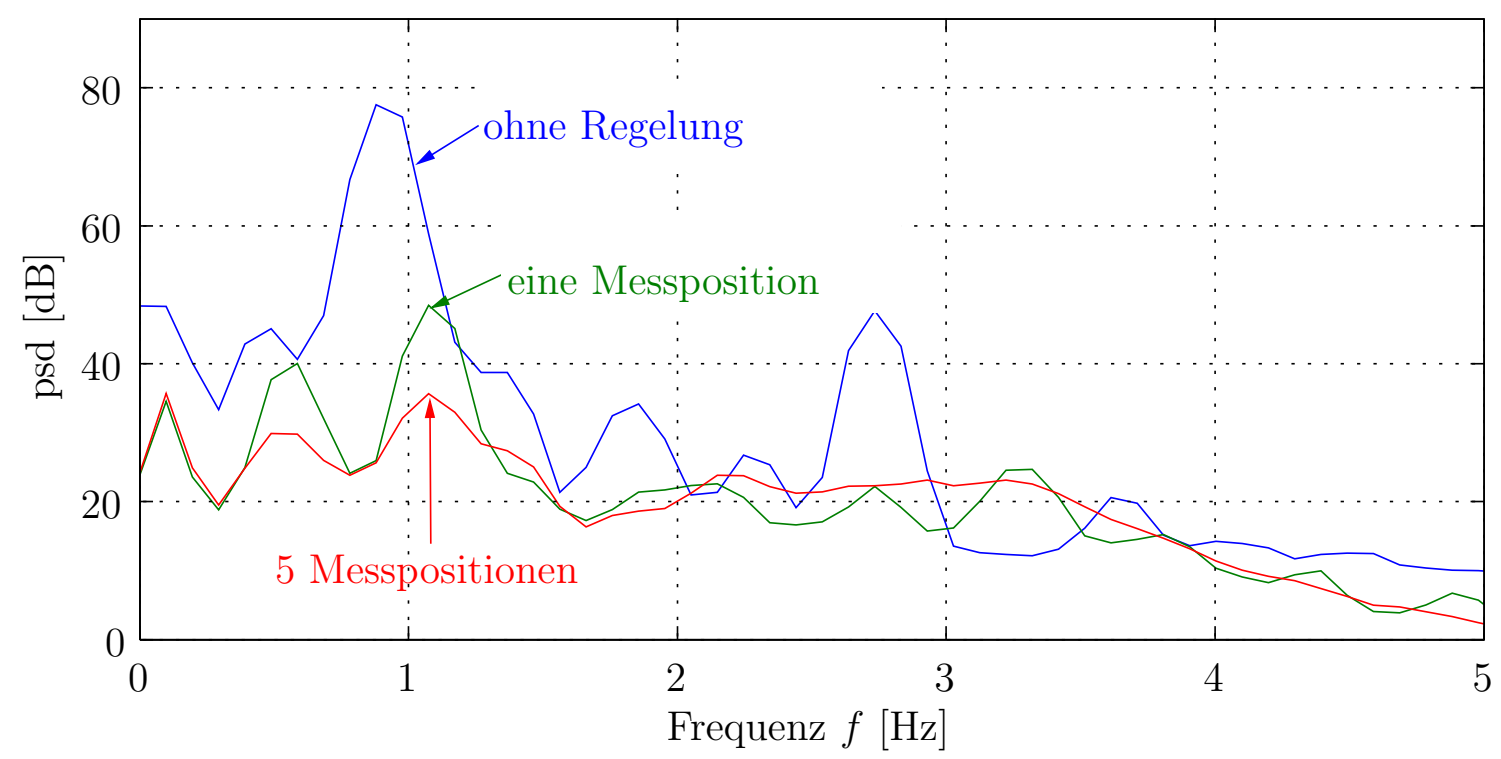

Abb. 5.15: Unterdrückung der selbsterregten Schwingung durch parallele adaptive Algorithmen. $L / d=7.5$, spektrale Leistungsdichte ohne Testsignal, $\mu=0.3, x_{\text {Regel }} / d \approx 1.0$ bzw. $\underline{\mu}=[0.5,0.4,0.3,0.2,0.1] / 5, \underline{x}_{\text {Regel }} / d \approx[0.2,0.6,1.0,1.4,1.8]$.

eingeht. Der Auftrieb des Strahls gegenüber dem Medium innerhalb des Kanals sorgt allem Anschein nach noch für eine zusätzliche destabilisierende Wirkung (Kap. 3.3), die nicht in der Reynoldszahl enthalten ist. Zur Beschreibung dieser Abhängigkeit reicht die Datenmenge der Kompensationen bei verschiedenen Temperaturen aber nicht aus, so dass hier die Grenze in der $\operatorname{Re}_{\mathrm{d}}-L / d$-Ebene dargestellt wird.

Im Folgenden soll festgelegt werden, was unter der Grenze der Stabilisierbarkeit verstanden werden soll.

Unterhalb der Grenze sind Unterdrückungen der selbsterregten Schwingung bis zur Auflösungsgrenze des Bildverarbeitungssystems möglich (> $50 \mathrm{~dB}$ abhängig von der Messposition). Hat das geregelte System den Grenzzyklus verlassen, so kann mit kleinem Schrittweitenparameter $\mu$ die Gegenkopplung verbessert werden, so dass sich das System vollständig beruhigt und zunächst vorhandene Störungen verschwinden bei Andauern des stabilisierten Zustands.

Nahe der Grenze der Stabilisierbarkeit führt die Adaption zu zwei verschiedenen Fällen:

(a) Die natürliche Mode kann fast unterdrückt werden. Jedoch führt das System noch Schwingungen kleiner Auslenkung auf verschiedenen, sich meist abwechselnden Frequenzen aus, die auch durch Nachadaption nicht stabilisiert werden können. Das geregelte System hat also mehrere, sich zeitlich abwechselnde existenzfähige Zustände.

(b) Schon die Adaption schlägt fehl und das System wird auf einer durch den Regler induzierten Mode instabil (hier meistens eine Subharmonische der natürlichen Frequenz). 


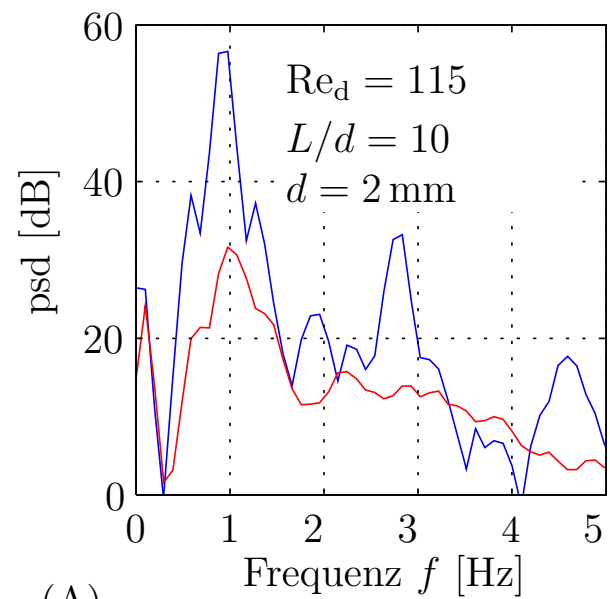

(A)

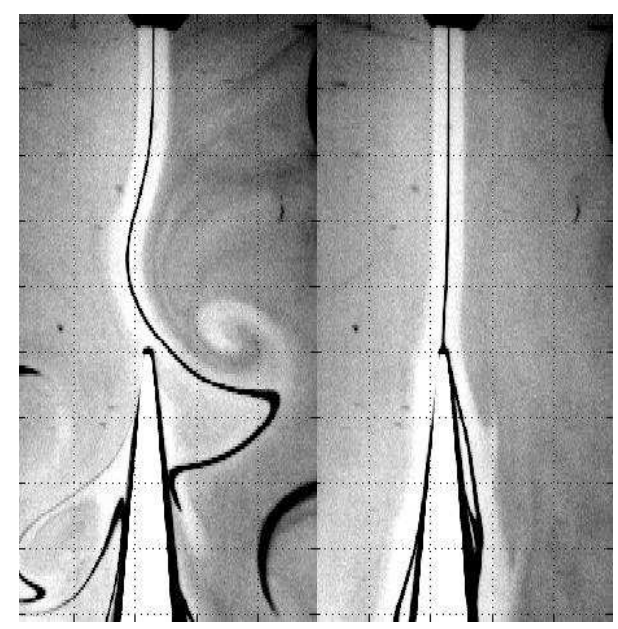

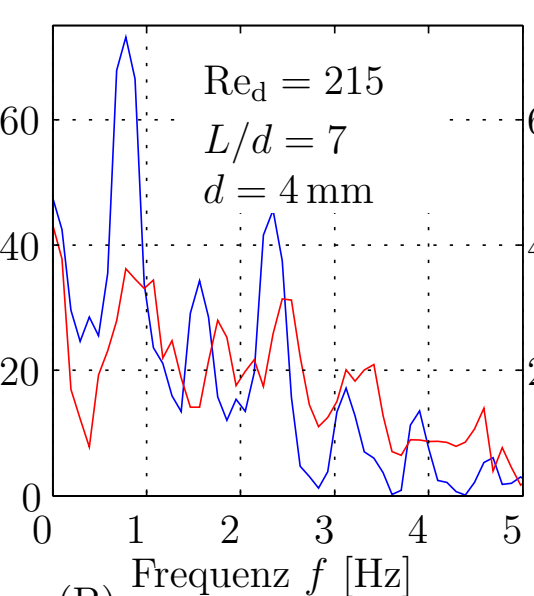

(B)
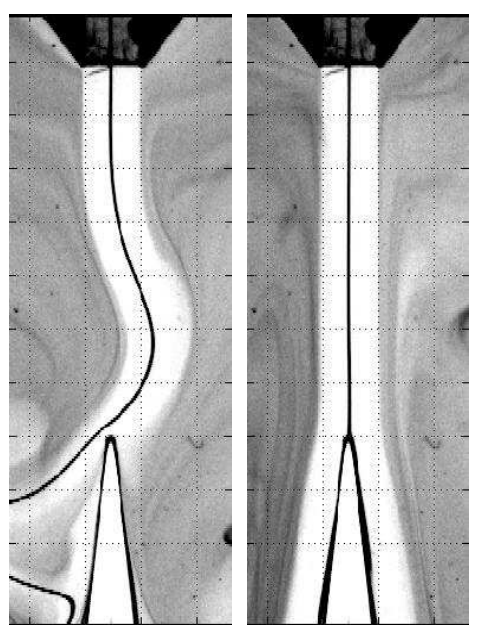

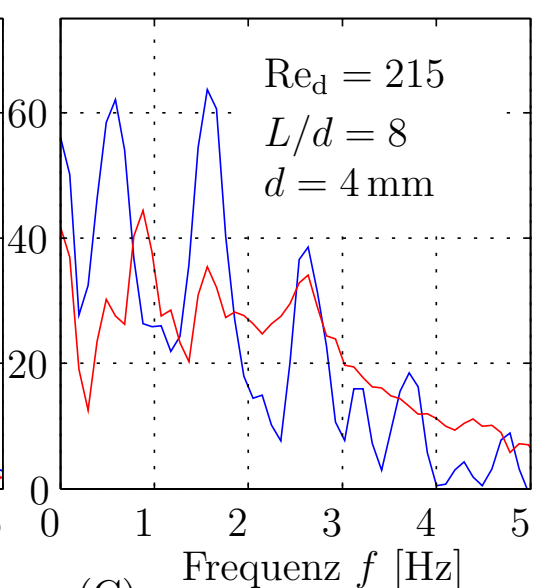

(C)
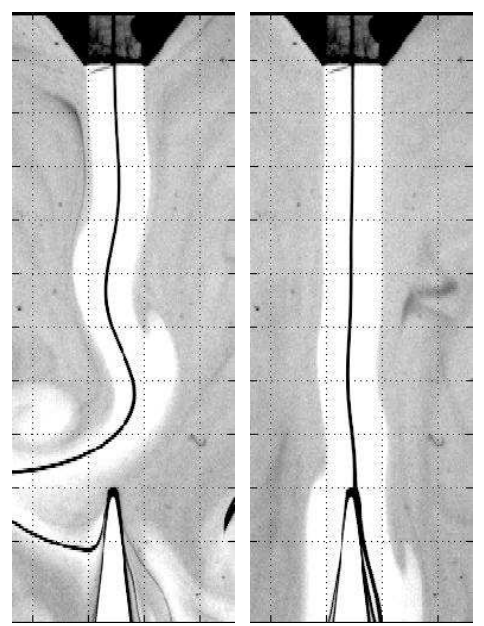

Abb. 5.16: Leistungsspektren und Bilder der Kompensation für verschiedene $L / d$ und $\mathrm{Re}_{\mathrm{d}}$. Ungeregeltes System: blaue Linien und linke Bilder, geregeltes System: rote Linien und rechte Bilder. Gitterabstand $4 \mathrm{~mm}$. 


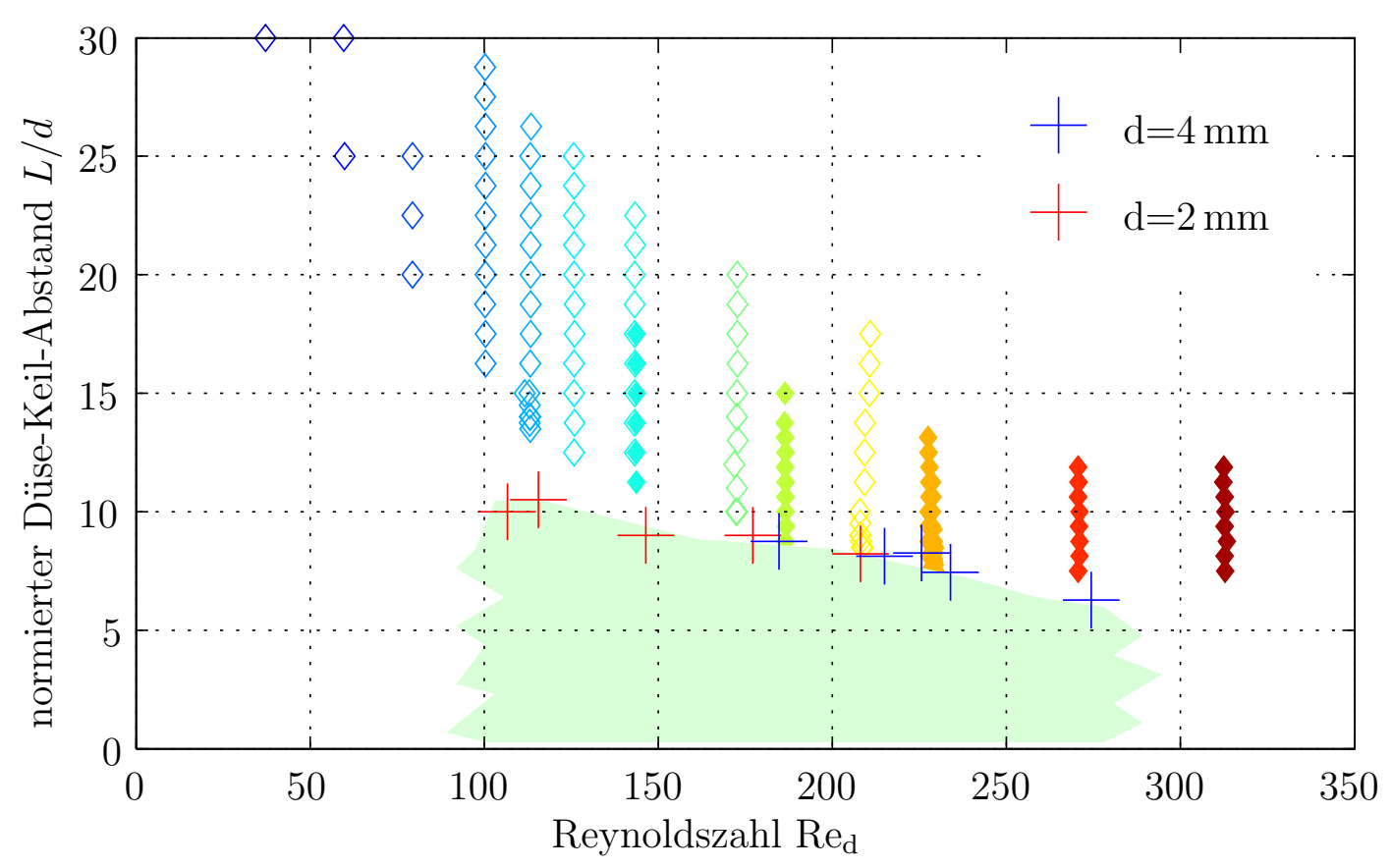

Abb. 5.17: Grenze der Regelung in der $\operatorname{Re}_{\mathrm{d}}-L / d$-Ebene im Vergleich mit dem Existenzgebiet des Systemzustands II. Kreuze kennzeichnen die Grenze des hier untersuchten stabilisierbaren Bereichs $L / d$ (grün). Rauten kennzeichnen die Düse-Keil-Abstände, an denen die Mode II gemessen wurde. Offene Rauten: $\mathrm{d}=2 \mathrm{~mm}$, gefüllte Rauten $\mathrm{d}=4 \mathrm{~mm}$.

Die Grenze der Stabilisierung wird durch eine Unterdrückung von $20 \mathrm{~dB}$ festgelegt, so dass die in (a) erwähnten kleinen Schwingungen bis zu einem gewissen Grad noch erlaubt sind. Die Trennschärfe der Definition ist sehr hoch, da Fall (a) und Fall (b) gekoppelt auftreten, wenn der kritische Keilabstand $L_{\text {krit. }}$ erreicht ist. Eine Unterdrückung der natürlichen Mode gelingt dann trotz ausgefeilterer Parameter selten und nur mit relativ großen Restschwankungen. Die erreichbare Unterdrückung nimmt in der Nähe von $L_{\text {krit. }}$ stark ab.

In Abb. 5.16 (B) ist für $\operatorname{Re}_{\mathrm{d}}=215$ bei $L / d=7$ eine Unterdrückung von etwa $40 \mathrm{~dB}$ möglich und das Video-Bild (B) des stabilisierten Zustands ist im wesentlichen störungsfrei. Bei den Stabilisierungen an der Grenze ((A) für $L / d=10, \operatorname{Re}_{\mathrm{d}}=115$ und (C) für $L / d=8, \operatorname{Re}_{\mathrm{d}}=215$ ) treten diese Störungen vor allem in Keilnähe auf (siehe zugehörige Video-Bilder). Die erreichte Unterdrückung ist deutlich geringer (Leistungsspektren in Abb. 5.16 (A) v.a. (C)). An der Grenze der Kompensation treten Schwingungen kleiner Amplitude auf, deren Schwingungsform ähnlich den entsprechenden natürlichen Moden ist (Video-Bilder A, C). Die entstehenden Leistungsspektren im kompensierten Zustand zeigen in (A) bei der natürlichen Schwingungsfrequenz ein Maximum und in (C) eine Spitze zwischen den natürlichen Moden des Systemzustands II. Die Grenze der Stabilisierbarkeit in Abb. 5.17 orientiert sich an dem unteren Rand des Existenz- 
gebietes des Systemzustands II. Inwieweit jedoch die Dynamik des in Kap. 4.7.1 dargestellten Systemzustands II für die Regelbarkeit entscheidend ist, kann nicht direkt nachgewiesen werden. Die in Abb. 5.6 gezeigten periodisch wiederkehrenden Oszillationen (Fall (a)) tauchen ebenso an der Grenze der Stabilisierbarkeit mittels des adaptiven Algorithmus auf, und die Zeitskalen sind ähnlich. Einerseits kann der adaptive Algorithmus dieser tieffrequenten Dynamik offensichtlich nur unzureichend folgen. Andererseits kann er durch Verringerung der Mittelungszeit und Erhöhung des Schrittweitenparameters die Gegenkopplung nicht mit ausreichender Genauigkeit justieren oder den nichtlinearen Bereich der Grenzzyklusschwingung nicht durchfahren. An dieser Stelle ist man auf andere Regelstrategien angewiesen, die jedoch eine genauere Modellierung des Systems voraussetzen.

\section{5 Übertragungsfunktion der Strahl-Kanten-Wechsel- wirkung}

Das durch den adaptiven Algorithmus in den Fixpunkt geregelte System hat die vorgegebene Zielübertragungsfunktion $Z$ erreicht, wenn das Gegenkopplungsfilter adaptiert ist $\left([\Delta C]_{\text {kausal }} \approx\right.$ $0)$. Entspricht die gewählte Zielübertragungsfunktion $Z$ dem Zähler $\left.\left(A S_{1}\right)\right|_{\text {mit Keil }}$ von Gleichung Gl. (5.4), so kompensieren sich gerade die durch die Regelung eingestellte Gegenkopplung und die physikalische Rückkopplung. Die physikalische Rückkopplung $F=\hat{\eta}\left(x_{\text {Regel }}\right) / \hat{\eta}\left(x_{\text {Keil }}\right)$ ist unter der obigen Voraussetzung:

$$
F=\left.A\right|_{\text {mit Keil }} M C .
$$

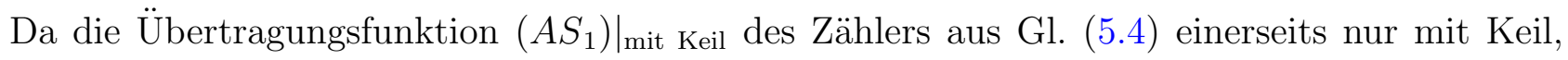
aber ohne dessen Rückwirkung bestimmt werden müsste, kann diese nur im stabilisierten Zustand gemessen werden. Andererseits wird die Dynamik des stabilisierten Zustands durch die Zieldynamik $Z$ festgelegt, die auf der Schätzung von $\left.\left(A S_{1}\right)\right|_{\text {mit Keil }}$ beruht. Damit entzieht sich $\left.\left(A S_{1}\right)\right|_{\text {mit Keil }}$ prinzipiell einer Messung im geschlossenen System mit dieser adaptiven Methode. Daher kann nicht geklärt werden, ob tatsächlich die Gegenkopplung genau der Rückkopplung entspricht, oder ob noch ein Anteil der Gegenkopplung dazu verwendet wird die Abweichung zwischen $Z$ und $A S_{1}$ zu erzeugen. Daher sind die im Folgenden berechneten Übertragungsfunktionen der Strahl-Kanten-Wechselwirkung mit systematischen Fehlern behaftet. Die Messfehler sind dagegen gering, da bei gleichen Strömungsparametern schon kleine Abweichungen der Gegenkopplungen zu weniger Güte bei der Stabilisierung führen und damit ein Gütekriterium zur Verfügung steht.

Die Übertragungsfunktion der Strahl-Kanten-Wechselwirkung $K_{\mathrm{WW}}=\hat{\eta}\left(x_{\mathrm{D}}\right) / \hat{\eta}\left(x_{\text {Keil }}\right)$ von der Streichlinienauslenkung direkt oberhalb des Keils auf die Streichlinienauslenkung direkt unterhalb der Düse ist mit den adaptierten Gegenkopplungsimpulsantworten durch (Gl. (5.14))

$$
K_{\mathrm{WW}}=\hat{\eta}\left(x_{\text {Regel }}\right) /\left.\hat{\eta}\left(x_{\text {Keil }}\right) \cdot A\right|_{\text {mit Keil }} M C=\left.S_{2}{ }^{-1} \cdot A\right|_{\text {mit Keil }} M C
$$


gegeben und mit der Approximation $\left.A\right|_{\text {mit Keil }}=K_{\mathrm{L}} A$ ergibt sich:

$$
K_{\mathrm{WW}}=\left.K_{\mathrm{L}} A\right|_{\text {ohne Keil }} M C S_{2}{ }^{-1} .
$$

In der Berechnung $K_{\mathrm{Ww}}$ wird der Messprozess $M$ durch die Messung von $\left.A\right|_{\text {ohne Keil }}$ (also $\left.\left.A\right|_{\text {ohne Keil }} M\right)$ mitberücksichtig.

Die durch die Stabilisierung gewonnenen Gegenkopplungen werden mit den erreichten Unterdrückungen gewichtet und die Keilübertragungsfunktion $K_{\mathrm{WW}}$ mit der jeweiligen Übertragungsfunktion $S_{2}^{-1}=\hat{\eta}\left(x_{\text {Regel }}\right) / \hat{\eta}\left(x_{\text {Keil }}\right)$ gemäß Gl. (5.16) berechnet. Man erhält die in Abb. 5.18 eingezeichneten Übertragungfunktionen im Vergleich zu $\hat{\eta}\left(x_{\text {Keil }}\right) / \hat{\eta}\left(x_{\mathrm{D}}\right)$ der selbsterregten Moden. Wobei für kleine $L / d$ über viele Messungen gemittelt werden konnte, währenddessen bei $L / d=8$ und der hier betrachteten Reynoldszahl $\operatorname{Re}_{\mathrm{d}}=215$ nur 7 Messungen zur Verfügung standen. Die Gegenkopplungen unterscheiden sich leicht mit der Reynoldszahl, was aber nicht systematisch untersucht wurde.

Der Betrag des Amplitudenverhältnisses zwischen Keil und Düse der natürlichen Moden $\left|\frac{\hat{\eta}\left(x_{\mathrm{D}}\right)}{\hat{\eta}\left(x_{\mathrm{Keil}}\right)}\right|$ nimmt mit wachsendem $L / d$ und damit sinkender $\operatorname{Sr}_{\mathrm{d}}$ zunächst ab (schwarzer Pfeil von (a) nach (b)). Der Sprung von Mode I (a) zu Systemzustand II (b) ist mit einem Sprung zu größeren $\mathrm{Sr}_{\mathrm{d}}$ um den Faktor 2-3 verbunden, mit steigendem $L / d$ verringert sich dann wieder $\operatorname{Sr}_{\mathrm{d}}$ (roter Pfeil), dabei bleibt der Betrag im Wesentlichen konstant.

Zum einen nimmt der Düse-Keil-Abstand zu, so dass das Dipol-Feld $\propto 1 / L^{2}$ von (a) nach (b) um etwa $15 \mathrm{~dB}$ abfällt. Andererseits nimmt die Strahlauslenkung mit größer werdendem Düse-KeilAbstand (siehe Abschnitt 4.5) zu, so dass vermutlich ab einer bestimmten Strahlauslenkung die Strahl-Kanten-Wechselwirkung sättigt und daher noch stärker mit $L / d$ abfällt.

Betrachtet man die Gegenkopplungen, so ist die Abhängigkeit $\propto L^{-2}$ weniger stark ausgeprägt. Für $L / d=8$ wird die Mode II unterdrückt, jedoch ist die Unterdrückung nicht perfekt, wie die der anderen als Keilübertragungsfunktion dargestellten Gegenkopplungen. Die Phase der Keilübertragungsfunktion zeigt zu kleineren Strouhalzahlen einen kleineren Anstieg ähnlich zu $\arg \left(\frac{\hat{\eta}\left(x_{\mathrm{D}}\right)}{\hat{\eta}\left(x_{\text {Keil }}\right)}\right)$. Die Übereinstimmung der Keilübertragungsfunktion mit den Verhältnissen, die man in den natürlichen Moden des Systems vorfindet, zeigt, dass die Zielübertragungsfunktion im Wesentlichen richtig eingestellt wurde.

Als Impulsantwort des Keilfilters $\mathcal{F}^{-1}\left\{K_{\mathrm{Ww}}\right\}$ erwartet man eine kurze um $t=0$ konzentrierte Antwort, da der auftreffende Strahl, so die Vorstellung, ein instantan auf die Düse zurückkoppelndes Druckfeld erzeugt und daher keine größeren Verzögerungen oder Frequenzabhängigkeiten aufweisen sollte. Die durch die Gegenkopplung gemessene Impulsantwort von der Streichlinienauslenkung direkt oberhalb des Keils zu der direkt unterhalb der Düse in Abb. 5.19 ist allerdings zeitlich stark ausgedehnt. Auch die an der Düse stattfindende Umsetzung des Druckfeldes in die Streichlinienauslenkung hat nur eine kleine Verzögerungszeit (Kap. C.2) gegen die zeitiche Ausdehnung der Impulsantwort. Bei der eingestellten Strömungsgeschwindigkeit fängt die Impulsantwort schon bei etwa $-0.5 \mathrm{~s}$ an zu wirken, d.h. die Auslenkung, die vom Aktuator gerade an der Düse erzeugt wird, erzeugt direkt eine Antwort der Strahl-Kanten-Wechselwirkung. 

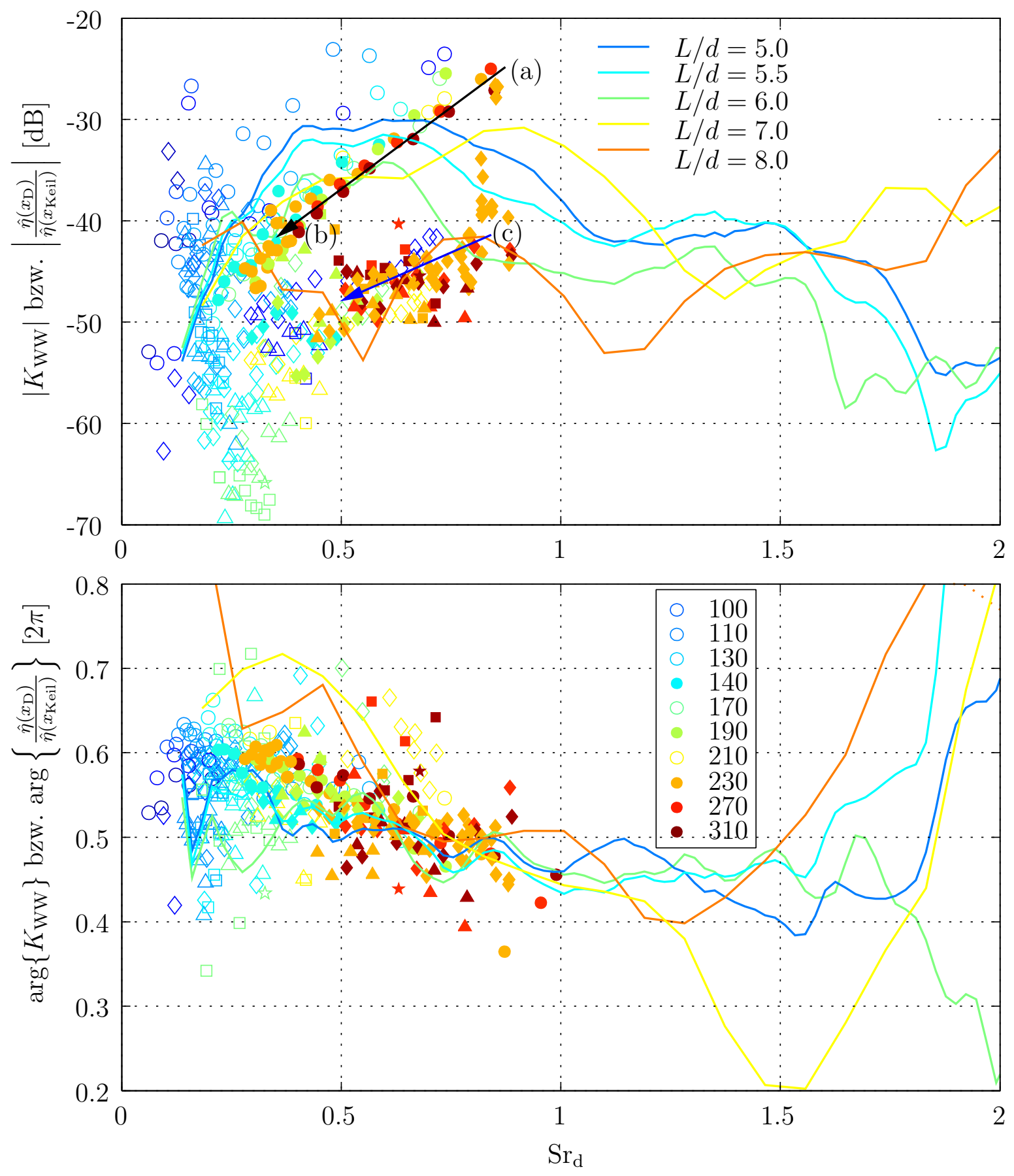

Abb. 5.18: Übertragungsfunktion der Keilrückkopplung durch Messung der Moden und durch Bestimmung aus den Gegenkopplungen verschiedener Messungen. ○ Mode I, $\diamond$ Mode II, $\triangle$ Mode III, $\square$ Mode IV, $\star$ Mode V ; gefüllte Symbole $d=4 \mathrm{~mm}$, sonst $d=2 \mathrm{~mm}$; die Farbe kodiert von blau nach rot die Reynoldszahl. 
Dabei muss allerdings berücksichtigt werden, dass durch den Aktuator die SKWW auch direkt beeinflusst wird, da das Aktuatordruckfeld den Strahl oberhalb des Keils verschiebt und damit abschert. Erstaunlich ist aber die lange Ausdehnung der Impulsantwort für $t>0$ über mehrere Sekunden. Verkürzt man z.B. die Gegenkopplungsimpulsantwort, indem man kürzere Filter benutzt, so ist hier unterhalb einer Impulsantwortlänge von $2 \mathrm{~s}$ keine Stabilisierung möglich. Das unerwartet lange Gedächtnis der Strahl-Kanten-Wechselwirkung kann entweder durch die Regelung selbst entstehen, da das Aktuatordruckfeld die StrahlKanten-Wechselwirkung beeinflussen kann, oder es bildet sich eine Wirbeldynamik auf langen Zeitskalen aus, wie in Zusammen-

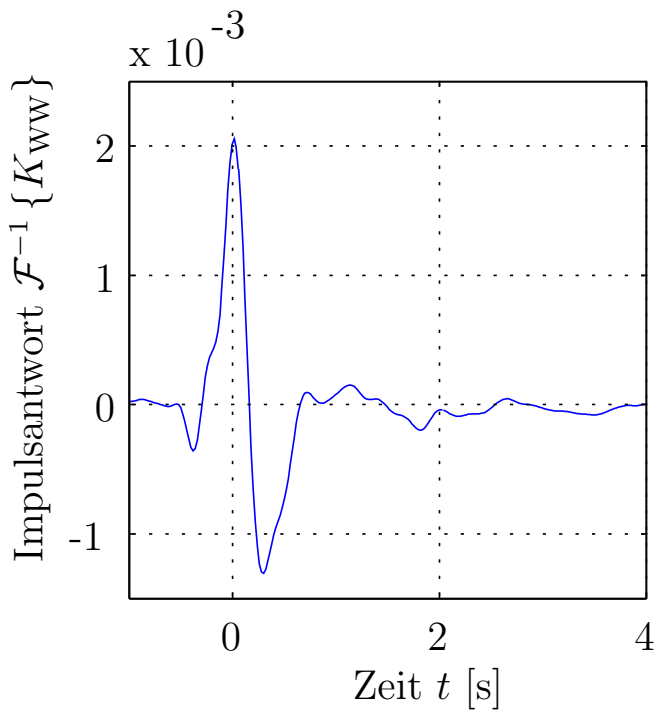

Abb. 5.19: Impulsantwort der Keilübertragungsfunktion $\boldsymbol{K}_{\mathrm{WW}}$ für $L / d=6, U_{\mathrm{D}}=$ $5.36 \mathrm{~cm} / \mathrm{s}, \operatorname{Re}_{\mathrm{d}}=230$ hang mit der Modensynchronisation schon vermutet, so dass die Vorstellung der instantanten Strahl-Kanten-Wechselwirkung falsch ist. 



\section{Kapitel 6}

\section{Zusammenfassung und Ausblick}

Die Dynamik der Strahl-Kanten-Strömung wird detailliert untersucht, insbesondere im Hinblick auf die aktive Strömungsbeeinflussung. Die Instabilität der Strahl-Kanten-Strömung kann mit geeigneten Regelungsmethoden unterdrückt werden. Die Grenze der einzelnen Regelstrategien werden aufgezeigt. Dazu wird ein hochpräzises Mess- und Regelsystem verwendet, das auf der Auswertung der Streichlinienbewegung basiert.

\section{Freistrahlinstabilität}

Mittels der Rayleigh-Gleichung und der WKB-Methode kann das Wechselgeschwindigkeitsfeld der Freistrahlinstabilität in linearer Näherung modelliert werden. Die numerische Streichlinienintegration nähert für kleine Anregungsamplituden die experimentell gemessene gut an. Mit wachsender Anregungsamplitude wird die Anfachung des Geschwindigkeitsfelds und der Streichlinie geringer, und die Phasendrehung pro Laufweg steigt für das Wechselgeschwindigkeitsfeld an, währenddessen die gemessene Streichlinie eine Verringerung der Phasendrehung pro Laufweg zeigt. Dieser Widerspruch kann durch eine Modellierung der Freistrahlinstabilität aufgelöst werden, die die Rückwirkung der Instabilitätswelle auf das sie erzeugende Strahlprofil, insbesondere die daraus resultierende Strahlverbreiterung berücksichtigt.

\section{Modenstruktur und Dynamik der Moden}

Die Moden der unbeeinflussten Strahl-Kanten-Strömung zeigen bei wachsender Reynoldszahl einen Anstieg der mit dem Düse-Keil-Abstand $L$ gebildeten Strouhalzahl. Die Strouhalzahl erreicht erst für $L / d>20$ ( $d$ : Düsenbreite) den theoretisch vorausgesagten $(L / d)^{-1 / 2}$ Abfall. Die Endkorrektur $\epsilon$, die aus einer Schätzung der Phasendrehung der Instabilitätswelle resultiert, ist etwa $-1 / 4$, der Betrag steigt mit wachsendem $L / d$ an.

Die am Keil erreichte Auslenkungsamplitude der Moden steigt im Wesentlichen linear mit $L$ an. Dieses Verhalten kann durch die Sättigung der Freistrahlinstabilität mit wachsender Amplitude plausibel gemacht werden. Die Systemzustände der Strahl-Kanten-Strömung zeigen Hysterese 
und können im Hysteresegebiet durch geeignete Steuersignale umgeschaltet werden. Es werden spontane Übergänge von Systemzuständen beobachtet, deren mittlere Relaxationszeit mehrere 100 Schwingungsperioden beträgt und die nicht auf äußere Einflüsse zurückführbar sind. Die höheren Systemzustände bestehen aus mehreren koexistierenden Moden. Der Anteil der einzelnen Moden an der Gesamtleistung ist etwa gleich, daher definiert die Anzahl der Moden den Systemzustand. Hier werden vor allem die Systemzustände I und II betrachtet. Bei bestimmten Parameterkonfigurationen konkurrieren die Moden, so dass keine der Moden das volle Verstärkungspotenzial des Freistrahls ausnutzen kann. Diese Systemzustände zeichnen sich durch eine kleinere, am Keil erreichte Auslenkungsamplitude aus.

Das Frequenzverhältnis der koexistierenden Moden eines Systemzustandes ist nicht rational und kann bei gleichen Parametern sehr unterschiedlich sein. Die Wechselwirkung zwischen den Moden führt zu einer Synchronisation der Moden innerhalb eines Systemzustandes. Das Einrasten auf einem bestimmten Frequenzverhältnis ist zeitlich nicht konstant, sondern ändert sich auf einer gegen die Synchronisationsperiode langen Zeitskala. Daher besteht das mittlere Frequenzverhältnis aus den entsprechend mit der Zeit des Einrastens gewichteten rationalen Frequenzverhältnissen. Die Synchronisation kann zu rationalen Frequenzverhältnissen führen, die einen großen Zähler und Nenner aufweisen. Das höchste bei der Untersuchung von Systemzustand II gefundene Verhältnis beträgt 47 Schwingungen der höherfrequenten Mode gegen 16 der tieferfrequenten Mode. Diese sehr lange Synchronisationsperiode erfordert einen entsprechend tieffrequenten physikalischen Prozess. Vermutlich ist dieses für die Synchronisation notwendige Gedächtnis durch die Wirbeldynamik gegeben, die durch die vom Keil zerschnittene Strahlwirbeligkeit entsteht. Zusammenfassend kann festgestellt werden, dass die Dynamik der Moden auf drei verschiedenen Zeitskalen stattfindet:

- Frequenz der selbsterregten Schwingung (typischerweise 0.5-4 Hz),

- Frequenz der Synchronisation der Schwingungen (typischerweise 1/3-1/47 der Schwingungsfrequenz),

- Langzeitverhalten der Wirbel in Keilnähe (eine bis mehrere Größenordnungen unter der Synchronisationsfrequenz).

\section{Regelung}

Die Genauigkeit der optischen Streichlinienabtastung wird durch Verringerung des Interpolationsfehler gesteigert, der bei der Positionsbestimmung der Streichlinie zwischen den Bildelementen des CCD-Chips auftritt. Dazu wird das auf dem CCD-Chip entstehende Helligkeitsprofil der Streichlinie durch ein geeignetes Fehlerkriterium geschätzt und parametrisiert. Die Genauigkeit der Aktuation wird durch Invertierung der gemessenen Aktuatorhysterese erhöht.

Mit dem zur Verfügung stehenden Messsystem wird die Strahl-Kanten-Strömung auf die Grenze ihrer Stabilisierbarkeit hin untersucht. Mit steigendem, auf die Düsenbreite normierten Düse- 
Keil-Abstand $L / d$ und wachsender Reynoldszahl $\operatorname{Re}_{\mathrm{d}}$ nimmt die konvektive Verstärkung zu und erfordert eine feinere Justierung des Reglers.

Die Phasenbeziehung zwischen der Streichlinienamplitude an Keil und Düse ist etwa gegenphasig. In einem einfachen Regel-Modell der Strahl-Kanten-Strömung wird dieses Verhältnis genutzt, um die Phase der Gegenkopplung festzulegen. Der Betrag der Gegenkopplung wird durch einen Verstärkungsfaktor eingestellt. Bis zu einem kritischen $L_{\text {krit. }} / d$, oberhalb dessen keine Stabilisierung auf Grund dieses Modells mehr möglich ist, ist ein ganzer Bereich von Verstärkungsfaktoren geeignet, um Strahl-Kanten-Strömung zu stabilisieren. Jedoch verschmälert sich dieser Bereich mit wachsendem $L / d$. Für $L$ in der Nähe von $L_{\text {krit }}$ wechseln sich stabilisierte Systemzustände mit Zuständen ab, in denen das System Schwingungen kleiner Amplitude ausführt. Der Zustandswechsel findet auf einer Zeitskala statt, die lang gegen die natürliche Schwingungsdauer des Systems ist und vermutlich durch die oben erwähnte Wirbeldynamik entsteht.

Die adaptive Regelung basiert auf einer Schätzung der optimal zu erreichenden Dynamik der stabilisierten Strömung. Diese Dynamik wird durch eine Zielübertragungsfunktion $Z$ vom Aktuator zur Messposition charakterisiert, die aus dem System ohne Keil ermittelt wird. Die Gegenkopplung wird sukzessive so bestimmt, dass die Zieldynamik erreicht wird; damit wird die selbsterregte Schwingung unterdrückt. Aus der eingestellten Gegenkopplungsübertragungsfunktion kann die Keilübertragungsfunktion berechnet werden, die das Verhältnis zwischen der Streichlinienamplitude am Keil und an der Düse beschreibt. Ein Vergleich mit dem aus der Modenstruktur gewonnenen Verhältnis zeigt annähernde Übereinstimmung. Damit eine Stabilisierung gelingt muss das Gegenkopplungsfilter eine unerwartet lange Impulsantwort haben.

Als Eingangssignal für die Regelung sind düsennahe Messpositionen besonders geeignet. Die Güte der Stabilisierung kann durch mehrere parallele adaptive Algorithmen von verschiedenen Messpositionen aus bis zur Auflösungsgrenze des Bildverarbeitungssystems gesteigert werden. Die Grenze der Stabilisierbarkeit verschiebt sich dadurch jedoch nicht. Die räumlich hoch korrellierte Streichlinie ist gegenüber anderen den Strömungszustand kennzeichnende Dynamiken, wie beispielsweise großräumige Wirbel, wenig sensitiv.

Die Grenze der Regelbarkeit fällt für die adaptiv eingestellte Gegenkopplung etwa mit dem Übergang von Mode I zu Systemzustands II zusammen. Sie ist sowohl durch eine stark zu $L_{\mathrm{krit}}$ abnehmende Güte der Stabilisierung gekennzeichnet als auch durch eine längere und weniger stabile Adaptionsphase, während das System noch selbsterregt schwingt. Die Grenze liegt für $\operatorname{Re}_{\mathrm{d}} \approx 100$ bei $L_{\text {krit }} / d \approx 10$ und für $\operatorname{Re}_{\mathrm{d}} \approx 200$ bei $L_{\text {krit }} / d \approx 8$. Für die feste Gegenkopplung ist der Bereich um circa 2 Düsenbreiten geringer.

Mit wachsendem $L / d$ und wachsendem $\mathrm{Re}_{\mathrm{d}}$ kann die linearisierte Dynamik des Systems weniger genau bestimmt werden, da die spektrale Leistungsdichte der selbsterregten Schwingungen und ihrer Harmonischen zunimmt. Da die Gegenkopplung von der Schätzung der Zieldynamik abhängt, ist eine genaue Adaption der Gegenkopplung nicht möglich. Selbst wenn sowohl die Systemerkennung als auch die Zielfunktion exakt wären, müsste die adaptive Regelung den 
vorhandenen Schwankungen der Systemdynamik folgen können. Auf welchen Zeitskalen diese Schwankungen stattfinden, ist noch weitgehend ungeklärt. Die vermutete autonome Wirbeldynamik, die durch die selbsterregte Schwingung erzeugt wird, zeigt ihre Auswirkung auch auf die Stabilisierbarkeit des Systems, z. B. wird die Güte der Stabilisierung umso höher, je länger das System schon stabilisiert ist. Daher ist die Annahme einer sehr tieffrequenten Dynamik, die sowohl die Synchronisationszustände als auch die Systemzustände selbst beeinflusst und sich auch negativ auf eine Regelung auswirkt, sehr plausibel.

\section{Ausblick}

Um Aufschlüsse über den Mechanismus der Synchronisation zu erhalten, sollte die Wirbeldynamik in der Nähe des Keils und ihre Auswirkung auf die Strahl-Kanten-Wechselwirkung untersucht werden. Der Einfluss der Wirbeldynamik auf die Streichlinie kann dann abgeschätzt und der Regelung zugänglich gemacht werden.

Im Allgemeinen kann die offene (open-loop) Systemdynamik eines im Grenzzyklus befindlichen rückgekoppelten Systems nicht geschätzt werden. Daher ist die hier erforderliche optimale Wahl der Zieldynamik ein Hindernis bei der Verallgemeinerung dieses Algorithmus. Zur Schätzung der Zieldynamik während der Messung ist es möglich, wie in Gl. (5.11) zunächst eine Gegenkopplung ohne Zielfunktion zu berechnen. Aus der Änderung der Systemdynamik durch die Gegenkopplung könnte mit Hilfe eines Gütekriterium für die Zieldynamik, wie z. B. minimale Laufzeit und möglichst flaches Spektrum, die Zielfunktion $Z$ adaptiv berechnet werden. Weiterführende Untersuchungen sollten besonderes Augenmerk auf die akausalen Anteile der inversen Übertragungsfunktion des geschlossenen Systems $\left(H^{(g)}(C)^{-1}\right)$ legen, da beobachtet werden konnte, dass sich diese in der Nähe der optimalen Zielfunktion stark ändern. Das Gütekriterium muss so in den Algorithmus integriert werden, dass die Stabilität während der Adaption gewährleistet bleibt.

Eine der ungelösten Fragen ist, welche Information notwendig ist, um das System bezüglich der Regelung zu charakterisieren. Der Verdacht liegt nahe, dass die Streichlinienauslenkung sich hier weniger eignet, da sie die physikalisch wichtigen Wechselgeschwindigkeitsfelder nichtlinear abbildet. Daher ist es sinnvoll, mit PIV oder LDA ein System aufzubauen, dass direkten Einblick in das Strömungsfeld erlaubt.

Wenn man sich der Frage der prinzipiellen Grenze der Stabilisierbarkeit weiter nähern will, muss eine genauere Modellierung des Systems mit allen Nichtlinearitäten erfolgen. Daraus könnte sich eine erfolgreiche, nichtlineare Regelstrategie ergeben. 


\section{Anhang A}

\section{A.1 Stabilität der Lösung der Rayleighgleichung ge- genüber kleinen Profilstörungen}

Im Folgenden soll die Stabilität der Wellenzahl $k$ bezüglich leicht gestörter Gleichströmungsprofile $\bar{U}(y)$ untersucht werden. Zu dem glatten Gleichströmungsprofil $\bar{U}(y)$ wird eine Störung

$$
\bar{U}_{\mathrm{r}}(y)=\bar{U}(y)(1+A \cdot r(y)),
$$

addiert. Dabei ist $r(y)$ ein in der Raumfrequenz bandbegrenztes Rauschen mit der mittleren räumlichen Variationswellenzahl $k_{\mathrm{y}}^{\mathrm{r}}$. Die durch $r(y)$ hervorgerufene Änderung von $k$ hängt sehr stark von $k_{\mathrm{y}}^{\mathrm{r}}$ ab. Ist $k_{\mathrm{y}}^{\mathrm{r}}$ wesentlich größer als die räumliche Wellenzahl der resultierenden Wechselgeschwindigkeit $\tilde{v}(y)$, so wird $k$ sehr wenig durch $r(y)$ beeinflusst. Bei kleineren $k_{\mathrm{y}}^{\mathrm{r}}$ wird die Streuung von $k$ größer. Die Rayleighgleichung (Gl. (3.3)) hat also eine Art „Tiefpasscharakteristik" bezüglich der Übertragung der gestörten Strömungsprofile nach Variationswellenzahlen (Urbildmenge) zerlegt auf die resultierenden Wellenzahlen $k$ (Bildmenge). Die Lösung $\tilde{v}(y)$ selbst weist eine größere standardisierte Streuung auf als $k$.

In Abb. A.1 ist die relative Standardabweichung (RSD) der Wellenzahl $\sigma\{k\} /\langle k\rangle$ bezüglich der relativen Standardabweichung der Störung $r(y)$ des Gleichströmungsprofils für verschiedene Variationswellenzahlen $k_{\mathrm{y}}^{\mathrm{r}}$ bezogen auf die Düsenbreite aufgetragen. Dazu werden in Abb. A.1 in jedem räumlichen Wellenzahlband mit der Mittenwellenzahl $k_{\mathrm{y}}^{\mathrm{r}}$ jeweils 100 verschiedene $r(y)$ mit derselben Bandpasscharakteristik erzeugt, dann die Rayleigh-Gleichung für $\operatorname{Sr}_{d}=0.75$ gelöst und über die Ensemble der dazu gefundenen Lösungen $k(r)$ die Standardabweichungen für die verschiedenen Wellenzahlbänder $k_{\mathrm{y}}^{\mathrm{r}}$ berechnet. Für kleine Störamplituden stellt sich ein linearer Zusammenhang der Standardabweichung von der Störamplitude ein, wie in Abb. A.1 gezeigt. Die typische räumliche Wellenlänge der zu den Gleichströmungsprofilen gehörenden Lösung $\tilde{v}(y)$ entspricht etwa 0.8 Düsenbreiten $d$, was in Abb. A.2 gezeigt ist. Die Variationswellenzahlen sind auf die Düsenbreite bezogen. Das Maximum der Störanfälligkeit wird erreicht, wenn $k_{\mathrm{y}}^{\mathrm{r}}$ etwa doppelt so groß ist, wie die charakteristische Wellenzahl der Lösung $\tilde{v}(y)$. 
120Anhang A. Anhang A: Stabilität der Lösung der Rayleighgleichung gegenüber kleinen Profilstörungen

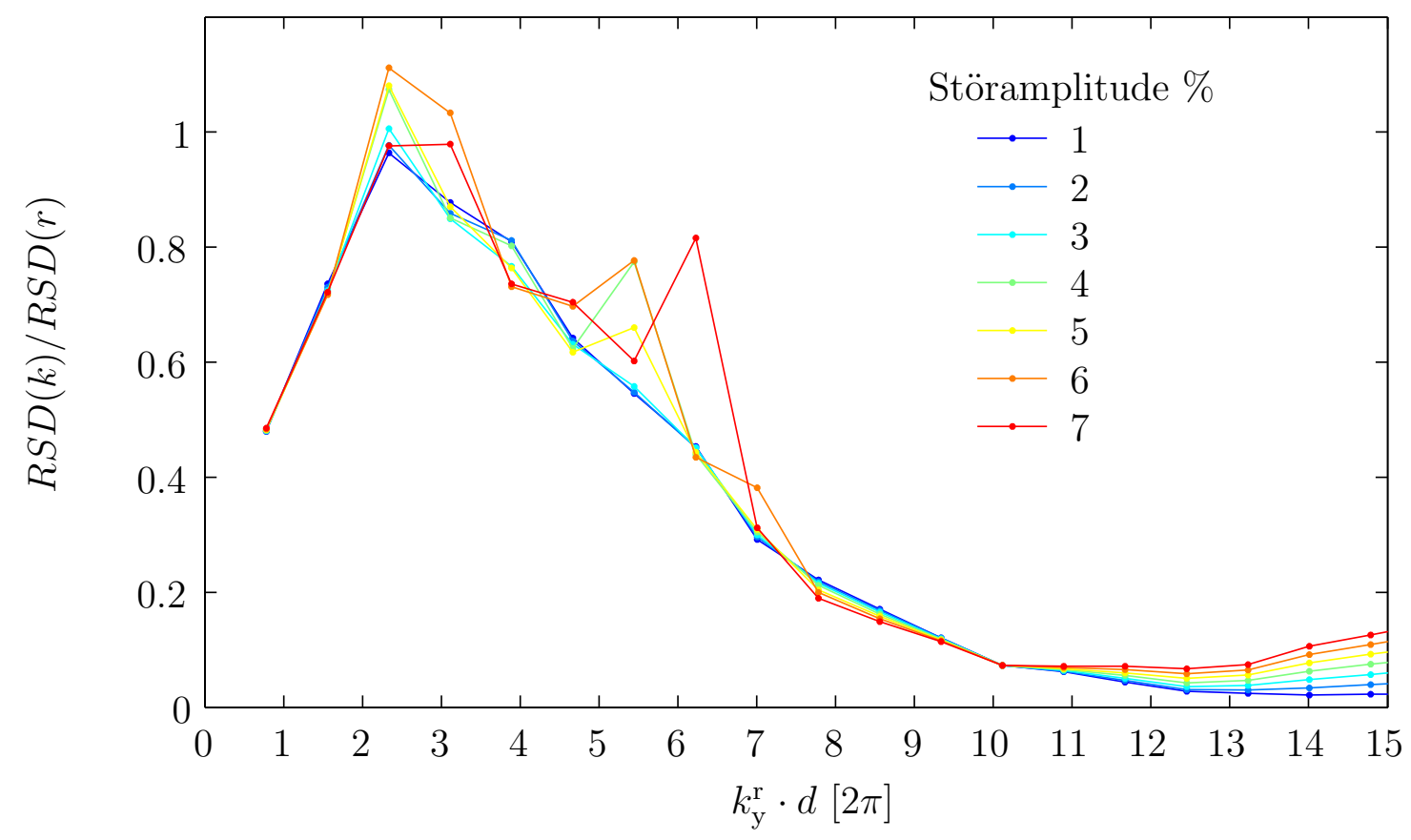

Abb. A.1: Tiefpassverhalten der Rayleighgleichung gegenüber statistischen Fluktuationen des Gleichströmungsprofils. Als charakeristische Ausdehnung $d$ (in $y$-Richtung) wurde die Düsenbreite gewählt.

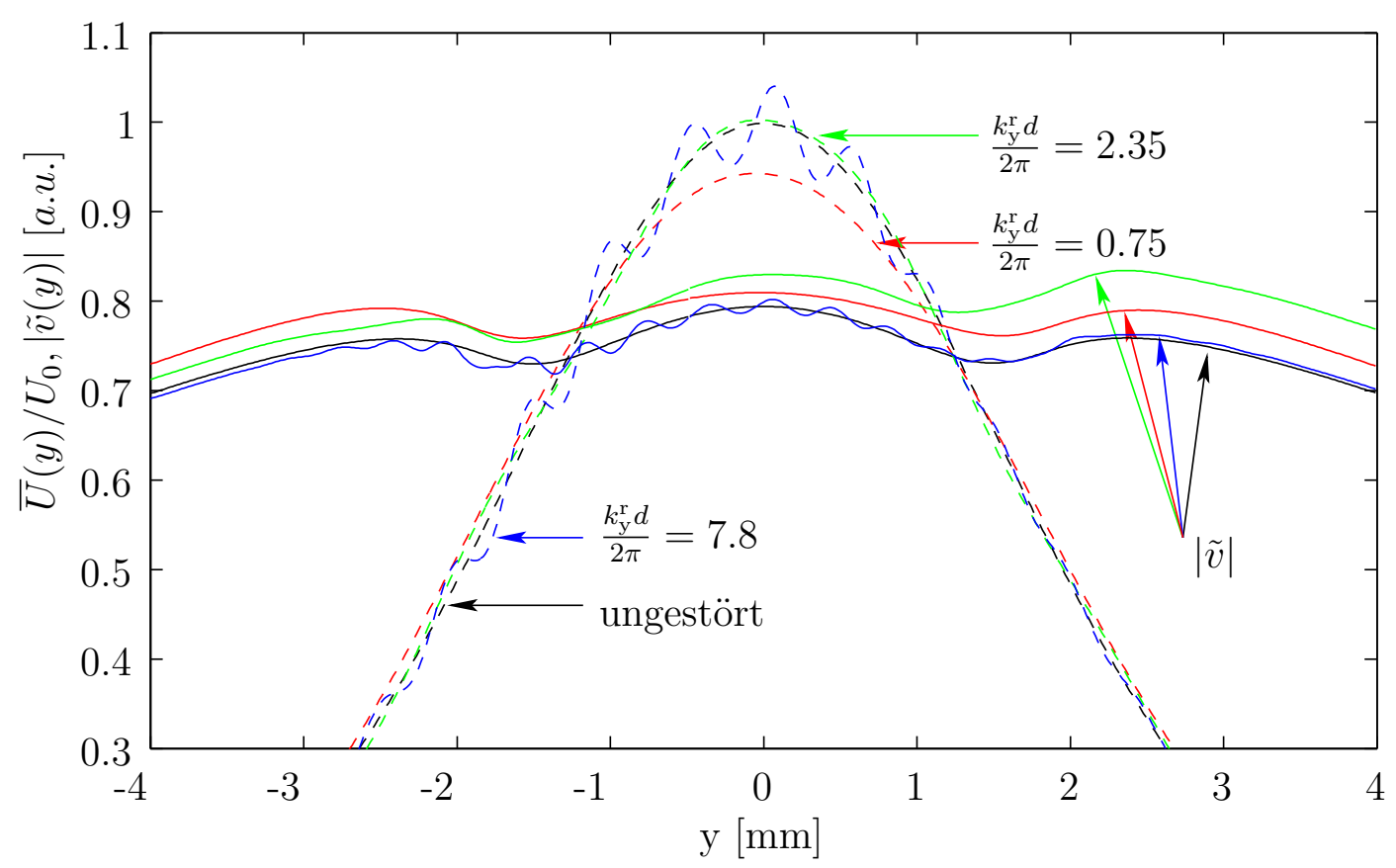

Abb. A.2: Gestörte Gleichströmungsprofile und zugehörige Wechselgeschwindigkeit für verschiedene Variationswellenzahlen. Störamplitude $A=6 \%$, Düsenbreite $d=4 \mathrm{~mm}$. 


\section{Anhang B}

\section{B.1 Streichlinien und Sättigungsverhalten der Strahl- instabilität}

In den ersten beiden Abschnitten wird jeweils ein Ansatz zur Berechnung der Profilverbreiterung vorgestellt, mit der eine Sättigung der Anfachung modelliert werden soll. Diese Art der Nichtlinearität führt unter anderem zu dem räumlichen Anwachsverhalten der Moden der globalen Instabilität (Kap. 4.9).

In den darauf folgenden Abschnitten wird die Übertragung der Wechselfeldamplitude auf die Streichlinienamplitude auf ihren Linearitätsbereich hin untersucht. Dabei wird auch abgeschätzt, welchen Einfluss eine laterale Versetzung der Streichlinie auf die Übertragungsfunktion zwischen zwei Orten auf dem Strahl hat.

\section{B.1.1 Verbreiterung durch die Rückwirkung der Wechselschub- spannungen auf das Gleichströmungsprofil}

Das mittlere Strömungsfeld $\bar{U}(x, y)$ wird durch die Reynoldssche Schubspanungen

$$
\bar{\tau}_{\text {Inst. }}\left(x, y, \omega_{\mathrm{s}}\right)=-\frac{1}{2} \mathfrak{R e}\left(\hat{u}\left(x, y, \omega_{\mathrm{s}}\right) \hat{v}\left(x, y, \omega_{\mathrm{s}}\right)^{*}\right)
$$

aufgrund der Anwesenheit der Instabilitätswelle verbreitert. Da ständig Energie von der Gleichströmung in die Wechselströmung fließt, muss $\left\langle\frac{\partial \bar{U}}{\partial y} \bar{\tau}_{\text {Inst. }}\right\rangle_{y}$ positiv sein, und durch partielle Integration folgt daraus $\left\langle\bar{U} \frac{\partial \bar{\tau}_{\text {Inst. }}}{\partial y}\right\rangle_{y}<0$. Da fast überall $\bar{U}>0$ ist, wird die Strömung im Mittel abgebremst, d.h. $\frac{\partial \bar{\tau}_{\text {Inst. }}}{\partial y}<0$ für $\bar{U}>0$. Betrachtet man die Strömung im stationären Zustand und vernachlässigt mögliche Druckgradienten gegen den Einfluss der Schubspannung, so kann man mit Hilfe der folgenden iterativ angewendeten Impulsgleichung

$$
\begin{aligned}
\bar{U}^{(\mathrm{n}+1)}(x, y) \frac{\partial \bar{U}^{(\mathrm{n}+1)}(x, y)}{\partial x} & =\frac{\partial \bar{\tau}_{\text {Inst. }}^{(\mathrm{n})}(x, y)+\tau_{\nu}(x, y)}{\partial y} \\
\text { mit Anfangsbedingung } \bar{U}^{(\mathrm{n})}(x=0, y) & =\bar{U}(x=0, y)
\end{aligned}
$$




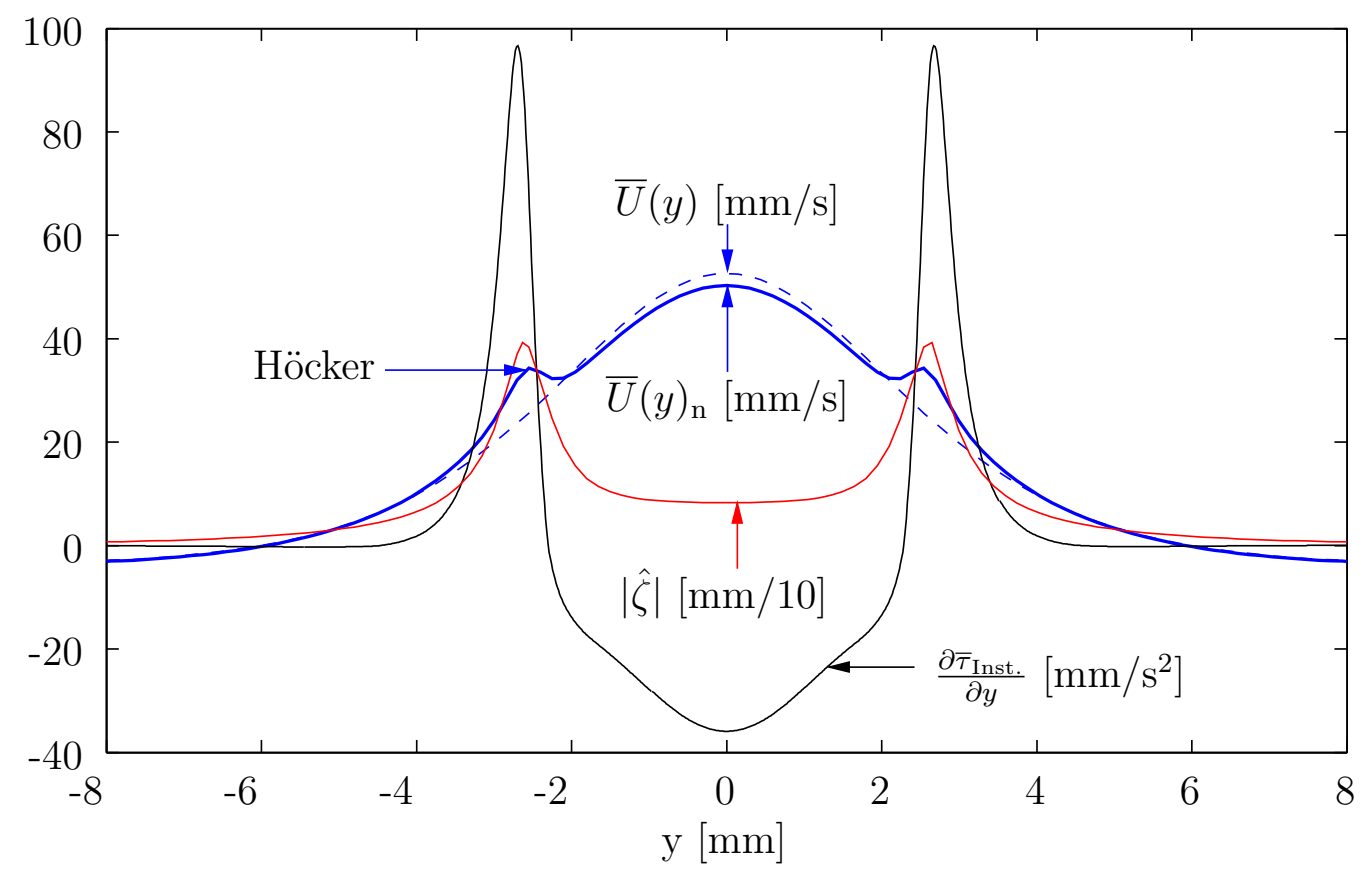

Abb. B.1: Durch Schubspannungen verformtes Gleichströmungsprofil.

das sich einstellende Gleichströmungsfeld berechnen. Der obere Suffix bezeichnet den Iterationsschritt, $\bar{\tau}_{\text {Inst. }}$ ist die mit der jeweiligen Rayleigh-Gleichung berechnete Schubspannungsverteilung und $\tau_{\nu}$ die viskose Schubspannung. Die viskose Schubspannung $\tau_{\nu}$ wird vorher aus dem Profil $\bar{U}$ mittels der Gl. (B.2) ohne die Wechselschubspannungen berechnet. Zu jedem $\bar{U}^{(\mathrm{n})}$ gehören neue Eigenfunktionen $\tilde{u}, \tilde{v}$, die durch die WKB-Methode bis auf einen Faktor bestimmt sind. Dieser Amplitudenfaktor $A_{0}$ (siehe Gl. (3.4)) muss der Aktuatoramplitude durch Vergleich mit dem Experiment zugeordnet werden und bleibt während der Iteration konstant. Zunächst wählt man $A_{0}$ so, dass die experimentelle und die numerisch integrierte Streichlinie bei kleinen Aktuatoramplituden etwa übereinstimmen. Daraus lässt sich die korrigierte Wechselschubspannung

$$
\bar{\tau}_{\text {Inst. }}^{(\mathrm{n})}=-\frac{1}{2} A_{0}^{2} \mathfrak{R e}\left(\hat{u}\left(x, y, \omega_{\mathrm{s}}\right) \hat{v}\left(x, y, \omega_{\mathrm{s}}\right)^{*}\right)
$$

berechnen, mit der man wiederum ein neues Gleichströmungsprofil $\bar{U}^{(\mathrm{n}+1)}$ ausrechnen kann und so fort, wie hier dargestellt:

\begin{tabular}{|c|c|c|c|c|}
\hline \multicolumn{3}{|c|}{$A_{0}$ wird festgelegt, $\tau_{\nu}$ aus $\bar{U}^{(0)}$ bestimmt } & \multirow[b]{2}{*}{ Gl. (B.4) } & \multirow[b]{2}{*}{$\bar{\tau}_{\text {Inst. }}^{\text {(n) }}$} \\
\hline $\bar{U}^{(\mathrm{n})}$ & WKBJ Gl. (3.6) & $\tilde{u}^{(\mathrm{n})}, \tilde{v}^{(\mathrm{n})}$ & & \\
\hline A & Gl. ( & $n \rightarrow n+1$ & & \\
\hline
\end{tabular}

Hat man am Ende der Iteration ein auskonvergiertes Gleichströmungsprofil für den Amplitudenfaktor $A_{0}$ gefunden, so lässt sich die gewonnene Wechselströmung und damit auch die daraus 
errechnete Streichlinie mit den experimentellen Streichlinien vergleichen.

Insgesamt erhält man aus diesem Prozess eine Verbreiterung des mittleren Strömungsprofils. $\mathrm{Da}\left\langle\frac{\partial \bar{\tau}_{\text {Inst. }}}{\partial y}\right\rangle_{y}$ negativ ist bei überwiegend positivem $\bar{U}$, führt dies zu einem negativen $\frac{\partial \bar{U}}{\partial x}$. Die Strömung wird somit abgebremst, und es zeigt sich, dass an Orten, für die $\bar{U}$ negativ ist, $\bar{U}$ stromab beschleunigt wird.

Ein erstes Problem besteht darin, dass die Schubspannung $\bar{\tau}_{\text {Inst. }}(x, y)$ an den Stellen, an denen $\bar{U}(x, y)$ null ist, nicht exakt verschwinden. Dies führt bei der Integration zu Singularitäten, da an den Nullstellen des Strömungsprofils eine unendlich kleine Kraftdichte die Strömung beschleunigen kann. Daher wird $\bar{\tau}_{\text {Inst. }}$ einfach an den Nullstellen des Strömungsprofils auf null gesetzt.

Ein schwierigeres Problem bereitet die kritische Schicht. In einer linearisierten Störungsrechnung reduziert sich der Term $\frac{\partial U}{\partial t}+U \frac{\partial U}{\partial x} \mathrm{zu} i k(\bar{U}-c) \tilde{u}$ (mit $c=\omega / k$ der Phasengeschwindigkeit der Instabilitätswelle). Wenn $\bar{U}=c$ bei $y=y_{\mathrm{c}}$ ist, so laufen in dieser kritischen Schicht $y_{\mathrm{c}}$ die Wellen mit genau derselben Geschwindigkeit wie die Gleichströmung. Somit ist die Wechselwirkung zwischen Welle und Gleichströmung dort besonders stark. Die Schubspannungen können zu einer lokal starken Anhebung der Strömungsgeschwindigkeit führen. Man erhält typischerweise an diesen Stellen Höcker auf dem $\bar{U}$-Profil (siehe Abb. B.1), die darauf zurückzuführen sind, dass hier die Viskosität [22] und die Nichtlinearität [28] vernachlässigt wurden und somit $\tilde{u}$ unstetig ist. Im Abbildung B.1 ist die Auslenkung $\hat{\zeta}$ in der kritischen Schicht $4 \mathrm{~mm}$, im Experiment würde man die in der Mitte berechneten $0.8 \mathrm{~mm}$ messen. Durch die Linearisierung der Eulergleichung wird ein Fluidteilchen nicht aus der kritischen Schicht fortbewegt, so dass dort große Auslenkungen $\hat{\zeta}$ entstehen. Die nichtlinearen Glieder sorgen für eine Durchmischung, so dass ein Teilchen nur kurze Zeit in der kritischen Schicht verweilt und so der Einfluss der kritischen Schicht kleiner wird. Ohne Berücksichtigung der Nichtlinearität führt dieser Ansatz also zu einer falschen Verbreiterung des Profils. Daher wurde der Versuch, die nichtlineare Profilentwicklung direkt aus den Reynoldsschubspannungen zu berechnen, verworfen. 


\section{B.1.2 Verbreiterung des Gleichströmungsprofils durch die Mitte- lung des instationär verschobenen Gleichströmungsprofils}

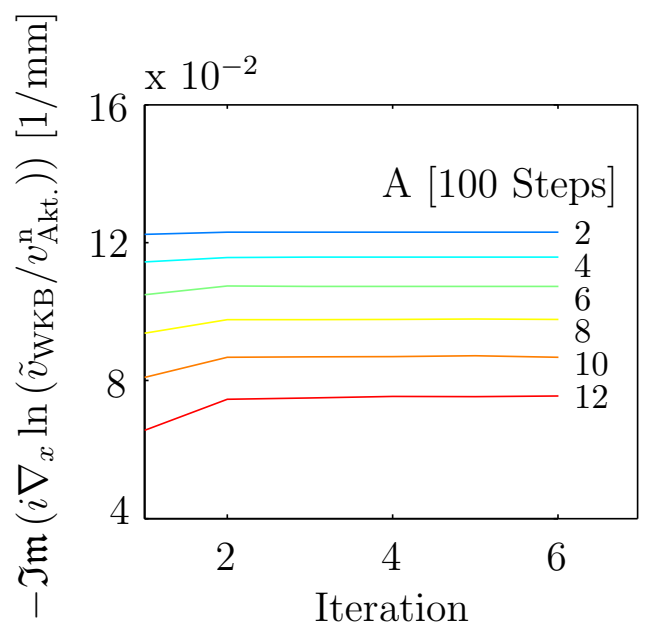

Abbildung B.2: Konvergenz der Anfachung für verschiedene Aktuatoramplituden $\left(f_{\mathrm{s}}=2 \mathrm{~Hz}, x\right.$-Position $\left.32 \mathrm{~mm}\right)$.

Stellt man sich vor, dass das Gleichströmungsfeld durch die Instabilitätswelle periodisch sinusförmig lateral versetzt wird, so kann man die Profilverbreiterung durch Mittelung über die verschobenen Gleichströmungsprofile abschätzen. Die seitliche Auslenkung der Fluidelemente auf der Mittellinie des Strahls $(y=0)$ kann als Näherung für die Auslenkung des gesamten Strahls betrachtet werden. Damit umgeht man das Problem der großen Auslenkungen in der kritischen Schicht. Dieses sehr einfache Modell der instationären Strömung führt zu brauchbaren qualitativen Ergebnissen (siehe Kap. 3.6). Die Auslenkung kann durch

$$
\begin{aligned}
\tilde{\zeta}(x) & =e^{-i \omega_{\mathrm{s}} \int_{x_{\mathrm{D}}}^{x} \bar{U}(x, 0)^{-1} d x^{\prime}} \\
& \cdot \int_{x_{\mathrm{D}}}^{x} \frac{e^{i \omega_{\mathrm{s}} \int_{x_{\mathrm{D}}}^{x^{\prime}} \bar{U}(x, 0)^{-1} d x}}{\bar{U}\left(x^{\prime}, 0\right)} \tilde{v}\left(x^{\prime}, y \equiv 0\right) d x^{\prime}
\end{aligned}
$$

bestimmt werden. Das verbreiterte Profil wird nun durch Faltung mit der Amplitudenverteilung der Sinusfunktion, also durch:

$$
\bar{U}_{\text {Inst. }}\left(x, y,\left|\hat{a}_{\mathrm{Akt} .}\right|\right)=\frac{1}{\pi} \int_{-\infty}^{\infty} \bar{U}\left(x, y-y^{\prime}\right) \frac{1}{\sqrt{\left.\left|\hat{\zeta}\left(x,\left|\hat{a}_{\mathrm{Akt.}}\right|\right)\right|^{2}-y^{\prime 2}\right)}} d y^{\prime}
$$

berechnet werden. Man erhält so für jede Anregungsamplitude ein anderes Gleichströmungsfeld. Dieses Verfahren kann, genauso wie oben beschrieben, iterativ angewendet werden, d.h. es kann immer wieder eine korrigierte Auslenkungsamplitude $\hat{\zeta}$ in Abhängigkeit der Aktuatoramplitude $\left|\hat{a}_{\text {Akt. }}\right|$ gewonnen werden. Da die Auslenkungsamplitude im ersten Schritt überschätzt wird, kann ein schnellere Konvergenz durch eine kleinere Wahl der Auslenkungsamplitude erreicht werden (Abb. B.2). Durch die Verbreiterung des Profils erhöht man die lokale Strouhalzahl. Damit wird die Anfachung für hohe Frequenzen mit steigender Amplitude kleiner; für tiefe Frequenzen wächst die Anfachung erst bis zu einer Strouhalzahl der maximalen Anfachung an. Daher werden die tiefen Frequenzen bei größerer Amplitude bis zu einer gewissen Stromabposition besser übertragen. 


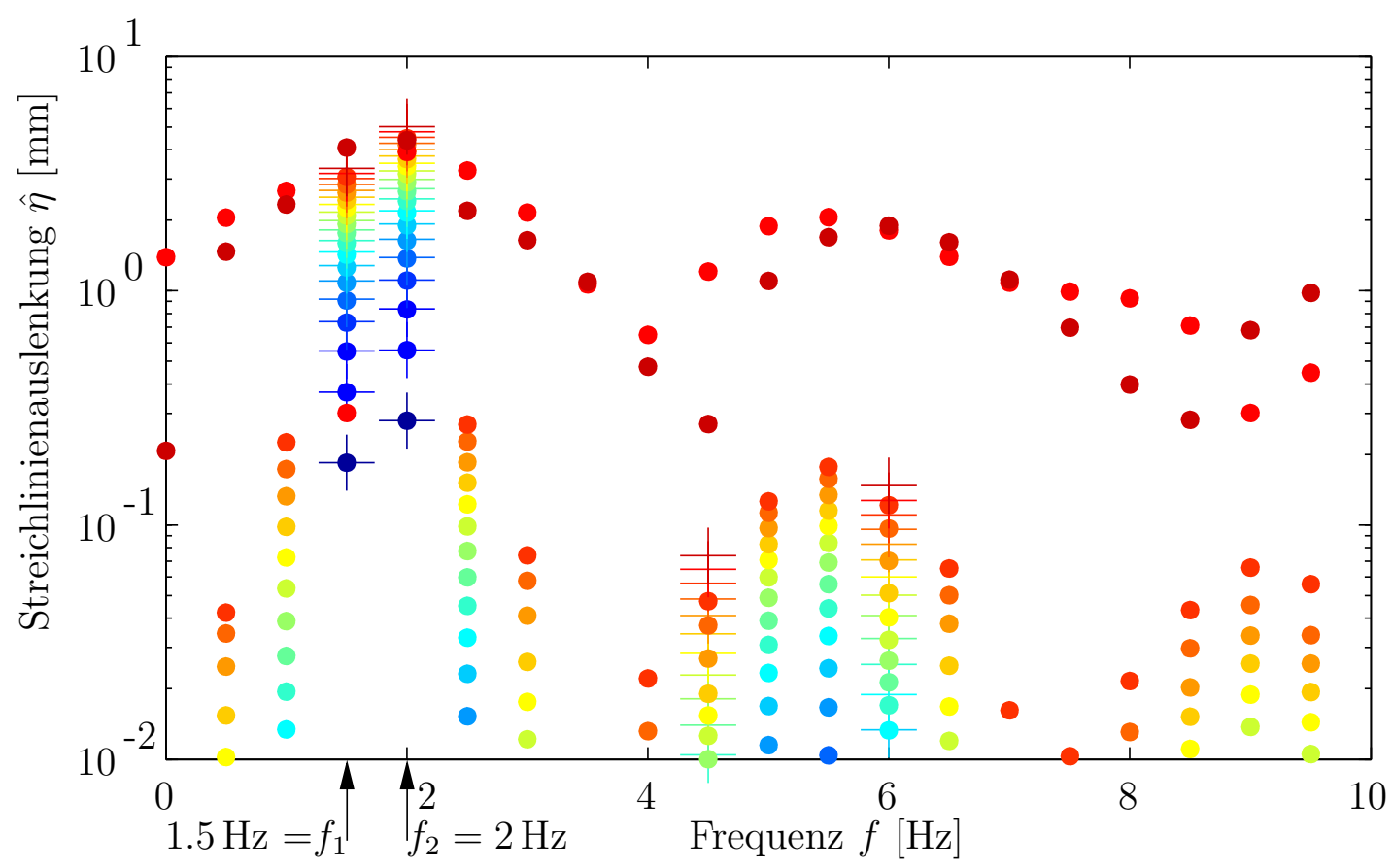

Abb. B.3: Mischung zweier Frequenzen durch Streichlinienintegration. Die Punkte kennzeichnen die Streichlinie im überlagerten Feld. Die Aktuatoramplitude steigt äquidistant von blau nach rot von 50 Steps zu 850 Steps. Die Streichlinienauslenkung wird am Ort $x=20 \mathrm{~mm}$ betrachtet. Die Kreuze sind die Spektralkomponenten, die bei der Integration der einzelnen Frequenzen entstehen.

\section{B.1.3 Streichlinienintegration bei Überlagerung zweier verschieden frequenter Geschwindigkeitsfelder}

Die Überlagerung zweier Wechselfelder mit unterschiedlichen Anregungsfrequenzen und der daraus integrierten Streichlinie unterscheidet sich für größere Amplituden erheblich von der Überlagerung der zu den einzelnen Wechselfeldern gehörenden Streichlinien.

Das zu einem periodischen Zeitsignal $s(t)=\sum \hat{S}\left(f_{\mathrm{k}}\right) \exp \left(i 2 \pi f_{\mathrm{k}}\right)$ gehörende (vektorielle) Strömungsfeld $\underline{U}(x, y, t)$ setzt sich im linearen Fall additiv aus den Wellenlösungen $\underline{\tilde{u}}\left(x, y, f_{\mathrm{k}}\right)$ für die jeweilige Frequenz zusammen:

$$
\underline{U}(x, y, t)=\underline{\bar{U}}+\sum_{k=1}^{n} \hat{S}\left(f_{\mathrm{k}}\right) \underline{\hat{u}}\left(x, y, f_{\mathrm{k}}\right) e^{i 2 \pi f_{\mathrm{k}} t} \operatorname{mit} \underline{\bar{U}}=(\bar{U}, \bar{V})^{t} .
$$

Aufgrund der hier diskutierten Nichtlinearität sind dann in diesem Streichliniensignal $\eta(x, t)$ 
alle Mischfrequenzen

$$
\sum_{k=1}^{n} l_{\mathrm{k}} f_{\mathrm{k}} \quad l_{\mathrm{k}} \in \mathcal{Z}
$$

enthalten. Für den Fall $n=2, f_{1}=1.5 \mathrm{~Hz}, f_{2}=2 \mathrm{~Hz}$ sind in Abb. B.3 für verschiedene Aktuatoramplituden, $\left|\hat{a}_{\text {Akt. }}\left(f_{1}\right)\right|=\left|\hat{a}_{\text {Akt. }}\left(f_{2}\right)\right|=50,100, \ldots, 850$ Steps, die spektralen Amplituden bei den verschiedenen Mischfrequenzen dargestellt und mit den Spektren verglichen, die sich aus der linearen Übertragung $\eta\left(x, t, f_{1}\right)+\eta\left(x, t, f_{2}\right)$ ergeben. Für Amplituden bis circa 300 Steps Aktuatorauslenkung ist die Linearität etwa gegeben, d.h. die entstehenden Mischfrequenzen spielen noch keine wesentliche Rolle. Da hier ein komensurables Frequenzverhältnis $(1.5 \mathrm{~Hz}$ und $2 \mathrm{~Hz}$ ) gewählt wurde, sieht man nicht genau welche Frequenzkomponenten von welcher Anregungsfrequenz herrühren. Die spektralen Amplituden bei den beiden Anregungsfrequenzen entsprechen sehr genau den Amplituden von $\eta\left(x, t, f_{1}\right)+\eta\left(x, t, f_{2}\right)$. Die Amplitude der 1. Harmonischen und der 3. Harmonischen sind in der Mischung etwas abgesenkt, dafür entstehen Spektralkomponenten bei allen Vielfachen der Differenzfrequenz. Bei besonders großen Amplituden $(>800$ Steps) rollt sich die Streichlinie auf, und es entsteht eine sehr starke nichtlineare Verzerrung (siehe sprunghaften Anstieg der Mischfrequenz-Amplituden in Abb. B.3).

\section{B.1.4 Höher-harmonische Spektralkomponenten als Maß für die Nichtlinearität}

Die nichtlineare Reaktion der experimentell gemessenen Streichlinienauslenkung auf das Aktuatorsignal spiegelt sich in den höher-harmonischen Spektralkomponenten wider. Diese kann man mit den Harmonischen vergleichen, die numerisch durch die Integration der Streichlinie auf Basis des verbreiterten Hauptströmungsprofils und einem linear berechneten Wechselprofils erzeugt werden. In Abb. B.4 sind die Harmonischen des Spektrums relativ zur ersten Harmonischen als Funktion der Aktuatoramplitude aufgetragen. Die Nichtlinearität der gemessenen Streichlinie ist deutlich größer als die der berechneten.

Im Gegensatz dazu rollt sich die gemessene Streichlinie bei $2 \mathrm{~Hz}$ und $x<30 \mathrm{~mm}$ erst für $\left|\hat{a}_{\text {Akt. }}\right|>1500$ Steps auf, während die numerisch bestimmte Streichlinie sich schon bei $\left|\hat{a}_{\text {Akt. }}\right|>450$ Steps rückwärts neigt. Zum einen wächst die numerisch bestimmte Streichlinienauslenkung bei größeren Amplituden im Vergleich zum Experiment zu stark an. Die im Abschnitt B.1.2 abgeschätzte korrigierte Anfachung der Instabilitätswelle müsste demnach noch stärker mit wachsender Auslenkungsamplitude abfallen, als dies in Abb. B.2 gezeigt ist. Zum anderen ist die kritische Schicht aufgrund der in Abschnitt B.1.1 beschriebenen Vernachlässigungen unzulänglich behandelt. Durch das dortige Verweilen der Fluidelemente ändert die Streichlinie ihre Richtung (Abb. 3.8). 


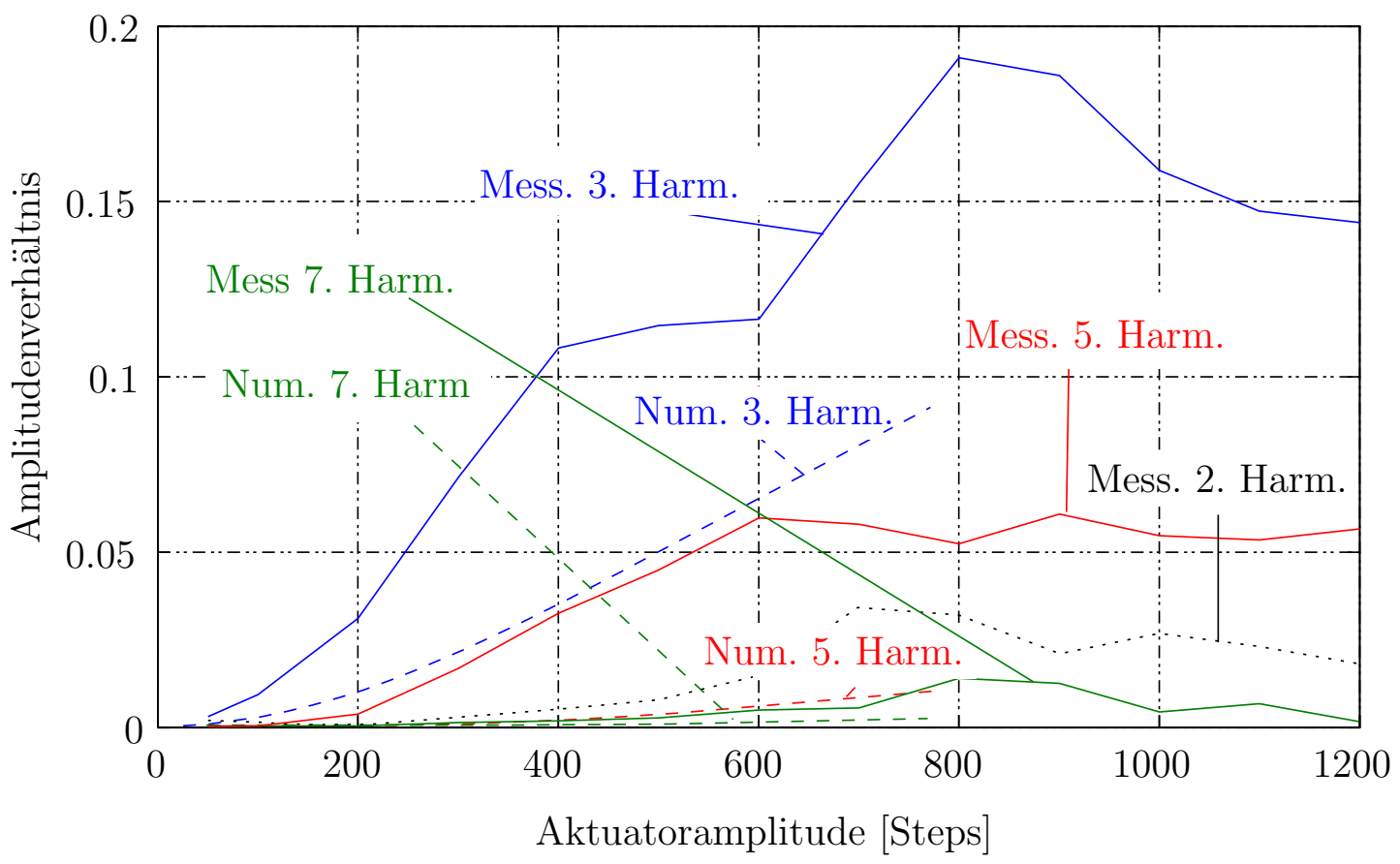

Abb. B.4: Amplitudenverhältnis der n-ten Harmonischen zur 1. Harmonischen der Streichlinienauslenkung am Ort $x=30 \mathrm{~mm}$.

\section{B.1.5 Streichlinienauslenkung in Abhängigkeit von der mittleren Position der Streichlinie}

Im Experiment liegt die eingefärbte Streichlinie nicht immer in der Düsenmitte, da langsame Fluktuationen im Einlaufbereich des Düsentrichters die Lamelle mit Frequenzen unter $0.3 \mathrm{~Hz}$ auslenken. Damit erhält man eine von der $y$-Position abhängige Übertragungsfunktion $\hat{\eta} / \hat{a}_{\text {Akt. }}$, die wegen der dann bestehenden Asymmetrie so verzerrt wird, dass auch die 2. Harmonische im Spektrum auftaucht.

Bei der adaptiven Regelung der Strahl-Kanten-Strömung wird meistens ein Sweep-Signal (Schröder-Sweep) benutzt, um die Systemparameter zu ermitteln. Dazu werden hier insbesondere die spektralen Amplitudenverhältnisse $\hat{\eta}\left(x_{2}, f\right) / \hat{\eta}\left(x_{1}, f\right)$ der Streichlinienauslenkung zwischen verschiedenen $x$-Positionen $x_{2}>x_{1}$ bei kleinen Anregungsamplituden gemessen, die bei Sweep-Anregung etwas kleiner ausfallen als bei sinusförmiger Anregung. Diese Amplitudenverhältnisse werden hier als Übertragungsfunktion bezeichnet, obwohl sie nicht notwendigerweise eine Ursache-Wirkung-Beziehung beschreiben, d.h. man kann nicht erwarten, dass die Fourierrücktransformation für negative Zeiten verschwindet (Abschnitt 3.7).

In Abb. B.5 ist die Übertragungsfunktion $\hat{\eta}\left(x_{2}, f\right) / \hat{\eta}\left(x_{1}, f\right)$ in Abhängigkeit von der lateralen $y$-Startposition der Streichlinienintegration im Vergleich zu einer Sweep-Messung in der Strahl- 


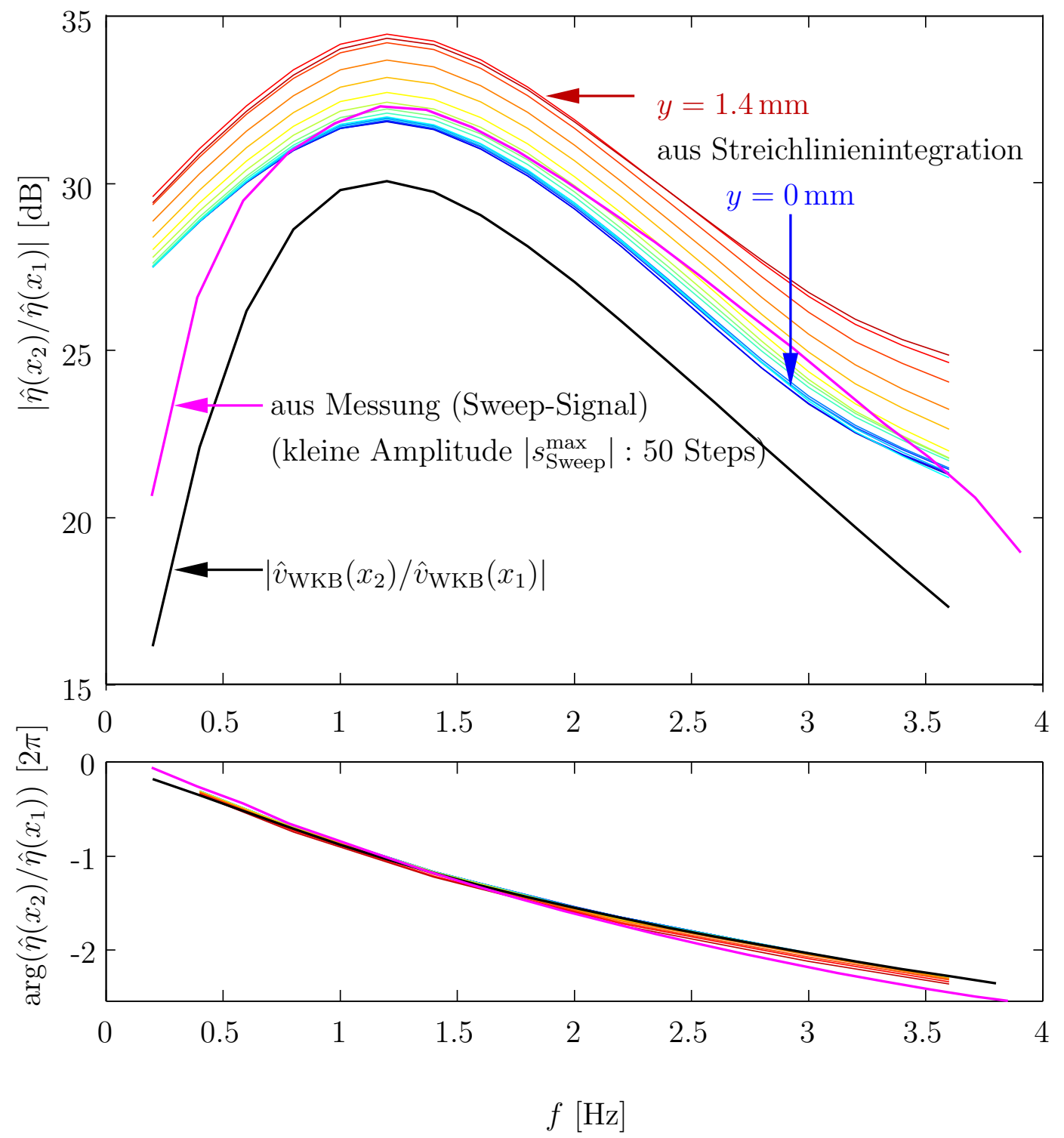

Abb. B.5: Übertragungsfunktion der Strahlinstabilität $\hat{\boldsymbol{\eta}}\left(\boldsymbol{x}_{\boldsymbol{2}}, \boldsymbol{f}\right) / \hat{\boldsymbol{\eta}}\left(\boldsymbol{x}_{\boldsymbol{1}}, \boldsymbol{f}\right), x_{1}=2 d=$ $8 \mathrm{~mm}, x_{2}=7.5 d=30 \mathrm{~mm}$ für verschiedene laterale mittlere Streichlinienpositionen $y$ (von blau zu rot von $y=0 \mathrm{~mm}$ (Strahlmitte) zu $y=1.4 \mathrm{~mm}$ in $0.1 \mathrm{~mm}$ Schritten). 


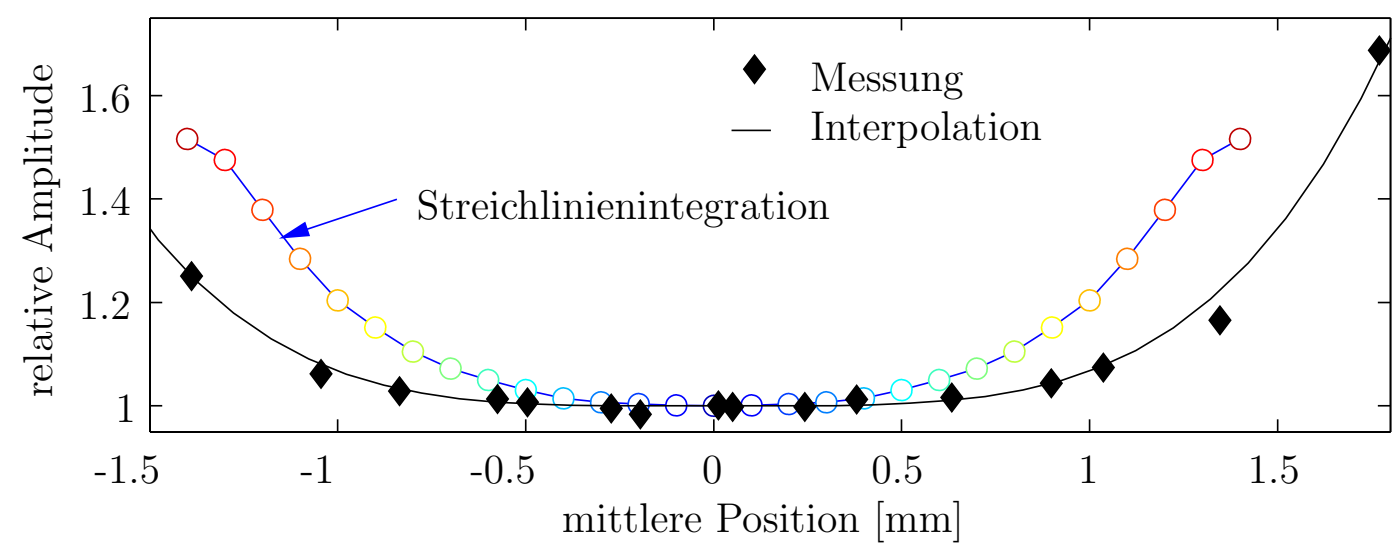

Abb. B.6: Verstärkungsfaktor $\left|\hat{\eta}\left(x_{2}, f\right) / \hat{\eta}\left(x_{1}, f\right)\right|_{y} /\left|\hat{\eta}\left(x_{2}, f\right) / \hat{\eta}\left(x_{1}, f\right)\right|_{y=0}$ für verschiedene mittlere Streichlinienpositionen bezogen auf die Streichlinie in Kanalmitte.

mitte gezeigt. Die Abweichungen sind gerade im tieffrequenten Bereich relativ groß, zum Vergleich ist die Übertragungsfunktion für $\hat{v}_{\mathrm{WKB}}(x)$ dargestellt, deren Übertragungsfaktor deutlich kleiner ist.

Der Übertragungsfaktor wird mit steigendem Abstand $y$ der Streichlinie von der Strahlmitte größer. Vergleicht man dies mit Messergebnissen, so stellt man qualitativ ähnliches Verhalten fest (siehe Abb. B.6). Durch Verschiebung des Farbstoffinjektionspunktes kann die mittlere Streichlinienposition gezielt verändert werden. In der Auswertung wird die mittlere Position der Streichlinie bestimmt und es werden nur solche Sweeps berücksichtigt, deren $y$-Position sich während des Sweeps nur wenig verschoben hat. Die so bestimmte $y$-Abhängigkeit des Übertragungsfaktors kann, da die Phase sehr wenig von der $y$-Position abhängt, in das Messprogramm integriert werden. Allerdings ist die dadurch erzielte Verbesserung der Stabilisierung nicht deutlich nachweisbar, da der Effekt nur sehr klein ist. 



\section{Anhang C}

\section{Rezeptivität}

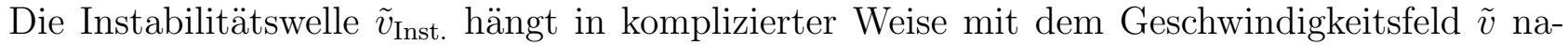
he der Düse zusammen. Dieses hängt wiederum von der Wechselwirkung des anregenden Potenzialfelds $\left.\hat{v}\right|_{\bar{U}=0}$ mit der Gleichströmung in Düsennähe ab. Dabei kann man sich $\left.\hat{v}\right|_{\bar{U}=0}$ auf verschiedene Weisen erzeugt denken z.B. durch das Druckfeld $\left.\left.\nabla \tilde{p}\right|_{\bar{U}=0}\right|_{\bar{U}=0}$ des Aktuators oder durch das Druckfeld der Strahl-Kanten-Wechselwirkung. Die Umsetzung von $\left.\hat{v}\right|_{\bar{U}=0}$ in die Instabilitätswelle lässt sich also in zwei Teilprozesse zerlegen, die miteinander wechselwirken. Hier sollen diese Prozesse in einem sehr stark vereinfachten Bild durch zwei hintereinandergeschaltete Übertragungsfunktionen charakterisiert sein (siehe Abb. C.1).

Das Druckfeld bzw. das Geschwindigkeitsfeld des Aktuators wird bei ruhender Gleichströmung in ein Geschwindigkeitsfeld umgesetzt, das sich aufgrund der Strömungsüberlagerung stark von dem ohne Gleichströmung unterscheidet (Strahlrezeptivität). Dieses Wechselgeschwindigkeitsfeld regt die Instabilitätswelle an.

Die Streichlinie integriert über dieses modellhaft zusammengesetzte Feld. Eine Rückrechnung der Streichlinienauslenkung in die einzelnen Anteile wird in Abschnitt C.4 diskutiert.

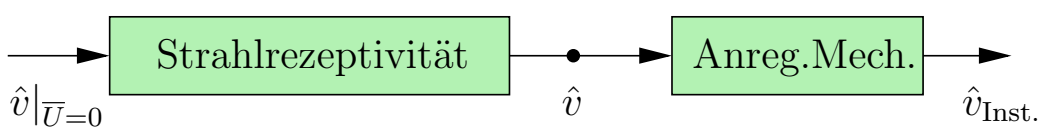

Abb. C.1: Übertragungsfunktion der Instabilitätswellenanregung in zwei Teilprozesse zerlegt. 


\begin{tabular}{|c|c|c|c|c|c|}
\hline $\begin{array}{c}\text { Encoder- } \\
\text { impulse } \\
{[\text { Step }]}\end{array}$ & Volumen & \multicolumn{2}{|c|}{ max. Auslenkung $\zeta_{\text {Akt. }}^{\mathrm{n}}$} & \multicolumn{2}{|c|}{ x-Position des Maximums } \\
\hline $\left.\mathrm{mm}^{3}\right]$ & $\mathrm{d}=4 \mathrm{~mm}$ & $\mathrm{~d}=2 \mathrm{~mm}$ & $\mathrm{~d}=4 \mathrm{~mm}$ & $\mathrm{~d}=2 \mathrm{~mm}$ \\
{$[\mu \mathrm{m}]$} & {$[\mu \mathrm{m}]$} & {$[\mathrm{mm}]$} & {$[\mathrm{mm}]$} \\
\hline 1 & 0.1885 & 0.141 & 0.182 & 1.6 & 0.8 \\
\hline
\end{tabular}

Tabelle C.1: Umrechnung der Aktuatoramplitude in die Potenzialfeldauslenkung.

\section{C.1 Normierung der Streichlinienauslenkung auf die Auslenkung des Potenzialfeldes}

Zur Normierung der Streichlinienauslenkung wird die Auslenkungsamplitude $\hat{\zeta}_{\text {Akt. }}$ des Potenzialfeldes der Aktuatoranregung herangezogen. Das Anregungsfeld des Aktuators lässt sich reibungsfrei durch ein komplexes Potenzial beschreiben, und die Akutator-Membranen können durch Punktquellen dargestellt werden. Das gesuchte Strömungsfeld kann durch die SchwarzChristoffel-Transformation berechnet werden. Dazu muss eine Halbebene (Kanal ohne Keil) oder ein Streifen (Kanal mit Keil [21]) der komplexen Ebene auf das Kanalinnere abgebildet werden. Mittels der MATlaB Schwarz-Christoffel-Toolbox von Tobi Driscoll [11] kann diese Abbildung für den hier verwendeten Kanal berechnet werden. Details finden sich bei Preckel 1999 [39] .

Die Quellstärke pro Schrittmotorschritt, also pro Step, kann aus der Pumpengeometrie entnommen werden und ist in Tabelle C.1 aufgelistet.

Als Normierung $\zeta_{\text {Akt. }}^{\mathrm{n}}$ wird die maximale Potenzialfeldauslenkung (Abb. C.2) in Kanalmitte $y=0$ ohne Anwesenheit des Keils herangezogen:

$$
\zeta_{\text {Akt. }}^{\mathrm{n}}=\left|\hat{\zeta}_{\text {Akt. }}\left(x_{\max }, 0\right)\right| ; \operatorname{mit} x_{\max } \in[0, \infty]:\left|\hat{\zeta}_{\text {Akt. }}\left(x_{\max }, 0\right)\right|=\max
$$

und für die Normierung $v_{\text {Akt. }}^{\mathrm{n}}$ der Wechselgeschwindigkeiten entsprechend $v_{\text {Akt. }}^{\mathrm{n}}=\left|\hat{v}_{\text {Akt }}\left(x_{\max }, 0\right)\right|$. Die Normierung ist damit abhängig von der verwendeten Düsenbreite.

\section{C.2 Strahlrezeptivität (Störungsannahme des Frei- strahls)}

Im Folgenden wird die Umsetzung des Aktuatorfelds in das Geschwindigkeitsfeld in Düsennähe in Abhängigkeit von der Aktuatorfrequenz und der Gleichströmungsgeschwindigkeit in der Düse untersucht. Dabei ist das Aktuatorfeld das Potenzialfeld bei im Mittel ruhendem Medium. Dem Freistrahl wird beim Verlassen der Düse eine laterale, lokal konzentrierte Störung in Form des durch den Aktuator erzeugten Druckgradienten aufgeprägt. Dieses Druckfeld des Aktuators führt in Abhängigkeit von der Freistrahlgeschwindigkeit und der Störfrequenz zu einer lateralen 

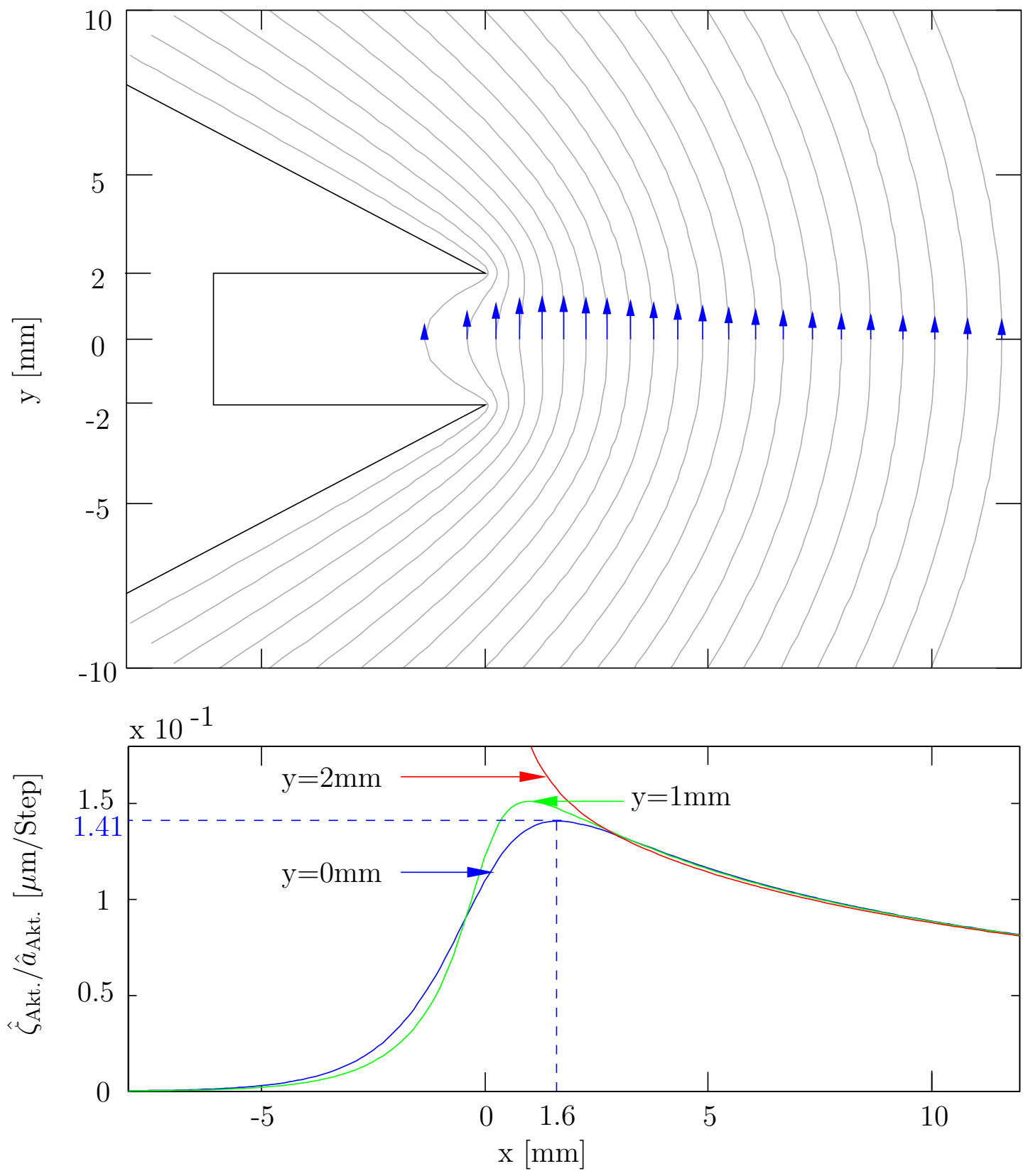

Abb. C.2: Potenzialströmung. Oben: Stromlinien, unten: Auslenkung für die Schnitte $\mathrm{y}=0 \mathrm{~mm}, \mathrm{y}=1 \mathrm{~mm}$ und $\mathrm{y}=2 \mathrm{~mm}$. 
Auslenkung der Fluidelemente. Das Druckfeld der Störung selbst ist allerdings auch von diesen Größen in einer schwer beschreibbaren Weise abhängig, da das auf diese Weise berechnete Störfeld nicht die Kontinuitätsgleichung und die Randbedingungen erfüllt. Um alle Faktoren zu berücksichtigen, muss man die Navier-Stokes-Gleichungen lösen. Im folgenden Modell soll der rückwirkende Anteil des Druckfelds der Störung, der zur Erfüllung der Kontinuitäts-Gleichung und der Randbedingung notwendig ist, vernachlässigt werden.

\section{C.2.1 Zum Einfluss der Strömung auf die laterale Geschwindigkeit der Teilchen}

Hier wird nur der Einfluss der Konvektion durch das Druckgradientenfeld betrachtet, also $\frac{\tilde{v} \mid \bar{U} \neq 0}{\left.\tilde{v}\right|_{\bar{U}=0}}$, wobei hier $\left.\tilde{v}\right|_{\bar{U}=0}=\tilde{v}_{\text {Akt. }}$ das Potenzialfeld des Aktuators sein soll. Im Gegensatz zum Fall ohne Gleichströmung wird das Teilchen nur während der Zeit beschleunigt, während der es sich im Nahfeld der Düse aufhält, dessen Ausdehnung mit der Düsenbreite $d$ skaliert. Die zu verschiedenen Phasen ausgeübten Kräfte der Schwingung gehören zu verschiedenen Stellen des Nahfeldes, haben also unterschiedliche Amplituden. Nur wenn ein Teilchen während einer Schwingungsperiode praktisch am gleichen Punkt des Nahfeldes bleibt $(U / d) / f \ll 1$ (große Strouhalzahl) hat die Konvektion keinen nennenswerten Einfluss, daher ist dann das Amplitudenverhältnis

$$
\lim _{\operatorname{Sr}_{\mathrm{d}} \rightarrow \infty} \frac{\left.\hat{v}\right|_{\bar{U} \neq 0}}{\left.\hat{v}\right|_{\bar{U}=0}}=1 .
$$

Im umgekehrten Grenzfall (kleine Strouhalzahl) wird das Teilchen nur während eines Ausschnittes der Schwingungsperiode beschleunigt und

$$
\lim _{\mathrm{Sr}_{\mathrm{d}} \rightarrow 0} \frac{\left.\hat{v}\right|_{\bar{U} \neq 0}}{\left.\hat{v}\right|_{\bar{U}=0}}=0 .
$$

Die Übertragungsfunktion zwischen $\left.\tilde{v}\right|_{\bar{U}=0}$ und $\tilde{v}(x)=\left.\tilde{v}(x)\right|_{\bar{U} \neq 0}$ führt also zu einer Hochpasscharakteristik bezüglich der Strouhalzahl. Dies soll im Folgenden durch eine Rechnung ausgeführt werden.

Betrachtet man das Fluidelement, das zur Zeit $t$ den Ort $x$ erreicht hat, so hat das Fluidelement zu den vergangenen Zeiten $t^{\prime}$ die Orte

$$
x^{\prime}\left(t^{\prime}\right)=x-\left(t-t^{\prime}\right) \bar{U}
$$

durchlaufen. Das Fluidelement unterliegt dort dem lateralen Druckgradienten des anregenden Feldes $\left.\nabla \hat{p}\left(x\left(t^{\prime}\right)\right)\right|_{\bar{U}=0} \cdot e^{i \omega t^{\prime}}$. Die laterale Wechselgeschwindigkeit mit Gleichströmung $\tilde{v}(x, t)$ ergibt sich dann zu:

$$
\tilde{v}\left(x, x_{\text {start }}, t\right)=-\left.\frac{1}{\rho} \int_{t-\left(\frac{x-x_{\text {start }}}{\bar{U}}\right)}^{t} \nabla \hat{p}\left(x\left(t^{\prime}\right)\right)\right|_{\bar{U}=0} e^{i \omega t^{\prime}} d t^{\prime} .
$$


Der Ort der Teilcheninjektion ist mit $x_{\text {start }}$ symbolisiert. Innerhalb der Düse mit der Breite $d$ wächst das Potenzialfeld exponentiell an, so dass man in unmittelbarer Düsennähe

$$
\left.\left.\nabla \hat{p}\left(x\left(t^{\prime}\right)\right)\right|_{\bar{U}=0} \approx \nabla \hat{p}^{0}\right|_{\bar{U}=0} e^{\sigma x\left(t^{\prime}\right)} \text { mit } \sigma=\pi / d
$$

approximieren kann. Diese ist die am weitesten in den Düsenkanal hineinreichende Mode. Damit kann nun die Gleichung (C.5) integriert werden:

$$
\begin{aligned}
\tilde{v}\left(x, x_{\text {start }}, t\right) & =-\left.\frac{1}{\rho} \int_{t-\left(\frac{x-x_{\text {start }}}{\bar{U}}\right)}^{t} \nabla \hat{p}^{0}\right|_{\bar{U}=0} e^{\sigma\left(x-\bar{U}\left(t-t^{\prime}\right)\right)} e^{i \omega t^{\prime}} d t^{\prime} \\
& =-\frac{1}{\rho} \frac{\left.\nabla \hat{p}^{0}\right|_{\bar{U}=0} e^{\sigma x}}{\sigma \bar{U}+i \omega}\left[1-e^{\left.-(\sigma \bar{U}+i \omega)\left(\frac{x-x_{\text {start }}}{\bar{U}}\right)\right] \cdot e^{i \omega t} .}\right.
\end{aligned}
$$

Denkt man sich die Teilcheninjektion bei $x_{\text {start }}=-\infty$, so erhält man:

$$
\tilde{v}(x, t)=-\frac{1}{\rho} \frac{\left.\nabla \hat{p}^{0}\right|_{\bar{U}=0} e^{\sigma x}}{\sigma \bar{U}+i \omega} e^{i \omega t} .
$$

Mit $-\left.\nabla \tilde{p}(x)\right|_{\bar{U}=0}=\left.i \omega \rho \tilde{v}(x)\right|_{\bar{U}=0}=\left.\hat{v}^{0}\right|_{\bar{U}=0} e^{\sigma x}$ und durch Fouriertransformation ergibt sich als Aktuatorübertragungsfunktion von der Aktuatorschnelle $\left.\tilde{v}\right|_{\bar{U}=0}$ auf die laterale Querschnelle $\tilde{v}$ :

$$
\frac{\hat{v}(x, \omega)}{\left.\hat{v}^{0}\right|_{\bar{U}=0}}=\frac{e^{\sigma x}}{1+\frac{\sigma \bar{U}}{i \omega}}
$$

ein Hochpass erster Ordnung.

Um die messbare Streichlinienauslenkung in Düsennähe auszurechnen, kann man prinzipiell genauso vorgehen. Die Streichlinie ist als Bahn aller Teilchen, die vom Ort $x_{\text {start }}=-\infty$ ausgehen, gegeben durch:

$$
\tilde{\eta}\left(x, x_{\text {start }}, t\right)=\int_{-\infty}^{t} \hat{v}\left(x\left(t^{\prime}\right)\right) e^{i \omega t^{\prime}} d t^{\prime} .
$$

Setzt man nun für $\hat{v}(x)$ das durch die Übertragungsfunktion in Gl. (C.9) gegebene Feld bezogen auf die vom Aktuator erzeugte Wechselgeschwindigkeit ein, so erhält man nach identischer Rechnung mit $\left.\tilde{v}\right|_{\bar{U}=0}=\left.i \omega \tilde{\zeta}\right|_{\bar{U}=0}$ :

$$
\begin{aligned}
\tilde{\eta}(x, t) & =\int_{-\infty}^{t} \frac{\left.\hat{v}^{0}\right|_{\bar{U}=0} e^{\sigma x(t)} e^{i \omega t^{\prime}}}{1+\frac{\sigma \bar{U}}{i \omega}} d t^{\prime} \\
& =\frac{\left.\tilde{\zeta}^{0}\right|_{\bar{U}=0} e^{\sigma x}}{\left(1+\frac{\sigma \bar{U}}{i \omega}\right)^{2}} e^{i \omega t} .
\end{aligned}
$$

Daraus ergibt sich für den gesamten Prozess eine Übertragungscharakteristik, die einem Hochpass zweiter Ordnung entspricht:

$$
\frac{\hat{\eta}(x, \omega)}{\left.\tilde{\zeta}^{0}\right|_{\bar{U}=0}}=e^{\sigma x} \frac{\left(\frac{i \omega}{\sigma \bar{U}}\right)^{2}}{\left(1+\frac{i \omega}{\sigma \bar{U}}\right)^{2}} .
$$



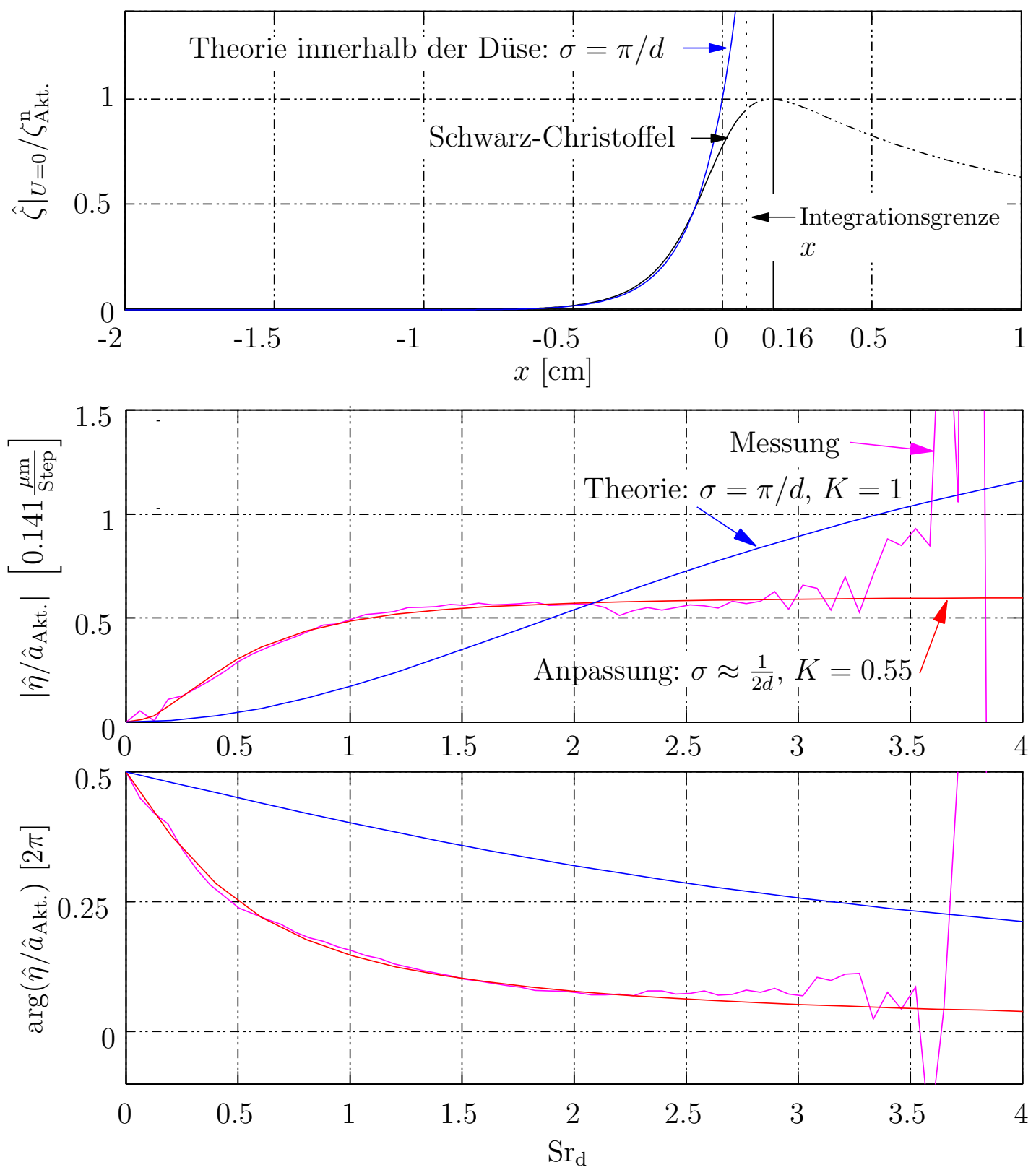

Abb. C.3: Streichlinienauslenkung/Aktuatoramplitude normiert auf $\zeta_{\text {Akt. }}^{\mathrm{n}}=1.41 \cdot 10^{-1} \mu \mathrm{m}$ in Abhängigkeit von der Strouhalzahl. Die Normierung ist auf das Maximum des Potenzialfeldes bezogen, das durch die Schwarz-Christoffel-Transformation bestimmt wurde (oben). 


\begin{tabular}{|l|c|c|c||c||c|}
\hline & \multicolumn{3}{|c||}{ Anpassung von 3 Parametern } & \multicolumn{2}{c|}{ doppelter Hochpass } \\
& HP I & HP II & TP I & HP I+II Mess. & HP I+II Theorie \\
\hline Eckfrequenz $[\mathrm{Hz}]$ & 0.76 & 3.2 & 24.5 & 1.55 & 9.75 \\
Eckfrequenz $\mathrm{Sr}_{\mathrm{d}}$ & 0.245 & 1.03 & 7.9 & 0.5 & $\pi$ \\
\hline
\end{tabular}

Tabelle C.2: Eckfrequenzen für die Parameterisierung der Aktuatorübertragungsfunktion

Preckel 1999 [39] hatte experimentell bei der Durchflussgeschwindigkeit $\bar{U}=5.36 \mathrm{~cm} / \mathrm{s}$ beobachtet, dass sich die Übertragungsfunktion von der Potenzialfeldauslenkung $\left|\hat{\zeta}\left(x_{\max }\right)\right|_{U=0}=\zeta_{\text {Akt. }}^{\mathrm{n}}$. am Ort des Maximums (siehe Abb. C.3 (oben)) zur Streichlinienauslenkung direkt unterhalb der Düse durch ein Filter beschreiben lässt, das sich aus zwei unabhängigen Hochpässen (HP) und einem Tiefpass (TP) mit einem Verstärkungsfaktor $K$ zusammensetzt:

$$
\frac{\hat{\eta}(x, \omega)}{\left.\hat{\zeta}\left(x_{\max }\right)\right|_{U=0}}=K \frac{\frac{i \omega}{\omega_{\mathrm{HP} 1}}}{1+\frac{i \omega}{\omega_{\mathrm{HP} 1}}} \frac{\frac{i \omega}{\omega_{\mathrm{HP} 2}}}{1+\frac{i \omega}{\omega_{\mathrm{HP} 2}}} \frac{1}{1+\frac{i \omega}{\omega_{\mathrm{TP} 1}}} .
$$

Die gefundene Tiefpasscharakteristik ist dabei lediglich durch die Hysterese des Aktuators entstanden, da die Auslenkungen, die zur Messung höherer Frequenzen verwendet werden, klein sind (siehe Abschnitt 2.4). Die Parameter sind in Tabelle C.2 aufgeführt. Dabei wurde die Strouhal-Zahl $\mathrm{Sr}_{\mathrm{d}}=2 \pi \mathrm{fd} / \mathrm{U}_{0}$ auf die Maximalgeschwindigkeit $U_{0}$ am Ausfluss bezogen $\left(U_{0}=7.8 \mathrm{~cm} / \mathrm{s}, d=0.4 \mathrm{~cm}\right)$.

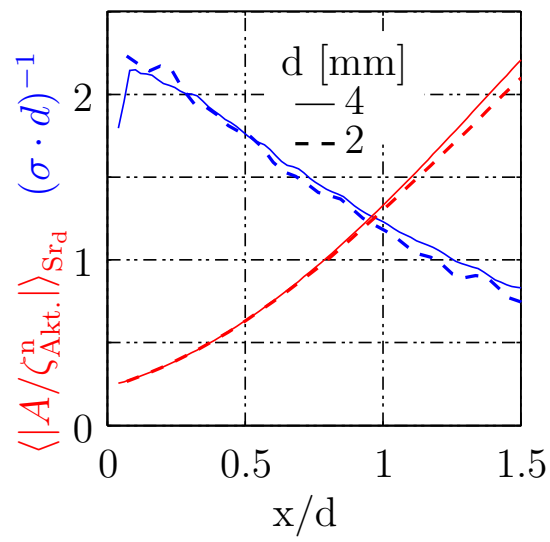

Abb. C.4: Mittlerer Übertragungs-

faktor für $\mathrm{Sr}_{\mathrm{d}}<1$ bezogen auf die Maximalauslenkung des Potenzialfeldes.
Auf Grund des stark vereinfachenden Modells und der in Abb. C.3 gezeigten Abweichung zwischen dem im Ansatz verwendeten Feld $\left.\hat{\zeta}_{\text {Akt. }}^{0}\right|_{U=0} e^{\sigma x}$ und dem theoretischen durch die Schwarz-Christoffel-Transformation berechneten Feld, kann eine Übereinstimmung der Gl. (C.12) mit den Messergebnissen nicht erwartet werden.

Das theoretisch berechnete Modell der Rezeptivität führt $\mathrm{zu}$ einem Hochpass zweiter Ordnung mit einer theoretischen Eckfrequenz $\left(\sigma U_{0}=\pi / d \cdot U_{0}\right)$ von $\mathrm{Sr}_{\mathrm{dHP} 1 / 2}=\pi$. Die beste Anpassung an alle Übertragungsfunktionen - für verschiedene Strömungsgeschwindigkeiten und Anregungsfrequenzen - erhält man für $\sigma=1 /(2 d)$ und damit für $\operatorname{Sr}_{\mathrm{dHP} 1 / 2}=0.5$ und $K=0.5$. Die hier bestimmten Eckfrequenzen entsprechen etwa dem Mittel der Zeitkonstanten, die von PRECKEL angegeben wurden (siehe zusammenfassend Tab. C.2).

In Abb. C.3 ist eine experimentelle Übertragungsfunktion mit dem hier gemachten Modell 
verglichen. Das durch die Übertagungsfunktion angepasste $\sigma$ des Potenzialfelds ist etwa eine Größenordnung kleiner als $\pi / d$. Betrachtet man aber die Form der Übertragungsfunktion $A$ stromab, so stellt man in den Frequenzbereichen, in denen der Einfluss der Instabilitätswelle vernachlässigbar ist, eine sehr gute Übereinstimmung mit einem Hochpass zweiter Ordnung fest.

Das Anwachsen des mittleren Übertragungsfaktors $\left\langle\hat{\eta}(x, \omega) /\left.\hat{\zeta}\left(x_{\max }\right)\right|_{U=0}\right\rangle_{\omega}$ und auch des optimal angepassten $\sigma$ (siehe Abb. C.4) mit dem Laufweg $x$ ist in Düsennähe für verschiedene Strömungsgeschwindigkeiten, Düsenbreiten und Störfrequenzen erstaunlicherweise sehr ähnlich. Daher kann man für dieses Experiment die universelle Übertragungsfunktion der Strahlrezeptivität mit den Parametern $\sigma d=1 / 2$ und $K \approx 0.5$ nach Gleichung (C.12) für einen Ort $x_{\mathrm{D}}$ sehr nahe der Düsenöffnung durch

$$
\frac{\hat{\eta}\left(x_{\mathrm{D}}, \mathrm{Sr}_{\mathrm{d}}\right)}{\left.\hat{\zeta}\left(x_{\max }\right)\right|_{U=0}} \approx \frac{1}{2} e^{x_{\mathrm{D}} /(2 d)} \frac{\left(2 \cdot \mathrm{Sr}_{\mathrm{d}} \cdot \mathrm{i}\right)^{2}}{\left(1+2 \cdot \mathrm{Sr}_{\mathrm{d}} \cdot \mathrm{i}\right)^{2}}
$$

angeben.

\section{C.3 Anregung der Instabilitätswelle}

Das vom Aktuator induzierte Geschwindigkeitsfeld $\tilde{v}$ ist über etwa 1.5 Düsenbreiten stromab ausgedehnt. In diesem Bereich findet die Anregung der Instabilitätswelle statt. Betrachtet man den Keim der Instabilitätswelle als einen Anteil an diesem Feld, so muss für diesen Feldanteil die gleiche Beziehung zwischen den für die Ausbreitung charakteristischen Größen gelten wie bei der Instabilitätswelle selbst. Die Frage ist, in welcher Beziehung der Strömungsgrößen $\tilde{p}, \tilde{u}, \tilde{v}, \nabla_{\mathrm{x}} \tilde{u}, \nabla_{\mathrm{x}} \tilde{v}, \ldots$ sich der Instabilitätsmechanismus in größter Klarheit widerspiegelt. Es ist anzunehmen, dass diese Beziehung auch vom Gleichströmungsprofil abhängig ist. Für die hier betrachteten zwei Moden der gedämpften und der angefachten Freistrahlinstabilitätswelle sollte diese Beziehung möglichst so sein, dass sich die beiden Moden klar voneinander separieren lassen.

Experimentell lassen sich mit dem hier verwendeten Aufbau die Größen $\tilde{v}, \nabla_{\mathrm{x}} \tilde{v}, \ldots$ nicht einzeln ansteuern, so dass es nicht möglich ist, eine Aussage darüber zu treffen, welche Beziehung den Anregungsmechanismus kennzeichnet. Man kann aber experimentell eine Aussage zu der Phasenbeziehung zwischen Aktuator und Instabilitätswelle treffen. Dazu wird das WKBGeschwindigkeitswechselfeld herangezogen und die jeweils zugehörige Streichlinie integriert, die Anfangsparameter der WKB-Lösung an die Messergebnisse im Fernfeld angepasst und dann auf die Düsenposition zurückextrapoliert. Die Amplitude der Streichlinienauslenkung $\tilde{\eta}$ hängt besonders stark von nichtlinearen Effekten bei der Anregung und Ausbreitung ab. Daher beschränke ich mich auf die Phasenbeziehung zwischen der Auslenkung des Poentenzialfeldes am Ort des Maximums $\left.\hat{\zeta}\left(x_{\max }\right)\right|_{U=0}$ und der auf den Düsenort $x=0.08 \mathrm{~mm}$ zurückinterpolierten Phase der Instabilitätswellenauslenkung $\tilde{\eta}\left(x_{\mathrm{D}}\right)$. Man stellt experimentell fest, dass diese 


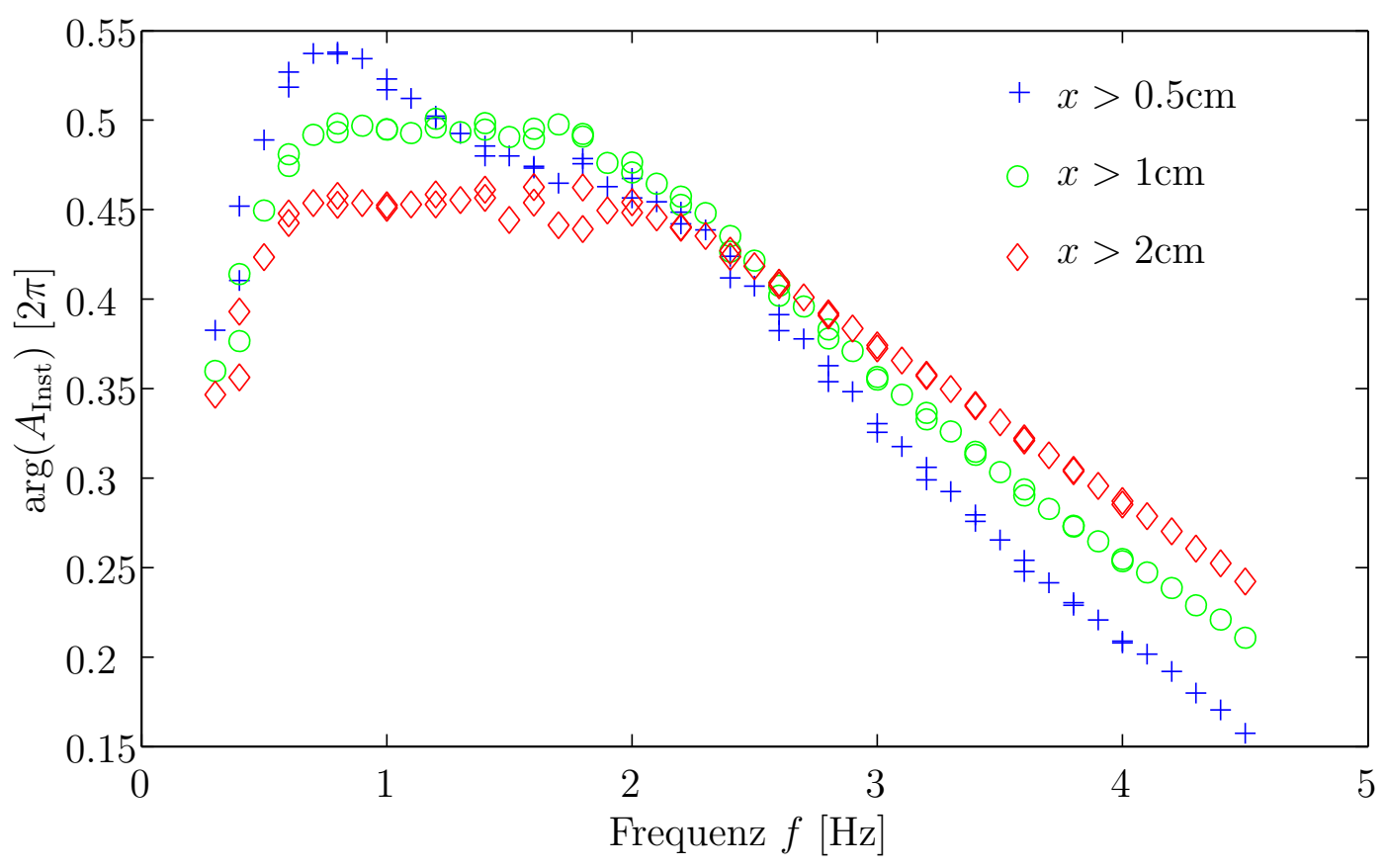

Abb. C.5: Anregung der Instabilitätswelle. Extrapolierte Phase zum Ort $x=0.08 \mathrm{~mm}$, $U_{\mathrm{D}}=5.36 \mathrm{~cm} \cdot \mathrm{s}^{-1}$. Als Parameter ist der Ort angegeben, ab dem die Daten zur Extrapolation auf den Düsenort benutzt wurden. Mit steigender Entfernung von der Düse wird die Steigung $\left|\frac{d \arg \hat{\eta}}{d x}\right|$ größer, somit ergibt sich eine größere Phasendrehung bei der Extrapolation.

Phase gegen die Phase des Aktuators um etwa $\pi$ verschoben ist, wie in der Auftragung von $A_{\text {Inst }}=\hat{\eta}_{\text {Inst. }}\left(x_{\mathrm{D}}\right) /\left.\hat{\zeta}\left(x_{\max }\right)\right|_{U=0}$ in Abb. C.5 zu sehen ist.

\section{C.4 Bereinigung der Streichlinienauslenkung}

Die gemessene Streichlinienauslenkung $\tilde{\eta}$ entsteht durch Integration über das Geschwindigkeitsfeld

$$
\underline{U}(x, y, t)=\underline{\bar{U}}+\underline{\tilde{u}}_{\text {Inst. }}+\underline{\tilde{u}}_{\mathrm{Akt}},
$$

wobei das Instabilitätswellenfeld $\underline{\tilde{u}}_{\text {Inst. }}$ und das ohne Strömungsüberlagerung definierte Aktuatorfeld $\underline{\tilde{u}}_{\text {Akt. }}$ über das Gleichströmungsprofil miteinander wechselwirken, so dass Gl. (C.15) nur ein Ansatz für das Strömungsfeld darstellt.

Im Folgenden soll versucht werden, die gemessene Streichlinienauslenkung in einen Anteil $\tilde{\eta}_{\text {Akt. }}$ aufgrund des anregenden Aktuatorfeldes und in einen Anteil $\tilde{\eta}_{\text {Inst. }}$ aufgrund des Instabilitäts- 
wellenfeldes zu zerlegen:

$$
\hat{\eta}=\hat{\eta}_{\text {Inst. }}+\hat{\eta}_{\text {Akt. }} .
$$

Dazu werden zunächst die Streichlinien untersucht, die aus den einzelnen Wechselfeldern und dem superponierten Wechselfeld entstehen.

- Die Streichlinie $\tilde{\eta}_{\text {Akt. }}$, die aus dem Potenzialfeld resultiert, wird durch das laterale Geschwindigkeitsfeld

$$
\tilde{v}_{\mathrm{Akt}}(x, y)=\hat{v}_{\mathrm{Akt}}(x, y \equiv 0) e^{i \omega_{\mathrm{s}} t}=\hat{\zeta}_{\mathrm{Akt} .}(x, y \equiv 0) \cdot i \omega e^{i \omega_{\mathrm{s}} t}
$$

$\left(\hat{\zeta}_{\text {Akt. }}(x, y \equiv 0)\right.$ aus Abb. C.2) integriert.

- Die Streichlinie $\tilde{\eta}_{\text {Inst. }}$, die aus dem Instabilitätswellenfeld resultiert, wird durch das laterale Geschwindigkeitsfeld

$$
\tilde{v}_{\text {Inst. }}(x, y)=A_{0} \cdot e^{-i k \cdot x} e^{i \omega_{s} t}
$$

integriert.

- Die Streichlinie $\tilde{\eta}=\tilde{\eta}_{\text {Inst. }}+\tilde{\eta}_{\text {Akt. }}$ wird über das superponierte Feld:

$$
\tilde{v}=\tilde{v}_{\text {Akt }}(x, y)+\tilde{v}_{\text {Inst. }}(x, y)
$$

integriert.

In Abb. C.6 (oben) sind die einzelnen Anteile dargestellt, die man durch die numerische Streichlinienintegration über die entsprechenden $\tilde{v}$-Felder gewinnt, wobei die $\tilde{u}$-Komponenten vernachlässigt werden und $\bar{U}(x, y) \equiv 5 \mathrm{~cm} \cdot \mathrm{s}^{-1}, \bar{V}(x, y) \equiv 0$.

Für die Streichlinie $\hat{\eta}_{\text {Akt. }}$, die nur aus dem Aktuatorfeld ohne Instabilitätswelle resultiert, entsteht nach dem Maximum von $\left|\tilde{v}_{\text {Akt }}\right|$ (roter Pfeil) eine Welle (gestrichelte Linien kennzeichnen der Real und Imaginärteil von $\left.\hat{\eta}_{\text {Akt. }}\right)$. Betrachtet man die Beträge, so wächst $\left|\hat{v}_{\text {Inst. }}\right|$ erwartungsgemäß ungefähr exponentiell an, während die Beträge von $\left|\hat{\eta}_{\text {Akt. }}\right|$ und $|\hat{\eta}|$ eine gewisse Welligkeit aufweisen, die durch das Aktuatorfeld verursacht wird, da das gesamte Strömungsfeld durch $\tilde{v}$ gleichphasig verschoben wird.

Die Welligkeit von $|\hat{\eta}|$ soll vermindert werden. Dazu wird zunächst durch Bildung des komplexen Gradienten die Welligkeit noch deutlicher (siehe Abb. C.6 (unten)). Daher kann die zweite Ableitung des komplexen Gradienten als günstiges Maß für die Welligkeit $\left|\frac{\partial^{2} i \nabla_{x} \ln \left(\hat{\eta} / \zeta_{\text {Akt. }}^{\mathrm{n}}\right)}{\partial x^{2}}\right|$ definiert werden. Die Frage ist, ob es eine einfache Möglichkeit gibt, $\hat{\eta}$ so zu bereinigen, dass der

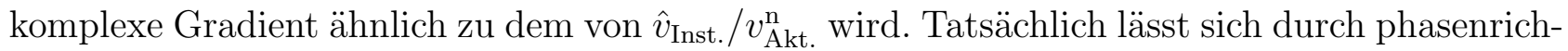
tiges skaliertes Abziehen der Auslenkung $\hat{\zeta}_{\text {Akt. }}(x, y \equiv 0)$ aus der Streichlinie eine Verminderung der Welligkeit erreichen:

$$
\left.\tilde{\eta}_{\text {Inst. }}(x)=\tilde{\eta}(x)-C \tilde{\zeta}_{\text {Akt. }}(x, y \equiv 0)\right) \text { mit } C \in \mathbf{C}
$$



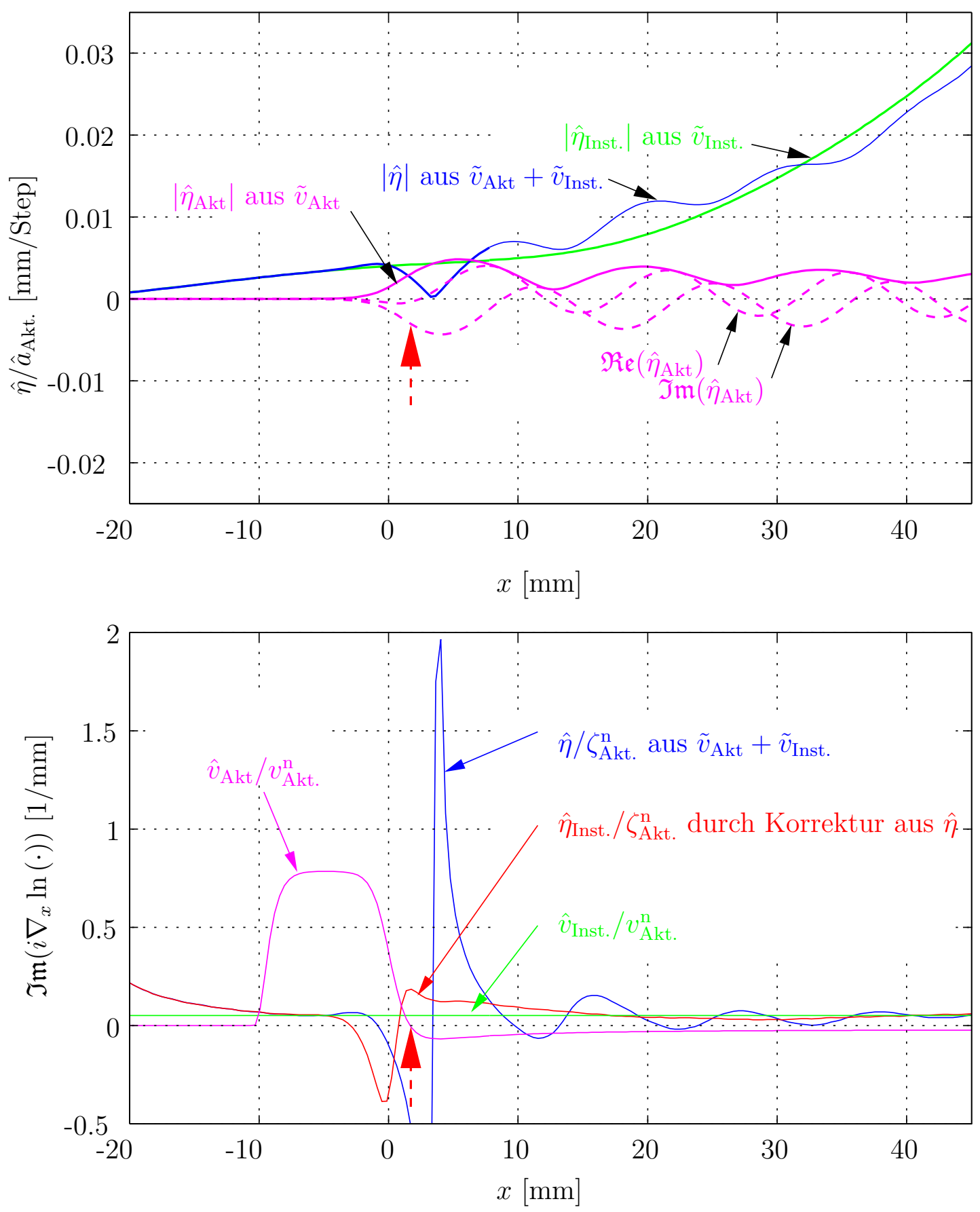

Abb. C.6: Streichlinien auf Basis verschiedener Wechselgeschwindigkeitsfelder. Oben: Vergleich zwischen den Beträgen der Streichlinienauslenkung. Für die Streichlinie, die aus $\tilde{v}_{\text {Akt. }}$ resultiert, ist zusätzlich die sich ausbreitende Welle gezeigt $\left(\mathfrak{R e}\left(\hat{\eta}_{\mathrm{Akt}}\right), \mathfrak{I m}\left(\hat{\eta}_{a} k t\right)\right)$. Unten: komplexer Gradient der unbereinigten Streichlinie $\hat{\eta}$ und der durch Optimierung korrigierten Streichlinie. Zum Vergleich sind die komplexen Gradienten der Wechselfelder eingezeichnet. Parameter: $f_{\mathrm{s}}=3.6 \mathrm{~Hz}, k=(0.6-i 0.05) 1 / \mathrm{mm},\left|\hat{v}_{\text {Inst. }}(0) / \hat{v}_{\text {Akt }}(0)\right|=0.5$. 

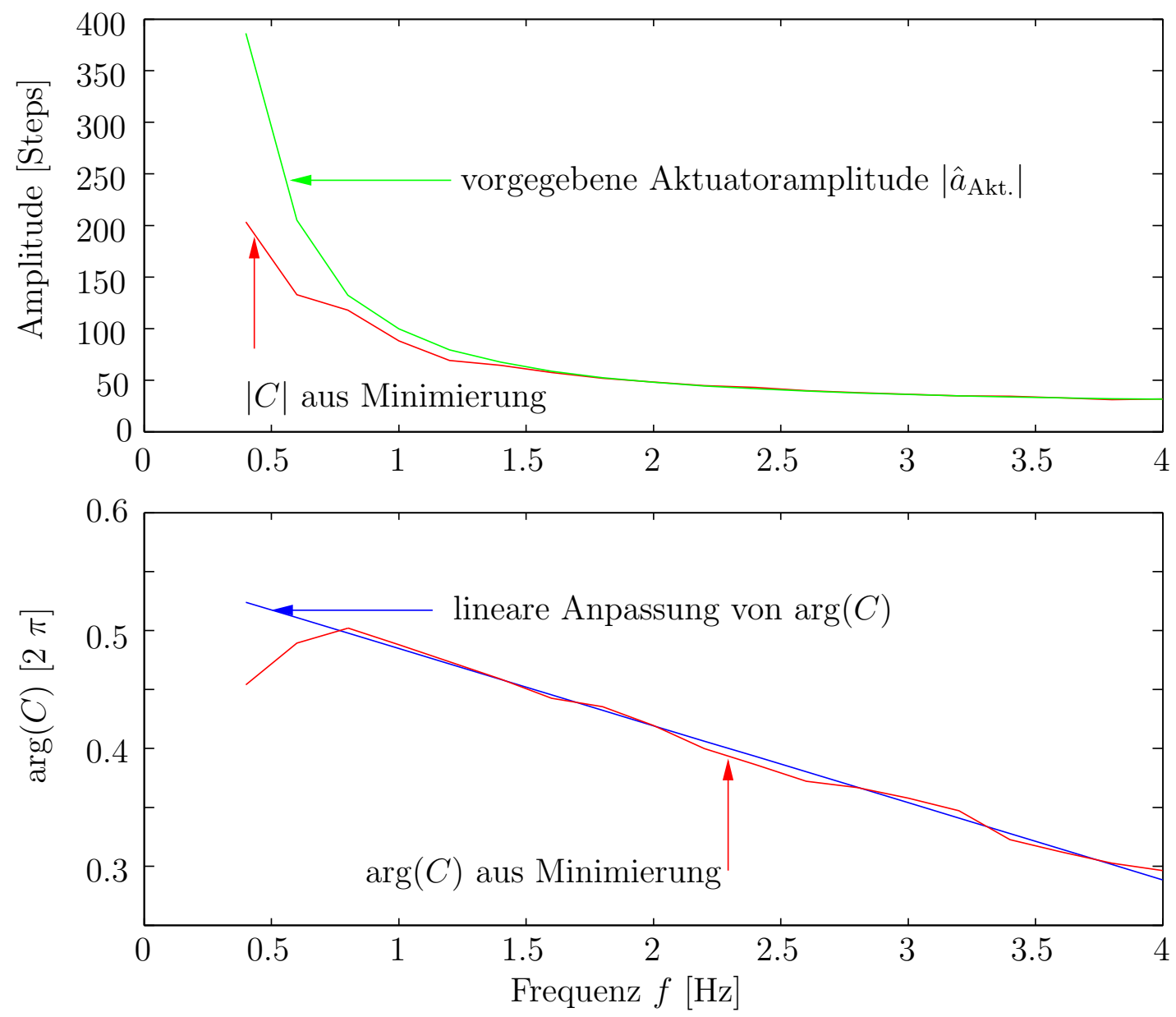

Abb. C.7: Inverse Bestimmung der Amplitude durch Minimierung der Welligkeit des komplexen Gradienten.

und $\arg \{C\}$ ist unabhängig von $x$. Damit lässt sich $C$ durch die Optimierung:

$$
C: \int_{x>2 d}\left|\frac{\partial^{2} i \nabla_{x} \ln \left(\left[\tilde{\eta}(x)-C \tilde{\zeta}_{\text {Akt. }}(x)\right] / \zeta_{\text {Akt. }}^{\mathrm{n}}\right)}{\partial x^{2}}\right| d x=\min
$$

bestimmen. Die komplexen Gradienten in Abbildung C.6 (unten) zeigen, dass die korrigierte Streichlinie im Vergleich zu $\hat{\eta}$ eine deutlich geringere Welligkeit aufweist und etwa die vorgegebene Anfachung des Wechselfeldes trifft.

Optimiert man mit Gl. (C.21) auch experimentell gemessene Streichlinien, so kann sogar die anregende Aktuatoramplitude $\left|\hat{a}_{\text {Akt. }}\right|$ im Nachhinein durch das resultierende $|C|$ bestimmt werden. In Abb. C.7 (oben) zeigt sich eine gute Übereinstimmung der tatsächlich eingestellten Aktuatoramplituden mit denen aus der Optimierung gewonnenen. Die Phase von $C$ fällt erstaunlicherweise etwa linear mit der Frequenz ab, und die Verzögerungszeit $\frac{d \arg (C)}{d \omega}$ ist circa. $65 \mathrm{~ms}$, was etwa der Totzeit des Messsystems von $60 \mathrm{~ms}$ entspricht. 


\section{Anhang D}

\section{D.1 Modellierung der konvektiven Instabilität durch Filterkaskaden mit verschiedenen Nichtlinearitäten}

In Abschnitt 3.7 ist die Modellierung der konvektiven Instabiliät mittels einer Filterkaskade dargestellt. Bezüglich der Simulation des Sättigungsverhaltens der Kaskade kann man verschiedene Ansätze für die Integration der Nichtlinearität in die Filterkaskade wählen. Die betrachteten Nichtlinearitäten müssen eine Sättigung herbeiführen. Daher sind sie von der Form $a \tanh (s(x, t) / a)$ oder polynomial $s(x, t)+\epsilon_{3} s(x, t)^{3}+\epsilon_{5} s(x, t)^{5}+\ldots$.

Zur Modellierung der Nichtlinearität wurden drei Modelle untersucht, die im Folgenden dargestellt werden.

\section{D.1.1 Begrenzung des räumlichen Differenzsignals}

Die Differenz der Streichlinienauslenkung vor und hinter dem Filter $\Delta s$ wird nichtlinear begrenzt und die Anfachung wird somit amplitudenabhängig. Dieses Vorgehen ist auch physikalisch motiviert, da man sich die Verbreiterung des Strömungsprofils als lokalen Geschwindigkeitsgradienten vorstellen kann. Jedoch führt diese Methode zu Laufzeitproblemen, da Eingang und Ausgang laufzeitfrei verbunden sind, und sich daher ein Teil der Information ohne Laufzeit über die gesamte Kette ausbreitet.

Mit einer Nichtlinearität, wie sie in Abb. D.1 (links) dargestellt ist, kann eine qualitativ gute Übereinstimmung mit dem Experiment erreicht werden. Dabei ist der Aufbau so gewählt, dass die Differenz der Signale zwischen zwei Orten, also $s\left(x_{\mathrm{k}+1}\right)-s\left(x_{\mathrm{k}}\right)$, nichtlinear begrenzt wird. Damit wird der räumliche Gradient verkleinert und die Anfachung verringert, solange $\left|\epsilon_{3} s^{2}\right|<1$ ist. Dieses Modell führt bei einem bestimmten $s\left(x_{\mathrm{k}}\right)$ zu einer instantanen Addition von Signalen aller davor liegenden Kaskadenelemente $l$ mit $l<k$.

Damit ist die Signallaufzeit abhängig von der Amplitude des Signals. Die Laufzeit kann noch verändert werden, indem man Verzögerungsglieder einsetzt, die in Abb. D.1 als $z^{-n}$ Symbole (links, graue Symbole) gezeigt sind. Ohne diese zusätzliche Laufzeit steht das Aktuatorsignal 


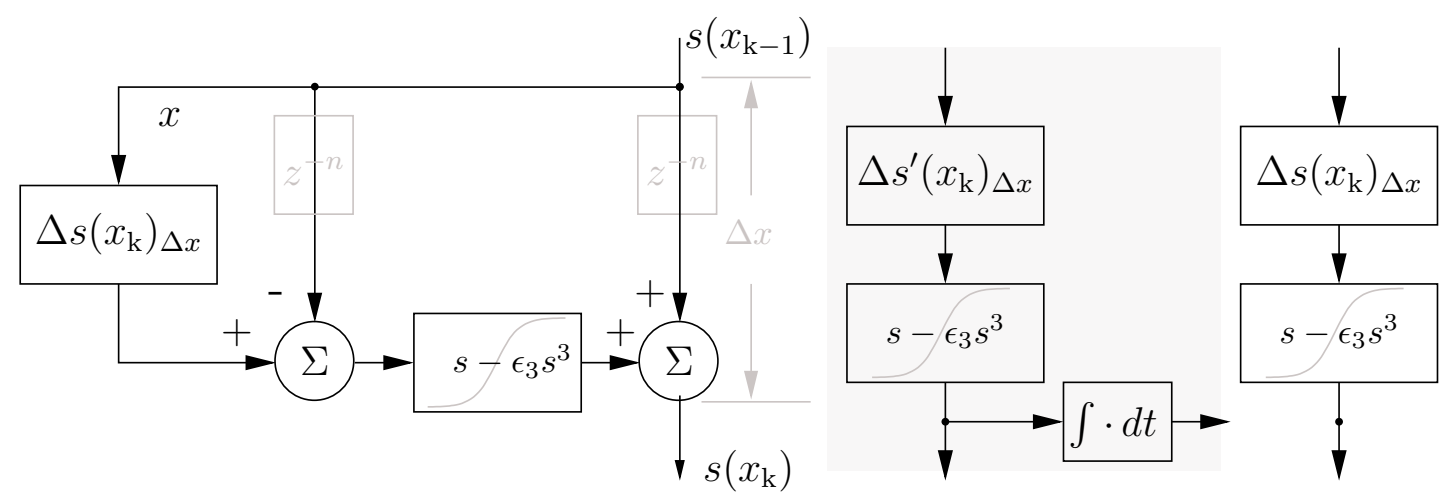

Abb. D.1: Blockdiagramme eines Kaskadenelements. Links: Begrenzung des räumlichen Differenzsignals. Mitte: Begrenzung der zeitlichen Ableitung des Signals. Rechts: Begrenzung des Signals

sofort an jedem Kaskadenelement zu Verfügung, das Modell ist daher nur zur Simulation der konvektiven Instabilität geeignet. Führt man nämlich, wie für die globale Instabilität notwendig, eine Rückkopplung des Signals von der Position $x$ auf die Position $x_{\mathrm{D}}$ ein (siehe Abb. 3.12), so wird das System auf einer falschen Frequenz instabil. Die Nichtlinearität dieses kaskadierten Aufbaus, die durch $\epsilon_{3}$ parametrisiert ist, wurde mit experimentellen Daten solange optimiert, bis die konvektive Instabilität über einen möglichst großen Amplituden- und Frequenzbereich reproduziert werden konnte. Die Ergebnisse für ein Sweepsignal sind in Abb. D.2 mit den experimentellen Daten und dem Modell der Geschwindigkeitsbegrenzung verglichen.

In Abb. 3.16 sind die experimentell gemessenen Auslenkungen und Peaks im Spektrum eines Sinussignals in Abhängigkeit der Amplitude dargestellt, die durch dieses Modell erhalten werden. Man sieht dort, dass die Streichlinienauslenkung stromab relativ stark von der Aktuatoramplitude abhängt. Die Amplitude der höheren Harmonischen sind bezüglich der experimentellen Harmonischen allerdings zu klein.

\section{D.1.2 Begrenzung der zeitlichen Ableitung des Signals}

Die zeitliche Ableitung $\frac{d s(t)}{d t}$ des gesamten Signals nach dem Filter $\Delta s$ wird durch die Nichtlinearität begrenzt. Da die gemessene Auslenkung der Streichlinien für große Amplituden einem Dreiecksignal ähnelt, sollte man die zeitliche Ableitung des Signals in der Amplitude begrenzen bevor man das Signal wieder integriert. Dies hat den Nachteil, dass Integratoren sehr empfindlich auf Gleichanteile reagieren und der Gleichanteil durch Filterung wieder beseitigt werden muss.

Die Filterkaskade kann man nach den Methoden in Abschnitt 3.7.1 gewinnen, wenn man statt der dort auf die Streichlinienauslenkung bezogenen Sollübertragungsfunktion nun die auf die Ableitung der Auslenkung bezogene benutzt (also $i \omega H_{\text {soll }}$ ). Durch die Bildung der Ableitung 
werden die hohen Frequenzen zusätzlich stark angehoben. Sie werden bei der Filterkaskade jedoch schlechter übertragen, weil die n-fache Iteration von Filtern die hohen Frequenzen stark absenkt. Daher liefert dieses Verfahren nicht die gewünschten Ergebnisse. Es ist nicht möglich, sowohl die Signallaufzeit als auch das Amplitudenverhalten in Übereinstimmung mit den experimentellen Daten zu bringen. Wie in Abb. D.2 (unten) zu sehen, ist die Ausbreitungsgeschwindigkeit zu gering.

\section{D.1.3 Begrenzung des Signals}

Das gesamte Signal wird am Ausgang jedes Kaskadenelementes begrenzt. Dieses Modell kann die Signallaufzeiten der hohen Frequenzen gut abbilden und ist daher am geeignetsten zur Modellierung der globalen Instabilität. Die Harmonischen der Signale entsprechen jedoch nicht den im Experiment beobachteten. Die entstehende rechteckige Signalform kann aber durch die Tiefpasscharakteristik der Übertragungskette wieder geglättet werden. Da dieses Modell die Signallaufzeit über einen großen Frequenzbereich reproduzieren kann, wird es zur Modellierung der globalen Instabilität benutzt und für Regelungsversuche verwendet. 

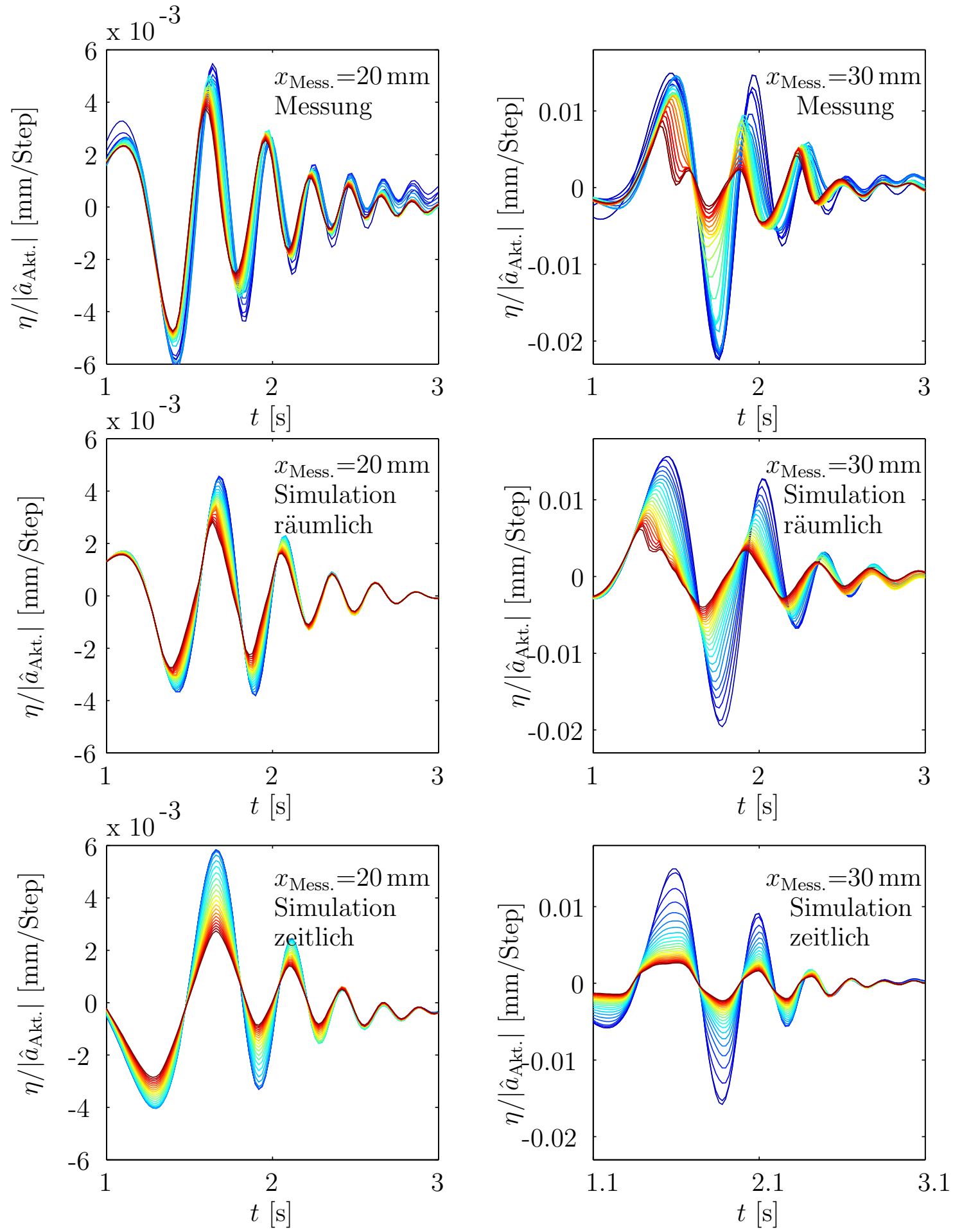

Abb. D.2: Vergleich von Filter-Kaskade und Experiment durch Sweepanregung. Räumlich: Begrenzung des Differenzsignals zwischen zwei Kaskaden-Elementen. Zeitlich: Begrenzung der Ableitung des Signals in jedem Kaskaden-Element. Farbe kodiert von blau nach rot die Aktuatoramplitude: blau $=50<\left|\hat{a}_{\text {Akt. }}\right|<1100=$ rot. 


\section{Anhang E}

\section{E.1 Modell des Strahl-Kanten-Systems auf die Streich- linienauslenkug bezogen}

Die üblicherweise in der Literatur betrachteten Größen Wechselgeschwindigkeit und Druckgradient sind hier nicht direkt zugänglich. Da die Streichlinie ein Integral über das Wechselfeld darstellt, kann nicht direkt auf das Wechselfeld zurückgeschlossen werden. In diesem Abschnitt wird daher die Zerlegung der Strahl-Kanten-Strömung aus dem Abschnitt 4.1 für die hier messbare Streichlinienauslenkung dargestellt.

\section{Erklärung der Abkürzungen und Symbole in Abbildung E.1}

$\hat{a}_{\mathrm{Akt}}^{\mathrm{ae}} \quad \quad \ddot{A q u i v a l e n t e}$ Aktuatorauslenkung in Steps, die gebraucht wird, um die gleiche Wirkung, wie die der Strahl-Kanten-Wechselwirkung hinsichtlich der Anregung der Instabilitätswelle, zu erzielen.

$\tilde{\eta}\left(x_{\mathrm{D}}\right) \quad$ gemessene Streichlinienauslenkung direkt unterhalb der Düse

$\tilde{\eta}_{\text {Inst }}\left(x_{\mathrm{D}}\right) \quad$ Streichlinienauslenkung aufgrund der Instabiliätswelle (nicht direkt messbar) direkt unterhalb der Düse

$\tilde{\eta}\left(x_{\text {Keil }}\right) \quad$ gemessene Streichlinienauslenkung direkt oberhalb der Keilposition; die gemessenene Streichlinienauslenkung wird mit der Streichlinienauslenkung aufgrund der Instabilitätswelle gleichgesetzt, der Einfluss des Nahfeldes des Keils wird dabei vernachlässigt.

Inst.Anreg $\quad A_{\text {Inst }}=\frac{\tilde{\eta}_{\text {Inst }}\left(x_{\mathrm{D}}\right)}{\hat{a}_{\text {Akt. }}^{\mathrm{e}} \mathrm{A}}$ Anregung der Instabilitätswelle durch den äquivalenten Aktuator

Aktuator $\quad A=\frac{\tilde{\eta}\left(x_{\mathrm{D}}\right)}{\hat{a}_{\text {akt. }}^{\mathrm{e}} \text {. }}$ Aktuatorübertragungsfunktion bzw. Störungsannahme des Freistrahls: Umsetzung der Aktuatorwechselauslenkung in die Wechselauslenkung der Streichlinie unterhalb der Düse 


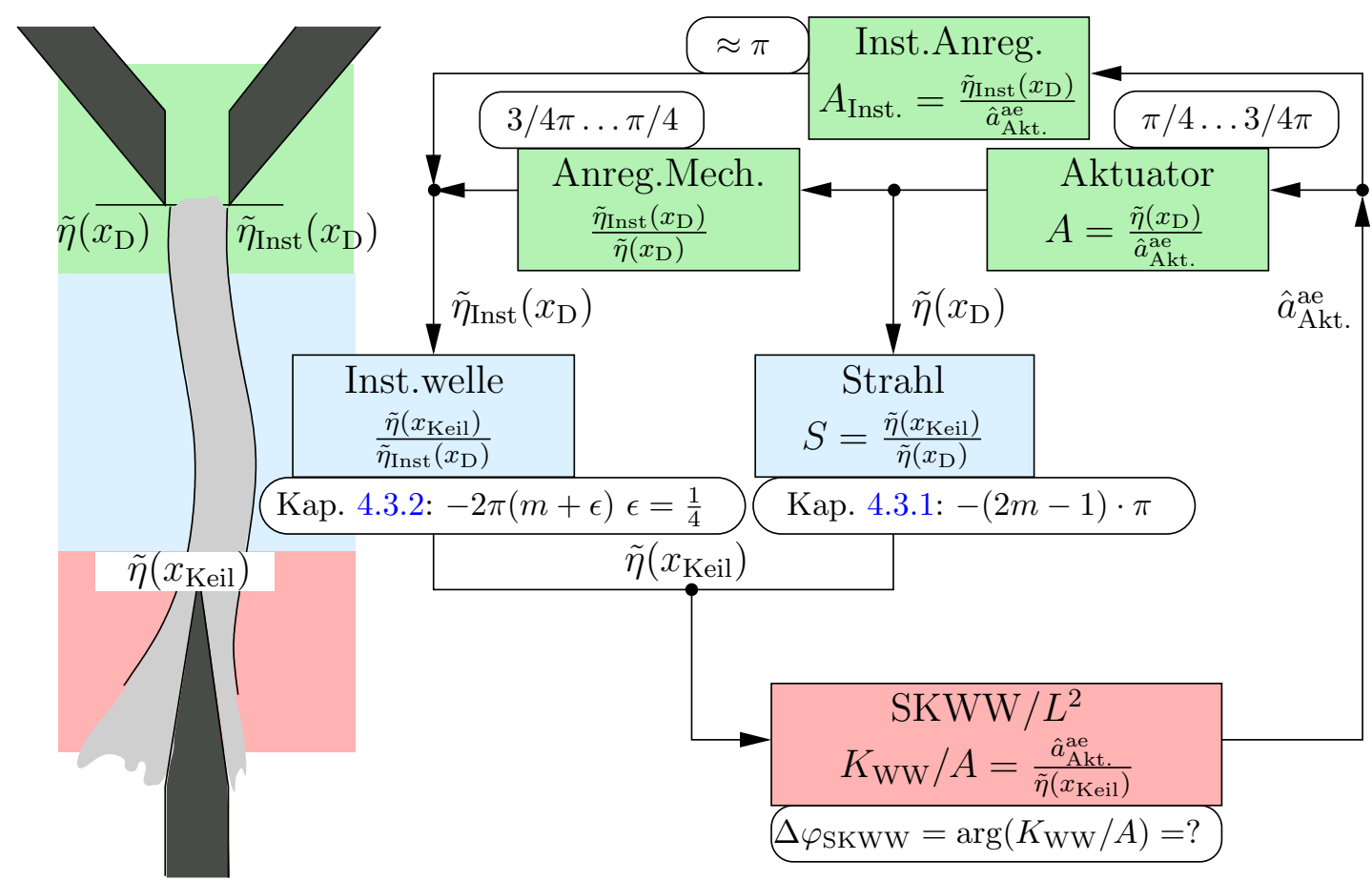

Abb. E.1: Zusammenstellung der Übertragungsfunktionen. In den abgerundeten Kästen sind die Phasen der Übertragungsfunktionen angegeben, dabei ist eine negative Phase eine Verzögerung.

Anreg.Mech $\quad \frac{\tilde{\eta}\left(x_{\mathrm{D}}\right)}{\hat{a}_{\text {Akt. }}^{\text {ae }}}$ Anregungsmechanismus der Instabilitätswelle durch das Feld in Düsennähe

Inst.welle $\quad \frac{\tilde{\eta}\left(x_{\text {Keil }}\right)}{\tilde{\eta}_{\text {Inst }}\left(x_{\mathrm{D}}\right)}$ Ausbreitung der Instabilitätswelle, ohne die Nahfelder an Düse und Keil, dazu stellt man sich die Instabilitätswelle im Nahfeld als eine aus dem Fernfeld zurückinterpolierte vor.

Strahl $\frac{\tilde{\eta}\left(x_{\text {Keil }}\right)}{\tilde{\eta}\left(x_{\mathrm{D}}\right)}$ Streichlinienauslenkung mit allen Nahfeldern an Düse und Keil $\mathrm{SKWW} / L^{2}$ Strahlkantenwechselwirkung: Die Wechselauslenkung am Keil führt zu einer äquivalenten Aktuatorauslenkung, auf die der gesamte physikalische Mechanismus der Strahl-Kanten-Wechselwirkung reduziert wird. $K_{\mathrm{WW}} / A=\frac{\hat{a}_{\text {akt. }}^{\text {ae }}}{\tilde{\eta}\left(x_{\text {Keil }}\right)}$ ist die Übertragungsfunktion, die diese Umsetzung beschreibt. Nach dem Modell des Abschnitts 4.1 ist die äquivalente Aktuatorauslenkung bezüglich $\tilde{v}_{\text {Inst }}\left(x_{\text {Keil }}\right)$ gegenphasig. Da nicht genau bekannt ist, an welcher $x$-Position die Wechselwirkung stattfindet und ob noch weitere Zeitkonstanten beteiligt sind, ist die Phasendrehung $\Delta \varphi_{\text {SKwW }}$ unbekannt. In Kap. 5 wird die Übertragungsfunktion $K_{\mathrm{WW}} / A$ näherungsweise bestimmt. 
Der Messung zugänglich sind nur die Streichlinienauslenkung im Bereich zwischen dem Düsenort $x_{\mathrm{D}}$ und dem Keilort $x_{\text {Keil }}$, sowie die Übertragungsfunktionen vom Aktuator zu den verschiedenen Messpositionen $x_{\text {Mess. }}$. Mit diesen Informationen soll nun die globale Instabilität so dargestellt werden, dass man den physikalischen Mechanismus der Strahl-Kanten-Wechselwirkung beschreiben kann. Dazu wird das Kraftfeld, das durch die Strahl-Kanten-Wechselwirkung erzeugt wird durch eine äquivalente Aktuatorauslenkung $\hat{a}_{\text {Akt. }}^{\text {ae }}$ beschrieben. Im Detail unterscheidet sich das Aktuatorfeld von dem Feld eines Dipols, der auf der Keilspitze - oder etwas stromab - sitzt. Im Nahfeld der Düse sollte der Unterschied aber sehr gering und damit die Anregung der Instabilitätswelle praktisch identisch sein.

\section{Phasenbeziehungen zwischen den Messgrößen}

Die Strouhalzahl stellt sich so ein, dass die Gesamtphasendrehung der relevanten Übertragungsfunktionen ein ganzzahliges Vielfaches von $2 \pi$ ergeben muss. Hier wird angenommen, dass die Ausbreitung der Information über den Strömungszustand an der Düse durch die Instabilitätswelle zum Keil, und dort durch die Wechselwirkung mit dem Keil und dem resultierenden Druckfeld zurück zur Düse übertragen wird, wie in Abb. 4.1 dargestellt. Die Phasenbeziehungen der in Abb. E.1 angegebenen Übertragungsfunktionen sind auf die Streichlinienauslenkung bezogen, so dass die eigentlich relevanten physikalischen Größen, nämlich die Wechselgeschwindigkeit und das Druckfeld der Strahl-Kanten-Wechselwirkung nur indirekt durch die sichtbare Streichlinienauslenkung beschrieben werden. Daher ist es notwendig, die Phasenbeziehung zwischen der Streichlinienauslenkung und dem Wechselgeschwindigkeitsfeld zu betrachten.

In Düsennähe kann das Wechselgeschwindigkeitsfeld näherungsweise aus der Streichlinienauslenkung $\tilde{\eta}(x)$ bestimmt werden, indem man die Streichlinie durch das Strömungsfeld $\underline{U}(x, y) \approx$ $\left[u(x, y), \tilde{v}\left(x_{\mathrm{D}}\right) e^{\sigma x}\right]$ unter Vernachlässigung von $\tilde{u}$ und mit der Annahme $u(x, y)=\bar{U}(x, y)=$ const. integriert. Eine Rechnung analog zu der in Abschnitt C.2 liefert dann

$$
\tilde{v}\left(x_{\mathrm{D}}\right)=\tilde{\eta}(x)(\sigma \bar{U}+i \omega) e^{-\sigma x} .
$$

Weiter stromab kann die Phasendifferenz zwischen $\tilde{\eta}(x)$ und $\tilde{v}(x):=\left.\tilde{v}(x, y)\right|_{y=0}$ mittels der WKB-Methode bestimmt werden. Nach Abb. 3.5 kann die Phasendifferenz in Abhängigkeit von dem Laufweg durch

$$
\arg \left(\frac{\tilde{v}(x)}{\tilde{\eta}(x)}\right)=\arg \left(\frac{\tilde{v}\left(x_{\mathrm{D}}\right)}{\tilde{\eta}\left(x_{\mathrm{D}}\right)}\right)-\int_{x_{\mathrm{D}}}^{x} \mathfrak{R e}\left\{i \nabla_{x} \ln \left(\tilde{v} / v_{\text {Akt. }}^{\mathrm{n}}\right)\right\}-\mathfrak{R e}\left\{i \nabla_{x} \ln \left(\tilde{\eta} / \zeta_{\text {Akt. }}^{\mathrm{n}} .\right)\right\} d x(\mathrm{E} .2)
$$

berechnet werden. Der Realteil des komplexen Phasengradienten ist im Gegensatz zur absoluten Phase von $\tilde{v}(x, y)$ näherungsweise unabhängig von der $y$-Position. Für $2 \mathrm{~Hz}, U_{\mathrm{D}}=$ $5.36 \mathrm{~cm} / \mathrm{s}$ ergibt sich aus Gleichung (E.1) $\arg \left(\tilde{v}\left(x_{\mathrm{D}}\right) / \tilde{\eta}\left(x_{\mathrm{D}}\right)\right) \approx 0.4 \pi$, mit $\sigma=1 /(2 d)$. Da $\mathfrak{R e}\left\{i \nabla_{x} \ln \left(\tilde{v} / v_{\text {Akt. }}^{\mathrm{n}}\right)\right\}>\mathfrak{R e}\left\{i \nabla_{x} \ln \left(\tilde{\eta} / \zeta_{\text {Akt. }}^{\mathrm{n}}\right)\right\}$, nimmt die Phasendifferenz mit dem Laufweg ab und sinkt auf $\left.\arg \left(\left.\tilde{v}(x, y)\right|_{y=0} / \tilde{\eta}(x)\right)\right|_{x=40 \mathrm{~mm}} \approx 0.2 \pi$, wenn man Gl. (E.2) auswertet. Allerdings 
kann durch die Veränderung des Phasengradienten mit steigender Auslenkungsamplitude dieser Unterschied noch größer werden. Eine Abschätzung aus dem sehr vereinfachenden Modell des Abschnitts B.1.2 und Abb. 3.9 zeigt, dass der Phasengradient von $\tilde{v}$ um bis zu etwa $5 \%$ bei großen Auslenkungen ansteigen kann. Das inverse Problem, die Phase von $\tilde{v}(x)$ aus der Phase von $\tilde{\eta}(x)$ zu berechnen, ist daher für größere Düsenabstände und Auslenkungsamplituden nicht einfach zu lösen.

Die bisher bekannten Phasenbeziehung der einzelnen Übertragungsfunktionen sind in Abb. E.1 eingetragen. Zusammenfassend ergibt sich also:

Strahlrezeptivität: Aus Abschnitt C.2 ergibt sich für die Übertragungsfunktion $A=\frac{\tilde{\eta}\left(x_{\mathrm{D}}\right)}{\hat{a}_{\text {Akt. }}^{\mathrm{e}} \text {. }}$ für die hier vorkommenden $\mathrm{Sr}_{\mathrm{d}}$ eine vorlaufende Phase zwischen $3 / 4 \pi$ und $\pi / 4$.

Anregung der Instabilitätswelle: Aus Abschnitt C.3 ergibt sich für die Übertragungsfunktion $\frac{\tilde{\eta}_{\text {Inst }}\left(x_{\mathrm{D}}\right)}{\hat{a}_{\mathrm{Akt}}^{\mathrm{e}} \mathrm{e}}$ eine Phase von etwa $\pi$.

Strahl-Kanten-Wechselwirkung: Die Phase der Strahl-Kanten-Wechselwirkung ist aufgrund der unbekannten Position des effektiven Orts der Wechselwirkung zwischen Strahl und Keil nicht bekannt. Die Phase zwischen dem Wechselgeschwindigkeitsfeld und der äquivalenten Aktuatorauslenkung $\hat{a}_{\text {Akt. }}^{\text {ae }}$ ist näherungsweise durch die Beziehung

$$
\hat{a}_{\text {Akt. }}^{\mathrm{ae}} \propto\left(i \omega^{\prime}\right)^{-2} \cdot \tilde{v}_{\mathrm{Inst}}^{\mathrm{eff}} \quad \text { mit } \omega^{\prime}=\text { Dopplerverschobener Frequenz }
$$

gegeben, wobei $\tilde{v}_{\text {Inst }}^{\text {eff }}$ die Schnelle am effektiven Ort der Wechselwirkung symbolisiert. Die Streichlinienintegration zeigt, dass das Wechselgeschwindigkeitsfeld und die Streichlinienauslenkung eine schwer zu beschreibende Phasenverschiebung haben. Die Gesamtphasendifferenz bezogen auf den Aktuator, ist durch Gl. (E.2) gegeben und nimmt dann mit dem Laufweg leicht ab. Die nach dem obigen Schema berechneten Phasen von $\tilde{v} / \tilde{\eta}$ für $x=10 d, U_{\mathrm{D}}=5.36 \mathrm{~cm} / \mathrm{s}$ ergeben $\left.\arg \{\tilde{\eta} / \tilde{v}\}\right|_{2 \mathrm{~Hz}} \approx 0.2 \cdot(2 \pi),\left.\arg \{\tilde{\eta} / \tilde{v}\}\right|_{1 \mathrm{~Hz}} \approx-0.1 \cdot(2 \pi)$ und $\left.\arg \{\tilde{\eta} / \tilde{v}\}\right|_{0.6 \mathrm{~Hz}} \approx-0.15 \cdot(2 \pi)$. Verallgemeinert man dies, so ergibt sich für die Phasenbeziehung zwischen der Streichlinienauslenkung am Ort der effektiven Wechselwirkung und der Auslenkung des hypothetischen Aktuators

$$
\arg \left\{\hat{a}_{\text {Akt. }}^{\text {ae }} / \tilde{\eta}_{\text {Inst }}^{\text {eff. }}\right\} \approx(-0.8 \cdots-1.2) \pi
$$

Hinzu kommt eine Phasendrehung die von Kaykayoglu \& Rockwell ([24, 25]) mit $\approx-\pi / 2$ angeben wird und die im wesentlichen durch die Ausbreitung der Störung vom Ort $x_{\text {Keil }}$ zum Ort der effektiven Wechselwirkung gegeben ist. Die sich ausbreitende Störung muss dabei die Randbedingungen am Keil erfüllen und ist daher nicht die Strahlinstabilität. Damit ergibt sich als Schätzung für die Gesamtphasendrehung der Übertragungsfunktion $K_{\mathrm{WW}} / A$ der Strahl-Kanten-Wechselwirkung:

$$
\Delta \varphi_{\mathrm{SKWW}}=(-0.8 \cdots-1.2) \pi-\pi / 2 .
$$




\section{Anhang F}

\section{F.1 Analytisches Signal durch Karhunen-Loève-Trans- formation (KLT) der Streichlinieauslenkung}

In Kap. 4.7.1 wurde nur die Streichlinienauslenkung an einer Messposition zur Bildung des analytischen Signals benutzt. Aufgrund der hohen räumlichen Korrelationen und des Wellencharakters der Instabilitätswellenausbreitung wurde davon ausgegangen, dass dies die Systemdynamik ausreichend beschreibt. Mittels einer auf die Wellenausbreitung erweiterten KarhunenLoève-Transformation (KLT) kann diese Annahme bestätigt werden und zusätzlich die gesamte zur Verfügung stehende räumliche Information über den Systemzustand genutzt werden. Die Systemdynamik kann durch die KLT auf wenige komplexe Projektionskoeffizienten reduziert werden, deren Dynamik sich in Bezug auf die Synchronisation kaum von der Betrachtung des analytischen Signals unterscheidet.

Die KLT (auch Proper Orthogonal Decomposition (POD) genannt) dient dazu, eine optimale orthogonale Basis von Zeitreihen räumlicher Felder („Schnappschüsse“ genannt) zu berechenen. Diese Basis ist optimal im Sinne aller linearen Zerlegungen der Zeitreihe: Zu einer bestimmten Anzahl von Eigenfunktionen der berechneten Basis wird, durch die Projektion der Zeitreihe auf diese Basis, die maximale mittlere Leistung aus der Zeitreihe entnommen. Die Projektionskoeffizienten bilden die Dynamik des Systems ab und charakterisieren so den Systemzustand zu einer bestimmten Zeit. Dieser kann zur Analyse des Systems genutzt werden und allgemeiner auch zur niederdimensionalen Modellierung und Regelung von dynamischen Systemen ([12][32]).

Da die räumliche Struktur der physikalischen Moden durch die Dispersionsrelation an die Frequenz gekoppelt ist, kann durch die KLT die Momentanfrequenz der verschiedenen Moden analysiert werden. Die KLT-Eigenvektoren fallen nicht zwangsläufig mit den physikalischen Moden zusammen. Dies kann allerdings annähernd durch geeignete Normierung der Streichlinienauslenkung:

$$
\underline{\eta}(t)=\left[\eta\left(x_{1}, t\right), \ldots, \eta\left(x_{\mathrm{n}}, t\right)\right]^{t}
$$

erreicht werden. Mittels der KLT kann die Streichlinienbewegung durch wenige zeitabhängige 
Koeffizienten beschrieben werden, die durch Projektion von $\underline{\eta}(t)$ zu einer festen Zeit auf die Eigenvektoren $\underline{E}$ gewonnen werden.

Zur Berechnung der Eigenvektoren wird das mittelwertfreie $\eta\left(x_{\mathrm{i}}, t\right)$ auf den über die Zeit gemittelten rms-Wert am Ort $x_{\mathrm{i}}$ normiert

$$
\eta^{0}\left(x_{\mathrm{i}}, t\right)=\frac{\left(\eta\left(x_{\mathrm{i}}, t\right)-\left\langle\eta\left(x_{\mathrm{i}}, t\right)\right\rangle_{\mathrm{t}}\right)}{\operatorname{rms}\left(\eta\left(x_{\mathrm{i}}, t\right)-\left\langle\eta\left(x_{\mathrm{i}}, t\right)\right\rangle_{\mathrm{t}}\right)} .
$$

Die KLT liefert sukzessive eine Basis von Eigenvektoren $\underline{E}_{\mathrm{i}}$, indem aus dem Restsignal $\underline{\eta}_{\mathrm{i}}^{0}=$ $\underline{\eta}^{0}-\sum_{k=0}^{i-1}\left(\underline{\mathrm{E}}_{\mathrm{k}}^{\mathrm{t}} \cdot \underline{\eta}^{0}\right) \underline{\eta}^{0}$ der Eigenvektor bestimmt wird, der die größte mittlere Leistung der Projektion auf den Eigenvektor bezogen auf die Norm des Eigenvektors liefert:

$$
\max _{\underline{E}} \frac{\left.\left.\langle| \underline{\mathrm{E}}^{\mathrm{t}} \cdot \underline{\eta}_{\mathrm{i}}^{0}(\mathrm{t})\right)\left.\right|^{2}\right\rangle_{\mathrm{t}}}{\underline{\mathrm{E}}^{\mathrm{t}} \cdot \underline{\mathrm{E}}}=\frac{\left\langle\left|\underline{E}_{\mathrm{i}} \cdot \underline{\eta}_{\mathrm{i}}^{0}(t)\right|^{2}\right\rangle_{\mathrm{t}}}{\underline{\mathrm{E}}_{\mathrm{i}}^{\mathrm{t}} \cdot \underline{\mathrm{E}}_{\mathrm{i}}} .
$$

Ohne die obige Normierung (Gl. (F.2)) der Zeitreihen auf den jeweiligen rms-Wert am Ort $x$ würden hauptsächlich die großen Amplituden düsenfernerer Orte die Zerlegung bestimmen und der gewünschte Wellencharakter der KLT-Eigenvekoren würde verloren gehen.

Es kann gezeigt werden, dass das obige Orthogonalisierungsverfahren auch durch Diagonalisierung der Korrelationsmatrix

$$
R=\left\langle\underline{\eta}^{0} \underline{\eta}^{0}\right\rangle_{\mathrm{t}}
$$

gewonnen werden kann:

$$
R \underline{E}_{\mathrm{i}}=\lambda_{\mathrm{i}} \underline{E}_{\mathrm{i}},\left|\underline{E}_{\mathrm{i}}\right|=1 .
$$

Damit erhält man ein System von $n$ Basisvektoren $\left\{\underline{E}_{\mathrm{i}}\right\}_{i=1}^{n}$. Die Eigenwerte $\lambda_{\mathrm{i}}$ können als mittlere Leistung, die aus dem Signal durch die Projektion auf $\underline{E}_{\mathrm{i}}$ extrahiert wird, interpretiert werden. Ordnet man die $\underline{E}_{\mathrm{i}}$ nach der Größe von $\lambda_{\mathrm{i}}$ an, so braucht man nur wenige Eigenvektoren $\left\{\underline{E}_{\mathrm{i}}\right\}_{i=1}^{l}, l<n$ um das Streichlinienauslenkungssignal aus den Projektionskoeffizienten $p_{\mathrm{i}}$ annähernd zusammenzusetzen:

$$
\underline{\eta}^{0}(t) \approx \sum_{i=0}^{l} p_{\mathrm{i}}(t) \underline{E}_{\mathrm{i}} \text { mit } p_{\mathrm{i}}(t)=\underline{\mathrm{E}}_{\mathrm{i}}^{\mathrm{t}} \cdot \underline{\eta}^{0}(\mathrm{t}) .
$$

In Kap. 4.7.1 soll der Systemzustand II auf diese Weise untersucht werden. Die räumlichen Basisfunktionen werden für den hochfrequenten und den niederfrequenten Anteil der Zeitreihe getrennt bestimmt, damit erreicht man in der Projektion der Streichlinienauslenkung auf diese Basis eine Trennung von Mode I und Mode II.

Die Eigenvektoren, die man aus $\underline{\eta}^{0}(t)$, bzw. aus $\underline{\eta}^{0 \mathrm{TP}}$ und $\underline{\eta}^{0^{\mathrm{HP}}}$ erhält (siehe Gl. (4.14)), lassen sich aufgrund ihrer etwa um $\pi / 2$ verschobenen räumlichen Phase zu entsprechenden komplexen Eigenvektoren

$$
{\underline{E_{\mathcal{k}}}}_{\mathrm{k}}=\sqrt{\lambda_{2 \mathrm{k}-1}} \underline{E}_{2 \mathrm{k}-1} \pm i \sqrt{\lambda_{2 \mathrm{k}}} \underline{E}_{2 \mathrm{k}}
$$




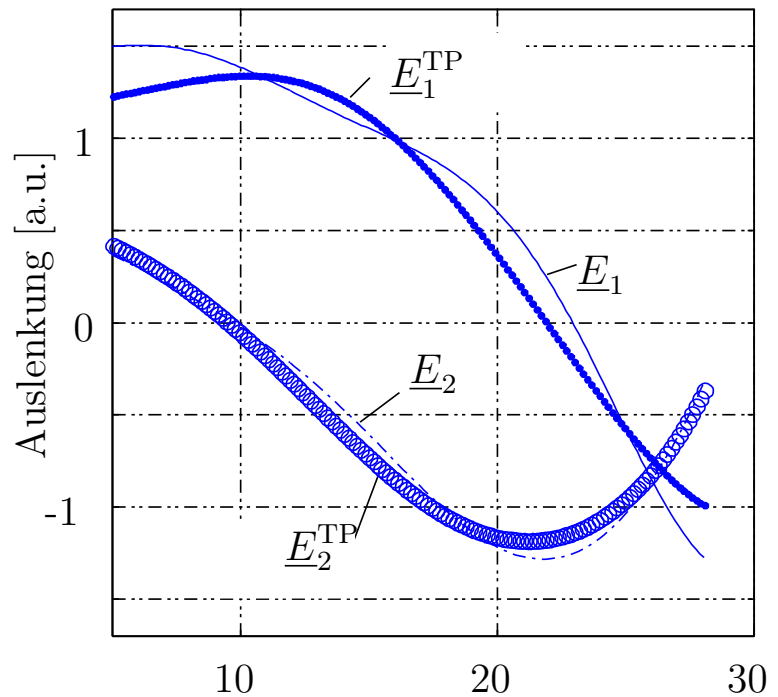

Düsenabstand $\mathrm{x}[\mathrm{mm}]$

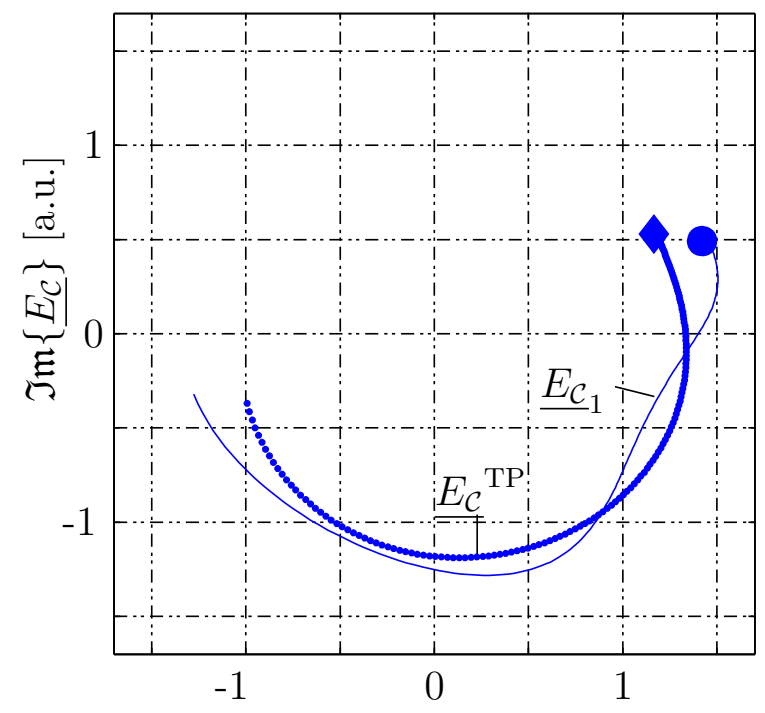

$\mathfrak{R e}\left\{\underline{E_{\mathcal{C}}}\right\}$
Düsenabstand $\mathrm{x}[\mathrm{mm}]$

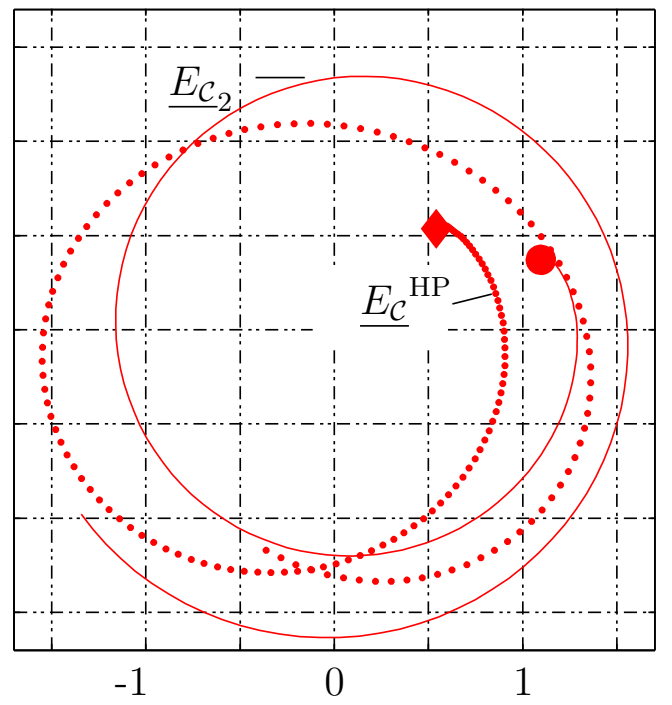

$\mathfrak{R e}\left\{\underline{E_{\mathcal{C}}}\right\}$

Abb. F.1: Eigenvektoren der KLT der vollständigen Zeitreihen und der nach Tiefpassanteil und Hochpassanteil getrennten Zeitreihen. Oben: Eigenvektoren als Funktion von $x$. Unten: die daraus zusammengestellten komplexen Eigenvektoren (Parameter $x$, Markierungen stehen für $\left.x=x_{\mathrm{D}}\right)$. 
zusammenfassen, wie in Abb. F.1 gezeigt. Mit den so angeordneten $\underline{E}$ kann (näherungsweise) eine Wellenausbreitung beschrieben werden. Die komplexen Eigenvektoren werden in Gl. (F.7) so zusammengesetzt, dass sie eine stromab laufende Welle entsprechend $(d / d x) \arg \left(\underline{E_{\mathcal{C}}}\right)<0$ darstellen.

Die räumliche Struktur (Abb. F.1) zeigt erwartungsgemäß etwa eine Verdreifachung der räumlichen Phasendrehung von Mode II bezüglich Mode I. Die größten qualitativen Unterschiede findet man zwischen dem Eigenvektor der tieffrequenten Komponente der Zeitreihe ${\underline{E_{\mathcal{C}}}}^{\mathrm{TP}}$ und dem Eigenvektor der gesamten Zeitreihe ${\underline{E_{\mathcal{C}}}}_{1}$.

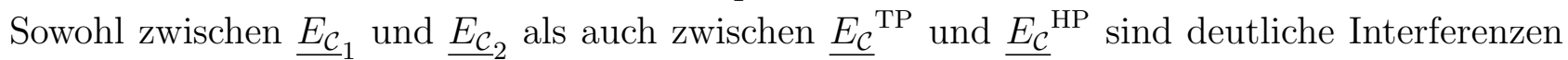
zu erkennen. Man könnte daher versucht sein, diese als Hinweis auf eine physikalische Kopplung zwischen den Moden zu interpretieren. Dabei ist aber zu bedenken, dass auch zwei völlig entkoppelte, sich mit verschiedenen Wellenzahlen und Frequenzen ausbreitende Wellen durch die KLT nicht wieder in die ursprünglichen, einzelnen Wellen zerlegt werden.

Um $\eta^{0}$ des Systemzustands II anzunähern, genügt für jeden Frequenzbereich jeweils ein komplexer Projektionskoeffizient, da das Eigenwertspektrum von $\underline{\eta}^{0^{\mathrm{TP}}}$ und $\underline{\eta}^{0^{\mathrm{HP}}}$ nach den ersten beiden Eigenwerten sehr schnell abnimmt. Es ist nicht zu erwarten, dass ${\underline{E_{\mathcal{C}}}}^{\text {TP }}$ orthogonal zu ${\underline{E_{\mathcal{C}}}}^{\mathrm{HP}}$ ist, so dass die Kreuzprojektionen $\left|{\underline{E_{\mathcal{C}}}}^{\mathrm{HP}}{ }^{t} \underline{\eta}^{0^{\mathrm{TP}}}\right|,\left|{\underline{E_{\mathcal{C}}}}^{\mathrm{TP}{ }^{t}} \underline{\eta}^{{ }^{\mathrm{HP}}}\right|$ nicht verschwinden.

Die Projektionskoeffizienten $p_{\mathcal{C}}$ können nun als analytisches Signal genutzt werden. In Abb. 4.16 sind die $p_{\mathcal{C}}$ und das analytische Signal der Zeitreihe $\eta_{\mathcal{C}}$ an einem Messort verglichen. Die Unterschiede sind sehr gering. 


\section{Literaturverzeichnis}

[1] Bamberger A., Bänsch E., Siebert, K.: Experimental and numerical investigation of edge tones Z. Angew. Math. Mech. 84, No. 9, 632 - 646 (2004) 2

[2] Betchow, Robert; Criminale, William O. Jr.:Stability of Parallel Flows,Academic Press, London and New York (1967) 22

[3] Billoud, G.; Galland, M. A.; Huu, C. H.; Candel, S.: Adaptive active control of instabilities in C. A. Rogers und C. R. Fuller (Hrsg.) Proceedings of the 1st Conference on Recent Advances in Active Control of Sound and Vibration, Blacksbury, VA, April 15-17, 95-107 (1991) 4

[4] Brackenridge J.B.: Transverse oscillations of a liquid jet JASA 32, 1237 (1960) 2

[5] Brown G.B.: The vortex motion causing edge tones Proc. Phys. Soc. 49, 493 (1937) 2

[6] Chandrasekhar, S.: Hydrodynamic and Hydromagnetic Stability,Oxford University Press, London and New York (1961) 22

[7] Chomaz J. M.; Huerre P.; Redekopp L. G.: Bifurcations to Local and Global Modes in Spatially Developing Flows Phys. Rev. Lett. 60, 25-28 (1988) 47

[8] Crighton, D. G.: The jet edge-tone feedback cycle; linear theory for the operating stages, J. Fluid Mech. 234, 361-391 (1992) 2, 48, 53, 55

[9] Dierken, C.: Rezeptivität einer ablösenden Strömungsscherschicht bei hohen Frequenzen, Diplomarbeit am 3. Phys. Inst. der Univ. Göttingen (1995) 6

[10] Drazin, P.G.; Reid, W.H.: Hydrodynamic stability,Cambridge University Press (1981)

[11] Driscoll, T.: A MATLAB Toolbox for Schwarz-Christoffel Mapping, ACM Transactions on Mathematical Software 6 (1996) 132

[12] Fitzsimons, P. and Rui, C.: Determining low-dimensional models of distributed systems in Advances in Robust Nonlinear Control Systems, ASME DSC 53,9-15 (1993) 151 
[13] Ffowcs Williams, J. E.; Huang, X. Y.: Active stabilization of compressor surge, J. Fluid Mech. 204, 245-262 (1989) 3

[14] Fujisawa, N., Takizawa, Y., Kohno T., Tomimatsu S.: Active control of flow oscillations in jet-wedge system by acoustic feedback Journal of Fluids and Structures 19, 111-122 (2004) 3

[15] Fujisawa, N., Takizawa, Y.: Study of feedback control of edge tone by simultaneous flow visualization, control and PIV measurement Meas. Sci. Technol. 14, 1412-1419 (2003) 3

[16] Gaster M., Kit E., Wygnanski I.: Large-scale structures in a forced turbulent mixing layer J. Fluid Mechanics, 150, 23-39 (1985) 24

[17] Gautschy A., Glatzel W.: A new method to solve linear pulsation problems Mon. Not. Roy. Astro. Soc., 245, 154-60 (1990) 22

[18] Godrèche, C.; Manneville, P.: Hydrodynamics and Nonlinear Instabilities,Cambridge University Press (1998) 24

[19] Holger, D. K.; Wilson, T. A.; Beavers, G. S.: Fluid mechanics of the edgetone, J. Acoust. Soc. Am. 62, 1116-1128 (1977) 2

[20] Howe, M.S.: Edge, cavity and aperture tones at very low Mach numbers. J. Fluid Mech. 330, 61-84 (1997) 53, 55

[21] Howell, L. H.; Trefethen, L. N.: A modified Schwarz-Christoffel transformation for elongated regions, SIAM J. Sci. Stat. Comput. 11, 928-949 (1990) 132

[22] Huerre P., Scott J.F.: effects of critical layer structure on the non-linear evolution of waves in free shear layers Proceedings of the royal society of london series A, 371 1747, 509-524 (1980) 123

[23] Karamcheti, K.; Bauer, A. B.; Shields, W. L.; Stegen, G. R.; Wooley, J. P.: Some Features of an Edge-Tone Flow Field, NASA SP-207, 275-304 (1969) 46

[24] Kaykayoglu, R.; Rockwell, D.: Unstable jet-edge interaction. Part 1. Instantaneous pressure fields at a single frequency, J. Fluid Mech. 169, 125-149 (1986) 48, 150

[25] Kaykayoglu, R.; Rockwell, D.: Unstable jet-edge interaction. Part 2: Multiple frequency pressure fields, J. Fluid Mech. 169, 151-172 (1986) 48, 150

[26] Lucas, M.; Rockwell, D.: Self-excited jet: upstream modulation and multiple frequencies, J. Fluid Mech. 147, 333-352 (1984) 2 
[27] Maurel, A.; Ern, P.; Zielinska, B. J. A.; Wesfreid, J. E.: Experimental Study of SelfSustained Oscillations in a Confined Jet, Phys. Rev. E 54 (4), 3643-3651 (1996) 31, 53

[28] Maurel A., Pagneux V., Wesfreid J.E.: Mean Flow Correction as Non Linear Saturation Mechanism in Instabilities Europhys. Letters, 32 3, 217-222 (1995) 123

[29] Möser, M.: Aktive Kontrolle einfacher, selbsterregter Resonatoren, Acustica 69, 175-184 (1989) 3

[30] Nyborg W.L. et al.:Acoustical characteristics of the jet-edge and jet-edge-resonator systems JASA 52, 293 (1952). 2

[31] Kuo, S., M.; Morgan, D. R.: Active Noise Control Systems, Wiley, New York (1996) 4

[32] Ma, X., Azeez, M. A. F., and Vakakis, A. F.: Nonlinear normal modes and nonparametric system identification of nonlinear oscillators Mechanical Systems and Signal Processing 14(1), 37-48 (2000) 151

[33] Michalke, A.: On spatially growing disturbances in inviscid shear layer,Journal Fluid Mechanics,(19),521-44 (1965) 22

[34] Ohring, S.: Calculations of self-excited impinging jet flow, J. Fluid Mech. 163, $69-98$ (1986) 48

[35] Ott, E.; Grebogi, C.; Yorke, J. A.: Controlling Chaos, Phys. Rev. Lett. 64 (11), 1196-1199 (1990) 4

[36] Pikovsky A., Rosenblum, M., Kurths, J.: Synchronization. A universal concept in nonlinear sciences, Cambridge University Press (2001) 67

[37] Powell, A.: On Edge Tones and Associated Phenomena, Acustica 3, 233-243 (1953) 46, 53

[38] Powell, A.: On the Edgetone, J. Acoust. Soc. Am. 33 (4), 395-409 (1961) 1, 2, 46

[39] Preckel, H.: Dynamik und Steuerung der Strahl-Kanten-Strömung, Diss. Math.-Nat. Fak. Univ. Göttingen (1999) 3, 5, 24, 132, 137

[40] Raghu, S., Monkewitz P.: The bifurcation of a hot round jet to limit-cycle oscillations Physics of Fluids A 3, 501-503 (1991) 57

[41] Rayleigh, Lord: On the stability, or instability, of certain fluid motions,Proc. London Math. Soc.,(11),57-70 (1880) 22

[42] Roussopoulos, K.: Feedback control of vortex shedding at low Reynolds numbers, J. Fluid Mech. 248, 267-296 (1993) 3 
[43] Sondhaus, C.: Über die beim Ausströmen der Luft entstehenden Töne, Ann. Phys. (Leipzig) 91, $126 \mathrm{ff}$ und 214ff (1854) 2, 45

[44] Vaudrey, M.A.: Adaptive control methods for non-linear self-excited systems Dissertation Polytechnic Institute and Stateuniversity Blacksburg, Virginia (2001) 3 


\section{Dank}

Herrn Prof. Dr. Ronneberger danke ich für die nette freundschaftliche Atmosphäre während der Zeit am Dritten Physikalischen Institut. Die intensiven Diskussionen mit ihm und seine Bereitschaft, allen Fragen bis auf den Grund nachzugehen, haben mir sehr geholfen.

Bei Herrn Prof. Dr. Parlitz möchte ich mich für die Übernahme des Korreferats bedanken. Vielen Dank auch Herrn Geiling für seine Arbeit an der Schrittmotorkarte.

Dorothee Ickler und Matthias Jüschke danke ich für das Korrekturlesen der Arbeit.

Allen jetzigen und ehemaligen Arbeitsgruppenmitgliedern, insbesondere Holger Opfer, Michael Hartmann und Hiro Tokuno, möchte ich für die nette Arbeitsatmosphäre danken.

Danken möchte ich allen, die mich während der Zeit der Promotion unterstützt haben, besonders meiner Frau und meinen Eltern. 



\section{Lebenslauf}

\begin{tabular}{|c|c|}
\hline Name: & Arno Ickler \\
\hline geboren: & 03.April 1972 in Bad Hersfeld \\
\hline Staatsangehörig- & deutsch \\
\hline \multicolumn{2}{|l|}{ keit: } \\
\hline Familienstand: & verheiratet, 2 Kinder \\
\hline $1978-1982$ & Grundschule Obergeis \\
\hline $1982-1989$ & Gesamtschule Geistal in Bad Hersfeld \\
\hline $1989-1991$ & Modellschule Obersberg in Bad Hersfeld \\
\hline Juni 1991 & Abitur \\
\hline $1991-1992$ & $\begin{array}{l}\text { Mathematik- und Physikstudium an der Georg-August-Universität in } \\
\text { Göttingen }\end{array}$ \\
\hline $1992-1993$ & Zivildienst beim Deutschen Roten Kreuz in Bad Hersfeld \\
\hline $1993-1995$ & $\begin{array}{l}\text { Mathematik- und Physikstudium an der Georg-August-Universität in } \\
\text { Göttingen }\end{array}$ \\
\hline $10 / 1995-10 / 1997$ & $\begin{array}{l}\text { Diplomarbeit am Dritten Physikalischen Institut der Universität } \\
\text { Göttingen unter Anleitung von Prof. Dr. D. Ronneberger. Titel der Ar- } \\
\text { beit: „Analyse von Turbulenzspektren der gestörten Kanalströmung“ }\end{array}$ \\
\hline Oktober 1997 & Diplomprüfung \\
\hline Juni 1998 & 1.Staatsexamen Lehramt an Gymnasien: Mathematik und Physik \\
\hline 07/1998-09/1999 & $\begin{array}{l}\text { Anstellung am Vierten Physikalischen Institut als wissenschaftliche } \\
\text { Hilfskraft }\end{array}$ \\
\hline 10/1998-12/1998 & $\begin{array}{l}\text { zusätzlich Anstellung am Dritten Physikalischen Institut als wissen- } \\
\text { schaftliche Hilfskraft zur Betreuung des Fortgeschrittenen Praktikums }\end{array}$ \\
\hline 02/1999-09/1999 & $\begin{array}{l}\text { Stipendiat des Graduiertenkollegs „Strömungsinstabilitäten und Tur- } \\
\text { bulenz“ im Projekt „Rückgekoppelte und rückkopplungsfreie Steue- } \\
\text { rung instabiler Scherströmungen“ und Beginn der vorliegenden Dis- } \\
\text { sertation bei Prof. Dr. Ronneberger }\end{array}$ \\
\hline seit Oktober 1999 & $\begin{array}{l}\text { Anstellung am Dritten Physikalischen Institut als wissenschaftlicher } \\
\text { Mitarbeiter zur Betreuung des Fortgeschrittenen Praktikums }\end{array}$ \\
\hline 10/1999-03/2004 & zusätzlich assoziiertes Mitglied des Graduiertenkollegs \\
\hline
\end{tabular}

